\title{
The Role of CHD1 during Mesenchymal Stem Cell Differentiation
}

\author{
Dissertation \\ for the award of the degree \\ "Doctor rerum naturalium (Dr. rer. nat.)" \\ Division of Mathematics and Natural Sciences \\ of the Georg-August-Universität Göttingen
}

\author{
submitted by \\ Simon Baumgart \\ born in Gießen
}

Göttingen, 2015 
Members of the Thesis Committee:

Prof. Dr. Steven A. Johnsen (Reviewer)

Department of General, Visceral and Pediatric Surgery

University of Göttingen Medical School, Göttingen

Prof. Dr. Heidi Hahn (Reviewer)

Department of Human Genetics

University of Göttingen Medical School, Göttingen

Prof. Dr. Jürgen Wienands

Department of Cellular and Molecular Immunology University of Göttingen Medical School, Göttingen

Date of oral examination: $22^{\text {nd }}$ of February, 2016 


\section{Affidavit}

I hereby declare that the PhD thesis entitled "The Role of CHD1 during Mesenchymal Stem Cell Differentiation" has been written independently and with no other sources and aids than quoted.

Simon Baumgart

December, 2015

Göttingen 


\section{Table of Contents}

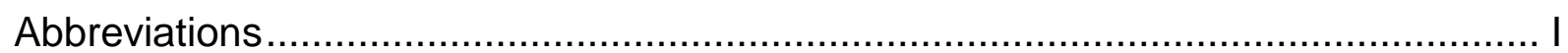

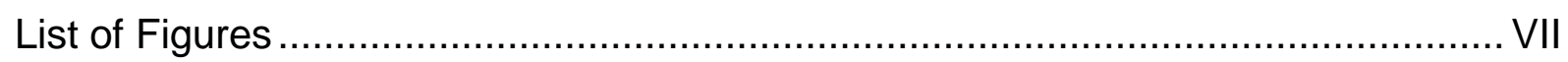

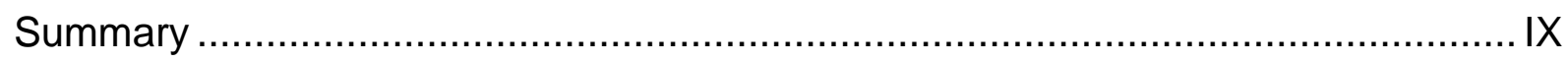

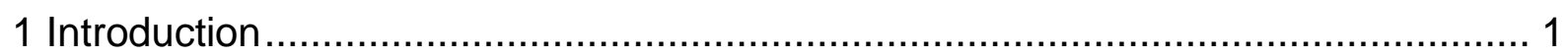

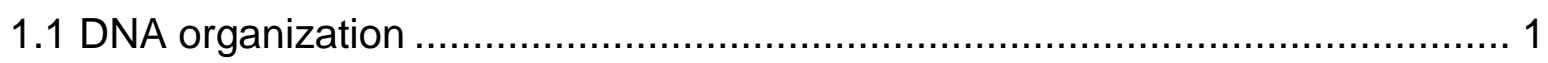

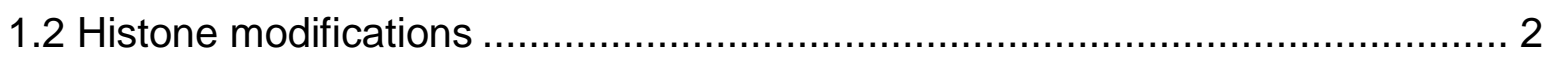

1.3 Deciphering the "histone code"................................................................. 3

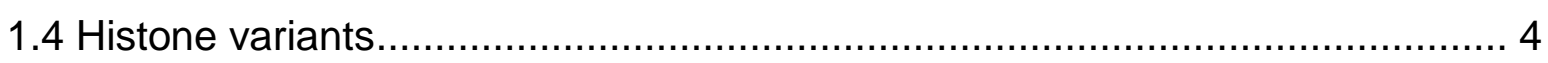

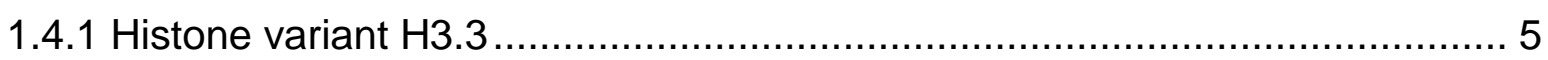

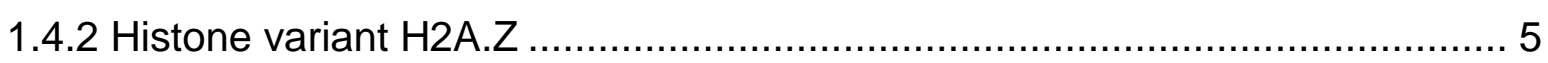

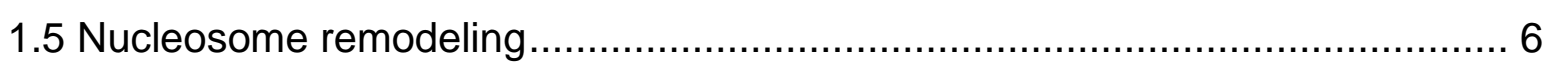

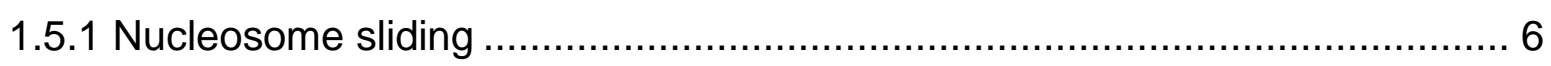

1.5.2 Ejection and histone replacement/removal ........................................... 9

1.5.3.1 Histone dynamics in transcription ........................................................ 9

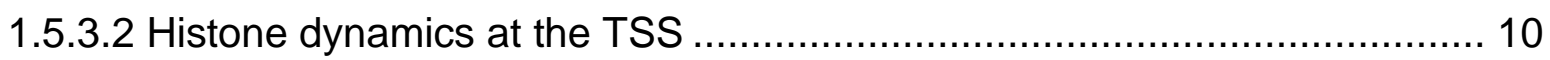

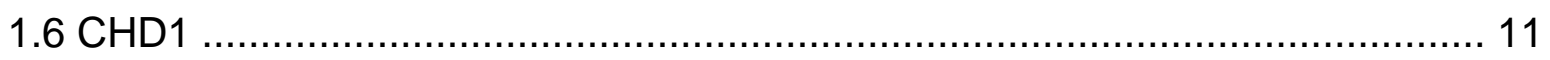

1.6.1 The role of CHD1 in yeast and drosophila ................................................ 11

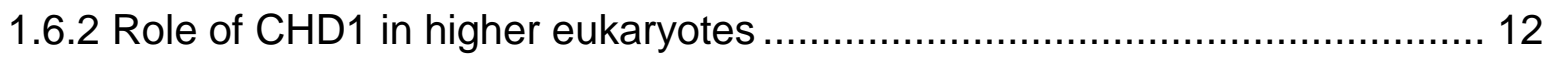

1.7 Stem cells and differentiation .............................................................. 14

$1.8 \mathrm{MSC}$ and their differentiation potential ................................................... 16

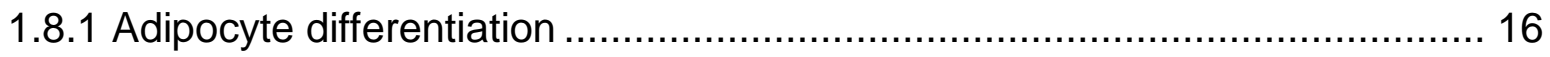

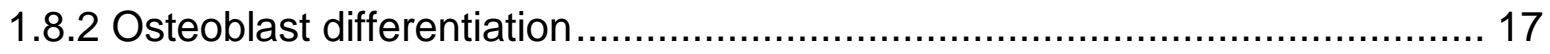

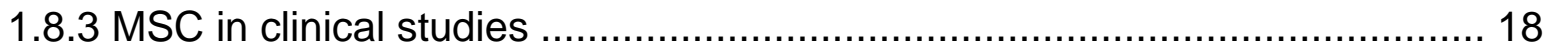

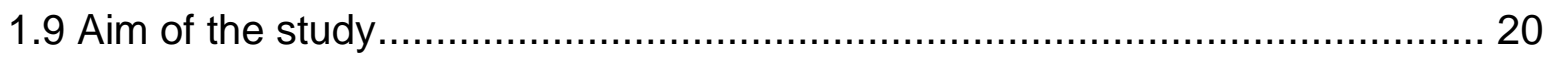

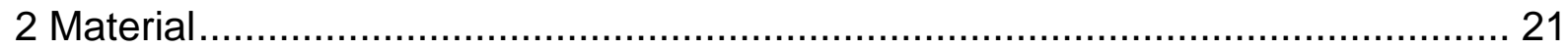

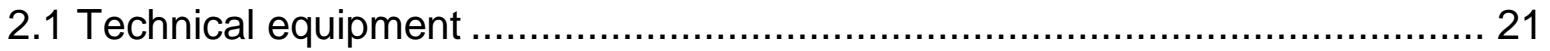

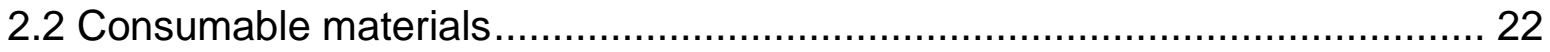

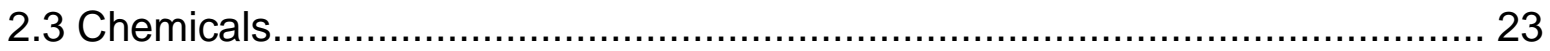

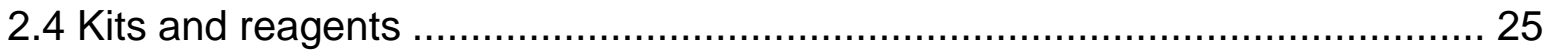

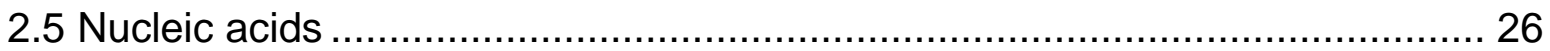

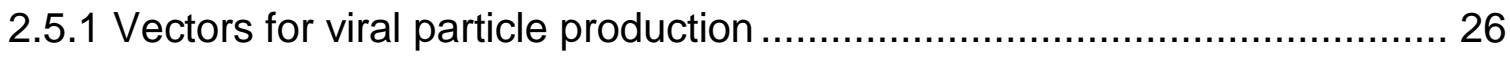

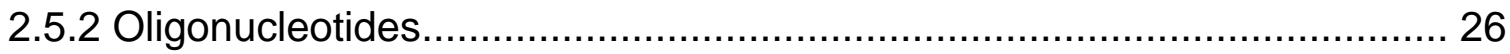

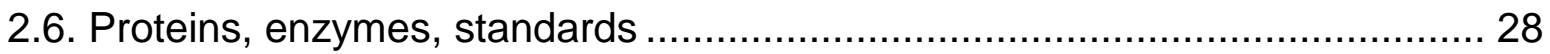

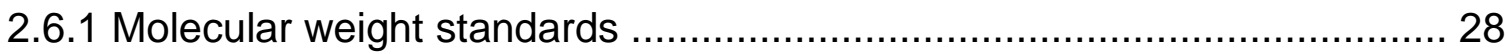




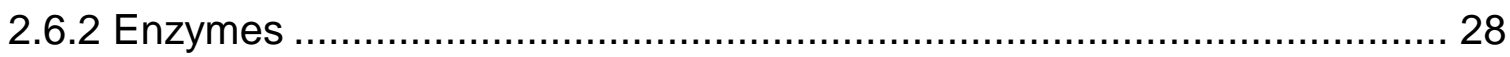

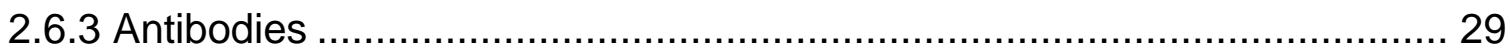

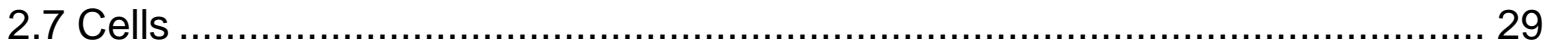

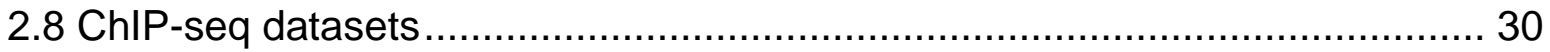

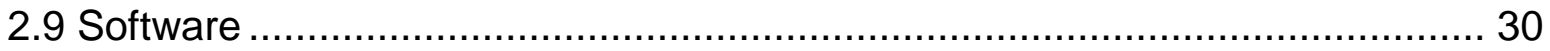

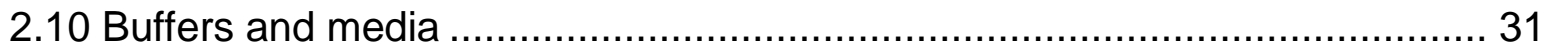

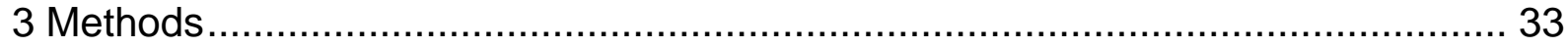

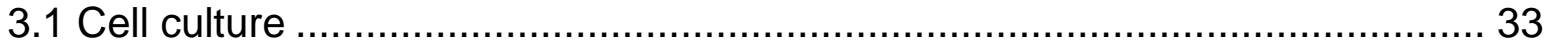

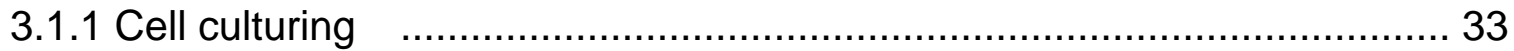

3.1.2 Adipocyte and osteoblast differentiation.............................................. 33

3.1.3 Reverse transfection .................................................................. 33

3.1.4 Forward transfection ............................................................... 34

3.1.5 Generation of stable cell lines by lentiviral infection ............................... 34

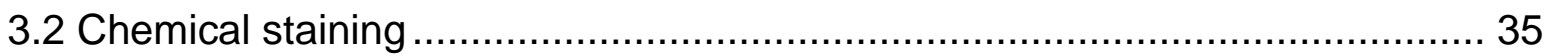

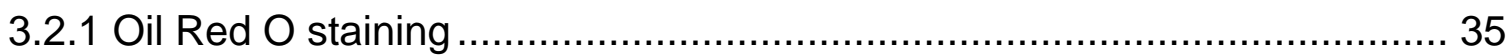

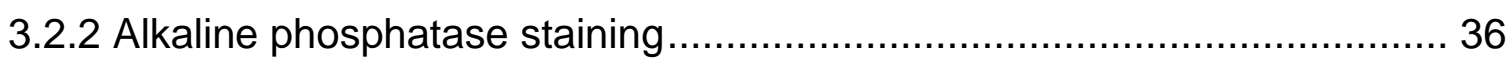

3.3 Ectopic bone formation experiment ……............................................. 36

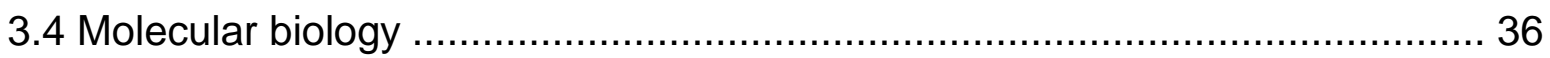

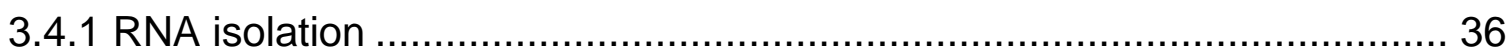

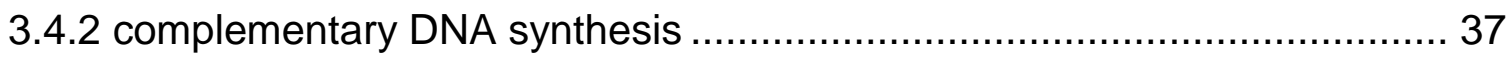

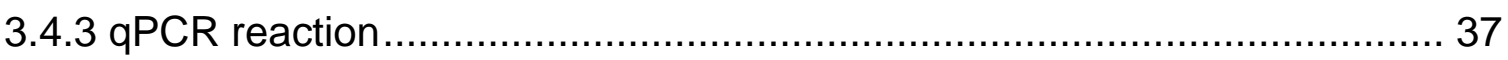

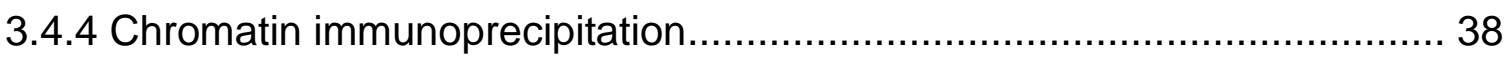

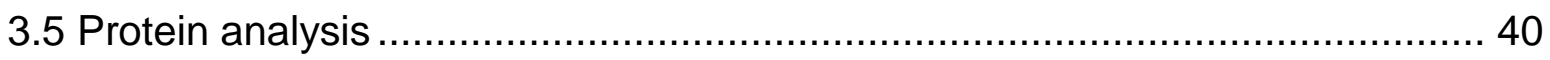

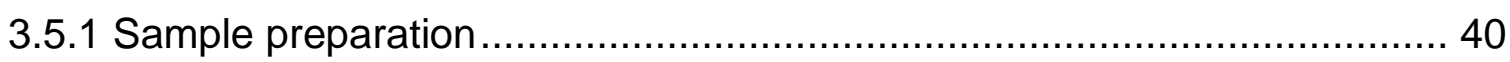

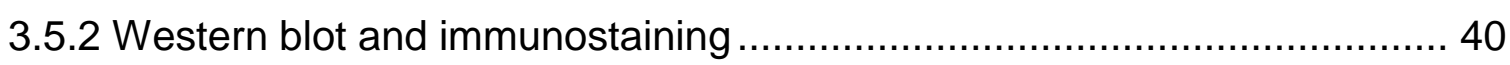

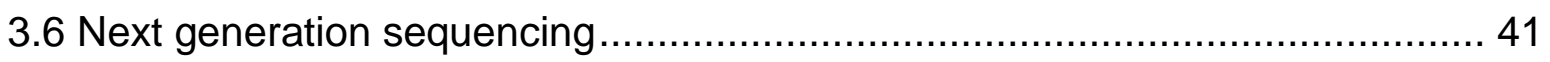

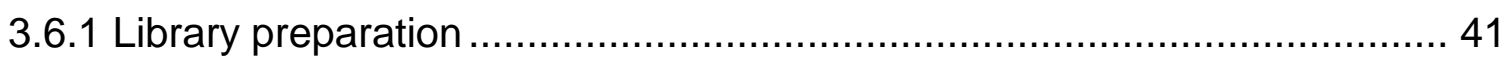

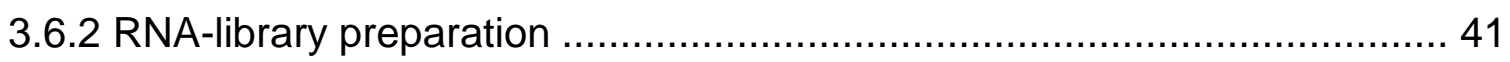

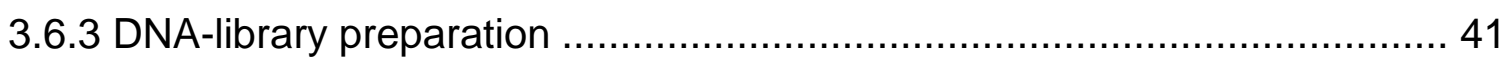

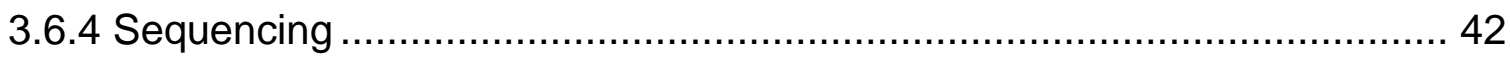

3.7 Bioinformatic processing of sequencing data ........................................... 42

3.7.1 Mapping of ChIP-seq reads to the genome........................................ 42

3.7.2 Peak calling via MACS2 ................................................................. 42

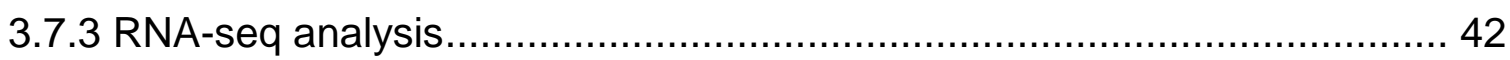

3.7.4 Normalization and calculation of ChIP-seq binding affinities................... 43

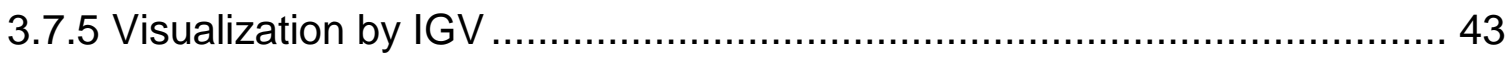




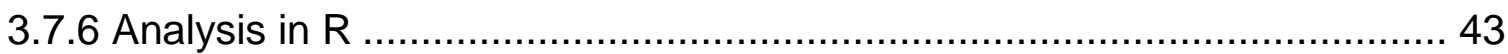

3.7.7 Cis-regulatory element annotation system ……....................................... 43

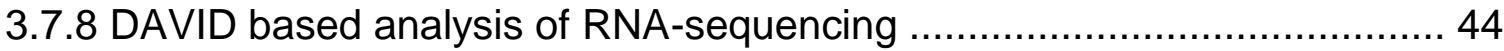

3.7.9 DiffBind and calculation of RNA-Pol II stalling ratios .............................. 44

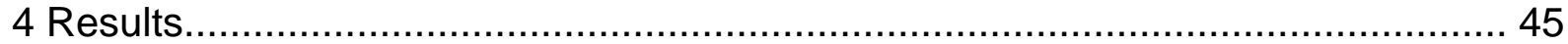

4.1 CHD1 depletion impairs MSC and FOB differentiation ................................ 45

4.2 Cell lineage specific genes are deregulated with reduced CHD1 levels .......... 48

4.3 Transcriptomic differences in MSC after CHD1 depletion and differentiation .. 49

4.4 CHD1 depletion attenuates regulation of differentiation regulated genes........ 51

4.5 Low transcribed genes require CHD1 for their activation during differentiation 53

4.6 CHD1 regulated genes are enriched in osteoblastic gene ontology terms ..... 54

4.7 Validation of single genes identified by RNA-seq with individual CHD1-siRNAs

4.8 Heterozygous CHD1 knockout mice show an abnormal bone phenotype ....... 61

4.9 CHD1 depleted MSC form less ectopic bone in mice .................................... 63

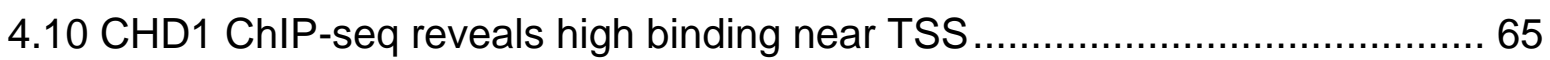

4.11 Genome-wide CHD1 binding is broadly associated with gene regions ......... 67

4.12 CHD1 correlates with active histone modifications and gene expression...... 69

4.13 Induced genes during differentiation are enriched in CHD1 binding around

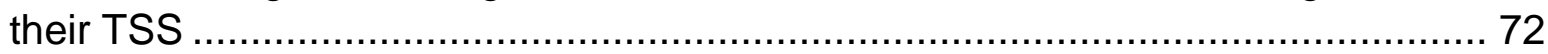

4.14 Single gene analysis reflects genome-wide observed effects ................... 75

4.15 Global RNA-Pol II occupancy increases around TSS after CHD1 depletion . 77 4.16 CHD1 dependent genes show unchanged RNA-Pol II levels around their TSS

4.17 ChIP-seq profiles reveal RNA-Pol II stalling also on single gene level 83

4.18 Highest RNA-Pol II stalling ratios at TSS-regions of CHD1 dependent genes

5 Discussion

5.1 Genome-wide transcription effects of CHD1 by regulating RNA-Pol II stalling 89

5.1.1 How is CHD1 depletion affecting gene expression in particular? 89

5.1.2 Reduced H2A.Z levels upon CHD1 depletion may link to decreased nucleosome turnover.

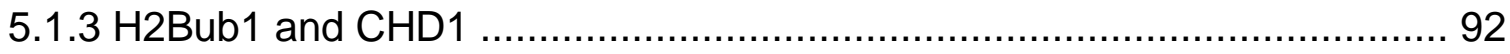

5.2 Genome-wide binding pattern of CHD1 ……........................................... 92

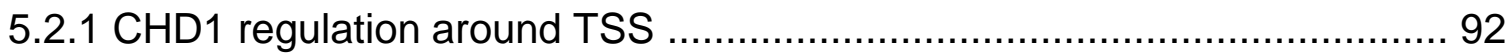

5.2.2 How is CHD1 recruited to differentiation-induced genes? ....................... 93

5.2.3 When is CHD1 recruited to differentiation-induced genes?..................... 93 
5.2.4 Regulatory roles of CHD1 at gene bodies ........................................ 94

5.3 Biological implications on osteoblast differentiation upon CHD1 depletion ..... 97

5.3.1 CHD1 regulation of bone development associated gene ontology terms .. 97

5.3.2 Ectopic bone formation and effects in vivo based on CHD1 ................... 98

5.3.3 Expression of $A L P L$ and BGLAP in osteogenesis after CHD1 depletion... 99

5.4 Translation for clinical therapeutic approaches ......................................... 99

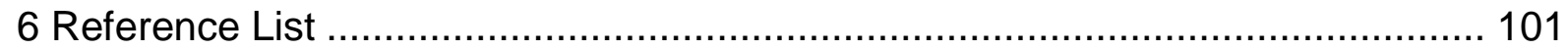

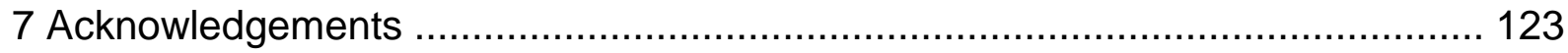




\section{Abbreviations}

${ }^{\circ} \mathrm{C} \quad$ degree Celsius / centrigrade

A ampere

Adi adipocyte

ALPL alkaline phosphatase, liver/bone/kidney

AR androgen receptor

ATP adenosine triphosphate

BA bone area

BA/ TA bone area/ total area

BAM binary alignment/map

Bejamini Bejamini-Hochberg procedure

BGLAP bone gamma-carboxyglutamate (gla) protein

BGP $\quad \beta$-glycerophosphate

BMP4 bone morphogenetic protein 4

bp base pair

BSA bovine serum albumin

CBP CREB-binding protein

cDNA completentary DNA

CEAS cis-regulatory element annotation system

CHD1 chromodomain helicase DNA binding protein 1

ChIP chromatin immunoprecipitation

ChIP-seq ChIP with subsequent high-throughput sequencing

$\mathrm{cm} \quad$ centimetre

$\mathrm{CO}_{2} \quad$ carbon dioxide

COL11A1 collagen, type $\mathrm{XI}$, alpha 1

CRC chromatin remodeling complex

CTCF CCCTC-binding factor

CTD carboxy-terminal domain

CTGF connective tissue growth factor

Da Dalton

DBD DNA-binding domain

$\mathrm{dd}_{2} \mathrm{O} \quad$ double destilled water

DKK1 dickkopf Wnt signaling pathway inhibitor 1 
DMEM Dulbecco's modified eagle's medium

DMSO dimethyl sulfoxide

DNA deoxyribonucleid acid

dNTP deoxyribonucleotide

DTT dithiothreitol

DUSP1 dual specificity phosphatase 1

DUSP5 dual specificity phosphatase 5

e.g. exempli gratia $=$ for example

ECM extra cellular matrix

EDN1 endothelin 1

EDTA ethylenediaminetetraacetic acid

ELN elastin

EMP3 epithelial membrane protein 3

ENCODE encyclopedia of DNA elements

ESC embryonic stem cell

et al. et alii $=$ and others

$\mathrm{EtOH}$ ethanol

expr. expression

$\mathrm{F} \quad$ forward

FACT facilitates chromatin transcription

FBS fetal bovine serum

FC fold change

FDR false discovery rate

Fig figure

FOB fetal osteoblast

g gravity $\left(9.81 \mathrm{~m} / \mathrm{s}^{2}\right)$

GAPDH glyceraldehyde-3-phosphate dehydrogenase

GB gene body

h hour

H\&E hematoxylin and eosin

$\mathrm{H} 1 \quad$ histone 1

$\mathrm{H} 2 \mathrm{~A} \quad$ histone $2 \mathrm{~A}$

H2A.Z H2A histone family, member Z

H2B histone 2B 
H2Bub1 histone H2B monoubiquitination at lysine 120

H3 histone 3

H3.3 H3 histone, family 3

H3K27ac histone H3 acetylated at lysine 27

H3K27me3 histone H3 trimethylation at lysine 27

H3K36me3 histone H3 trimethylation at lysine 36

H3K4me1 histone H3 monomethylation at lysine 4

H3K4me2 histone H3 dimethylation at lysine 4

H3K4me3 histone $\mathrm{H} 3$ trimethylation at lysine 4

$\mathrm{H} 4 \quad$ histone 4

HA hydroxyapatite

HAT histone acetyltransferase

HDAC histone deacetylase

hg19 human genome project version 19

HMT histone methyltransferase

HRP horseradish peroxidase

hs homo sapiens

HSC70 heat shock 70kDa protein 8

IBSP integrin-binding sialoprotein

IGF insulin-like growth factor 1

IgG immunoglobulin $\mathrm{G}$

IGV integrative genomics viewer

INO80 INO80 complex subunit

iPSC induced pluripotent stem cells

ISWI imitation SWI

$\mathrm{K} \quad$ lysine residue

$\mathrm{kb} \quad$ kilo base pairs

kDa kilo Dalton

kg kilogram

$\mathrm{LiCl} \quad$ lithium chloride

$\log \quad$ logarithm

LPL lipoprotein lipase

m milli $\left(10^{-3}\right)$

M methionine residue or molar, $\mathrm{mol} / \mathrm{L}$ 


$\begin{array}{ll}\text { MACS } & \text { model-based analysis of ChIP-seq } \\ \text { MED1 } & \text { mediator complex protein-1 } \\ \text { min } & \text { minute } \\ \text { mRNA } & \text { messenger RNA } \\ \text { MSC } & \text { mesenchymal stem cell } \\ \text { n } & \text { number of indivual values } \\ \text { n } & \text { nano (10-9) } \\ \text { n.s. } & \text { non-significant } \\ \text { NDR } & \text { nucleosome depleted region } \\ \text { NEM } & \text { N-ethylmaleinmide } \\ \text { NF-KB } & \text { nuclear factor of kappa B cells } \\ \text { NP-40 } & \text { nonidet P-40 } \\ \text { NuRD } & \text { nucleosome remodeling deacetylase } \\ \text { Ost } & \text { osteoblast } \\ \text { p } & \text { probability } \\ \text { P/S } & \text { penicillin/streptomycin } \\ \text { P300 } & \text { E1A binding protein p300 } \\ \text { PBS } & \text { phosphate buffered saline } \\ \text { PC } & \text { principle component } \\ \text { PCA } & \text { principle component analysis } \\ \text { PCIA } & \text { phenol:chloroform:isoamyl alcohol } \\ \text { PCR } & \text { polymerase chain reaction } \\ \text { pH } & \text { potentia hydrogenii = potential of hydrogen } \\ \text { PLIN1 } & \text { perilipin 1 } \\ \text { POSTN } & \text { periostin } \\ \text { PPARG } & \text { peroxisome proliferator-activated receptor gamma } \\ \text { PRC } & \text { polycomb repressive complex } \\ \text { ptm } & \text { post translational modification } \\ \text { qPCR } & \text { quantitative real-time PCR } \\ \text { R } & \text { arginine residue or reverse or Pearson correlation } \\ \text { RAR } & \text { coefficient } \\ \text { RIN } & \text { retinoic acid receptor } \\ \text { RING } & \text { RNA integrity number } \\ \text { reallyinteresting new gene } \\ \end{array}$




\begin{tabular}{|c|c|}
\hline RNA & ribonucleic acid \\
\hline RNA-Pol II & RNA polymerase II \\
\hline RNA-seq & sequencing of rt-transcribed RNA \\
\hline RPKM & reads per kilo base per million mapped reads \\
\hline RPLPO & ribosomal protein, large, P0 \\
\hline RSC & chromatin structure remodeling complex \\
\hline $\mathrm{RT}$ & room temperature \\
\hline RT-PCR & reverse transcription PCR \\
\hline RUNX2 & runt-related transcription factor 2 \\
\hline $\mathrm{s}$ & second \\
\hline s.d. & standard deviation \\
\hline SAGA & spt-ada-gcn5 acetyltransferase \\
\hline SAM & sequence alignment/map \\
\hline SCID & severe combined immunodeficiency \\
\hline SDS & sodium dodecylsulfate \\
\hline SDS-PAGE & SDS polyacrylamide gel electrophoresis \\
\hline shCHD1 & shRNA directed against CHD1 \\
\hline shCon & shRNA non-targeting \\
\hline shRNA & short hairpin RNA \\
\hline siCHD1 & siRNA directed against CHD1 \\
\hline siCon & siRNA control \\
\hline SiRNA & short interfering RNA \\
\hline SIX1 & SIX homeobox 1 \\
\hline SP7 & sp7 transcription factor \\
\hline SPP1 & osteopontin \\
\hline SSRP1 & structure specific recognition protein 1 \\
\hline SUMO & small ubiquitin-like modifier \\
\hline SWI/SNF & SWltch/sucrose nonfermentable \\
\hline TA & tissue area \\
\hline Taq & Thermus aquaticus \\
\hline TBP & TATA-binding protein \\
\hline $\mathrm{TCP}$ & tri-calcium phosphate \\
\hline TEMED & $\mathrm{N}, \mathrm{N}, \mathrm{N}^{\prime}, \mathrm{N}^{\prime}$-tetramethylethylenediamine \\
\hline TFF1 & trefoilfactor 1 \\
\hline
\end{tabular}




\section{TFIIA/B transcription factor $A / B$}

TGF $\beta$ transforming growth factor, beta

Tris Tris(hydroxymethyl)aminomethane

TSS transcriptional start site

TTS transcriptional termination site

U unit (enzyme activity)

ub ubiquitin

un unregulated

Und undifferentiated

up up regulated

UTR untranslated region

$\mathrm{V} \quad$ voltage

$\mathrm{v} / \mathrm{v} \quad$ volume per volume

VDR vitamin $D(1,25$ - dihydroxyvitamin $D 3)$ receptor

VIM vimentin

vs. versus

w/v weight per volume

wt wild type

a alpha

$\beta \quad$ beta

Y gamma

$\mu \quad \operatorname{micro}\left(10^{-6}\right)$ 


\section{List of Figures}

FIGURE 1: ORGANIZATION OF DNA FROM DECONDENSED (TOP) TO HIGHER

CONDENSED (BOTTOM) CHROMATIN STRUCTURES.

FIGURE 2: POST-TRANSLATIONAL HISTONE MODIFICATIONS IN HUMANS.... 3

FIGURE 3: DIFFERENT OUTCOMES OF NUCLEOSOME SLIDING.

FIGURE 4:CHD1 DOMAINS AND ITS INTERACTIONS NEAR THE

TRANSCRIPTIONAL START SITE IN MAMMALS................................... 14

FIGURE 5: EPIGENETIC LANDSCAPE MODEL BY WADDINGTON. ................. 15

FIGURE 6: CHD1 DEPLETION ALTERS ADIPOCYTE AND OSTEOBLAST

DIFFERENTIATION IN MSC

FIGURE 7: OSTEOBLAST DIFFERENTIATION MARKER ARE REDUCED AFTER

CHD1 DEPLETION IN FOB.

FIGURE 8: DIFFERENTIATION AND CHD1 DEPLETION CAUSES BROAD

TRANSCRIPTOMIC CHANGES.

FIGURE 9: GENES REGULATED DURING DIFFERENTIATION ARE

ATTENUATED UPON CHD1 DEPLETION.

FIGURE 10: CHD1 IS NECESSARY FOR INDUCED CHANGES IN GENE

EXPRESSION DURING OSTEOGENESIS.

FIGURE 11: INDIVIDUAL CHD1-SIRNAS VALIDATE CHD1-SPECIFIC GENE

REGULATION OBSERVED BY RNA-SEQ.

FIGURE 12: CHD1 HETEROZYGOUS KNOCKOUT MICE SHOW DECREASED

BONE ASSOCIATED PARAMETERS.

FIGURE 13: CHD1 DEPLETION REDUCES ECTOPIC BONE FORMATION IN

MICE.

FIGURE 14: CHIP-QPCR AND CHIP-SEQ PROFILES OF CHD1 AND H3K4ME3

OVERLAP AROUND TSS. 66

FIGURE 15: CHD1 DISTRIBUTION ON GENOMIC ELEMENTS.

FIGURE 16: CHD1 POSITIVELY CORRELATES WITH ACTIVE HISTONE MARKS

AND GENE EXPRESSION.

FIGURE 17: CHD1 BINDING IS ENRICHED NEAR THE TSS-REGIONS OF

INDUCED GENES DURING MSC DIFFERENTIATION.

FIGURE 18: CHD1 BINDING SIGNALS INCREASE AROUND TSS DURING

OSTEOGENESIS AT CHD1 DEPENDENT GENES. 
FIGURE 19: CHD1 DEPLETION INCREASES RNA-POL II AND DECREASES

H2A.Z BINDING AROUND TSS.

FIGURE 20: UNCHANGED RNA-POL II OCCUPANCY AROUND TSS OF

REPRESSED GENES AFTER CHD1 DEPLETION.

FIGURE 21: SINGLE-GENE PROFILES DESCRIBE DIFFERENT PATTERN OF

RNA-POL II PROFILES AFTER CHD1 DEPLETION.

FIGURE 22: RNA-POL II STALLING RATIOS ARE HIGHEST IN REPRESSED

GENES AFTER CHD1 DEPLETION.

FIGURE 23 MODEL OF CHD1-REGULATED GENE EXPRESSION DURING

DIFFERENTIATION OF INDUCED AND STABLY TRANSCRIBED, NONINDUCED GENES. 


\section{Summary}

Nucleosome remodeling, histone modifications and exchange of histone variants are interconnected mechanisms involved in regulation of gene transcription. Nucleosomes can act as strong barriers and are remodeled during RNA-Pol IImediated transcription elongation. Remodeling of nucleosomes is tightly regulated in particular during activation and inhibition of cellular differentiation. The nucleosome remodeler CHD1 is a transcriptional co-activator involved in RNA-Pol II processivity downstream of the transcriptional start site (TSS). In this study we hypothesized that CHD1 not only acts as a general co-activator of transcription but can also regulate gene specific expression. Therefore, we investigated the role of $\mathrm{CHD} 1$ on gene regulation after induction of adipocyte and osteoblast differentiation.

Genome-wide binding analysis of CHD1 during differentiation revealed high occupancy at TSS-regions of adipocyte and osteoblast activated genes. Further we observed direct regulation of these activated genes by enriched CHD1 binding around TSS. Concordantly, CHD1 was required for ectopic bone formation in mice. Besides these biological aspects it could here be shown that global RNA-Pol II stalling downstream of TSS was caused by CHD1 depletion. This highlights its genomic role for efficient early RNA-Pol II-mediated transcription elongation. A group of highly activated genes during osteoblast differentiation was found to be repressed by significantly increased RNA-Pol II stalling ratios in parallel with decreased CHD1 protein levels. Interestingly, high steady-state levels of the histone variant H2A.Z at the TSS-region were revealed to be dependent on CHD1. This presumably increases the nucleosome stability and thus cause the observed global RNA-Pol II stalling. Summarized, CHD1 was shown to be necessary for a genome-wide, efficient RNAPol II-mediated early transcription elongation, probably achieved by decreasing the nucleosome barrier at the TSS-region. In particular, CHD1 was required for the activation of a group of genes involved in osteoblast differentiation. This implies a function for CHD1 as a regulatory protein in cell differentiation. Further we propose that CHD1 should be considered in quality control of MSC in skeletal stem cell therapies. 


\section{Introduction}

\subsection{DNA organization}

The approximately $2 \mathrm{~m}$ long human DNA is highly compacted and organized in an approximately $10 \mu \mathrm{m}$ diameter sized nucleus within a dynamic structure called chromatin (Alberts B, Johnson A, Lewis J, et al., 2002). The chromatin consists of DNA, protein and RNA. Besides packaging and protecting the DNA, it also regulates the DNA accessibility required for cellular processes such as transcription and replication. The basic structural unit of chromatin is the nucleosome which is defined as $146 \mathrm{bp}$ of DNA wrapped around an octamer of 4 core histone proteins consisting of $\mathrm{H} 2 \mathrm{~A}, \mathrm{H} 2 \mathrm{~B}, \mathrm{H} 3$ and $\mathrm{H} 4$ (Luger et al., 1997). Two H2A/H2B heterodimers connect with two $\mathrm{H} 3 / \mathrm{H} 4$ heterodimers to form a stable histone octamer (Kornberg, 1974). These interact via a "hand-shake motif" and connect in the presence of DNA. In addition to the core particles, the histone $\mathrm{H} 1$ binds to linker DNA between two nucleosomes. This further stabilizes the DNA wrapped around the nucleosome and helps to fold the higher compacted chromatin (Allan et al., 1986; Hansen, 2002).

The differences in the compaction of chromatin can be differentiated within a cell and associates with different functions (Figure 1). After the nucleosome, the next level of compaction is the $10 \mathrm{~nm}$ fiber or "beads on a string" structure, which defines open accessible chromatin for example at transcriptional start sites or regulatory sequences (Cooper, 2000). Further packaging includes the $30 \mathrm{~nm}$ and $300 \mathrm{~nm}$ fibers, which both represent highly compacted chromatin (Tremethick, 2007). Though, the $30 \mathrm{~nm}$ fiber can be actively remodeled to the open $10 \mathrm{~nm}$ fiber and is associated with actively transcribed genes (Li et al., 2010), the 300 or $700 \mathrm{~nm}$ structure characterizes condensed chromatin which is present in the interphase of mitosis.

In general, two states of chromatin are distinguished, the eu- and heterochromatin. Euchromatin represents 10 to $30 \mathrm{~nm}$ thick fibers which characterize open and accessible chromatin. Cellular processes which involve the direct contact with DNA such as transcription, DNA-repair and -replication require euchromatin (Cooper, 2000). Heterochromatin in contrast defines closed and condensed chromatin, located most often at the centromere or telomere with repetitive elements (Grewal and Jia, 2007). 


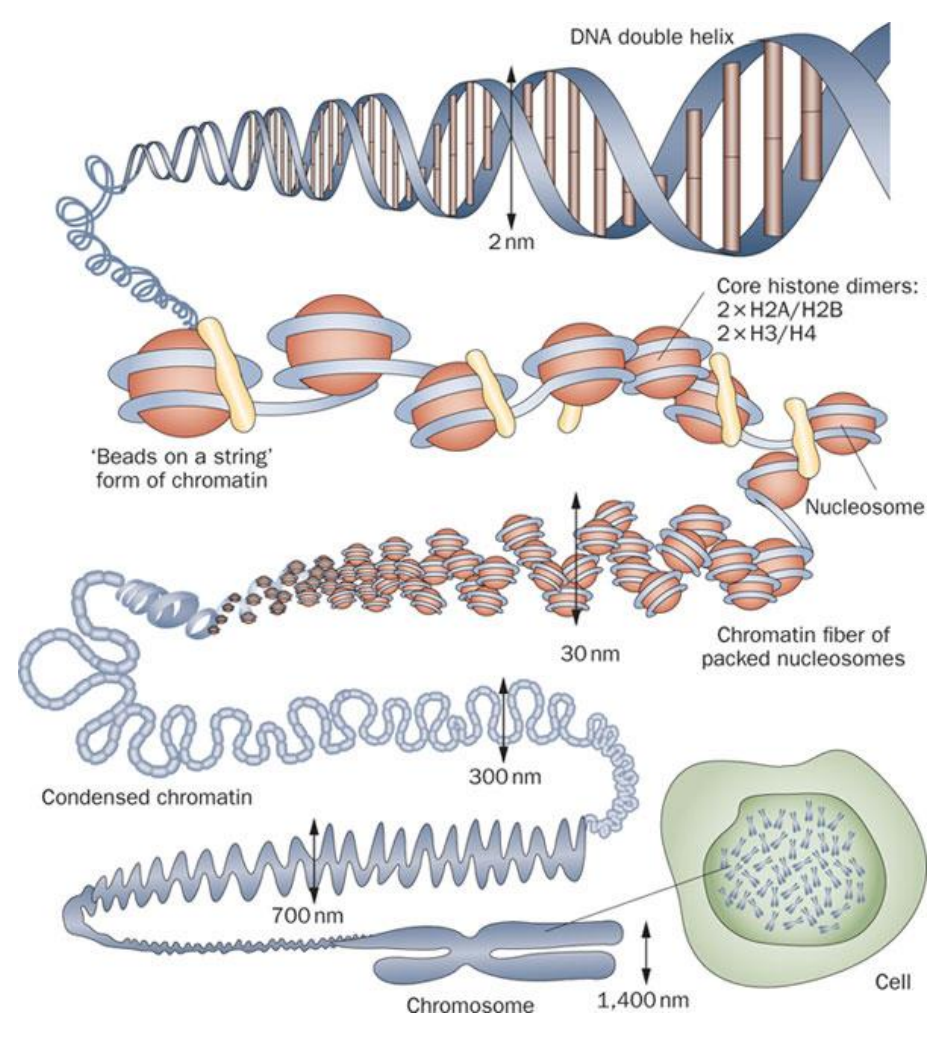

Figure 1: Organization of DNA from decondensed (top) to higher condensed (bottom) chromatin structures. In the lowest condensed chromatin state, the "beads on a string" structure is the DNA wrapped around a histone octamer associated with histone $\mathrm{H} 1$ (yellow). Further compression includes $30 \mathrm{~nm}, 300 \mathrm{~nm}$ and $700 \mathrm{~nm}$ chromatin structures which leads to the organization of interphase chromosome within a cell nucleus (Figure taken from Tonna et al., 2010).

\subsection{Histone modifications}

Negatively charged DNA and positive charged histones are tightly bound when forming the nucleosome, however $\mathrm{N}$ - and $\mathrm{C}$-terminal tails of histones protrude from nucleosomes and are often targets of different post-translational modifications (PTM) (Luger et al., 1997; Van Holde et al., 1980). However, some histone amino acids lying within the core of nucleosomes between the tails and get modified, too (Tropberger et al., 2013). The most common PTMs include acetylation, methylation, phosphorylation, ubiquitination and SUMOylation (Figure 2) (Bannister and Kouzarides, 2011). The modifications consist of relatively small, covalently bound acetyl-, phospho- or methyl-groups up to relatively large protein moieties like ubiquitin or Small Ubiquitin-like Modifier (SUMO) attached to specific amino acids of histones. Interestingly, the high variability in PTMs leads to extensive changes in the chromatin, mainly either loosening DNA-histone interactions and/or changing the binding properties with other proteins or complexes (Bannister and Kouzarides, 
2011). These changes have a broad impact on nearly all cellular processes, although cause and effect of histone modifications have to be considered carefully.

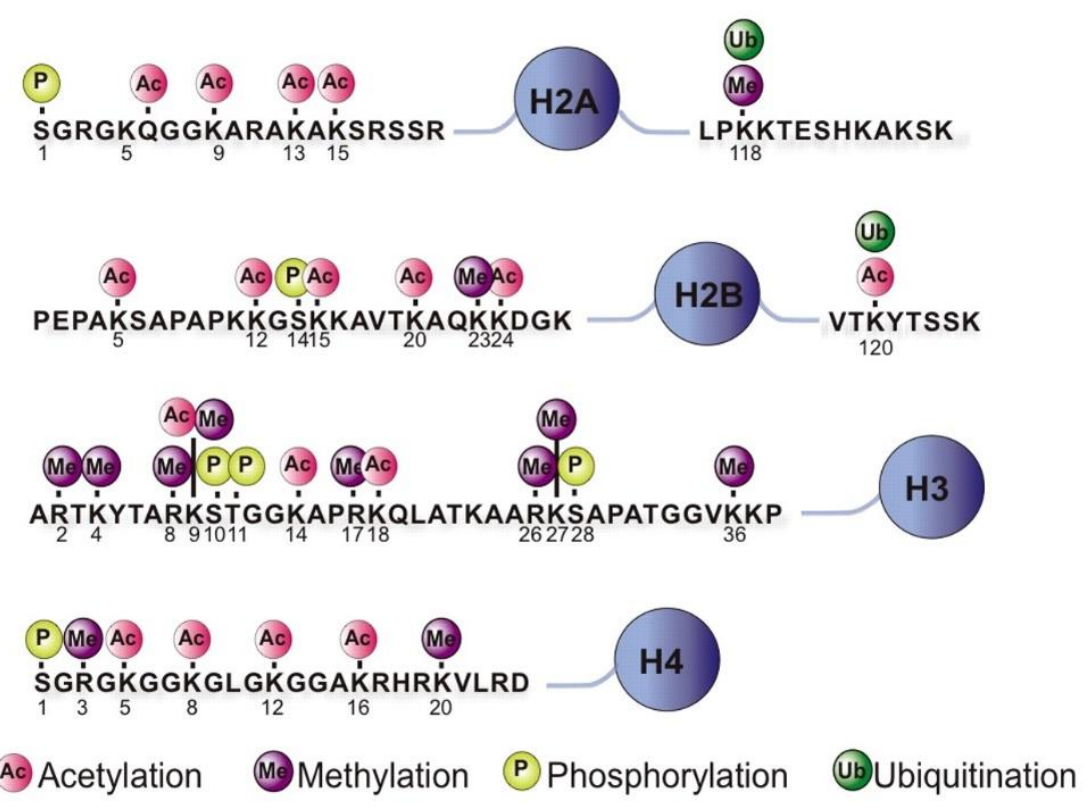

Figure 2: Post-translational histone modifications in humans. Schematic view of $\mathrm{N}$ - and $\mathrm{C}$ terminal tails of Histone $2 \mathrm{~A}, 2 \mathrm{~B}, 3$ and $4(\mathrm{H} 2 \mathrm{~A}, \mathrm{H} 2 \mathrm{~B}, \mathrm{H} 3, \mathrm{H} 4)$ shows post-translational histone modification (explained at bottom) at respective amino acid residues of each histone (Figure taken from Kato et al., 2010).

\subsection{Deciphering the "histone code"}

Extensive modifications on one nucleosome raised the hypothesis for a "histone code" stating the association of various epigenetic changes defining a single functional and regulatory event (Strahl and Allis, 2000). Since then, thousands of genome-wide chromatin immunoprecipitation with subsequent deep sequencing (ChIP-seq) studies were performed analyzing different histone modifications in different cell lines and organisms (Bernstein et al., 2010; ENCODE Project Consortium, 2012). Indeed, these great amount of data confirmed the early hypothesis and revealed that histone marks could characterize the active and repressed regions of genomic elements in a cell type-specific manner (Álvarez-Errico et al., 2015; Li et al., 2007). These data also revealed that active transcriptional start sites (TSS) in eukaryotes are frequently marked by histone $\mathrm{H} 3$ tri-methylated at lysine 4 (H3K4me3) and histone $\mathrm{H} 3$ acetylated at lysine 27 (H3K27ac). Actively transcribed gene bodies show marks like monoubiquitinated H2B (H2Bub1) and H3K36me3. In contrast, inactive and repressed gene regions are frequently characterized by H3K27me3 marks, whereas condensed chromatin is typically 
associated with H3K9me3. Furthermore, co-occupancy of functionally active and repressive histone modifications like H3K4me3 and H3K27me3 describe a specific subset of genes, called bivalent genes. Bivalent genes are in a transcriptionally inactive state, but often occupied already with RNA-Polymerase II (RNA-Pol II), and ready to be transcribed very fast after a certain stimulus (Bernstein et al., 2006; Min et al., 2011). These genes are often involved in regulation of differentiation of developmental processes which can induce further cell fate committing genes. It has been shown that especially undifferentiated cells such as embryonic stem cells tend to have genes in a bivalent state (Grandy et al., 2015; Ravens et al., 2015). In addition to its role in transcription regulation the histone code is associated with processes such as DNA-repair, histone exchange and dosage compensation (Heard and Disteche, 2006; Venkatesh and Workman, 2015; Zhu and Wani, 2010).

This altogether indicates that the "histone code" can be used to describe not only the genomic regions, but also regulatory processes and characteristic cellular functions. Moreover, since cells have characteristic histone modification "landscapes" this can be further used for cell type specification or even for predictions of gene expression (Heintzman et al., 2009; Heinz et al., 2015; Karlić et al., 2010; Koch et al., 2007).

\subsection{Histone variants}

Nucleosomes can be changed in their canonical histone composition by histone variants. Several conserved histone variants were described for each histone except $\mathrm{H} 4$ (Kamakaka and Biggins, 2005). $\mathrm{H} 2 \mathrm{~A}$ and $\mathrm{H} 3$ show the highest diversity in variants like H2A.X, H2A.Z, macro H2A, H2A.Bdb and for H3 like H3.1, H3.2, H3.3. These variants can change the interaction of DNA and proteins when replacing canonical histones within the nucleosomes (Bönisch and Hake, 2012). Furthermore, they also regulate nucleosome stability or occupancy of modifications (Kamakaka and Biggins, 2005). The functionality and homology of histone variants compared to their canonical counterparts vary greatly. For example, the histone variant $\mathrm{H} 3.3$ is almost identical to the $\mathrm{H} 3$ and differs only in 4 or 5 amino acids, whereas H2A.Z has only a $60 \%$ homology to H2A (Zlatanova and Thakar, 2008). Additionally, histone variants can be associated with a common regulatory feature of cellular processes as known for H2A.Z or H3.3 in transcription (Jin et al., 2009). Interestingly, some of them are also associated with cell type-specific functions like the histone variant $\mathrm{H} 3.5$ in sperm and testis (Schenk et al., 2011). Moreover, they can also differ in their occupancy 
within genome. H2A.Bdb is restricted to autosomes and the active X-chromosome, whereas macro H2A is mainly found in the inactive X-chromosome (Chadwick and Willard, 2001; Costanzi and Pehrson, 1998).

\subsubsection{Histone variant $\mathrm{H} 3.3$}

H3.3 can be incorporated into nucleosomes in a replication-independent manner and is mainly associated with active promoters, gene bodies or enhancer regions (Ahmad and Henikoff, 2002; Jin et al., 2009; Sarai et al., 2013). A study showed that H3.3 containing nucleosomes are more sensitive to low salt concentrations and are less stable than those with the canonical histone H3 (Jin and Felsenfeld, 2007). Concordant with these earlier studies it was also shown that $\mathrm{H} 3.3$ causes higher nucleosome turnover, a characteristic believed to keep the DNA in a transiently accessible state for regulation (Mito et al., 2005; Wirbelauer et al., 2005). Interestingly, H3.3 appears also to be required for instance for the maintenance of a specific chromatin landscape in embryonic stem cells by associating with the polycomb repressive complex 2 (PRC2) complex mediated by H3K27me3, despite high nucleosome turnover still being present (Banaszynski et al., 2013). Furthermore, in $31 \%$ of pediatric glioblastoma samples H3.3 was shown to be mutated at sites corresponding to H3K27, the modification which is well known to play an important role in gene regulation (Schwartzentruber et al., 2012).

\subsubsection{Histone variant H2A.Z}

$\mathrm{H} 2 \mathrm{~A} . \mathrm{Z}$ is a highly conserved protein present in the protozoan Plasmodium falciparum, Saccharomyces cerevisiae and human with approximately $90 \%$ sequence conservation (louzalen et al., 1996; Zlatanova and Thakar, 2008). In the nucleosome H2A.Z can form a heterodimer with H2B in either a homotypic (H2A.Z/ H2A.Z) or heterotypic (H2A/ H2A.Z) fashion and thus alter the interaction with the $\mathrm{H} 3 / \mathrm{H} 4$ heterotetramer (Suto et al., 2000). Though the overall nucleosome structure is less changed to its canonical counterpart, the homotypic H2A.Z-containing nucleosomes are more sensitive to low salt concentrations and less stable (Weber et al., 2010). Interestingly, H2A.Z was found to be enriched in mammals and $S$. cerevisiae at the +1 nucleosome, the first nucleosome downstream of the TSS, which is thought to have regulatory roles in transcription by forming a barrier for RNA-Pol II (Bönisch and Hake, 2012; Zhang et al., 2005). However, besides its broadly described association 
with gene activation H2A.Z has been correlated also with gene repression (Marques et al., 2010). Therefore, H2A.Z is rather thought to affect nucleosome positioning by sliding (nucleosome sliding described below) than solely reducing nucleosome stability and decreasing the hurdle for RNA-Pol II (Albert et al., 2007; Bönisch and Hake, 2012; Guillemette et al., 2005). These features link them to a broad range of cellular processes such as DNA-repair, transcription regulation and segmentation of euchromatin and heterochromatin (Bönisch and Hake, 2012; Meneghini et al., 2003).

Interestingly, H3.3 and H2A.Z histone variants often co-occupy positions at the +1 nucleosome and may promote a decreased nucleosomal barrier for RNA-Pol II around the TSS (Jin et al., 2009). Concordant with that, a previous study showed that nucleosomes with H2A.Z and H3.3 were even more sensitive to low salt concentrations, however another group found only subtle differences in their stability, but increased variability in the nucleosome positioning (Jin and Felsenfeld, 2007; Thakar et al., 2009). With the exception of TSS, additional regulatory regions are cooccupied with the two histones variants such as enhancer and insulator regions supporting their role in the maintenance of DNA accessibility (Chen et al., 2014b; Jin et al., 2009).

\subsection{Nucleosome remodeling}

Another mechanism of chromatin rearrangement is the remodeling and movement of nucleosomes. Nucleosome remodeling and positioning were shown to be important in transcription, DNA-repair or DNA-recombination (Green and Almouzni, 2002; Kamakaka and Thomas, 1990; Roth and Roth, 2000). Although the DNA sequence is a strong indicator for nucleosome positioning, half of the human genome is covered by regularly spaced nucleosomes and around $10 \%$ of them show highly consistent positioning, which indicates a genome-wide role of nucleosome remodeling (Gaffney et al., 2012). In general, four nucleosome remodeling mechanisms are described: i) sliding of nucleosomes by an ATP-dependent remodeler, ii) ejection of a complete histone octamer, iii) replacement or iv) removal of the H2A/ H2B histones from a nucleosome (Cairns, 2007).

\subsubsection{Nucleosome sliding}

Nucleosome sliding is performed by enzymes which are closely related to helicases and characterized by a highly conserved ATPase domain. The exact molecular 
mechanism how nucleosome sliding is performed is still a matter of debate (MuellerPlanitz et al., 2013). However, one model predicts that the ATPase remodeler bind at two distal sites on the nucleosome and loosen the interaction between the DNA and histones. The free DNA is then directionally pulled from the nucleosome and is thought to form a loop. By this ATP-dependent process linker DNA is dragged behind which transfers around the nucleosome and finally results in a shift. The majority of ATP-dependent chromatin remodelers act in modular multi-protein complexes and are broadly expressed (Mueller-Planitz et al., 2013; de la Serna et al., 2006). These are classified into several families such as SWItch/Sucrose Non-Fermentable (SWI/SNF), imitation SWI (ISWI), chromodomain helicase DNA-binding (CHD), INO80 complex (INO80), etc. (Clapier and Cairns, 2009). Further, the modularity of the associated complexes connects them to a variety of cellular processes with spatial and temporal regulation like differentiation and development (Chi et al., 2003; Das et al., 2007; Reynolds et al., 2012).

In general, these chromatin remodeling complexes (CRC) can be divided into repressing or activating ones. For instance, the nucleosome remodeling deacetylase (NuRD) complex is a repressive CRC with multiple components like histone deacetylases-1 or 2 (HDAC1/2) and SNF2-ATPase domain containing chromodomain helicase DNA-binding protein-3/4 (CHD3/4) (Xue et al., 1998). In contrast, the SWI/SNF complex is an activating CRC with histone acetyl transferase activity (HAT) and AT-rich interactive domain 1A/B (ARID1A/B) (Kwon et al., 1994). Interestingly, active and repressive $\mathrm{CRC}$ often can have competitive roles in regulation of cell fates (Gao et al., 2009). However, CRC are difficult to analyze because of their modularity and often cell type specific functions (Voss and Hager, 2014).

Nucleosome sliding in general can cause three different outcomes: Nucleosome spacing, phasing or positioning (Figure 3) (Mueller-Planitz et al., 2013). Nucleosome spacing creates a regular array of nucleosomes which is often associated with dynamic and active chromatin (Gaffney et al., 2012). Approximately half of the human genome is covered by these arrays. It was shown in yeast that defects in nucleosome spacing due to depletion of a nucleosome remodeler led to cryptic transcription in the gene bodies (Hennig et al., 2012; Shim et al., 2012). Furthermore, regularly spaced 
arrays might be important for the integrity of chromatin fibers during DNA replication (Fletcher and Hansen, 1996).

Nucleosome phasing describes a patterning of nucleosomes relative to a nucleosome depleted region (NDR). NDRs are long-term open DNA regions caused by i) DNA sequences rich in AT which repels the association with histones, ii) due to the occupancy of DNA-bound proteins e.g. at the TSS or iii) ATP-dependent remodeling (Struhl and Segal, 2013). These regions are important regulatory sites which are required for the recruitment and binding of proteins like transcription factors or RNA-Pol II associated proteins (Venkatesh and Workman, 2015). The +1 nucleosome is a strong determinant for nucleosome phasing (Mavrich et al., 2008). Depletion of the Snf2-related nucleosome-spacing enzymes showed strong alterations in histone phasing after the +1 nucleosome reaching into the gene body(Gkikopoulos et al., 2011; Pointner et al., 2012). Surprisingly this had however weak influence on global transcription, but increased cryptic transcription. Interestingly, the +1 nucleosome is the well positioned nucleosome and belongs to the $10 \%$ of the most consistently positioned nucleosome in the human genome (Gaffney et al., 2012). Furthermore, neither loss of histone chaperones (described below) nor the loss of ATP-dependent remodeler appears to regulate this solidly positioned nucleosome.

During nucleosome positioning, DNA sequences can be disclosed or exposed by interaction with histones within the nucleosomes and thus for example regulate an active or inactive state. In yeast, the chromatin structure remodeling complex (RSC), which is related the human SWI/SNF complex, maintains NDR, whereas the ATPdependent nucleosome remodeler Imitation Switch subfamily 2 (Isw2) was reported to shift nucleosomes at the TSS and thus inhibit transcriptional initiation (Badis et al., 2008; Whitehouse and Tsukiyama, 2006). 


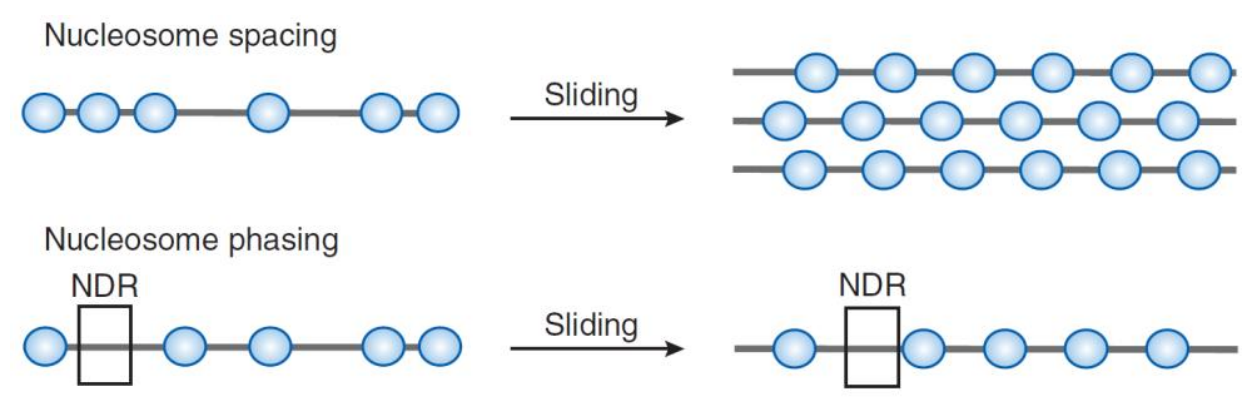

Nucleosome positioning

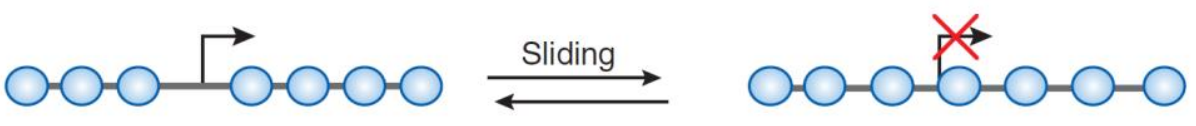

Figure 3: Different outcomes of nucleosome sliding. The model depicts nucleosomes (blue) on a DNA (grey line) in a decondensed status which are remodeled from left by respective mechanisms, as named top left, respectively. Black arrow on the nucleosome and DNA indicates transcriptional start site and its directionality, NDR - Nucleosome depleted region (Figure modified after Mueller-Planitz et al., 2013).

\subsubsection{Ejection and histone replacement/removal}

The canonical nucleosome is a stable structure, however, a dynamic exchange of histones occurs over various regions e.g. gene bodies (Das and Tyler, 2013). The main challenge during remodeling is to disturb the histone-histone or DNA-histone interactions within one nucleosome to remove or replace it. DNA-histone interactions are modified by ATP-dependent chromatin remodeler whereas histone-histone interactions are changed by histone chaperones (Gurard-Levin et al., 2014). These proteins often act together in complexes to unfold nucleosomes, as shown in yeast and humans (Cho et al., 2013; Okada et al., 2009; Simic et al., 2003).

\subsubsection{Histone dynamics in transcription}

Early studies on RNA-Pol II elongation has shown that nucleosomes can block transcription in vitro, whereas in vivo the nucleosomes are remodeled so that Polymerase can passage (Chang and Luse, 1997; Kireeva et al., 2002). During the process of remodeling the histone chaperones facilitates chromatin transcription (FACT) complex and Nef-associated protein 1 (Nap1) destabilize the interaction between the $\mathrm{H} 2 \mathrm{~A} / \mathrm{H} 2 \mathrm{~B}$ dimer and the $\mathrm{H} 3 / \mathrm{H} 4$ tetramer which causes the ejection of $\mathrm{H} 2 \mathrm{~A} / \mathrm{H} 2 \mathrm{~B}$ (Petesch and Lis, 2012). This is often sufficient for the RNA-Pol II to overcome the nucleosome barrier. Interestingly, H2Bub1 is stimulating the FACT dependent nucleosome remodeling and enhances the RNA-Pol II passage frequency 
(Pavri et al., 2006). This highlights the interplay on chromatin remodeling between histone modifications and CRC. Further, it also shows possible regulatory mechanisms of transcription by modulating histone dynamics over the gene body. Concordant with that, the chaperone specific recruitment to genes might play a role in fine tuning gene expression (Jimeno-González et al., 2006).

After RNA-Pol II passage, histones are reassembled to the DNA by FACT and ordered into a spaced array by ATP-dependent chromatin remodeler (Hsieh et al., 2013; Smolle et al., 2012). This might be a coordinated process between CHD1 and FACT as studies pointed out in yeast, Drosophila melanogaster and human by the interaction of both proteins (Krogan et al., 2002; Simic et al., 2003; Sims et al., 2007). Defects in either of both proteins were shown to increase cryptic transcription by RNA-Pol II (Carvalho et al., 2013; Smolle et al., 2012).

\subsubsection{Histone dynamics at the TSS}

Similar to gene bodies, nucleosomes at the transcriptional start sites are strong barriers for initiating RNA-Pol II in vitro (Lorch et al., 1987). After RNA-Pol II recruitment to the TSS and accomplished transcription of $20-50 \mathrm{bp}$ into the gene, often a pause follows (Sainsbury et al., 2015). This pausing RNA-Pol II requires further signaling to continue transcription into the gene body. Additionally, the +1 nucleosome needs to be overcome. Interestingly, this nucleosome position is slightly shifted downstream relative to the TSS when the RNA-Pol II is paused (Jonkers and Lis, 2015; Li and Gilmour, 2013; Weber et al., 2014). This "pushed" nucleosome by pausing RNA-Pol II indicates a transcriptional block. Further evidence for $a+1$ nucleosome dependent barrier in vivo was provided by decreased RNA-Pol II pausing at H2A.Z enriched genes, which destabilizes nucleosomes (described above). Concordantly, knockdown of H2A.Z caused higher stalling of RNA-Pol II around the TSS by decreasing the +1 nucleosome turnover (Weber et al., 2014). Thus, regulation of the +1 nucleosome barrier directly affects the gene transcription by RNA-Pol II.

Factors which are involved in the turnover of the nucleosome barrier at the TSS have been described previously. In particular, ATP-dependent chromatin remodeler, histone modifications or histone variants play a pivotal role (Skene et al., 2014; Svensson et al., 2015; Venkatesh and Workman, 2015). Hereby, the incorporation of 
$\mathrm{H} 2 \mathrm{~A} . \mathrm{Z}$ or $\mathrm{H} 3.3$ could affect the gene regulation by modulating the nucleosomal barrier. H2A.Z is incorporated into human nucleosomes either by the p400/TIP60 complex or the Snf2-related CREBBP activator protein (SCRAP), which are both ATP-dependent remodelers (Billon and Côté, 2013). In contrast, H3.3 is incorporated into nucleosomes by the histone cell cycle regulation defective homolog A (HIRA) chaperone (Ray-Gallet et al., 2002). Interestingly, impairment in H3.3 gene specific incorporation showed defects during development and differentiation rather than global transcription effects (Dutta et al., 2010; Szenker et al., 2012).

\subsection{CHD1}

The highly conserved chromodomain helicase DNA binding protein-1 (CHD1) is a ATP-dependent chromatin remodeler and belongs to the CHD family with 9 members (CHD1 - 9) (Marfella and Imbalzano, 2007). CHD1 has a tandem chromodomain at the N-terminal site, a central SNF2-like ATPase domain and a DNA binding domain (DBD) at the C-terminal site (Figure 4A). The tandem chromodomain was dispensable for chromatin binding but especially important for its substrate recognition (Hauk et al., 2010; Morettini et al., 2011). However, indispensable was the DBD domain for fast and directional nucleosome sliding by interaction with the linker DNA of nucleosomes (McKnight et al., 2011; Ryan et al., 2011). Moreover, also mutations in the DBD domain did not prevent CHD1 from chromatin association (McKnight et al., 2011). Surprisingly, substitution of the DBD domain and changed tethering of CHD1 directly onto the nucleosome substrate changed its sliding properties and even caused disruption of the nucleosome (Patel et al., 2013).

\subsubsection{The role of CHD1 in yeast and drosophila}

In S. cerevisiae and D. melanogaster CHD1 is not necessary for their viability, but latter showed impaired development and fertility (McDaniel et al., 2008; Tsukiyama et al., 1999). However, CHD1 deletion in S. cerevisiae or its orthologue histidine-rich protein (hrp3) in Saccharomyces pombe showed changes in nucleosome positioning over the gene body relative to the stable positioned +1 nucleosome (Gkikopoulos et al., 2011; Hennig et al., 2012; Shim et al., 2012). Furthermore, H3K36me3 was reduced and gene bodies were more acetylated after passage of RNA-Pol II. The resulting changes in nucleosome array and open acetylated chromatin structure at the gene body caused cryptic transcription, but did not alter global transcription. 
Consistently, another study showed that H2Bub1, a mark for transcriptional elongation at gene bodies, was reduced after loss of CHD1 and gene bodies had less nucleosome occupancy (Lee et al., 2012a). Moreover, CHD1 mutant strains showed an increased accumulation of RNA-Pol II Serine 5 phosphorylated form at TSS, which characterizes early elongating defects of the RNA-Pol II, probably by an altered nucleosome array (Park et al., 2014). Besides transcription regulation CHD1 was also required for deposition and regular spacing of $\mathrm{H} 3.3$ in the male pronucleus of $D$. melanogaster (Konev et al., 2007).

\subsubsection{Role of CHD1 in higher eukaryotes}

In contrast to $S$. cerevisiae and $D$. melanogaster $\mathrm{CHD} 1$ is required for the embryonic development in mice (Guzman-Ayala et al., 2015). CHD1 deletion caused prenatally lethality between day five and six which indicates its importance in developmental processes. Similar to $S$. cerevisiae, CHD1 is mainly linked to transcriptional processes in higher eukaryotes. It was described to bind to $\mathrm{H} 3 \mathrm{~K} 4 \mathrm{me} 3$ with its tandem chromodomain and interact with the transcription machinery (Figure 4B) (Lin et al., 2011; Sims et al., 2007). In particular, CHD1 is recruited by the mediator complex and probably further stabilized or positioned to its nucleosome substrate by its binding to H3K4me3. However, it had been shown that CHD1 is not associated with H3K27me3 which indicates its association with active, but not bivalent, genes (Gaspar-Maia et al., 2009). Global binding patterns of CHD1 in human and mice revealed high similarities to yeast. Further, genome-wide occupancy analyses uncovered CHD1 binding also at gene bodies, which is supported by previous interaction studies between CHD1 and structure specific recognition protein 1 (SSRP1), a histone chaperone (Kelley et al., 1999; Skene et al., 2014). If CHD1 is also necessary for the maintenance of H3K36me3 and H2Bub1 at the gene body like it was observed in yeast was not investigated yet (Gkikopoulos et al., 2011; Hennig et al., 2012; Shim et al., 2012).

However, a recent study in mouse embryonic fibroblasts showed that overexpression of a CHD1 mutant protein increased the nucleosome turnover at the gene body which indicates its role in ordering the chromatin after RNA-Pol II passage (Skene et al., 2014). Furthermore, it reduced the nucleosome turnover at the TSS of all expressed genes and caused decreased RNA-Pol II Serine 2 phosphorylation levels, a mark for elongating polymerase. This indicates that functional CHD1 was required for the 
RNA-Pol Il to overcome the +1 nucleosome barrier and order the nucleosomes at the gene body after passage of RNA-Pol II.

Next to its transcriptional effects, studies also highlighted a role for CHD1 in the segmentation of hetero- and euchromatin. In D. melanogaster and mice, reduced CHD1 levels caused increased marks of heterochromatin, like HP1alpha and H3K9me2 (Bugga et al., 2013; Gaspar-Maia et al., 2009). CHD1, however, is restricted to open chromatin states, as it was mutually exclusive with the $\mathrm{H} 1$ histone (Lusser et al., 2005).

The role of CHD1 in transcription was also shown to regulate developmental processes and tumorigenesis. A recent study highlighted that CHD1 is required for the maintenance of mouse embryonic stem cells (mESC) and self-renewal capacity (Gaspar-Maia et al., 2009). The same group also observed that endothelial-specific deletion of $\mathrm{CHD} 1$ caused a loss of hematopoietic progenitor cells, however, interestingly, after hematopoietic differentiation CHD1 deletion had no obvious effects on subsequent blood cell development (Koh et al., 2015). This indicates a specificity in certain differentiation processes and demonstrates that CHD1 played a role in stem cell differentiation and maintenance.

Besides developmental processes, CHD1 is frequently mutated or deleted in human prostate cancer (Grasso et al., 2012), although mutations in transcriptional coactivators are less frequently reported in high proliferative cancer cells. Interestingly, recent studies highlighted the interaction of CHD1 with the androgen receptor (AR) and its role in regulating AR-dependent gene transcription (Burkhardt et al., 2013). However, other studies claim that a subtype of androgen-independent aggressive prostate cancer cells carry CHD1 mutations (Huang et al., 2012; Rodrigues et al., 2015). Although, molecular studies particular in cancer models are missing which address the question how CHD1 affects RNA-Pol II transcription and pausing, the studies so far showed that loss of CHD1 might act gene specific and not on global gene expression. 
A

CHD1 domains:

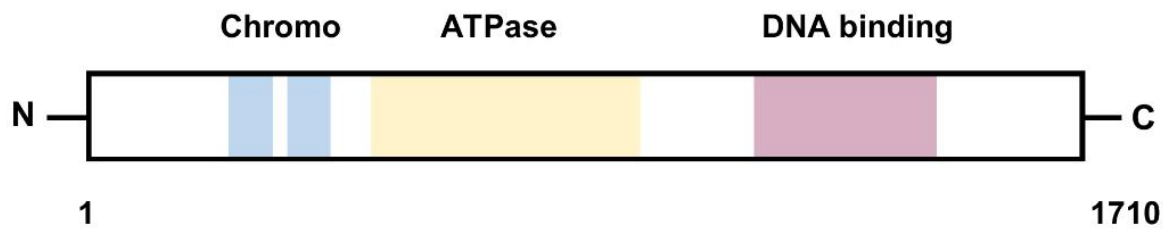

B

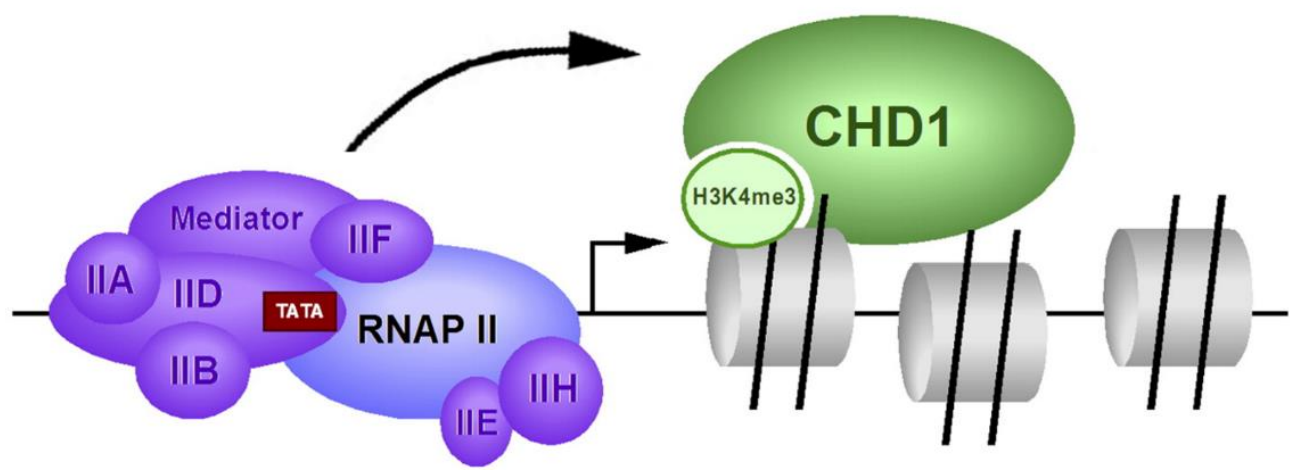

Figure 4:CHD1 domains and its interactions near the transcriptional start site in mammals. A The schematic view of chromodomain helicase DNA-binding protein 1 (CHD1) domains showing the $\mathrm{N}$-terminal tandem chromodomain, SNF2-like ATPase domain and the C-terminal DNA binding domain within the 1710 amino acids long protein. B The model represents CHD1 binding to Histone 3 lysine 4 trimethylation at the transcriptional start site (small arrowhead). The transcription machinery (purple) of the RNA-Polymerase II (RNAP II) is recruited at the nucleosome free region with the TATAbox motif site on the DNA (brown box). CHD1 interacts with the mediator complex (Figure modified after Sims et al., 2007).

\subsection{Stem cells and differentiation}

Stem cells are undifferentiated and unspecialized cell types which have the ability to self-renew throughout their life-span. In general, different classes of stem cells exist which can be distinguished by their differentiation potential (Eckfeldt et al., 2005). Totipotent zygotes have the highest potential and give rise to a complete organism. Moreover, they can differentiate into pluripotent embryonic stem cells (ESC). ESC are characterized by their ability to generate derivatives of the three main germ layers: endoderm, ectoderm and mesoderm. Gradually, each of these three classes can further differentiate and form more committed somatic/adult stem cells (SSC) with the capacity of producing restricted numbers of distinct cells types.

Besides the natural occurring stem cells there exist also induced pluripotent stem cells (iPSC) which were introduced in 2006 and are considered to be a breakthrough 
in the stem cell field (Takahashi and Yamanaka, 2006). It had been shown that mouse somatic cells can be transformed into ESC by only four transcription factors, namely POU class 5 homeobox 1 (POU5F1 or Oct3/4), SRY-box 2 (Sox2), v-myc avian myelocytomatosis viral oncogene homolog (c-Myc), and kruppel-like factor 4 (KLF4). However, the induced pluripotent stem cells (iPSC) partially retained the specific epigenetic memory of the parental somatic cell after reprogramming (Polo et al., 2010).

Although all cells within the hierarchical differentiation program carry the same genotype they vary greatly in function and morphology. To accomplish this the accessibility of the DNA in different cell types is altered by chromatin packaging, DNA methylation or regulatory RNAs which together is termed as epigenetic regulation (Jaenisch and Bird, 2003; Li and Reinberg, 2011). Already in 1957 Conrad Waddington introduced a model which describes the role of the epigenetic landscape underlying the stem cell differentiation and cell fate determination (Figure 5A) (Waddington, 1957). As depicted by Waddington, the epigenetic regulation with its various possible modifications in the genome has a great influence on the cell fate determination and regulation.

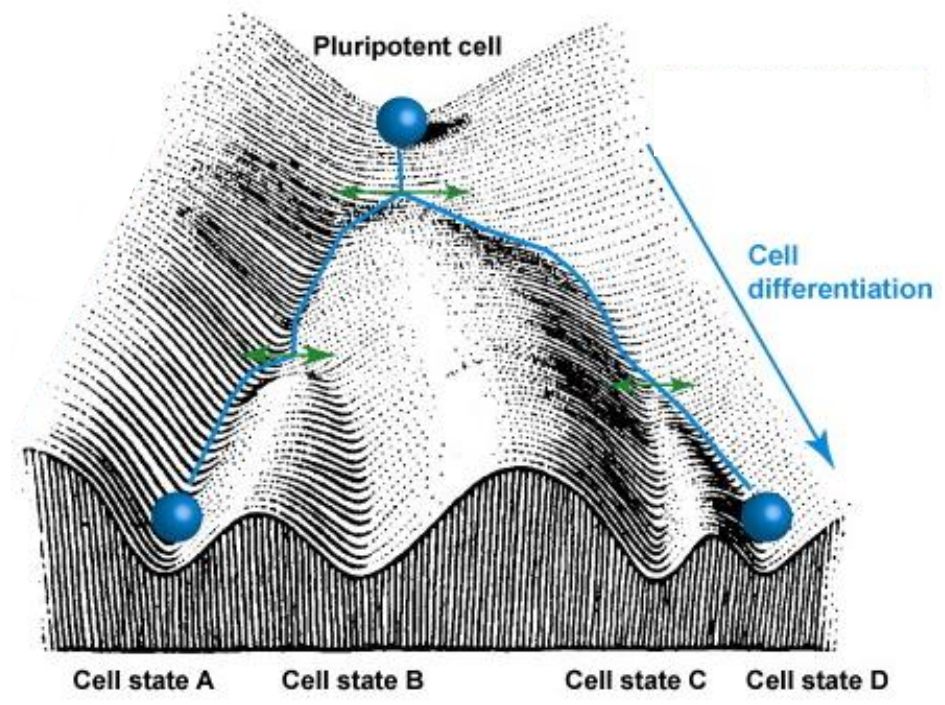

Figure 5: Epigenetic landscape model by Waddington. An undifferentiated pluripotent stem cell (blue ball) on the top of the landscape with a high differentiation potential can differentiate into more committed cell states "downhill". Each event of cellular commitment will be accompanied by epigenetic changes that are depicted here as valleys which greatly influences the final outcome of the differentiated cell state (Figure modified after Barth and Imhof, 2010). 


\subsection{MSC and their differentiation potential}

MSC are somatic multipotent stem cells which reside in pre- and postnatal tissues like muscle, fat, bone or cartilage (Owen and Friedenstein, 1988). Recent critical rethinking however questioned that there is only one general MSC cell type (MéndezFerrer et al., 2010; Zhou et al., 2014). Briefly, it is hypothesized that different types of MSC exist which vary in their internally determined cell fates, defined by their progenitors and tissue they are residing in (Kassem and Bianco, 2015). For example, adipose tissue derived MSCs are reprogrammable to osteoblasts but show neither spontaneous nor in vivo capacity to form bone, whereas bone marrow derived MSC do. Furthermore, different MSC can vary in their responses to differentiation stimuli in their outcome of cell states (Al-Nbaheen et al., 2012).

Bone marrow derived MSC (or skeletal stem cells) give rise to different tissues that are present in the skeleton such as bone, cartilage and fat. MSC reside perivascular within a stem cell niche inside the bone marrow together with hematopoietic stem cells (HSC) where they can mutually regulate each other's differentiation (MéndezFerrer et al., 2010). In addition, the strong vascular and microvascular environment of the bones influence the interplay between the hematopoietic and mesenchymal cell systems (Bianco and Robey, 2015). This shows that both systems are closely coupled and interact with each other in a paracrine manner. MSC express several receptor types such as insulin growth factor 1 receptor (IGF1R), transforming growth factor receptors (TGFR) or bone morphogenic protein receptors (BMPR) by which different signaling pathways can be triggered and thereby induce cell fate shifts with transcriptional and epigenetic changes.

\subsubsection{Adipocyte differentiation}

MSC differentiation to adipocytes is a postnatal event usually occurring with aging, however, it is also enhanced in diseased bone with low bone mass or in osteoporosis (Abdallah and Kassem, 2012; Rosen and Bouxsein, 2006). Different studies showed that a more adipocyte or osteoblast precursor cell type can favor the cell lineage differentiation which might be shifted with age or disease (Post et al., 2008; Russell et al., 2010). Adipocyte differentiation is mainly driven by the nuclear hormone receptor peroxisome proliferator-activated receptor gamma (PPARG), which is required for early adipocyte differentiation and maintenance of the adipocyte cell type (Rosen and MacDougald, 2006). Ligands which bind PPARG are still elusive, but 
fatty acids and their metabolites or synthetically derivatives of glitazones such as troglitazone were shown to activate PPARG (Ahmadian et al., 2013; Mukherjee et al., 1997). Upon activation, PPARG forms a heterodimer with the retinoid $X$ receptor $(R X R)$ and regulates gene expression by interaction with $C R C$ and co-activators like p300 (Gelman et al., 1999; Salma et al., 2004). Moreover, the transcription factors transcriptional CCAAT/enhancer-binding protein a (C/EBPa) and different KLFs play an important role in early and late stages of adipocyte differentiation (Mori et al., 2005; Oishi et al., 2005; Tang et al., 2003).

\subsubsection{Osteoblast differentiation}

The bone is a dynamic organ and undergoes life-long remodeling by continuous deposition and absorption of mineralized tissue (Clarke, 2008). In this process the role of osteoblast differentiation is indispensable to maintain the balance between bone formation and resorption. In general, osteoblast differentiation can be divided into early and late stages that involve progenitor cells, pre-osteoblasts and mature osteoblast formation (Long, 2012). During these stages the cells first undergo a high proliferation with subsequent deposition of an organic extracellular-scaffold which is finally mineralized in mature osteoblasts (Lian and Stein, 1992). The early cell lineage commitment happens under the influence of TGF-beta or BMP signaling (Chen et al., 2012).

The osteoblast progenitor cells and early differentiating osteoblasts are mainly determined by the transcription factor runt-related transcription factor 2 (RUNX2) which is activated by different signaling molecules such as TGF-beta and BMP-2 (Lee et al., 2000, 2003). However, unlike PPARG RUNX2 is not required for the maintenance of osteoblastic cell fate and even needs to be inactivated in mature osteoblasts (Komori, 2009).

Another important regulator of osteoblast differentiation is the canonical and noncanonical Wnt signaling pathway (Rodda and McMahon, 2006; Taipaleenmäki et al., 2011). Striking evidence for the role of the canonical pathway for osteogenesis was discovered by studies which showed that removal of $\beta$-catenin in MSC progenitor cells reduced osteoblast differentiation (Day et al., 2005; Hu et al., 2005). Interestingly, the repression of osteoblast differentiation was induced by the loss of the $\beta$-catenin and favored instead chondrogenic differentiation of the MSCs (Hill et 
al., 2005; Rodda and McMahon, 2006). Although, the early osteoblast differentiation marker RUNX2 was still expressed in the surrounding tissue, Sp7 transcription factor (SP7), a marker for later osteoblast differentiation stages, was not.

The multifactorial Wnt signaling plays, however, a more complicated role in osteoblast differentiation by acting either as an activator or suppressor of differentiation which depends on the cell lineage commitment (Regard et al., 2012). Canonical Wnt activation in MSC causes increased proliferation but decreased differentiation (de Boer et al., 2004; Boland et al., 2004; Regard et al., 2012), whereas in pre-osteoblasts or early committed cells Wnt signaling enhances differentiation and growth (Eijken et al., 2008; Rodda and McMahon, 2006). Furthermore, the terminal differentiation can be blocked by active Wnt signaling (Kahler et al., 2006, 2008). This indicates a dynamic pattern of gene expression and complex regulation during osteoblast differentiation.

Concordant with these findings showed recent genome-wide transcriptome studies that the factors involved in osteogenesis can fluctuate in their expression levels during differentiation (Kulterer et al., 2007; Twine et al., 2014). One conclusion of the authors was that the temporal fine tuning in gene expression of osteoblastassociated factors could play pivotal roles for an ordered osteoblast differentiation. This is in line with the different impact of Wnt factors or RUNX2 which are pivotal at certain stages of differentiation but can be repressive at other stages during osteogenesis (Kahler and Westendorf, 2003; Kahler et al., 2006; Kanatani et al., 2006)

\subsubsection{MSC in clinical studies}

MSCs are one of the most often used cell types for stem cell therapy in clinical approaches (Wei et al., 2013). When comparing the registered clinical trials for MSC in the United States between December 2012 and December 2015 the number doubled from 281 to 565 (ClinicalTrials.gov). For stem cell therapy living cells are either systemically or locally applied so that they home to degenerated or injured tissue to restore its function or to support its regeneration. MSCs have the advantages that they are easy to isolate from different tissues within the body and can be expanded ex vivo (Zaher et al., 2014). Moreover, they have immunomodulatory properties which lower the danger of immune-rejection by the 
host system (Atoui and Chiu, 2012). Although their division number ex vivo is limited, as they undergo replicative senescence, ways of immortalization by telomerase reverse transcriptase (TERT) were described without impairment of their differentiation potential (Simonsen et al., 2002). Nonetheless providing efficient ex vivo expansion without changing the cellular properties remain a concern in stem cell therapies (Hoch and Leach, 2014)

Preclinical studies of stem cell therapies showed that MSCs were already successfully used for tissue regeneration processes in liver, lung or bone (Amado et al., 2005; Kim et al., 2007; Lee et al., 2009; Parekkadan et al., 2007; Tzaribachev et al., 2008). In particular for the bone tissue repair of defective cartilage, treatment of patients with osteogenesis imperfect or bone fracture healing were reported (GómezBarrena et al., 2015; Le Blanc et al., 2005; Wakitani et al., 2007). Regenerative properties of MSC are either provided by direct differentiation into bone tissue within an organic scaffold implanted during surgery or by their paracrine signaling of cytokines inhibiting apoptosis and support angiogenesis (Rosset et al., 2014; Wei et al., 2013). Interestingly, co-transplantation of MSC and HSC increased tissue repair effects in a primate animal models which indicates a potential synergism between the two stem cell systems (Chapel et al., 2003).

Moreover MSC might be also suitable for treatment of osteoporosis (Antebi et al., 2014). Osteoporosis is a disease defined by porous and fragile bone caused mainly by an imbalanced system between bone formation and bone resorption (Rachner et al., 2011). Most of the actual approved therapies are antiresorptive approaches, however, enhancing the bone formation shows alternative ways of therapy which are likely to reduce negative side effects (Chen et al., 2014a). MSC cell therapy could support the bone building process by osteoblast differentiation at the porous sites in vivo (Antebi et al., 2014). 


\subsection{Aim of the study}

The chromatin remodeler CHD1 is a well described co-activator of gene transcription and is associated with various transcription associated complexes. However, so far it is less clear if CHD1 can also act as a regulatory protein in gene expression and if it is required for gene induction. Here we hypothesize that CHD1 is necessary for gene specific expression during adipocyte and osteoblast differentiation and is required for the cell type change. To unravel the molecular mechanism of its gene regulation we performed genome-wide ChIP-seq and RNA-seq in control and CHD1 depleted condition. We focused on changes of the transcriptional hallmarks RNA-PoI II, H2A.Z and H2Bub1 of differentiation and CHD1 depletion regulated genes. Besides the mechanistic effect the biological impact on bone formation was investigated. Therefore, transcriptome wide analysis of deregulated osteoblast-specific differentiation genes was performed. Additionally, ectopic bone formation capacity of stable CHD1 depleted MSC was tested to test the role of $\mathrm{CHD1}$ in vivo. 


\section{Material}

\subsection{Technical equipment}

$-150{ }^{\circ} \mathrm{C}$ Freezer (MDF-C2156VAN)

$-20{ }^{\circ} \mathrm{C}$ Freezer

2100 Bioanalyzer

$-80{ }^{\circ} \mathrm{C}$ Freezer "Hera freeze"

Agarose gel chamber

\section{Balance}

Bandelin Sonoplus Sonicator

Biological Safety Cabinet "Safe 2020"

Bioruptor $^{\circledR}$ Plus sonication device

Centrifuge (Megafuge 1.OR)

Centrifuge $4{ }^{\circ} \mathrm{C}$ (5417R)

Centrifuge $4{ }^{\circ} \mathrm{C}$ (Fesco 21)

Counting chamber (Neubauer)

DynaMag $^{\mathrm{TM}} 2$

DynaMag $^{\text {TM }} 96$ Side

Eclipse TS100

Electrophoresis \& Electrotransfer Unit

Gel iX Imager

HERAcell 150i $\mathrm{CO}_{2}$ Incubator

Imager Western Blot

Inverse Microscope "Axiovert 40 CFL"

Isotemp ${ }^{\circledR}$ water bath

Magnet stirrer "MR3001"

Microcentrifuge C1413-VWR230

Microwave

Mini Trans-Blot ${ }^{\mathrm{TM}}$ Cell

Mini-PROTEAN Tetra Cell

Mr. Frosty ${ }^{\circledR}$ Cryo Freezing Container

Nano Drop ${ }^{\circledR}$ ND-1000

Optical Reaction Module CFX96 ${ }^{\mathrm{TM}}$

pH meter inoLab ${ }^{\circledR}$
Panasonic, Kadoma, Japan

Liebherr $\mathrm{GmbH}$, Biberach

Agilent Technology, Santa Clara, USA

Thermo Fisher Scientific, Waltham, USA

Harnischmacher Labortechnik, Kassel

Sartorius AG, Göttingen

Bandelin electr. $\mathrm{GmbH} \& \mathrm{Co}$. KG, Berlin

Thermo Fisher Scientific, Waltham, USA

Diagenode SA, Liège, Belgium

Thermo Fisher Scientific, Waltham, USA

Eppendorf AG, Hamburg

Thermo Fisher Scientific, Waltham, USA

Brand $\mathrm{GmbH} \&$ Co. KG, Wertheim

LifeTechnology, Carlsbad, USA

LifeTechnology, Carlsbad, USA

Nikon, Tokio, Japan

GE Healthcare Europe $\mathrm{GmbH}$, München Intas Science Imaging $\mathrm{GmbH}$, Göttingen Thermo Fisher Scientific, Waltham, USA Bio-Rad Laboratories, Hercules, USA

Carl Zeiss Microlmaging $\mathrm{GmbH}$, Göttingen Thermo Fisher Scientific, Waltham, USA Heidolph GmbH \& Co. KG, Schwabach VWR, Radnor, USA

Clatronic International $\mathrm{GmbH}$, Kempen Bio-Rad Laboratories, Hercules, USA Bio-Rad Laboratories, Hercules, USA Thermo Fisher Scientific, Waltham, USA Peqlab Biotechnology $\mathrm{GmbH}$, Erlangen Bio-Rad Laboratories, Hercules, USA WTW GmbH, Weilheim 
Pipette Aid ${ }^{\circledR}$ portable XP

Pipettes "Research" Series

Power supply Power Pack P25T

PowerPac ${ }^{\mathrm{TM}}$ Basic Power Supply

PowerPac ${ }^{\mathrm{TM}} \mathrm{HC}$ Power Supply

Refrigerator

Repeat Pipette

Scanner Epson V700 Photo

Shaker "Rocky"

Test tube rotator

Thermal Cycler T100 ${ }^{\mathrm{TM}}$

Thermo mixer $\mathrm{C}$

Vortex-Genie 2

$\mathrm{X}$-Ray Cassettes
Drummond Scientific Co., Broomall, USA

Eppendorf AG, Hamburg

Biometra $\mathrm{GmbH}$, Göttingen

Bio-Rad Laboratories, Hercules, USA

Bio-Rad Laboratories, Hercules, USA

Liebherr $\mathrm{GmbH}$, Biberach

Gilson Inc., Middleton, USA

Seiko Epson, Suwa, Japan

Schütt Labortechnik GmbH, Göttingen

Schütt Labortechnik $\mathrm{GmbH}$, Göttingen

Bio-Rad Laboratories, Hercules, USA

Eppendorf AG, Wessling-Berzdorf

Electro Scietific Industr. Inc., Portland, USA

Rego X-ray GmbH, Augsburg

\subsection{Consumable materials}

96-well Multiplate ${ }^{\circledR}$ PCR plate white

Cell scraper $(16 \mathrm{~cm})$

Cellstar 6- and 12-well cell culture plate Greiner Bio-One $\mathrm{GmbH}$, Frickenhausen

Cellstar PP-tube 15 and $50 \mathrm{ml}$

Cellstar tissue culture dish 100×20 mm Greiner Bio-One $\mathrm{GmbH}$, Frickenhausen

Cellstar tissue culture dish $145 \times 20$ mm Greiner Bio-One $\mathrm{GmbH}$, Frickenhausen

Cryo Tube Vial $(1.8 \mathrm{ml})$

DNA loBinding Tube 1.5 and $0.5 \mathrm{ml}$

Gel blotting paper (Whatman paper)

Hybond $^{\mathrm{TM}}$-PVDF Transfer Membrane

Microtube $1.5 \mathrm{ml}$

Microtube $1.5 \mathrm{ml}$, conical

Microtube $2 \mathrm{ml}$

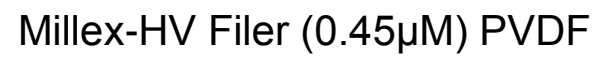

Parafilm ${ }^{\circledR}$ "M"

Pipette filter tips

Pipette tips

X-ray films "Super RX"
Bio-Rad Laboratories, Hercules, USA

Sarstedt AG \& Co., Nümbrecht
Eppendorf AG, Wessling-Berzdorf

Sartorius AG, Göttingen

GE Healthcare Europe $\mathrm{GmbH}$, München

Sarstedt AG \& Co., Nümbrecht

VWR International GmbH, Darmstadt

Sarstedt AG \& Co., Nümbrecht

Merck Millipore KGaA, Darmstadt

Pechiney Plastic Packaging, Chicago, USA

Sarstedt AG \& Co., Nümbrecht

Greiner Bio-One $\mathrm{GmbH}$, Frickenhausen

Fujifilm Corp., Tokyo, Japan 


\subsection{Chemicals}

Acetic acid

Adefodur WB developing concentrate

Adefodur WB fixing concentrate

Adenosin triphosphate

Agarose

Agencourt ${ }^{\circledR}$ AMPure ${ }^{\circledR}$ XP Beads

Albumin Fraction $\mathrm{V}$

Ammonium persulfate

Ammonium sulfate

Ampicilin

Anti-Anti

Aprotinin

Bromophenol blue

Calcitriol

Calcium Chloride

Chloroform

Dexamethasone

Diethylpyrocarbonate

dihydrate

Dimethyl sulfoxide

di-Sodium hydrogen phosphate -

Dithiothreitol

DMEM

DMEM/F12

dNTP

Ethanol absolute

Ethidium bromide

Ethylenediaminetetraacetic acid

Fetal Bovine Serum

Formaldehyde

Glycerol
Carl Roth GmbH \& Co. KG, Karlsruhe

Adefo-Chemie GmbH, Dietzenbach

Adefo-Chemie $\mathrm{GmbH}$, Dietzenbach

Fermentas $\mathrm{GmbH}$, St. Leon-Rot

Carl Roth $\mathrm{GmbH}$ \& Co. KG, Karlsruhe

Beckman Coulter Inc. Brea USA

Carl Roth GmbH \& Co. KG, Karlsruhe

Carl Roth GmbH \& Co. KG, Karlsruhe

Carl Roth GmbH \& Co. KG, Karlsruhe

AppliChem $\mathrm{GmbH}$, Darmstadt

LifeTechnology, Carlsbad, USA

Carl Roth GmbH \& Co. KG, Karlsruhe

Sigma-Aldrich Co., St. Louis, USA

Biomol GmbH, Hamburg

Carl Roth GmbH \& Co. KG, Karlsruhe

Carl Roth GmbH \& Co. KG, Karlsruhe

Sigma-Aldrich Co, St. Louis, USA

Carl Roth GmbH \& Co. KG, Karlsruhe

AppliChem $\mathrm{GmbH}$, Darmstadt

Carl Roth GmbH \& Co. KG, Karlsruhe

Carl Roth GmbH \& Co. KG, Karlsruhe

LifeTechnology, Carlsbad, USA

Life Technology, Carlsbad, USA

Promega $\mathrm{GmbH}$, Mannheim

Th. Geyer GmbH \& Co. KG, Renningen

Carl Roth GmbH \& Co. KG, Karlsruhe

Carl Roth $\mathrm{GmbH} \&$ Co. KG, Karlsruhe

Thermo Scientific HyClone, Logan, USA

Sigma-Aldrich Co., St. Louis, USA

Carl Roth GmbH \& Co. KG, Karlsruhe 
Glycine

Hydrochloric acid

Immobilon ${ }^{\mathrm{TM}}$ Western HRP substrate

Insulin

lodoacetamide

Isopropanol

L-Ascorbic acid

Leupeptin

Linear Acrylamide

Magnesium chloride

MEM a powder

Methanol

Monopotassium phosphate

$\mathrm{N}, \mathrm{N}$-Dimethylformamide

$\mathrm{N}$-ethylmaleimide

Nonidet $^{\mathrm{TM}}$ P40

Oil Red O

Opti-MEM

PBS tablets

Pefabloc SC

Penicillin-Streptomycin solution

Peptone

Polybrene

Potassium acetate

Potassium chloride

Potassium dihydrogen phosphate

Propidium iodide solution

Protein A Sepharose ${ }^{\mathrm{TM}} \mathrm{CL}-4 \mathrm{~B}$

RNAIMAX

Roti ${ }^{\circledR}$-Phenol

Rotiphorese ${ }^{\circledR}$ Gel 30

Rotipuran ${ }^{\circledR}$ Chloroform

Rotipuran ${ }^{\circledR}$ Isoamylalcohol

Sepharose $^{\mathrm{TM}} \mathrm{CL}-4 \mathrm{~B}$
Carl Roth $\mathrm{GmbH} \&$ Co. KG, Karlsruhe Carl Roth $\mathrm{GmbH} \&$ Co. KG, Karlsruhe Merck Millipore KGaA, Darmstadt Sigma-Aldrich Co, St. Louis, USA Sigma-Aldrich Co, St. Louis, USA Carl Roth $\mathrm{GmbH} \&$ Co. KG, Karlsruhe Sigma-Aldrich Co, St. Louis, USA Carl Roth $\mathrm{GmbH} \&$ Co. KG, Karlsruhe Thermo Fisher Scientific, Waltham, USA Carl Roth $\mathrm{GmbH} \&$ Co. KG, Karlsruhe LifeTechnologies AG, Carlsbad, US Carl Roth $\mathrm{GmbH} \&$ Co. KG, Karlsruhe Carl Roth $\mathrm{GmbH} \&$ Co. KG, Karlsruhe Sigma-Aldrich Co., St. Louis, USA Sigma-Aldrich Co., St. Louis, USA Sigma-Aldrich Co., St. Louis, USA Sigma-Aldrich Co., St. Louis, USA LifeTechnology, Carlsbad, USA LifeTechnology, Carlsbad, USA Carl Roth $\mathrm{GmbH}$ \& Co. KG, Karlsruhe Sigma-Aldrich Co., St. Louis, USA Carl Roth $\mathrm{GmbH} \&$ Co. KG, Karlsruhe Sigma-Aldrich, St. Louis, USA Carl Roth $\mathrm{GmbH}$ \& Co. KG, Karlsruhe AppliChem $\mathrm{GmbH}$, Darmstadt Carl Roth $\mathrm{GmbH}$ \& Co. KG, Karlsruhe Sigma-Aldrich Co., St. Louis, USA GE Healthcare, Uppsala, Sweden LifeTechnology, Carlsbad, USA Carl Roth $\mathrm{GmbH} \&$ Co. KG, Karlsruhe Carl Roth $\mathrm{GmbH} \&$ Co. KG, Karlsruhe Carl Roth $\mathrm{GmbH} \&$ Co. KG, Karlsruhe Carl Roth $\mathrm{GmbH} \&$ Co. KG, Karlsruhe GE Healthcare, Uppsala, Sweden 
Skim milk powder

Sodium acetate

Sodium chloride

Sodium deoxycholate

Sodium dodecylsulfate

Sodium hydroxide

B-Glycerolphosphate

SYBR Green

TEMED

Tris

Triton X-100

Trypsin-EDTA

Tween-20

a, $\alpha$-Trehalose Dihydrate
Carl Roth GmbH \& Co. KG, Karlsruhe Carl Roth $\mathrm{GmbH} \&$ Co. KG, Karlsruhe Carl Roth GmbH \& Co. KG, Karlsruhe AppliChem $\mathrm{GmbH}$, Darmstadt Carl Roth GmbH \& Co. KG, Karlsruhe Carl Roth $\mathrm{GmbH} \&$ Co. KG, Karlsruhe Sigma-Aldrich Co., St. Louis, USA Roche Diagnostics $\mathrm{GmbH}$, Mannheim Carl Roth $\mathrm{GmbH}$ \& Co. KG, Karlsruhe Carl Roth $\mathrm{GmbH} \&$ Co. KG, Karlsruhe AppliChem $\mathrm{GmbH}$, Darmstadt LifeTechnology, Carlsbad, USA AppliChem $\mathrm{GmbH}$, Darmstadt Panreac AppliChem GmbH, Darmstadt

\subsection{Kits and reagents}

Agilent High Sensitivity DNA Kit Agilent RNA 6000 Nano Kit Lipofectamine $^{\mathrm{TM}} 2000$ Lipofectamine $^{\text {TM }}$ RNAiMAX MicroPlex Library Preparation ${ }^{\mathrm{TM}}$ NEBNext ${ }^{\circledR}$ Poly(A) mRNA Module NEBNext ${ }^{\circledR}$ Ultra $^{\text {TM }}$ Library Prep Kit Qubit dsDNA HS Assay
Agilent Technology, Santa Clara, USA Agilent Technology, Santa Clara, USA LifeTechnology, Carlsbad, USA LifeTechnology, Carlsbad, USA Diagenode SA, Liège, Belgium New England Biolabs, Ipswich, USA New England Biolabs, Ipswich, USA LifeTechnology, Carlsbad, USA 


\subsection{Nucleic acids}

\subsubsection{Vectors for viral particle production}

\begin{tabular}{|c|c|c|}
\hline Construct name & shRNA-sequence & Source \\
\hline $\begin{array}{c}\text { GIPZ shRNA-CHD1 \#1 } \\
\text { Clone V3THS_312675 }\end{array}$ & TTTTTGTAAGAATCTCCCT & $\begin{array}{c}\text { Dharmacon Inc (Lafayette, } \\
\text { USA) }\end{array}$ \\
\hline $\begin{array}{c}\text { GIPZ shRNA-CHD1 \#2 } \\
\text { Clone V2THS_112971 }\end{array}$ & ATAATTCAGAAATGAGATC & $\begin{array}{c}\text { Dharmacon Inc (Lafayette, } \\
\text { USA) }\end{array}$ \\
\hline $\begin{array}{c}\text { GIPZ non-targeting- } \\
\text { control }\end{array}$ & TACTCTCGCCCAAGCGAG & $\begin{array}{c}\text { Dharmacon Inc (Lafayette, } \\
\text { USA) }\end{array}$ \\
\hline $\begin{array}{c}\text { psPAX2 packaging } \\
\text { vector }\end{array}$ & - & V. Assmann UKE, Hamburg \\
\hline $\begin{array}{c}\text { pMD2.G envelope } \\
\text { vector }\end{array}$ & - & V. Assmann UKE, Hamburg \\
\hline
\end{tabular}

\subsubsection{Oligonucleotides}

\subsubsection{1 siRNA oligonucleotides}

\begin{tabular}{|c|c|c|c|}
\hline Name & $\begin{array}{c}\text { Target } \\
\text { Gene }\end{array}$ & siRNA Target Sequence & Source \\
\hline siCHD1-1 & CHD1 & CAUCAAGCCUCAUCUAAUA & $\begin{array}{c}\text { Dharmacon Inc } \\
\text { (Lafayette, } \\
\text { USA) }\end{array}$ \\
\hline siCHD1-2 & CHD1 & GAUAAGAACUCAUGAAUGG & $\begin{array}{c}\text { Dharmacon Inc } \\
\text { (Lafayette, } \\
\text { USA) }\end{array}$ \\
\hline siCHD1-3 & CHD1 & GAAGAGAGCUGAAACUCAU & $\begin{array}{c}\text { Dharmacon Inc } \\
\text { (Lafayette, } \\
\text { USA) }\end{array}$ \\
\hline $\begin{array}{c}\text { siCHD1-4 } \\
\text { CHD1 }\end{array}$ & GAAACAAGCUCUAGAUCAU & $\begin{array}{c}\text { Dharmacon Inc } \\
\text { (Lafayette, } \\
\text { USA) }\end{array}$ \\
\hline $\begin{array}{c}\text { Luciferase GL2 } \\
\text { duplex }\end{array}$ & CGUACGCGGAAUACUUCGA & $\begin{array}{c}\text { Dharmacon Inc } \\
\text { (Lafayette, } \\
\text { USA) }\end{array}$ \\
\hline
\end{tabular}

For siCHD1 transfection reactions the individual siRNAs directed against CHD1 were pooled in a $1: 1: 1: 1$ ratio.

\subsubsection{RT-PCR primers}

For reverse transcription reaction random 9mer primer were used. The primers were 
purchased from Sigma-Aldrich Co., St. Louis, USA

\subsubsection{3 qPCR primers}

Primers are shown in a $5^{\prime}$ to $3^{\prime}$ orientation. Own primers were designing by the program primer -blast available at NCBI (www.ncbi.nlm.nih.gov/tools/primer-blast/).

\begin{tabular}{|l|l|l|}
\hline Name & Sequence & Source \\
\hline 18S rRNA F & AACTGAGGCCATGATTAA & Nagarajan et al. 2015 \\
\hline 18S rRNA R & GGAACTACGACGGTATCTGA & Nagarajan et al. 2015 \\
\hline ALPL F & TGGGCCAAGGACGCTGGGAA & Karpiuk et al. 2012 \\
\hline ALPL R & AAGGCCTCAGGGGGCATCTCG & Karpiuk et al. 2012 \\
\hline AXIN2 F & ATTTCCCGAGAACCACCGCCT & This study \\
\hline AXIN2 R & GGCTGTGGCGGCTCTCCAAC & This study \\
\hline BGLAP F & CACTCCTCGCCCTATTGGC & Karpiuk et al. 2012 \\
\hline BGLAP R & CTTGGACACAAAGGCTGCAC & Karpiuk et al. 2012 \\
\hline BMP4 F & GGAGCTTCCACCACGAAGAA & This study \\
\hline BMP4 R & GGAAGCCCCTTTCCCAATCA & This study \\
\hline CHD1 F & GCCAAGGTTTGTAGCCCTGA & This study \\
\hline CHD1 R & GCACCAAGAATGAGCAAGCC & This study \\
\hline COL11A1 F & CTCAGGGACCTGCAGGAAAG & This study \\
\hline COL11A1 R & GGGTCACCTTTGAGACCAGG & This study \\
\hline CTGF F & CACCCGGGTTACCAATGACA & This study \\
\hline CTGF R & GGATGCACTTTTTGCCCTTCTTA & This study \\
\hline DUSP1 F & GAGCTGTGCAGCAAACAGTC & This study \\
\hline DUSP1 R & GGGCCACCCTGATCGTAGA & This study \\
\hline EDN1 F & TTGAGATCTGAGGAACCCAC & This study \\
\hline EDN1 R & CAGCGCCTAAGACTGCTGTT & This study \\
\hline ELN F & TCCCGGGAGTTGGCATTTC & This study \\
\hline ELN R & ACTGGGCGGCTTTGGC & This study \\
\hline LPIN1 F & CCGCTCGGTGCAGACCAT & This study \\
\hline LPIN1 R & GGACCCCCATCTTCCCAAAG & This study \\
\hline LPL F & TCAGCCGGCTCATCAGTCGGT & Karpiuk et al. 2012 \\
\hline LPL R & AGAGTCAGCACGAGCAGGGCT & Karpiuk et al. 2012 \\
\hline PDK4 F & AGAGGTGGAGCATTTCTCGC & Karpiuk et al. 2012 \\
\hline PDK4 R & ATGTTGGCGAGTCTCACAGG & Karpiuk et al. 2012 \\
\hline PLIN1 F & ACCTCCTCCCTCCAGACAAG & This study \\
\hline PLIN1 R & ATGGTCTGCACGGTGTATCG & This study \\
\hline POSTN F & TTCATTGAAGGTGGTGATGGTCA & This study \\
\hline POSTN R & CTTGCAACTTCCTCACGGGT & This study \\
\hline PPARG F & ACCTCCGGGCCCTGGCAAAA & Karpiuk et al. 2012 \\
\hline PPARG R & TGCTCTGCTCCTGCAGGGGG & Karpiuk et al. 2012 \\
\hline RPLP0 F & GATTGGCTACCCAACTGTTG & Fritah et al., 2005 \\
\hline RPLP0 R & CAGGGGCAGCAGCCACAAA & Fritah et al., 2005 \\
\hline
\end{tabular}




\begin{tabular}{|l|l|l|} 
SIX1 $\mathrm{F}$ & AAGAACCGGAGGCAAAGAGAC & This study \\
\hline SIX1 R & AAGGACCGAGTTCTGGTCTG & This study \\
\hline
\end{tabular}

\subsubsection{ChIP primers}

Primers utilized in ChIP qPCR are shown in $5^{\prime}$ to $3^{\prime}$ orientation.

\begin{tabular}{|l|l|l|}
\hline Name & Sequence & Source \\
\hline DUSP1 TSS F & CTGAAGCGAGGTTGACAGA & This study \\
\hline DUSP1 TSS R & GGCCATGGTCATGGAAGTGG & This study \\
\hline GAPDH TSS F & AAGAAGATGCGGCTGACTGT & Nagarajan et al., 2015 \\
\hline GAPDH TSS R & CGGCTACTAGCGGTTTTACG & Nagarajan et al., 2015 \\
\hline RPLP0 TSS F & CAATCAGAAACCGCGGATAG & Nagarajan et al., 2015 \\
\hline RPLP0 TSS R & CTTCGCGACCCTACTTAAAGG & Nagarajan et al., 2015 \\
\hline TFF TSS F & ACACCCACCTTCCACAACAC & Nagarajan et al., 2015 \\
\hline TFF TSS R & CAGGCTTCTCCCTTGATGA & Nagarajan et al., 2015 \\
\hline
\end{tabular}

\subsection{Proteins, enzymes, standards}

\subsubsection{Molecular weight standards}

Gene Ruler ${ }^{\mathrm{TM}}$ DNA-Ladder

PageRuler ${ }^{\mathrm{TM}}$ Prestained Protein Ladder

\subsubsection{Enzymes}

Proteinase $\mathrm{K}$

Reverse Transcriptase (M-MuLV)

RNase A

RNase inhibitor

T4 DNA Ligase

Taq DNA Polymerase
Fermentas $\mathrm{GmbH}$, St. Leon-Rot

Fermentas $\mathrm{GmbH}$, St. Leon-Rot

LifeTechnology, Carlsbad, USA

New England Biolabs, FFM

Qiagen $\mathrm{GmbH}$, Hilden

New England Biolabs, FFM

New England Biolabs, FFM

Prime Tech, Minsk, Belarus 


\subsubsection{Antibodies}

\subsubsection{Primary antibodies}

Antibodies used for ChIP and Western blot analysis and the respective dilutions

\begin{tabular}{|c|c|c|c|c|}
\hline Target Protein & Cat. No $^{\circ}$ & ChIP & WB & Source \\
\hline CHD1 & A301-218A & $1.5 \mu \mathrm{g}$ & - & $\begin{array}{c}\text { Bethyl Laboratories, Inc., } \\
\text { Montgomery, USA }\end{array}$ \\
\hline CHD1 & sc-271636 & - & $1: 10,000$ & $\begin{array}{c}\text { Santa Cruz Biotech., Inc., Texas } \\
\text { USA }\end{array}$ \\
\hline H2A.Z & - & $5 \mu \mathrm{l}$ & - & AG Gaudreau \\
\hline H2B & $07-371$ & - & $1: 10,000$ & $\begin{array}{c}\text { Merck Millipore, KGaA, } \\
\text { Darmstadt }\end{array}$ \\
\hline H2Bub1 & 5546 & $1.5 \mu \mathrm{g}$ & - & $\begin{array}{c}\text { Cell Signaling, Inc., Danvers } \\
\text { USA }\end{array}$ \\
\hline H2Bub1 & - & - & $1: 50$ & \begin{tabular}{c} 
Prenzel et al., 2011 \\
\hline HSC70
\end{tabular} \\
\hline sc-7298 & - & $1: 25,000$ & $\begin{array}{c}\text { Santa Cruz Biotech., Inc., Texas } \\
\text { USA }\end{array}$ \\
\hline $\begin{array}{c}\text { IgG (non- } \\
\text { specific) }\end{array}$ & ab46540 & $1 \mu \mathrm{g}$ & - & Abcam, Cambridge, UK \\
\hline RNA-PollI & sc-899 & $1.5 \mu \mathrm{g}$ & $1: 10,000$ & $\begin{array}{c}\text { Santa Cruz Biotech., Inc., Texas } \\
\text { USA }\end{array}$ \\
\hline$\beta$ B-Actin & ab6276 & - & $1: 10,000$ & Abcam, Cambridge, UK \\
\hline
\end{tabular}

The antibody dilutions for the immuno based analysis were supplemented with $0.01 \%$ sodium azide.

\subsubsection{Secondary antibodies}

\begin{tabular}{|l|l|l|}
\hline Name & WB & Source \\
\hline anti-mouse (IgG)-HRP & $1: 10,000$ & $\begin{array}{l}\text { Santa Cruz Biotech., Inc., } \\
\text { Texas USA }\end{array}$ \\
\hline anti-rabbit (IgG)-HRP & $1: 10,000$ & $\begin{array}{l}\text { Santa Cruz Biotech., Inc., } \\
\text { Texas USA }\end{array}$ \\
\hline
\end{tabular}

\subsection{Cells}

\begin{tabular}{|l|l|l|l|}
\hline Name & Species & Organ & Source \\
\hline FOB 1.19 & human & bone & T. Spelsberg, Mayo Clinic, USA (Harris et al., 1995) \\
\hline HEK293T & human & kidney & V. Assmann, UKE, Hamburg \\
\hline MSC & human & bone & M. Kassem, SDU, Denmark (Simonsen et al., 2002) \\
\hline
\end{tabular}




\subsection{ChIP-seq datasets}

\begin{tabular}{|l|l|l|l|}
\hline Cell line & ChIP & Antibody & Source \\
\hline FOB & H3K27ac & $\begin{array}{l}\text { Diagenode, pAB- } \\
196\end{array}$ & Z. Najafova (AG Johnsen) \\
\hline MSC & H3K4me3 & $\begin{array}{l}\text { Diagenode Mab- } \\
003\end{array}$ & M. Hennion (AG Johnsen) \\
\hline MSC & H3K27ac & $\begin{array}{l}\text { Diagenode, pAB- } \\
196\end{array}$ & Z. Najafova (AG Johnsen) \\
\hline
\end{tabular}

\subsection{Software}

\begin{tabular}{|l|l|}
\hline Name & Developer \\
\hline Bio-Rad CFX Manager 3.1 & Bio-Rad Laboratories, Hercules, USA \\
\hline Bowtie1 Version 1.1.1 & Langmead B. et al., 2009 \\
\hline Bowtie2 Version 2.1.0 & Langmead B. et al., 2012 \\
\hline CEAS Version 1.0.2 & Shin et al., 2009 \\
\hline Cistrome & Liu et al., 2011 \\
\hline Deeptool Galaxy Server 1.5.9.1.0 & Ramirez et al., 2014 \\
\hline FastQC Version 0.11.2 & S. Andrews (Babraham Institute) \\
\hline Image Lab Version 5.2 build 14 & Bio-Rad Laboratories, Hercules, USA \\
\hline Integrative Genome Viewer 2 & James T. Robinson et al., 2011 \\
\hline MACS2 Version 2.1.0. & Zhang et al., 2008 \\
\hline Primer designing tool NCBI/Primer-BLAST & Ye et al., 2012 \\
\hline SAMtools Version 0.1.19 & Li et al., 2009 \\
\hline Statistical software R, Version 3.1.1 & R Development Core Team 2008 \\
\hline useGalaxy Version 15.07 & Giardine et al., 2005 \\
\hline
\end{tabular}

\begin{tabular}{|l|l|}
\hline R-packages & Developer \\
\hline DESeq1 V1.16.0 - Bioconductor package & Anders and Huber, 2010 \\
\hline DiffBind V1.10.2 - Bioconductor package & $\begin{array}{l}\text { Start and Brown, 2012, Ross-Innes et } \\
\text { al., 2012 }\end{array}$ \\
\hline
\end{tabular}




\subsection{Buffers and media}

Blocking solution: 1 x TBS-T, $5 \%(\mathrm{w} / \mathrm{v})$ milk

Cell culture freezing medium: 42\% (v/v) DMEM, 50\% (v/v) FBS, 8\% DMSO

ChIP crosslinking buffer: $1 \%$ or $1.42 \%$ Formaldehyde in PBS

ChIP IP buffer: $150 \mathrm{mM} \mathrm{NaCl}, 5 \mathrm{mM}$ EDTA, $50 \mathrm{mM}$ Tris (pH 8), 0.5\% (v/v) NP-40, $1 \%(v / v)$ Triton X-100

ChIP Wash buffer: 500 mM LiCl, 20 mM EDTA, 100 mM Tris (pH 8.5), 1\% (v/v) NP40, $20 \mathrm{mM} \mathrm{NaF}, 1 \%(\mathrm{w} / \mathrm{v})$ Sodium deoxycholate

Dexamethasone stock solution: $100 \mu \mathrm{M}$ Dexamethasone in 100\% EtOH

Dilution buffer: $0.01 \%(\mathrm{w} / \mathrm{v})$ SDS, 1.1\% (v/v) Triton X-100, $1.2 \mathrm{mM}$ EDTA, $16.7 \mathrm{mM}$ Tris- $\mathrm{HCl}(\mathrm{pH} 8.1), 167 \mathrm{mM} \mathrm{NaCl}$

DMEM cell culture medium: phenol red-free supplemented with 10\% FBS, $100 \mathrm{U} / \mathrm{ml}$ penicillin, $100 \mu \mathrm{g} / \mathrm{ml}$ streptomycin

DMEM-F12 cell culture medium: phenol red-free, high-glucose, supplemented with 10\% FBS, $100 \mathrm{U} / \mathrm{ml}$ penicillin, $100 \mu \mathrm{g} / \mathrm{ml}$ streptomycin

Lämmli buffer (6x): $0.35 \mathrm{M}$ Tris (pH 6.8), 30\% (v/v) glycerol, 10\% (w/v) SDS, 9.3\% (w/v) DTT, 0.02\% (w/v) bromphenol blue

MEM a cell culture medium: phenol red, L-glutamine, supplemented with $10 \%$ FBS, $100 \mathrm{U} / \mathrm{ml}$ penicillin, $100 \mu \mathrm{g} / \mathrm{ml}$ streptomycin

Nuclear preparation buffer: $150 \mathrm{mM} \mathrm{NaCl}, 20 \mathrm{mM}$ EDTA, $50 \mathrm{mM}$ Tris (pH 7.5), $0.5 \%$ (v/v) NP-40, 1\% (v/v) Triton X-100, $20 \mathrm{mM} \mathrm{NaF}$

PBS for cell culture: 1 PBS tablet per $500 \mathrm{ml} \mathrm{ddH}_{2} \mathrm{O}$

PBS: $137 \mathrm{mM} \mathrm{NaCl}, 2.68 \mathrm{mM} \mathrm{KCl}, 4.29 \mathrm{mM} \mathrm{Na}_{2} \mathrm{HPO}_{4} \times 2 \mathrm{H}_{2} \mathrm{O}, 1.47 \mathrm{mM} \mathrm{KH}_{2} \mathrm{PO}_{4}$, $(\mathrm{pH} 7.4)$

PBS-T: PBS including 0.1\% (w/v) Tween-20

PCI: Phenol: Chloroform: Isoamylalcohol (25:24:1)

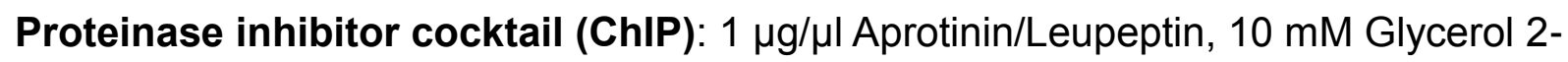
phosphate disodium salt hydrate, $1 \mathrm{mM} \mathrm{N}$-Ethylmaleimide, $1 \mathrm{mM}$ Pefabloc, $1 \mathrm{mM}$ $\mathrm{NiCl}_{2}$ and $10 \mu \mathrm{M}$ indole acetamide

Proteinase inhibitor cocktail (WB): $1 \mathrm{ng} / \mu \mathrm{l}$ Aprotinin/Leupeptin, $10 \mathrm{mM}$ Glycerol 2phosphate disodium salt hydrate, $1 \mathrm{mM}$ NEM, $1 \mathrm{mM}$ Pefabloc

qPCR buffer: $75 \mathrm{mM}$ Tris- $\mathrm{HCl}(\mathrm{pH} 8.8), 20 \mathrm{mM}\left(\mathrm{NH}_{4}\right)_{2} \mathrm{SO}_{4}, 0.01 \%$ Tween-20, $3 \mathrm{mM}$ $\mathrm{MgCl}_{2}, 200 \mu \mathrm{M}$ dNTPs, $0.5 \mathrm{U} /$ reaction Taq DNA Polymerase, 0.25\% Triton X-100, 1: 80,000 SYBR Green I, 300 mM Trehalose and 30 nM primer 
RIPA buffer: $1 \times$ PBS, $1 \%(\mathrm{v} / \mathrm{v})$ NP-40, 0.5\% (v/v) sodium deoxychelate, $0.1 \%(\mathrm{w} / \mathrm{v})$ SDS

SDS separating gel (15\%): 15\% (v/v) acrylamide, $375 \mathrm{mM}$ Tris- $\mathrm{HCl}(\mathrm{pH} 8.8), 0.1 \%$ (w/v) SDS, $0.1 \%(w / v)$ APS, $0.04 \%$ (v/v) TEMED

SDS separating gel (6\%): 6\% (v/v) acrylamide, $375 \mathrm{mM}$ Tris- $\mathrm{HCl}(\mathrm{pH} 8.8), 0.1 \%$ (w/v) SDS, $0.1 \%(w / v)$ APS, $0.04 \%$ (v/v) TEMED

SDS stacking gel (5\%): $5 \%(\mathrm{v} / \mathrm{v})$ acrylamide, $125.5 \mathrm{mM}$ Tris- $\mathrm{HCl}(\mathrm{pH} 6.8), 0.1 \%$ (w/v) SDS, $0.1 \%(w / v)$ APS, $0.1 \%(v / v)$ TEMED

Sodium acetate: $3 \mathrm{M}$ sodium acetate, $(\mathrm{pH} 5.2)$

Sonication buffer 1: $10 \mathrm{mM}$ EDTA, $50 \mathrm{mM}$ Tris (pH 8), 1\% (w/v) SDS

Sonication buffer 2: $300 \mathrm{mM} \mathrm{NaCl}, 40 \mathrm{mM}$ EDTA, $100 \mathrm{mM}$ Tris (pH 7.5), 2\% (v/v) NP-40, 40 mM NaF

TAE buffer (50x): $2 \mathrm{M}$ Tris, $1 \mathrm{M}$ Acetic acid, $0.1 \mathrm{M}$ EDTA

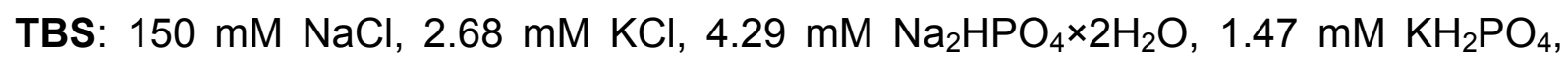
$(\mathrm{pH} 7.4)$

TBS-T: TBS including $0.1 \%(\mathrm{w} / \mathrm{v})$ Tween-20

TE buffer: $10 \mathrm{mM}$ Tris-HCl, 1 mM EDTA, (pH 8.0)

Tris-glycine electrophoresis buffer: $25 \mathrm{mM}$ Tris, $200 \mathrm{mM}$ Glycine, 0.1\% (w/v) SDS

Western blot transfer buffer: 10\% (v/v) 10× Western salts, 20\% (v/v) Methanol

Western salts (10x): $1.92 \mathrm{M}$ Glycine, $250 \mathrm{mM}$ Tris- $\mathrm{HCl}(\mathrm{pH} 8.3), 0.02 \%(w / v)$ SDS 


\section{Methods}

\subsection{Cell culture}

\subsubsection{Cell culturing}

Bone marrow derived human mesenchymal stem cells (MSC) and human fetal osteoblast cells (FOB) were cultured at $37^{\circ} \mathrm{C}$ and $34^{\circ} \mathrm{C}$, respectively, under $5 \% \mathrm{CO}_{2}$ atmosphere and maintained sub-confluent. MSC were grown in alpha modified Eagle's medium (aMEM) supplemented with 10\% fetal bovine serum (FBS), 100 units $/ \mathrm{ml}$ penicillin, $100 \mu \mathrm{g} / \mathrm{ml}$ streptomycin and the antifungal agent Fungizone® Antimyotic $(25 \mu \mathrm{g} / \mathrm{ml})$ (growth medium). Stable pGIPZ transduced MSC were grown in growth medium supplemented with $1 \mu \mathrm{g} / \mathrm{ml}$ puromycine (selection medium). FOB were cultured in Dulbecco's modified Eagle's medium-F12 (DMEM-F12) supplemented like MSC.

\subsubsection{Adipocyte and osteoblast differentiation}

Osteoblast differentiation of confluent cells was induced by addition of $10 \mathrm{mM} \beta$ glycerophosphate, $0.2 \mathrm{mM}$ ascorbate, $10 \mathrm{nM}$ calcitriol and $100 \mathrm{nM}$ dexamethasone into the growth medium (osteoblast differentiation medium). FOB were shifted $4 \mathrm{~h}$ prior to the addition of the osteoblast differentiation mix from $34{ }^{\circ} \mathrm{C}$ to $39{ }^{\circ} \mathrm{C}$ to inactivate the stably integrated Large-T antigen, which drives proliferation. For adipocyte differentiation confluent cells were cultured in normal growth medium supplemented with $15 \%$ FBS and $2 \mu \mathrm{M}$ insulin, $0.45 \mathrm{mM}$ isobutylmethyl-xanthine, 10 $\mu \mathrm{M}$ troglitazone and $100 \mathrm{nM}$ dexamethasone (adipocyte differentiation medium). The differentiation medium was changed every second day.

\subsubsection{Reverse transfection}

Small interfering RNA (siRNA) was transfected with Lipofectamine ${ }^{\circ}$ RNAiMAX according to the manufacturers reverse transfection protocol. Briefly, $30 \mathrm{pmol}$ of siRNA were mixed together with $5 \mu$ l RNAiMAX reagent in $500 \mu \mathrm{l}$ of optiMEM and incubated for $20 \mathrm{~min}$ at room temperature. In the meantime, cells were trypsinized and suspended in growth medium without antibiotics or antifungal reagents supplemented (transfection medium). Then 250,000 cells, counted in a Neubauer counting chamber, were seeded in $1.5 \mathrm{ml}$ of transfection medium into a well of a 6 well plate. After $20 \mathrm{~min}$ of incubation time the transfection mix was added directly to the medium and incubated for $16 \mathrm{~h}$ on the cells. Thereafter the medium was changed 
to normal growth medium. For transfections in 10 or $15 \mathrm{~cm}$ plates each factor in the mix was scaled up by a factor of 4 or 8 respectively.

\subsubsection{Forward transfection}

During a differentiation experiment for more than 4 days the cells were transfected again 3 days after the last transfection with siRNA by Lipofectamine ${ }^{\circledR}$ RNAiMAX based on the manufacturer forward transfection protocol. Per Well of a 6-well plate 35 pmol of siRNA were mixed with $7.5 \mu$ RNAiMAX in $500 \mu$ optiMEM and incubated for $20 \mathrm{~min}$ at room temperature. Meanwhile the growth medium on the cells was removed and replaced with $1 \mathrm{ml}$ of transfection medium. 20 min after incubation the transfection mix was added directly into the medium and replaced by the respective differentiation medium after 6 to $8 \mathrm{~h}$.

\subsubsection{Generation of stable cell lines by lentiviral infection}

A microRNA-adapted short hairpin RNA (shRNA), coded on the GIPZ plasmid was stable integrated into the genome by lentiviral transformation. Prior to production of the lentiviral particles the shRNA expressing sequence on the GIPZ plasmid was checked by Sanger sequencing. For the viral particle production human embryonic kidney 293 cells (HEK293T) cells were used. In the following the production of stable cell lines is described.

\subsubsection{Transfection of HEK293T cells}

$80 \%$ confluent HEK293T cells grown in a $10 \mathrm{~cm}$ plate were transfected with the GIPZ, lentiviral packaging and envelope plasmid by Polyethylenimine (PEI) transfection with the following mix:

\begin{tabular}{|c|c|}
\hline Reagent & Amount \\
\hline Vector plasmid pGIPZ & $15 \mu \mathrm{g}$ \\
\hline Packaging plasmid psPAX2 & $10 \mu \mathrm{g}$ \\
\hline Envelope plasmid pMD2.G & $5 \mu \mathrm{g}$ \\
\hline Transfection reagent PEI & $50 \mu \mathrm{g}$ \\
\hline Transfection solution optiMEM & $1.1 \mathrm{ml}$ \\
\hline
\end{tabular}


First, the plasmids were added into the optiMEM solution and then mixed with PEI. The mixture was vortexed for approximately 8 secs and incubated then for $10 \mathrm{~min}$ at room temperature. Within the incubation time the medium on the cells was replaced with $6 \mathrm{ml}$ of transfection medium. $1 \mathrm{ml}$ of the transfection mix was pipetted dropwise to the medium of the HEK293T cells. The cells were subsequently incubated for $14-$ $16 \mathrm{~h}$ before the medium was replaced with normal growth medium. Two days after transfection the constitutive expression of GFP, encoded by the transfected GIPZ plasmid, was controlled by fluorescence microscopy.

\subsubsection{Collection of viral supernatant}

With a transfection efficiency greater than $70 \%$ the viral particles were harvested by centrifugation of the supernatant at $300 \mathrm{~g}$ for $3 \mathrm{~min}$ and subsequent filtered through a $0.45 \mu \mathrm{m}$, non-pyrogenic filter. The filtered supernatant was aliquot and stored for either up to $24 \mathrm{~h}$ at $4{ }^{\circ} \mathrm{C}$ or was snap frozen in liquid nitrogen and stored at $-80^{\circ} \mathrm{C}$.

\subsubsection{Lentiviral infection of cells}

For the lentiviral infection of cells $50-70 \%$ confluent MSC or FOB grown in a $10 \mathrm{~cm}$ plate were infected by addition of $1 \mathrm{ml}$ virus particle supernatant into antibiotic free growth medium supplemented with $8 \mu \mathrm{g} / \mathrm{ml}$ polybrene. After $24 \mathrm{~h}$ were the cells washed two times with PBS and normal growth medium was added. The infection and stable integration of the PGIPZ part were controlled by puromycine selection, which resistance gene is encoded on the integrative part. Cells were thereafter maintained in selection medium.

\subsection{Chemical staining}

\subsubsection{Oil Red O staining}

Oil Red O staining was used to stain lipid droplets of adipocyte differentiated cells. Therefore, cells were washed once with PBS and fixed in a $10 \%$ formalin solution in PBS for $30 \mathrm{~min}$ at room temperature. Meanwhile the staining solution was prepared by mixing 3 parts of Oil Red $O$ stock solution with 2 parts of deionized water. The mixture was incubated for $10 \mathrm{~min}$ at room temperature and filtered through a $0.45 \mu \mathrm{m}$ pore filter. After fixation the cells were washed twice with deionized water and incubated while covered with $60 \%$ isopropanol for $5 \mathrm{~min}$. Thereafter the isopropanol was replaced with the staining solution and incubated for $5 \mathrm{~min}$. Finally, the cells were washed carefully with deionized water until the water rinsed off clear. Pictures 
of the stained cells were taken with a microscope operated at 10 - or 20 -fold magnification.

\subsubsection{Alkaline phosphatase staining}

Osteoblast differentiated cells were stained for alkaline phosphatase activity. The staining solution was prepared by dissolving $5 \mathrm{mg}$ of Naphtol AS-MX in $200 \mu \mathrm{l}$ of NNDimethyl formamide. The solution was gently mixed and pipetted into $50 \mathrm{ml}$ of 100 $\mathrm{mM}$ Tris- $\mathrm{HCl} \mathrm{pH} 8.4$ solution. $30 \mathrm{mg}$ of Fast Blue RR salt were added subsequently to the mixture and vortexed until the solution was homogeneous. Finally, the staining solution was filtrated through a $0.45 \mu \mathrm{m}$ pore filter. The cells were fixed for $15 \mathrm{~min}$ with $4.8 \%$ formaldehyde in PBS. Subsequently the cells were washed two times with PBS and covered with the staining solution for a minimum of $15 \mathrm{~min}$ in the dark. Afterwards the cells were washed carefully with deionized water until the water rinsed off clear. The stained cells were either scanned within the whole well or images were taken at 10 - or 20 -fold magnification on a microscope.

\subsection{Ectopic bone formation experiment}

The ectopic bone formation experiment was performed in the group of Prof. Dr. Moustapha Kassem, University of Southern Denmark by Nicholas Ditzel. For the experiment three different stable transformed MSC cell lines with either two individual shRNA directed against CHD1 or one non-targeting control shRNA were sent on dry ice. The experiment was performed as described (Chen, L. and Ditzel, N., 2015).

\subsection{Molecular biology}

\subsubsection{RNA isolation}

RNA isolation was performed according to the manufacturers' instructions. For cells grown in a 6-well plates $500 \mu \mathrm{l}$ of QIAzol reagent was added to the cells and incubated for $3 \mathrm{~min}$ at room temperature. Afterwards the cells were scraped and transferred to a reaction tube with $100 \mu \mathrm{l}$ Chloroform and vortexed for $15 \mathrm{sec}$. The mix was centrifuged for $15 \mathrm{~min}$ at $12,000 \mathrm{~g}$ and subsequently the aqueous supernatant was transferred to a fresh tube with $99 \%$ Isopropanol in a 1:1 ratio. The solution was incubated overnight at $-20{ }^{\circ} \mathrm{C}$. The next day the solution was centrifuged for $30 \mathrm{~min}$ at $15,000 \mathrm{~g}$ and subsequently washed two times with $70 \%$ ethanol at $12,000 \mathrm{~g}$. The RNA pellet was air dried and solved in $30 \mu \mathrm{l}$ nuclease free water. The 
concentration was measured on a Nanodrop and used for complementary DNA synthesis or for RNA-sequencing library preparation.

\subsection{2 complementary DNA synthesis}

$1 \mu \mathrm{g}$ of RNA was diluted in $10 \mu \mathrm{l}$ of nuclease free water and supplemented with $2 \mu \mathrm{l}$ of $15 \mu \mathrm{M} 9$ mer random primer and $4 \mu \mathrm{l}$ of $2.5 \mathrm{mM}$ dNTP to a volume of $16 \mu \mathrm{l}$. The mix was incubated for $5 \mathrm{~min}$ at $70{ }^{\circ} \mathrm{C}$ and then placed immediately on ice. After the samples cooled down $0.125 \mu \mathrm{l}$ of $25 \mathrm{U}$ MMLV-reverse transcription enzyme and 0.25 $\mu$ of $10 \cup$ Murine RNase-Inhibitor were added together with the reverse-transcription buffer to a final volume of $20 \mu \mathrm{l}$. The mix was incubated for $1 \mathrm{~h}$ at $42{ }^{\circ} \mathrm{C}$ and subsequently heat inactivated at $90{ }^{\circ} \mathrm{C}$ for $10 \mathrm{~min}$. The complementary DNA (cDNA) was diluted to $50 \mu \mathrm{l}$ with nuclease free water and used for real-time quantitative PCR (qPCR).

\subsection{3 qPCR reaction}

Per reaction either $1 \mu \mathrm{l}$ of cDNA or diluted DNA of a ChIP experiment was mixed with $24 \mu \mathrm{l}$ of qPCR buffer. Samples were pipetted in technical duplicates per qPCR measurement. For amplification and read out the following qPCR program was used:

\begin{tabular}{|c|c|c|c|}
\hline Step & Temperature & Time & Number of cycles \\
\hline Step 1 & $95^{\circ} \mathrm{C}$ & $2 \mathrm{~min}$ & 1 \\
\hline Step 2 & $95^{\circ} \mathrm{C}$ & $15 \mathrm{sec}$ & $40-45$ \\
\hline Step 3 & $60^{\circ} \mathrm{C}$ & $1 \mathrm{~min}$ & \\
\hline
\end{tabular}

For PCR amplification step two and three were repeated 40 or 45 times for cDNA or ChIP samples respectively. Subsequent to the PCR amplification a melting curve of the PCR products was measured by a read out every $0.5^{\circ} \mathrm{C}$ increasing from $60^{\circ} \mathrm{C}$ to $90{ }^{\circ} \mathrm{C}$. Signal intensities of the samples were read out by quantification relative to a dilution curve. Gene expression values were divided over unaffected control genes (RPLPO and 18S rRNA) and averaged for at least biological triplicates. 


\subsubsection{Chromatin immunoprecipitation}

Chromatin immunoprecipitation (ChIP) was performed according to a modified protocol of (Gomes et al., 2006; Nelson et al., 2006). All ChIP experiments were performed in biological triplicates.

\subsubsection{Crosslinking}

First protein-protein and DNA-protein interactions were cross-linked with 1 or $1.42 \%$ formaldehyde in PBS for $10 \mathrm{~min}$ at room temperature. $1.42 \%$ formaldehyde concentration was used to ChIP CHD1, all other ChIP crosslinking reactions were performed with $1 \%$ formaldehyde concentration. The crosslinking reaction was quenched for $5 \mathrm{~min}$ by addition of glycine to a final concentration of $125 \mathrm{mM}$. The cells were washed two times with ice-cold PBS and scraped in nuclear preparation buffer supplemented with the ChIP inhibitors cocktail. The cell nuclei were extracted by careful mixing and subsequent centrifugation at $10,000 \mathrm{~g}$ for $5 \mathrm{~min}$. This step was repeated one time. Thereafter the cell nuclei pellet was either snap frozen in liquid nitrogen and stored at $-80^{\circ} \mathrm{C}$ or further processed.

\subsubsection{Sonication}

The chromatin was sheared to 300 to $600 \mathrm{bp}$ fragments by ultrasound sonication. Therefore, the nuclei were resuspended in $120 \mu \mathrm{l}$ sonication buffer 1 (+ proteinase inhibitor cocktail) and incubated for $10 \mathrm{~min}$ on a rotating wheel at $4{ }^{\circ} \mathrm{C}$. Notably, for CHD1 ChIP the concentration of the sonication buffer 1 was decreased to $0.2 \%$. After incubation $120 \mu$ sonication buffer 2 (+ proteinase inhibitor cocktail) was added and the suspension was mixed well. Subsequently the chromatin was sonicated with the Bioruptor ${ }^{\circledR}$ for 30 cycles, each cycle containing a $30 \mathrm{sec}$ sonication pulse and a 30 sec pause. For the CHD1-ChIP the chromatin was sheared for 60 cycles.

\subsubsection{Size control of sonicated fragments}

The shearing process was controlled by isolation of the DNA and agarose gel electrophoresis. $5 \%$ of the sheared chromatin extract was added to $100 \mu \mathrm{l}$ of sonication buffer 1 plus $1 \mu \mathrm{l}$ proteinase $\mathrm{K}(20 \mu \mathrm{g} / \mu \mathrm{l})$ and incubated over night at 65 ${ }^{\circ} \mathrm{C}$, shaking at $800 \mathrm{rpm}$. The next day $100 \mu \mathrm{l}$ deionized water, $10 \mu \mathrm{LiCl}(8 \mathrm{M}), 2 \mu \mathrm{l}$ linear polyacrylamide $(5 \mu \mathrm{g} / \mu \mathrm{l})$ were mixed to isolate the DNA by a Phenol/Chloroform-/ Isoamyl (PCl) extraction. $\mathrm{PCl}$ was added in a one to one ratio to the solution, vortexed for 30 secs and centrifuged at $16,000 \mathrm{~g}$ for $2 \mathrm{~min}$. The aqueous supernatant was transferred into a fresh tube and another extraction from the 
remaining $\mathrm{PCl}$ solution was performed by adding $200 \mu \mathrm{l} 10 \mathrm{mM}$ Tris- $\mathrm{HCl} \mathrm{pH} 8$ and repetition of the first step of the $\mathrm{PCl}$ extraction. The supernatant was added to the first one, mixed with $99 \% \mathrm{EtOH}$ in a $1: 2.5$ ratio and incubated over night at $-20{ }^{\circ} \mathrm{C}$. The DNA was precipitated by centrifugation at $15,000 \mathrm{~g}$ at $4{ }^{\circ} \mathrm{C}$ for 30 min with one wash step afterwards by addition of $70 \% \mathrm{EtOH}$ and subsequent centrifugation. The pellet was air dried and dissolved then in $10 \mathrm{mM}$ Tris $\mathrm{pH}$ 8, containing RNaseA (0.1 $\mu \mathrm{g} / \mu \mathrm{l})$ and incubated for $1 \mathrm{~h}$ at $37^{\circ} \mathrm{C}$. The DNA fragment size was analyzed by a HD-green $1.5 \%$ agarose gel.

\subsubsection{Chromatin immunoprecipitation}

After control of the correct DNA fragment size the specific protein-DNA complexes were immunoprecipitated. First, unspecific binding to sepharose was decreased by addition of $100 \mu \mathrm{l} 50 \%$ slurry sepharose in a total volume of $1 \mathrm{ml}$ of dilution buffer (+ proteinase inhibitor cocktail). The extracts were incubated for $1 \mathrm{~h}$ at $4{ }^{\circ} \mathrm{C}$ on a rotating wheel. After removal of the sepharose by centrifugation at 10,000 for $5 \mathrm{~min}$, the cleared chromatin extracts were split for ChIP, unspecific IgG control and a 10\% input sample (for dilutions of antibodies for ChIP see Material - 2.6.3.1). The ChIP and control samples were filled up with immuno-precipitation buffer (IP-buffer) (+ proteinase inhibitor cocktail) to a total volume of $800 \mu$ and incubated overnight on a rotating wheel at $4{ }^{\circ} \mathrm{C}$. After $14-18 \mathrm{~h}$ incubation $30 \mu \mathrm{l}$ of $50 \%$ slurry protein-A or protein-G sepharose coupled beads, for rabbit or mouse antibody respectively, were added, and incubated for $2 \mathrm{~h}$ on a rotating wheel at $4{ }^{\circ} \mathrm{C}$. After incubation the beads were washed three times with IP-buffer, two times with ChIP wash buffer, three times with IP-buffer again and finally two times with TE-buffer. Between each step the coupled beads were mixed carefully and centrifuged for $2 \mathrm{~min}$ at $2,000 \mathrm{~g}$ at $4{ }^{\circ} \mathrm{C}$.

\subsubsection{Isolation of precipitated DNA}

The precipitated chromatin was treated with RNAseA $(0.2 \mu \mathrm{g} / \mu \mathrm{l})$ at $37^{\circ} \mathrm{C}$ for $30 \mathrm{~min}$ and subsequently the proteins were digested by addition of $1 \mu \mathrm{l}$ Proteinase- $\mathrm{K}$ ( 20 $\mu \mathrm{g} / \mathrm{ml}$ ) incubating overnight on a shaker at $65^{\circ} \mathrm{C}$ at $800 \mathrm{rpm}$. The next day the DNA was isolated by $\mathrm{PCl}$ extraction identical as performed previously (see 3.3.4.3) and transferred afterwards to DNA low-binding tubes to precipitate overnight. The next day the solution was centrifuged at $15,000 \mathrm{~g}$ for $30 \mathrm{~min}$ at $4{ }^{\circ} \mathrm{C}$, washed two times with $70 \% \mathrm{EtOH}$ and the pellet was subsequently air dried. The pellet was solved in $20 \mu \mathrm{l}$ of nuclease free water and the DNA concentration was measured by Qubit $\AA$ 
assay according to manufacturer's protocol. For ChIP-qPCR the DNA was diluted in a ratio of 1:5.

\subsection{Protein analysis}

\subsubsection{Sample preparation}

For protein extraction the cells were scraped in ice cold RIPA-Buffer supplemented with the proteinase inhibitor cocktail. The cell lysate was sonicated either by Bandelin Sonoplus tip sonicator for 15 pulses, $10 \%$ intensity $3 \mathrm{sec}$ on and off, or by the Diagenode Bioruptor for 15 min high intensity with 30 secs on and off. Before size separation of the proteins the sheared whole cell lysates were diluted with Laemmli buffer to $1 \mathrm{x}$ and heated for $10 \mathrm{~min}$ at $95{ }^{\circ} \mathrm{C}$. The samples were loaded to an appropriately concentrated polyacrylamide gel respective for the protein size to separate (PAA-gel). The samples and a control protein size ladder were separated by applying constant $100 \mathrm{~V}$.

\subsubsection{Western blot and immunostaining}

Subsequent to gel electrophoresis the PAA-gel was transferred to a polyvinylidene difluoride (PVDF) membrane using the wet electro blot system. The electro blotting was performed in Western blot transfer buffer according to the manufacturer's instructions. After set up, the proteins were blotted to the membrane for $1.5 \mathrm{~h}$ with $100 \mathrm{~V}$. After the transfer, the membrane was stained with Ponceau-S and blocked for unspecific antibody binding in $5 \%$ skim-milk in TBS-T for $1 \mathrm{~h}$ at room temperature. Next, the membrane was incubated with the primary antibody in a $5 \%$ skim-milk TBS-T solution over night at $4{ }^{\circ} \mathrm{C}$ on a rotator within a $50 \mathrm{ml}$ reaction tube. The next day the membrane was washed 3 times for $10 \mathrm{~min}$ in TBS-T and incubated subsequently for 1 hour on a shaker with a secondary horseradish peroxidase (HRP) coupled antibody directed against the primary antibody. After incubation the membrane was washed 3 times in TBS-T and the chemiluminescence signal of the HRP was monitored by an X-ray film or a gel imaging system. 


\subsection{Next generation sequencing}

\subsubsection{Library preparation}

RNA and DNA were processed by library preparation for unstranded sequencing in biological duplicates for RNA-seq and triplicates for ChIP-seq. In the following the different library preparation protocols for RNA and DNA are described.

\subsubsection{RNA-library preparation}

Previous to the RNA library preparation the sample quality was controlled for its RNA integrity. Therefore, the RNA was separated by size with a RNA Nano chip on a Bioanalyzer. To estimate the integrity of the RNA a program calculated a RNA integrity number (RIN) based on degradation products and the ratio between the $28 S$ rRNA and 18S rRNA. If the samples had a RIN greater or equal to 7 they were processed for the RNA library preparation. The preparation was performed as described in the NEBNext ${ }^{\circledR}$ Ultra ${ }^{\mathrm{TM}}$ Library Prep Kit. Briefly, $1 \mu \mathrm{g}$ of total RNA was measured by Nanodrop and used as starting material. The mRNA was isolated by pull down via polyadenylated coated magnetic beads. After several washing steps the mRNA was fragmented at $94{ }^{\circ} \mathrm{C}$ and applied for the first and subsequently second strand cDNA synthesis. Single base overhangs at the $5^{\prime}$-ends were repaired and sequencing adaptors were ligated to the cDNA strands. To barcode the samples, the processed cDNA was amplified by PCR with one common and one individual primer. This allows later during sequencing to distinguish individual samples in one sequencing lane. Subsequently the DNA concentration was measured by Qubit ${ }^{\circledR}$ Fluorometer and the length of the library fragments were detected on a Bioanalyzer by a High Sensitivity DNA chip.

\subsubsection{DNA-library preparation}

The library preparation for ChIP DNA was performed with the MicroPlex ${ }^{\mathrm{TM}}$ Library Preparation Kit v2 from Diagenode ${ }^{\circledR}$ and used according to the manufacturer's protocol. The CHD1 ChIP DNA library preparation was performed using the NEBNext ${ }^{\circledR}$ Ultra ${ }^{\mathrm{TM}}$ DNA Library Prep Kit for Illumina ${ }^{\circledR}$ according to the manufacturer's protocol. As starting material an equal amount of DNA (between 2 $10 \mathrm{ng}$ ) per ChIP condition was used. Prior to library preparation the DNA was sheared on the Bioruptor for 30 cycles, each cycle divided into 30 sec sonication and $30 \mathrm{sec}$ pause. The library preparation was performed similar to the RNA library preparation protocol above, except the polyadenylated bead purification, heat 
fragmentation and reverse transcription of RNA to cDNA, which is described in more detail in the manufacturer's protocol.

\subsubsection{Sequencing}

For sequencing up to 12 samples were pooled to a final concentration of $10 \mathrm{~nm}$ or 2 $\mathrm{nm}$ depending on the lowest concentrated sample after the library concentration. The samples were sequenced on Illumina ${ }^{\circledR}$ HiSeq 2000 sequencers at the HeinrichPette-Institute, Hamburg or the Transcriptome Analysis Laboratory (TAL), Göttingen.

\subsection{Bioinformatic processing of sequencing data}

\subsubsection{Mapping of ChIP-seq reads to the genome}

The ChIP sequencing reads received from the sequencing facilities or reads downloaded from public available datasets (see Material 2.8 ChIP-seq datasets) were controled for their quality by FASTQ quality check (FastQC) (S. Andrews Babraham Institute) and mapped to the human genome (hg 19, GRCh37) via Bowtie1 (Langmead et al., 2009). Bowtie1 parameters were set to parameters $-\mathrm{m} 1-\mathrm{k} 1$. The output files in sequence alignment format /map (SAM) were then transformed to binary alignment format/ map (BAM) by the software package SAMtools ( $\mathrm{Li}$ et al., 2009).

\subsubsection{Peak calling via MACS2}

Mapped and BAM formatted reads were analyzed for significantly, above background enriched genome regions by the peak calling software model-based analysis of ChIPseq 2 (MACS2) (Zhang et al., 2008). The background was defined by the sequenced input. As parameter the genome (-g) was set to hs (homo sapiens), the q-value (-q) was set to 0.05 and the input format $(-f)$ was specified to BAM. The narrowpeak option was used for all datasets except RNA-Pol II and H2Bub1. For the peak calling the BAM files of the samples were merged for each condition.

\subsubsection{RNA-seq analysis}

The sequenced RNA in FASTQ format were mapped to the human reference transcriptome (UCSC hg19) by Bowtie2 with default parameters (Langmead and Salzberg, 2012). The reads per gene were counted by a homemade script, kindly provided by Malik Alawi, UKE Hamburg, Bioinformatic service facility. Subsequently the raw reads were normalized by the DESeq package using the default protocol parameters (Anders and Huber, 2010). For further analysis of the DESeq output 
matrix R statistical software or Microsoft Office Excel was used (R Development Core Team, 2008).

\subsubsection{Normalization and calculation of ChIP-seq binding affinities}

BAM formatted files were uploaded to the public available deepTools server to normalize the sequencing reads either by the bamCompare or bamCoverage tools (Ramírez et al., 2014).. The bamCompare tool calculates a ratio between input and ChIP-seq file over all genomic regions subset to defined region sizes (bins). The parameter was set as default with a bin size of $50 \mathrm{bp}$. Specific genomic loci, described by the ENCODE Project Consortium as artificial, high signal regions, were excluded for calculations for the scaling factor. Reads were normalized by reads per genomic content (RPGC) to a $1 x$ depth of coverage. Therefore, the total number of mapped reads were multiplied with the fragment length and divided by the effective genome size (2.7 billion). Further settings were set as default in the bamCoverage tool. To calculate the ChIP-seq signal strength at defined genomic regions the tool computeMatrix was used with default settings. The visualization of the computed matrix was done either by the profiler (aggregation plot) or heatmapper (heatmaps).

\subsubsection{Visualization by IGV}

To visualize profiles of normalized sequencing intensities on the genome at individual regions the Integrative Genome Viewer software was used (Robinson et al., 2011). As input bamCoverage files were used. Tracks were if necessarily overlaid.

\subsubsection{Analysis in $\mathbf{R}$}

For subset and filtering of datasets as well as calculation of Boxplots and scatter plots the R statistical software was used. For statistical test of non-parametric distributions, the Wilcoxon Rank-Sum test was performed with default conditions set in R. For parametric distributions the Welch's t-test was used. Correlation coefficiencies were calculated by the Pearson method with default parameter set in $\mathrm{R}$.

\subsubsection{Cis-regulatory element annotation system}

By cis-regulatory element annotation system (CEAS) software quantitatively representation of protein binding sites within genomic functional regions were represented (Shin et al., 2009). As an input BED and bigwig files received after MACS2 analysis were used in CEAS with default parameters for the human genome (hg19). 


\subsubsection{DAVID based analysis of RNA-sequencing}

The database for annotation, visualization and integrated discovery (DAVID) software was used for functional annotation of gene ontology (GO) terms (Huang et al., 2009). As a background file the default human genome file within the DAVID software was used.

\subsubsection{DiffBind and calculation of RNA-Pol II stalling ratios}

DiffBind is a program, which was used to calculate ChIP-seq biding intensities at defined genomic regions with statistical differential binding analysis for biological replicates (Ross-Innes et al., 2012). For calculating the stalling ratio of RNA-Pol II in siCon or siCHD1 treated FOBs the occupancy of RNA-Pol II was measured at TSSregions as well as the gene body similar as previously described in Lin et al., 2012. Briefly, regions $300 \mathrm{bp}$ up- and downstream of the TSS as well as the gene body were used as input files in bed format for the DiffBind software. The gene body was defined as the region between $30 \%$ of the gene length downstream of the TSS to 3 kilobase pair (kb) downstream of the TTS (for graphical illustration see Figure 22). Moreover, all genes shorter than $600 \mathrm{bp}$ were excluded from the analysis. As an output of DiffBind a normalized count matrix was saved for all individual TSS and GB regions with the respective binding intensity values. Values equal to background were excluded from the analysis. For calculation of the stalling ratio the value at the TSS was divided with the value calculated at the gene body. 


\section{Results}

\subsection{CHD1 depletion impairs MSC and FOB differentiation}

Cellular differentiation is orchestrated by repression and activation of genes mediated by transcription factors and specific signaling pathways. Gene expression changes are often accompanied and maintained by rearrangements in chromatin and histone modifications. In this study the role of the chromatin remodeler CHD1 during the adipocyte and osteoblast differentiation processes was studied. For this purpose multipotent human bone marrow derived mesenchymal stem cells (MSC) (Simonsen et al., 2002) and fetal osteoblast 1.19 cells (FOB) (Harris et al., 1995) were used as a model system. FOB can be differentiated to osteoblasts, and MSC to osteoblasts and adipocytes (Bianco and Robey, 2015; Subramaniam et al., 2002). Further, both cell lines have the potential to form ectopic bone in vivo (Simonsen et al., 2002). Since they have been used previously already for over a decade in various differentiation studies they are well described human differentiation models.

Here the differentiation potential of MSC and FOB was studied prior to and post CHD1 depletion. RNA, protein and cell staining of undifferentiated and differentiated adipocytes or osteoblasts, transfected either with CHD1 siRNA (siCHD1) or as a control with luciferase siRNA (siCon), were analyzed.

As expected, osteoblast differentiated MSC and FOB showed cell-type-associated staining by alkaline phosphatase liver/ bone/ kidney (ALPL) specific activity (Figure $6 \mathrm{~A}$, Figure $7 \mathrm{~A}$ ), whereas in undifferentiated MSC almost no staining was detected (FOB undifferentiated not shown). Interestingly, stronger ALPL staining was observed in CHD1 depleted MSC-derived osteoblasts compared to siCon transfected cells (Figure 6A). While ALPL staining in FOB after five days of differentiation was similar in control transfected compared to CHD1 transfected cells, the ALPL signal intensity was slightly decreased seven days after differentiation in CHD1 reduced conditions (Figure 7A). This indicates a dynamic and cell-type-specific regulation of ALPL in the context of reduced CHD1 levels. Adipocyte-differentiated cells showed an accumulation of lipid droplets compared to undifferentiated control (Figure 6A, panels to the right), which was reduced after CHD1 depletion. This suggests a role of CHD1 in adipocyte differentiation. 
ALPL

A

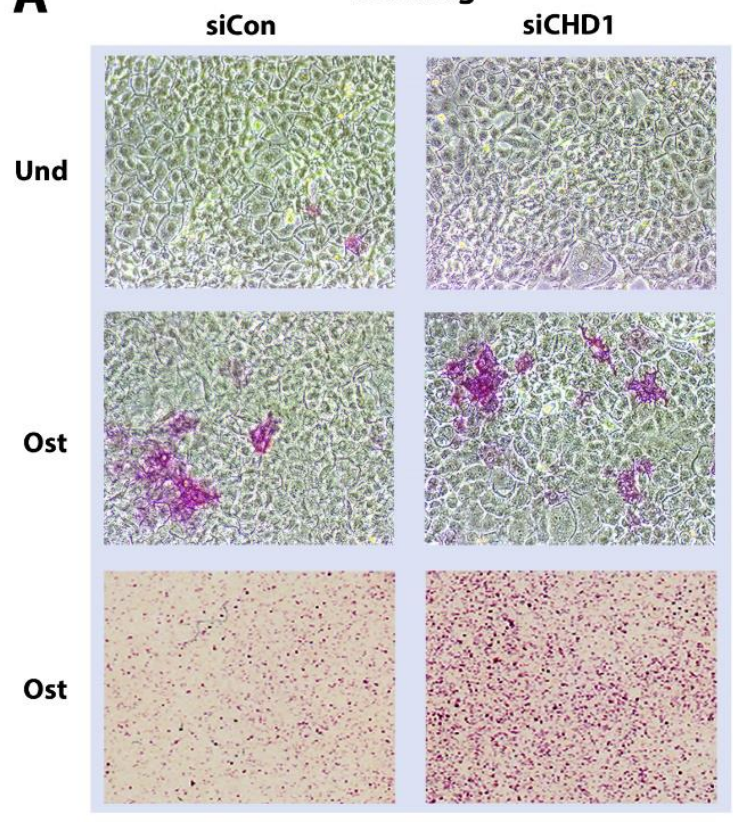

C

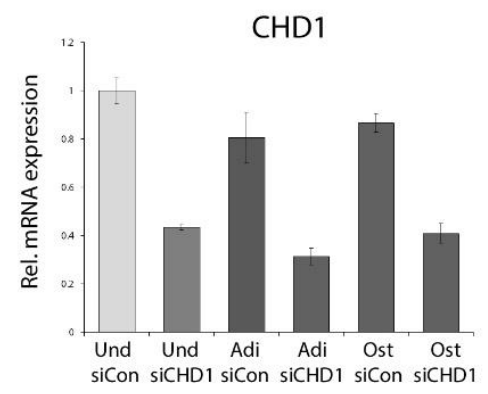

Oil Red 0

staining

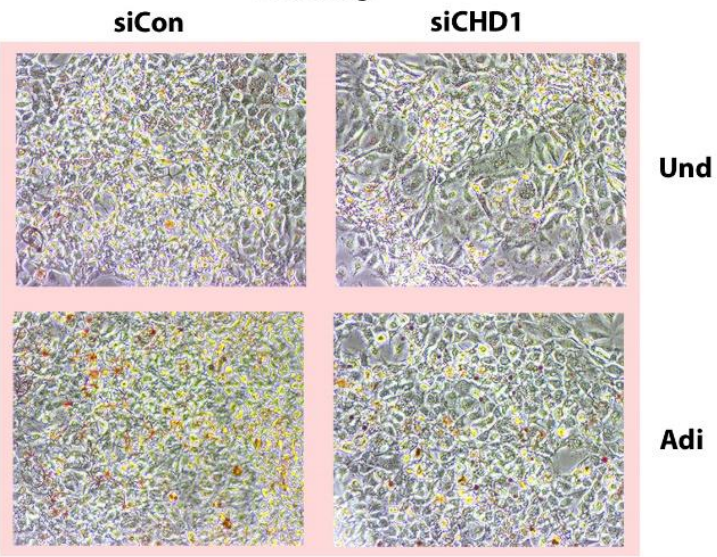

B

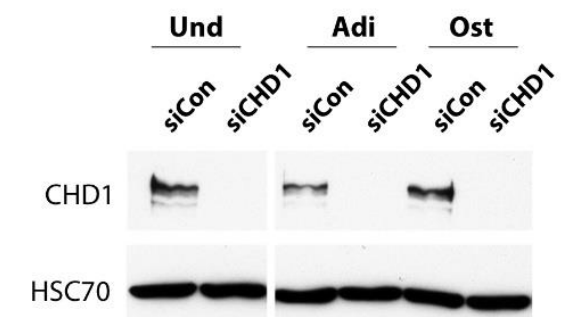

H2Bub1

$\mathrm{H} 2 \mathrm{~B}$

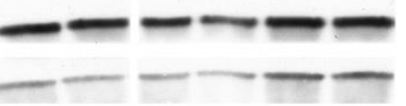

BGLAP

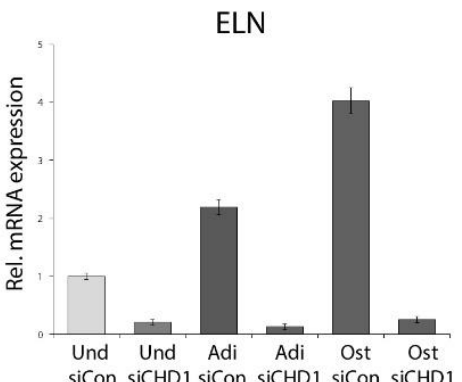

LPL

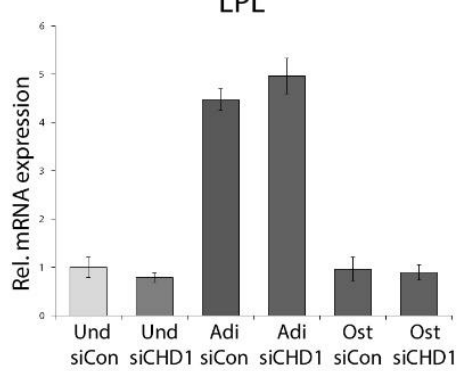

Figure 6: CHD1 depletion alters adipocyte and osteoblast differentiation in MSC. A ALPL activity staining of cells (blue-framed left panels) or of lipid droplets by Oil Red O (red-framed right panels) of osteoblasts (Ost), adipocytes (Adi) or undifferentiated control cells (Und) transfected either with siRNA 
directed against CHD1 (siCHD1) or luciferase (siCon). Cells were differentiated for five days and images taken after staining by bright-field microscopy with 10x (second row panels) or 4x (third row panels) magnification. B Immunodetection of CHD1, mono-ubiquitinated H2B (H2Bub1), heat shock protein family A member 8 (HSC70) or $\mathrm{H} 2 \mathrm{~B}$ with antibodies by Western blot analysis of whole cell protein lysates of differentiated MSC, transfected with siRNA as described in A. C Relative mRNA levels of specific genes (see below) in MSC transfected and differentiated as described in A. The genes analyzed by real time quantitative PCR (qPCR) with gene specific primers and normalized to the 18S rRNA gene expression were the osteoblast associated genes osteocalcin (BGLAP), alkaline phosphatase $(A L P L)$, elastin $(E L N)$ and the adipocyte associated genes peroxisome proliferatoractivated receptor gamma (PPARG), lipoprotein lipase (LPL), periliplin 1 (PLIN1). Values are shown relative to the expression in undifferentiated cell under siCon-transfected conditions. Error bars represent the standard deviation of at least three biological replicates.

A

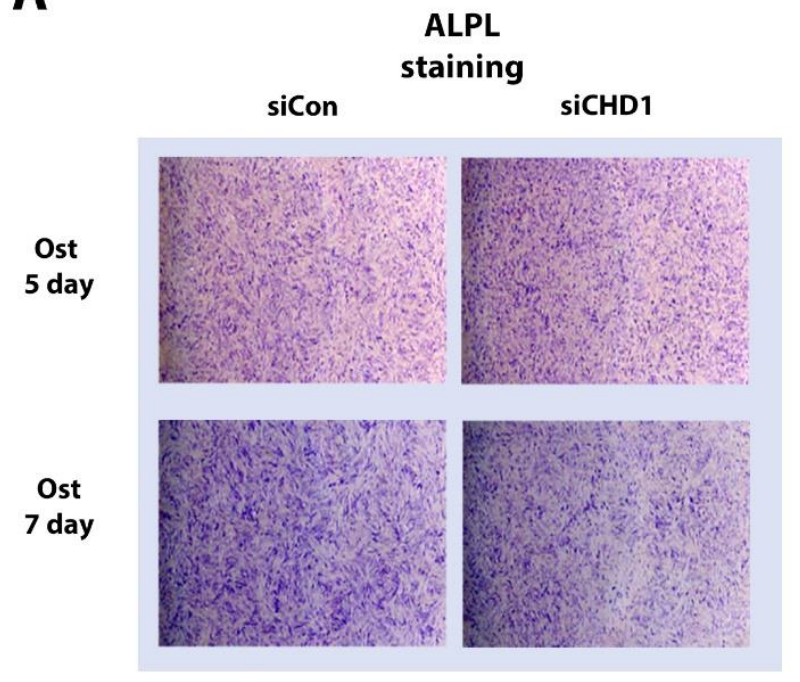

B

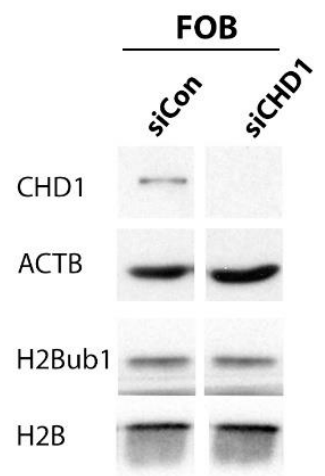

ALPL

BMP4

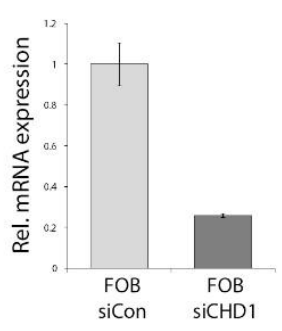

ELN

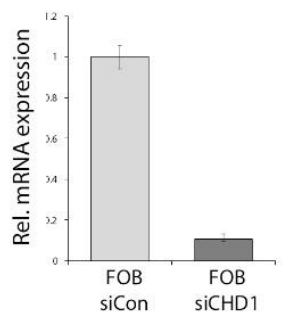

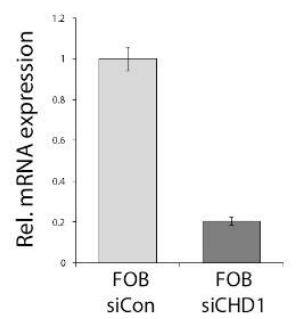

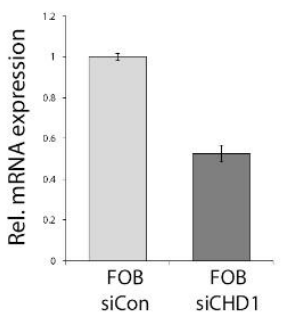

Figure 7: Osteoblast differentiation marker are reduced after CHD1 depletion in FOB.

A Microscopic evaluation of ALPL activity staining of five and seven days' osteoblast differentiated FOB (Ost) transfected with control siRNA against luciferase (siCon) or CHD1 siRNA (siCHD1). Cell culture wells were scanned and representative regions are shown for each condition. B Western blot analysis of whole cell lysates of five days' osteoblast differentiated FOB, transfected with siCon or siCHD1. CHD1, Beta-actin (ACTB), H2Bub1 or H2B were immunodetected with corresponding antibodies by immunoperoxidase. C Relative mRNA levels of osteoblast associated genes ELN, ALPL and bone morphogenic protein 4 (BMP4) in five days differentiated FOB transfected with siCon or siCHD1 as measured by qPCR with gene specific primer and normalized to the $18 S$ rRNA levels. Values are shown relative to the expression in undifferentiated cells under siCon-transfected conditions. Error bars represent the standard deviation of at least three biological replicates. 
The observed differences in the chemical cell staining shown so far were further analyzed by Western blots to initially validate the CHD1 knockdown efficiency on protein level. Additionally, H2Bub1, a PTM histone mark required for adipocyte and osteoblast differentiation (Karpiuk et al., 2012) and shown to be dependent on CHD1, was measured (Lee et al., 2012b). Surprisingly, H2Bub1 was not reduced with decreased CHD1 protein levels neither in MSC nor in FOB when differentiated or undifferentiated (Figure 6B, Figure 7B). This indicates that global H2Bub1 levels are independent from $\mathrm{CHD} 1$ in these cell systems, despite the strongly reduced CHD1 protein levels achieved post siCHD1 transfection. The result of these experiments also indicates that the CHD1-dependent effects on differentiation are probably independent of $\mathrm{H} 2 \mathrm{Bub} 1$-mediated events during differentiation.

\subsection{Cell lineage specific genes are deregulated with reduced CHD1 levels}

To analyze the effect of CHD1 depletion on cell-lineage-specific gene regulation cDNA was analyzed by quantitative real-time PCR (qPCR). Differentiated adipocytes showed a gene induction of lipid storage and glucose-metabolizing proteins perilipin 1 (PLIN1) and lipoprotein lipase ( $L P L)$, as well as the adipogenic transcription factor peroxisome proliferator-activated receptor gamma (PPARG) (Figure 6C). These strong inductions in an adipocyte specific manner support the cell lineage specific differentiation observed previously by Oil Red $O$ staining. Concordant with the reduced Oil Red O staining in CHD1 depleted condition a decreased induction of the adipogenic master regulator PPARG and PLIN1 was observed. LPL was however not regulated arguing against a general inhibition of differentiation-induced genes. Consistent with that, osteoblast differentiated cells showed an increased expression of the early induced, cell lineage specific marker genes BGLAP and ALPL as reported previously (Rickard et al., 1996). In addition, ELN, a gene important for ectopic bone formation and expressed during early osteoblast differentiation (Larsen et al., 2010; Twine et al., 2014), was induced, too. Two of the osteoblastic marker genes $A L P L$ and BGLAP were higher expressed after CHD1 depletion compared to control transfected cells, whereas $E L N$ was strongly repressed under all conditions (Figure $6 \mathrm{C}$ ). This together this strongly argues for a CHD1 specific regulation on cell differentiation. In FOB $A L P L, B M P 4$ and $E L N$ were repressed in expression upon reduction of $\mathrm{CHD} 1$ levels (Figure $7 \mathrm{C}$ ). However, BGLAP, an early marker for 
differentiation, was not expressed, which highlights potential differences in cell lineage commitment between the MSC and FOB.

In sum MSC and FOB were lineage specifically differentiated as evident by the expression of cell type specific marker genes and characteristic phenotypic staining. CHD1 depletion caused an altered differentiation pattern compared to control siRNA transfected cells. The osteoblast specific marker genes ALPL and BGLAP were higher expressed and showed concordantly increased ALPL staining. This strongly suggests that the cells entered the osteoblast lineage, but possibly in a slightly restricted manner when taking the observed repression of ELN as a marker into account. In contrast, in FOB osteoblast associated genes were inhibited by CHD1 depletion and showed minor reduction of ALPL staining at day seven. These observed differences compared to MSC might be caused by different stages of cell lineage commitment, a speculation supported by the observed lack of BGLAP expression.

Adipocyte differentiation was inhibited upon CHD1 depletion as deduced from decreased lipid body formation and PPARG repression. Although cell type change is often initiated and characterized by single factors, the full differentiation process is multifactorial with timely orchestration of different signaling pathways. To further address the question of global gene regulation during differentiation in CHD1 depleted conditions the transcriptome was analyzed by deep RNA sequencing (RNAseq).

\subsection{Transcriptomic differences in MSC after CHD1 depletion and differentiation}

Transcriptome-wide differential expression analysis helps to distinguish and characterize cell states. Here we sought to analyze differences of gene regulatory networks and pathways, which could further elucidate the differentiation effects observed in the previous part. Therefore, poly-adenylated mRNA was isolated from undifferentiated and seven-days-differentiated MSC and FOB, transfected with either siCon or siCHD1.

Genome-wide differences in mRNA expression of the different conditions in MSC were first measured by principal component analysis (PCA). Therefore the variation between all expressed genes were reduced to two dimensions, the principal component 1 and 2 (PC1, PC2). Each condition was plotted in biological duplicates, as used in the RNA-seq study. The PCA plot showed high similarity between the 
biological duplicates for each condition (Figure 8). Further we observed that the osteoblast and adipocyte differentiated transcriptomes strongly shifted from the undifferentiated state on PC1, but only the osteoblast condition shifted also on PC2, which indicates differences between all three control transcriptomes. The transcriptome of CHD1 depleted samples shifted mainly on the PC2 axis when compared to their control condition. Interestingly however was that the CHD1 depleted osteoblast condition was shifted apart less from undifferentiated control than the osteoblast control sample was. This implies a decrease in the global change of transcription during osteoblast differentiation when CHD1 protein levels were reduced. However, the smallest change introduced by CHD1 depletion to its relative control was observed in the undifferentiated cells, which suggests a greater influence of CHD1 on gene expression during differentiation than in an undifferentiated condition. Together, this shows that different transcriptome wide changes were introduced with differentiation and CHD1 depletion, however, interestingly, caused reduced levels of CHD1 during osteoblast differentiation decreased changes in the transcription

profile.

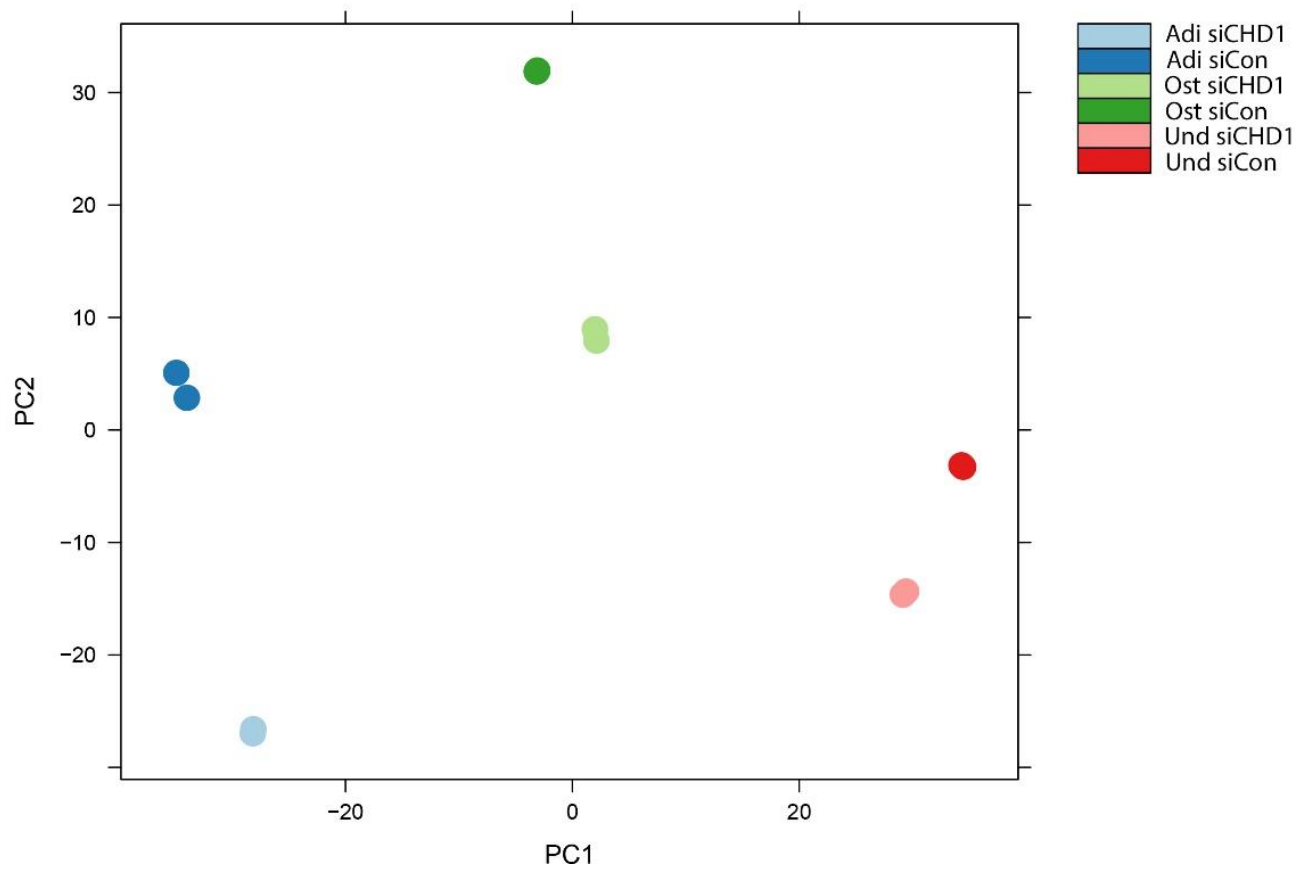

Figure 8: Differentiation and CHD1 depletion causes broad transcriptomic changes. The principal component analysis (PCA) plot represents the transcriptome of all genes in undifferentiated (Und) and 5 days differentiated adipocyte (Adi) or osteoblast (Ost) cells transfected either with siCHD1 or siCon. Each dot shows one of two RNA-seq samples of the respective condition indicated by the color code shown top right. The samples were calculated based on their normalized gene expression counts for all human genes and plotted on the principal component 1 (PC1) and 2 (PC2). 


\subsection{CHD1 depletion attenuates regulation of differentiation regulated genes}

Differentiation specific gene sets in MSC and FOB were grouped by thresholds of plus or minus $0.5 \log _{2}$ fold change values $\left(+/-0.5 \log _{2} \mathrm{FC}\right)$, with a multiple testing adjusted probability value below 0.05 (p-adj. < 0.05). With these thresholds 2,478 genes were repressed and 1,986 genes were activated during adipocyte differentiation, whereas in osteoblast differentiation a lower number of 1,379 genes showed increased and 1,408 genes showed decreased expression compared to control (Figure 9A, B, left panel). This shows greater gene expression changes in adipocyte differentiated cells than in osteoblasts, which might indicate a closer osteoblast cell fate commitment of the bone marrow derived MSC (Simonsen et al., 2002). During FOB osteoblast differentiation 4,012 genes were increased in their expression and 4,869 were decreased compared to undifferentiated control condition (Figure 9C, left panel).

Next the impact of CHD1 on the genes regulated during differentiation was investigated. Genes induced during adipocyte and osteoblast differentiation were reduced in their activation and repressed genes during differentiation were mitigated in their silencing (Figure 9A, B, C, right panel). Thus CHD1 depletion mainly attenuates the effect on gene regulation taking place during differentiation. To further identify the significant differentially expressed genes after CHD1 depletion the groups selected above were filtered with similar thresholds to the differentiated SiCHD1treated condition $\left(+/-0.5 \log _{2} F C, p\right.$-adj. < 0.05). This showed that the majority of significant $\mathrm{CHD} 1$ regulated genes during differentiation were indeed regulated in an opposite manner compared to the control differentiation (Figure 9A, B, C, pie charts). Interestingly, this effect was stronger during osteoblast differentiation than adipocyte differentiation. In particular, out of all 1,379 genes activated by osteogenesis were 550 significantly reduced in their induction, but only 155 were further increased when CHD1 protein levels were reduced (Figure 9B, pie chart). Furthermore, in total 579 genes in FOB were repressed after CHD1 depletion (not shown). Strikingly, 472 of these genes were also regulated during the differentiation process (Figure 9C, blue parts of pie charts). This indicates a major effect of CHD1 especially on the differentiation regulated genes in FOB. In MSC, approximately $40 \%$ of all repressed genes with CHD1 knockdown were also regulated during osteoblast differentiation which argues for a broader regulation, not only on differentiation specific genes. 
In sum, the transcriptome of MSC and FOB was broadly changed in over thousands of genes within 5 days of differentiation. CHD1 depletion affected the gene expression by weakening the regulatory impact of the differentiation program, most evident in osteoblast differentiation. This attenuation of the differentiation effect was also observed by the decreased shift shown in the PCA plot for CHD1 depleted osteoblasts.

A
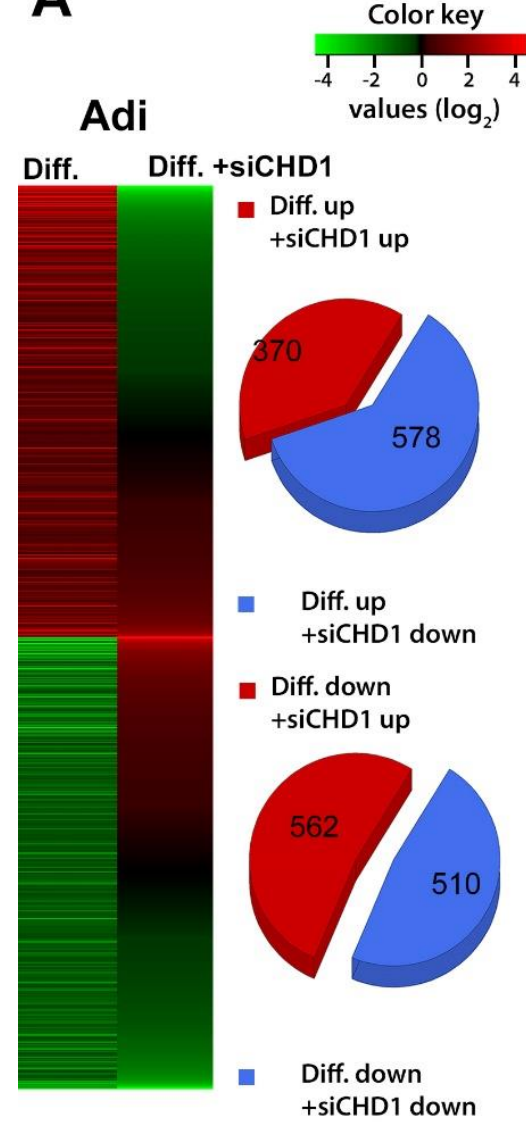

B

Ost

Diff. Diff. +siCHD1

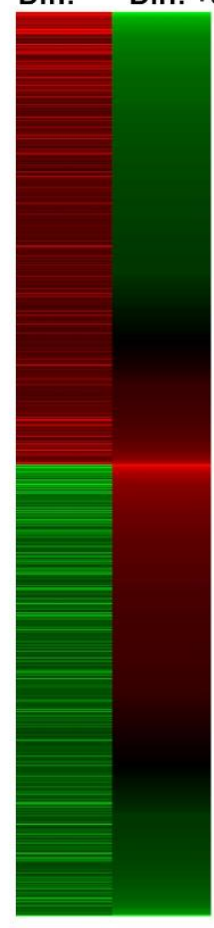

- Diff. up + siCHD1 up

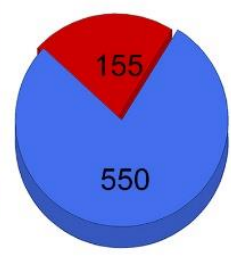

- Diff. up +siCHD1 down

- Diff. down + siCHD1 up

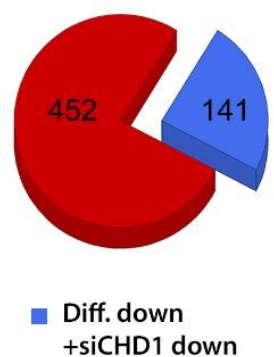

C

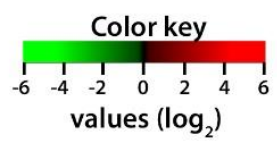

FOB

Diff. Diff. +sicHD1

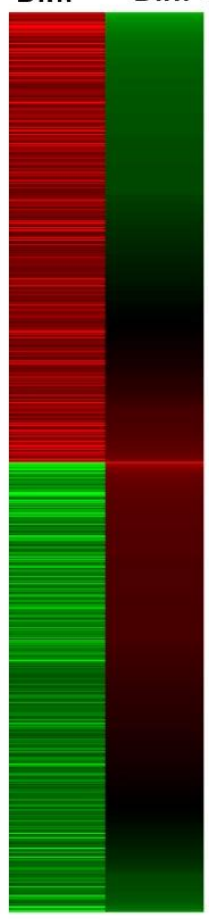

- Diff. up + siCHD1 up

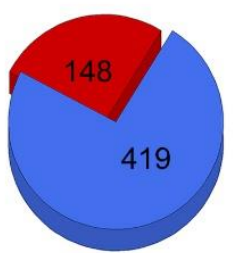

Diff. up +siCHD1 down

- Diff. down + siCHD1 up

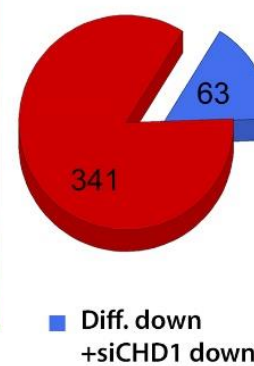

Figure 9: Genes regulated during differentiation are attenuated upon CHD1 depletion. A Heat map represents the $\log _{2}$ fold changes in mRNA expression during adipocyte differentiation (Adi, Diff.) compared to relative changes after CHD1 depletion (Diff. + siCHD1). Genes were defined by differentially expression of undifferentiated siRNA-Control condition compared to adipocyte differentiated siRNA-Control condition (left panel). Further, gene expression changes between adipocyte control (Diff.) and adipocyte CHD1 depletion conditions are shown (right panel). Differentiation genes were filtered by values above or below $0.5 \log _{2}$ fold changes $\left(+/-0.5 \log _{2} \mathrm{FC}\right)$ and an adjusted probability value below 0.05 ( $p$-adj. < 0.05 ). In total 1,986 genes higher expressed (upper half) and 2,478 repressed genes (lower half) during adipocyte differentiation are plotted. The $\log _{2} \mathrm{FC}$ values of the heat map are shown in the color key. Pie charts depict the numbers of significantly repressed (blue) or activated (red) differentiation genes upon CHD1 depletion with thresholds described above. B Heat map and pie charts represent genes regulated during MSC osteoblast differentiation and the relative regulation by siRNA mediated CHD1 depletion. In total 1,379 genes were higher (upper part) and 1,408 were lower (lower part) expressed during osteoblast differentiation. For further description see A. C Heat map and pie charts show genes regulated during FOB osteoblast differentiation and the relative regulation by siRNA mediated CHD1 depletion. In total 4,012 genes 
were higher (upper part) and 4,869 lower (lower part) expressed during osteoblast differentiation. For further description see $A$.

\subsection{Low transcribed genes require CHD1 for their activation during differentiation}

As CHD1 was necessary for the gene regulation during differentiation we sought to further investigate the transcription levels of these CHD1 regulated genes (Figure 9, pie charts). Therefore, the normalized transcription counts were plotted twodimensionally for differentiated and undifferentiated cells in control and CHD1 depleted condition.

The selected genes showed a broad distribution of expression levels in undifferentiated and differentiated conditions (Figure 10A, B, C). This implies that CHD1 differentiation affected genes were distributed over a wide range of transcription values. To clarify the data, a regression curve was plotted through the gene expression points of undifferentiated and differentiated control conditions. Interestingly, all investigated gene subsets showed particular strong changes during differentiation in the lowly expressed genes (Figure 10, black lines). Particularly, lowly transcribed genes in undifferentiated state were mainly activated during differentiation and not further repressed. Strikingly, these genes were changed less prominent in CHD1 depleted condition and closer resembled the undifferentiated control expression levels (Figure 10, red lines). This suggests that the lowly expressed genes required CHD1 for their full activation.

Next, we sought to quantify the changes in gene expression introduced by CHD1 depletion especially during osteoblast differentiation. The initial data of the PCA plot showed a reduced transcriptomic shift during osteoblast differentiation relative to undifferentiated control when CHD1 was depleted. For further analysis, the Pearson correlation coefficient $(R)$ for the data represented in the scatter plot was calculated. It describes the similarity of data values in a range from -1 (low) to 1 (high). We could observe an increase of the correlation coefficient between undifferentiated and siCHD1 treated osteoblasts compared siCon treated osteoblasts. The increase was similar in FOB and MSC from 0.72 to 0.78 and 0.78 to 0.84 , respectively (Figure 10B, C). Interestingly, the regression curve suggests that the most affected genes were low expressed ones. This argues for reduced changes in gene expression during osteoblast differentiation when CHD1 is depleted. Surprisingly, this effect was not observed in adipogenesis. All genes were further changed in their expression relative 
to undifferentiated control or the regulation pattern was more heterogeneous which might overlay the effect on induced genes while adipocyte differentiation. This together indicates that the regulatory effects in osteoblast and adipocytes could be different. Further, CHD1 was required to introduce changes in gene expression during osteoblast differentiation and decreased CHD1 protein levels rather maintained the undifferentiated expression profile than further changing it.

A
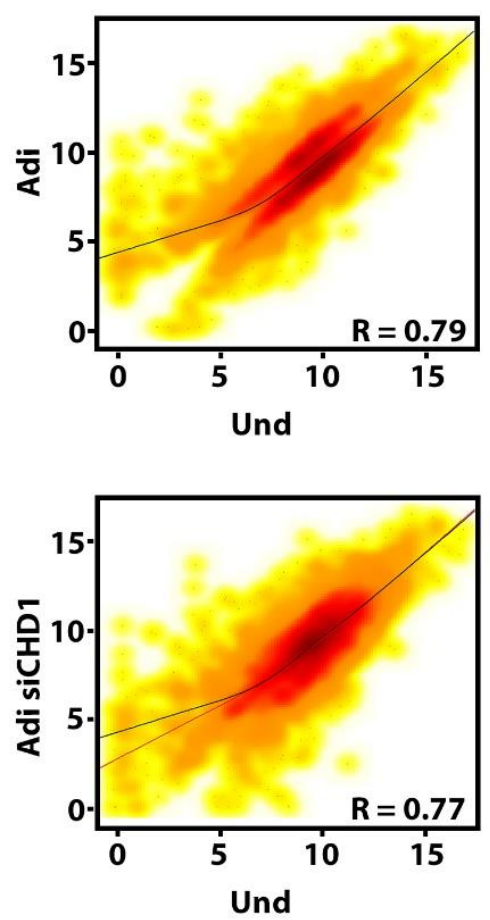

B
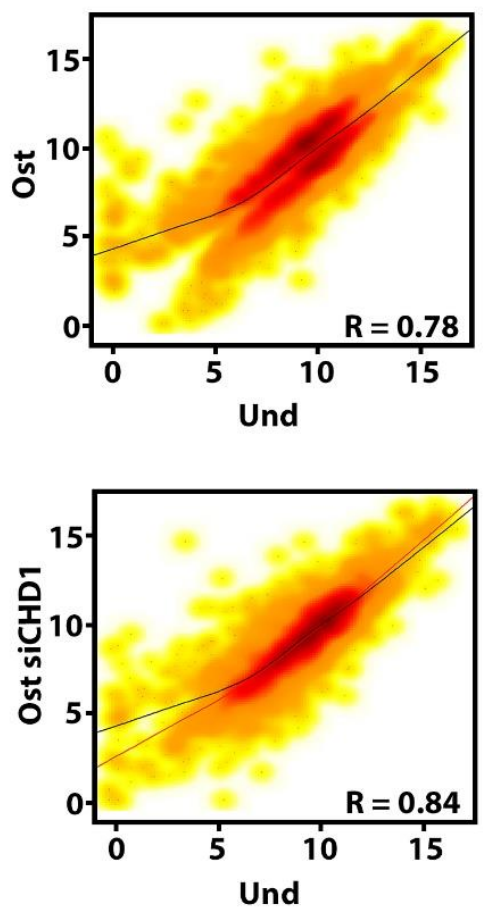

C
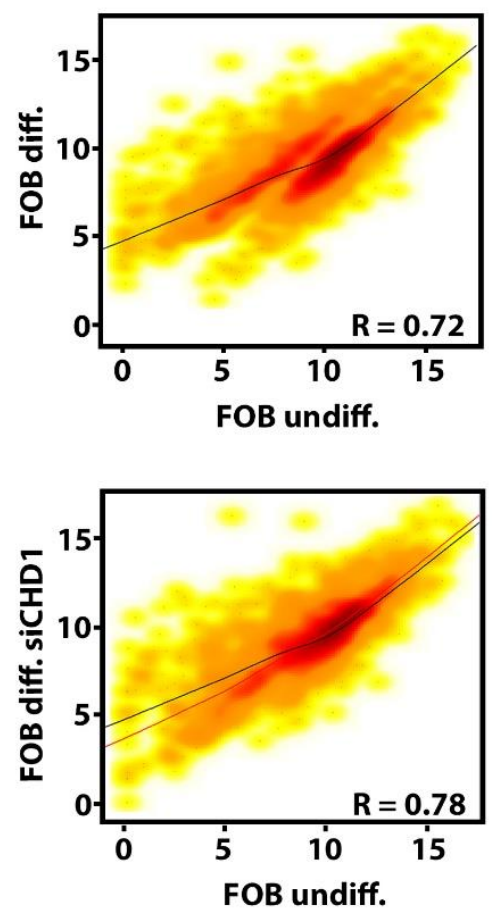

Figure 10: CHD1 is necessary for induced changes in gene expression during osteogenesis. A Scatter plots show normalized gene expression values between undifferentiated and adipocyte differentiated condition with siCon (Adi) or siCHD1 treatment (siCHD1) in MSC. Genes were selected by significant changes of expression during differentiation ( $\mathrm{p}$-adj. $<0.05,+$ or $-0.5 \log _{2} \mathrm{FC}$ ) and significant regulation by siRNA mediated CHD1 depletion ( $p$-adj. $<0.05,+/-0.5 \log _{2} \mathrm{FC}$ ). A regression curve is shown in black for control and in red for the siCHD1 condition. The Pearson correlation coefficient $(R)$ is shown at the bottom right of each plot. The color of the plot indicates the gene densities from low (yellow) to high (red). In total, 2,020 genes were plotted similar. B Scatter plots show normalized gene expression values of undifferentiated and osteoblast differentiated condition with siCon (Ost) or siCHD1 treatment (siCHD1) in MSC. Gene selection, thresholds and figure description were used as described in A. In total 1,298 genes were plotted. C Scatter plots show normalized gene expression values between undifferentiated and osteoblast differentiated condition with siCon (Ost) or siCHD1 treatment (siCHD1) in FOB. For further gene selection, thresholds and figure description see A. In total, 971 genes were plotted similar as described in Figure $9 \mathrm{C}$ pie charts.

\subsection{CHD1 regulated genes are enriched in osteoblastic gene ontology terms}

Transcriptome-wide data can be used to analyze regulatory networks or biological functions for a better characterization of cellular states. Therefore, we used all 
deregulated genes by CHD1 depletion during the differentiation process in MSC and FOB as described previously (Figure 9A, B, C). These groups were analyzed for their enrichment in Gene Ontology (GO) terms of biological processes and cellular compartments defined by the GO Consortium and built-in the Database for Annotation, Visualization and Integrated Discovery (DAVID) software.

The highest annotation cluster in MSC as well as FOB showed GO terms associated with the extracellular region and extracellular matrix (ECM) (Table 1Table 2Table 3). Both cellular compartments undergo strong changes during differentiation, which are necessary for cell type specific functions like deposition of collagen before matrix mineralization in osteoblasts or structural support of the membrane previous to storage of lipids (Damsky, 1999; Mariman and Wang, 2010). Changes in the ECM during differentiation were also observed in the microscopic images for cell type specific staining (Figure 6A). Besides the ECM organization, the extracellular region term involves secreted protein like BGLAP or ELN. These genes were previously shown to be strongly deregulated by CHD1 depletion (Figure 6C, Figure 7C).

Additionally, the top annotation clusters further selected terms showed impairment of important supportive biological processes for adipocyte and bone formation such as angiogenesis and responses to endogenous stimuli (Eshkar-Oren et al., 2009; Ledoux et al., 2008). This implies that the crosstalk between osteoblast or MSC with vascularizing tissue might be perturbed which could influence proper bone formation. Further, signaling pathways stimulated by differentiation medium or in a paracrine manner required $\mathrm{CHD} 1$ for proper regulation. Deregulated signaling pathways maybe involved in indirect gene regulation independent of $\mathrm{CHD} 1$.

Strikingly, bone related $\mathrm{GO}$ terms were significantly enriched within the deregulated gene groups of osteoblast differentiated MSC and FOB (Table 2, Table 3 bold). We compared the genes of the top term "skeletal system development" with the fold changes in gene expression identified by RNA-seq. Intriguingly, it showed that 44 out of 56 deregulated genes in MSC and 23 out of 30 in FOB were repressed in their activation during osteogenesis when CHD1 protein levels were low. This argues for a role of CHD1 in the activation of skeletal development associated genes. Summarized, biological processes and cellular compartments which are important for bone formation required CHD1 for normal regulation during early osteogenesis. Furthermore, terms related to bone formation also showed perturbed regulation by CHD1 depletion which might affect normal bone development. 
Table 1: Gene Ontology terms of CHD1 dependent genes during adipocyte MSC differentiation. The table lists gene ontology (GO) terms of deregulated genes after CHD1 depletion during adipocyte differentiation of MSC. Genes were selected by thresholds described in Figure 9A (pie chart) and analyzed for enriched GO terms with the Database for Annotation, Visualization and Integrated Discovery (DAVID) software. In total 2,020 genes were used as an input with the all human genes set as background. Results were sorted by the enrichment score or their false discovery rate (FDR). The gene number (Count) within the respective GO term (Term) and probability values calculated by the Benjamini-Hochberg procedure (Benjamini) are shown on top of the table.

\begin{tabular}{|c|c|c|c|c|}
\hline $\begin{array}{c}\text { Annotation Cluster 1 } \\
\text { Category }\end{array}$ & Enrichment Score: 9.90 & & \\
\hline Cellular Compartment & Extracellular region part & 168 & $5.37 \mathrm{E}-11$ & $1.45 \mathrm{E}-10$ \\
\hline Cellular Compartment & Extracellular region & 282 & $3.21 \mathrm{E}-08$ & $2.60 \mathrm{E}-07$ \\
\hline Cellular Compartment & Extracellular space & 112 & $9.87 \mathrm{E}-06$ & $1.60 \mathrm{E}-04$ \\
\hline
\end{tabular}

\begin{tabular}{|c|c|c|c|c|}
\hline Annotation Cluster 2 & Enrichment Score: 8.97 & \multicolumn{1}{c|}{ Count } & \multicolumn{1}{c|}{ Benjamini } & FDR \\
\hline Category & Term & 73 & $9.35 \mathrm{E}-08$ & $1.01 \mathrm{E}-06$ \\
\hline Cellular Compartment & Extracellular matrix & 68 & $2.57 \mathrm{E}-07$ & $3.46 \mathrm{E}-06$ \\
\hline Cellular Compartment & Proteinaceous extracellular matrix & 29 & $3.39 \mathrm{E}-04$ & $1.19 \mathrm{E}-02$ \\
\hline Cellular Compartment & Extracellular matrix part & &
\end{tabular}

\begin{tabular}{|c|c|c|c|c|}
\hline Selected terms & & & & \\
\hline Category & Term & Count & Benjamini & FDR \\
\hline Biological Process & Vasculature development & 50 & 1.17E-03 & 1.05E-02 \\
\hline Biological Process & Blood vessel morphogenesis & 43 & 1.87E-03 & 2.94E-02 \\
\hline Biological Process & Angiogenesis & 30 & 2.02E-02 & 7.59E-01 \\
\hline Biological Process & Response to endogenous stimulus & 71 & 1.13E-03 & 1.16E-02 \\
\hline Biological Process & Response to hormone stimulus & 63 & 3.97E-03 & 7.85E-02 \\
\hline Cellular Compartment & Plasma membrane & 441 & $8.78 \mathrm{E}-04$ & $3.55 E-02$ \\
\hline
\end{tabular}


Table 2: Gene Ontology terms of CHD1 dependent genes during osteoblast differentiation in MSC.

The table lists gene ontology (GO) terms of deregulated genes after CHD1 depletion during osteoblast differentiation of MSC. Genes were selected by thresholds described in Figure 9B (pie chart) and analyzed for enriched GO terms with the Database for Annotation, Visualization and Integrated Discovery (DAVID) software. In total 1,298 genes were used as an input with the all human genes set as background. For further description of the table see Table 1.

\begin{tabular}{|c|c|c|c|c|}
\hline Annotation Cluster 1 & Enrichment Score: 11.66 & & & \\
\hline Category & Term & Count & Benjamini & FDR \\
\hline Cellular Compartment & Extracellular region part & 132 & 4.87E-14 & $1.55 \mathrm{E}-13$ \\
\hline Cellular Compartment & Extracellular region & 205 & 1.78E-09 & $1.72 \mathrm{E}-08$ \\
\hline Cellular Compartment & Extracellular space & 85 & $6.04 \mathrm{E}-07$ & 1.75E-05 \\
\hline
\end{tabular}

\begin{tabular}{|c|c|c|c|c|}
\hline Annotation Cluster 2 & Enrichment Score: 10.63 & & & \\
\hline Category & Term & Count & Benjamini & FDR \\
\hline Cellular Compartment & Extracellular matrix & 61 & $4.76 \mathrm{E}-10$ & 3.06E-09 \\
\hline Cellular Compartment & Proteinaceous extracellular matrix & 55 & 1.03E-08 & 1.32E-07 \\
\hline Cellular Compartment & Extracellular matrix part & 22 & $6.48 E-04$ & 2.92E-02 \\
\hline
\end{tabular}

\begin{tabular}{|c|c|c|c|c|}
\hline Annotation Cluster 7 & Enrichment Score: 6.02 & \multicolumn{2}{c|}{} & \\
\hline Category & Term & Count & Benjamini & FDR \\
\hline Biological Process & Skeletal system development & 56 & $4.01 \mathrm{E}-08$ & $6.31 \mathrm{E}-08$ \\
\hline Biological Process & Bone development & 24 & $5.18 \mathrm{E}-04$ & $8.43 \mathrm{E}-03$ \\
\hline Biological Process & Ossification & 22 & $1.40 \mathrm{E}-03$ & $3.16 \mathrm{E}-02$ \\
\hline Biological Process & Osteoblast differentiation & 11 & $1.33 \mathrm{E}-02$ & $5.67 \mathrm{E}-01$ \\
\hline
\end{tabular}

\begin{tabular}{|c|c|c|c|c|}
\hline Selected terms & & & & \\
\hline Category & Term & Count & Benjamini & FDR \\
\hline Biological Process & $\begin{array}{c}\text { Response to endogenous } \\
\text { stimulus }\end{array}$ & 59 & $3.51 \mathrm{E}-06$ & $2.76 \mathrm{E}-05$ \\
\hline Biological Process & Response to hormone stimulus & 53 & 2.22E-05 & 2.21E-04 \\
\hline Biological Process & Vasculature development & 36 & $1.51 \mathrm{E}-03$ & 3.63E-02 \\
\hline Biological Process & Blood vessel development & 35 & $1.86 \mathrm{E}-03$ & 5.26E-02 \\
\hline Biological Process & Angiogenesis & 22 & $2.43 E-02$ & $1.26 \mathrm{E}+00$ \\
\hline Cellular Compartment & Plasma membrane & 323 & 3.69E-07 & 9.51E-06 \\
\hline
\end{tabular}


Table 3: Gene Ontology terms of CHD1 dependent genes during osteoblast differentiation in FOB. The table lists gene ontology (GO) terms of deregulated genes after CHD1 depletion during osteoblast differentiation of FOB. Genes were selected by thresholds described in Figure 9C (pie chart) and analyzed for enriched GO terms with the Database for Annotation, Visualization and Integrated Discovery (DAVID) software. In total 971 genes were used as an input with the all human genes set as background. For further description of the table see Table 1.

\begin{tabular}{|c|c|c|c|c|}
\hline Annotation Cluster 1 & Enrichment Score: 8.44 & & & \\
\hline Category & Term & Count & \multicolumn{1}{c|}{ Benjamini } & FDR \\
\hline Cellular Compartment & Extracellular matrix & 46 & $2.25 \mathrm{E}-07$ & $1.44 \mathrm{E}-06$ \\
\hline Cellular Compartment & Proteinaceous extracellular matrix & 43 & $4.55 \mathrm{E}-07$ & $4.37 \mathrm{E}-06$ \\
\hline Cellular Compartment & Extracellular matrix part & 18 & $3.26 \mathrm{E}-03$ & $6.26 \mathrm{E}-02$ \\
\hline
\end{tabular}

\begin{tabular}{|c|c|c|c|c|}
\hline Annotation Cluster 2 & Enrichment Score: 7.74 & & & \\
\hline Category & Term & Count & Benjamini & FDR \\
\hline Cellular Compartment & Extracellular region part & 98 & $5.47 \mathrm{E}-10$ & $1.75 \mathrm{E}-09$ \\
\hline Cellular Compartment & Extracellular region & 141 & $2.37 \mathrm{E}-04$ & $3.03 \mathrm{E}-03$ \\
\hline Cellular Compartment & Extracellular space & 62 & $2.09 \mathrm{E}-04$ & $3.34 \mathrm{E}-03$ \\
\hline
\end{tabular}

\begin{tabular}{|c|c|c|c|c|}
\hline $\begin{array}{c}\text { Selected terms } \\
\text { Category }\end{array}$ & \multicolumn{1}{c|}{ Term } & Count & Benjamini & FDR \\
\hline Biological Process & Skeletal system development & 30 & $\mathbf{4 . 6 4 E}-02$ & $\mathbf{8 . 1 5 E}-01$ \\
\hline & & & & \\
\hline Biological Process & Blood vessel development & 26 & $2.93 \mathrm{E}-02$ & $3.53 \mathrm{E}-01$ \\
\hline Biological Process & Vasculature development & 26 & $3.40 \mathrm{E}-02$ & $5.12 \mathrm{E}-01$ \\
\hline & & & & \\
\hline Biological Process & Response to organic substance & 62 & $1.19 \mathrm{E}-03$ & $7.09 \mathrm{E}-03$ \\
\hline Biological Process & Response to endogenous stimulus & 36 & $3.55 \mathrm{E}-02$ & $5.77 \mathrm{E}-01$ \\
\hline Biological Process & Response to hormone stimulus & 33 & $4.69 \mathrm{E}-02$ & $8.80 \mathrm{E}-01$ \\
\hline & & & & \\
\hline Cellular Compartment & Intrinsic to plasma membrane & 86 & $1.30 \mathrm{E}-02$ & $3.76 \mathrm{E}-01$ \\
\hline Cellular Compartment & Integral to plasma membrane & 83 & $2.31 \mathrm{E}-02$ & $7.45 \mathrm{E}-01$ \\
\hline
\end{tabular}

\subsection{Validation of single genes identified by RNA-seq with individual CHD1- SiRNAs}

To confirm CHD1 specific gene regulation and to exclude off-target effects by the CHD1 siRNA pool, single siRNAs of the pool were used to validate the gene regulation observed in the RNA-seq experiments. MSC and FOB were transfected with CHD1 targeting siRNA \#1 and \#2 and differentiated for 5 days.

Both single siRNAs \#1 and \#2 showed strong depletion of CHD1 protein and CHD1 mRNA in MSC and FOB (Figure 11A, B, C, D). In addition, genes listed in the "skeletal development" GO term in MSC like endothelin 1 (EDN1), SIX homeobox 1 (SIX1), periostin (POSTN) or dual specificity phosphatase 1 (DUSP1) were confirmed 
to be regulated by both single siRNAs in similar efficiency to the siCHD1 pool observed in the RNA-seq. Also genes analyzed in the previous experiments showed similar regulation by single siRNAs in MSC ( $A L P L, E L M$ ) and FOB (bone morphogenetic protein 4 (BMP4), ELN, POSTM) (compare Figure 11B, D to Figure $6 \mathrm{C}$, Figure $7 \mathrm{C}$ ). Additionally, osteoblastic marker genes were repressed by $\mathrm{CHD} 1$ depletion in FOB, like the Wnt-signaling factor AXIN2 or osteoblast differentiation associated gene connective tissue growth factor (CTGF). This together indicates that the genes regulated by the siCHD1 pool are unlikely off-target effects caused by the siRNA pool. 
A

B
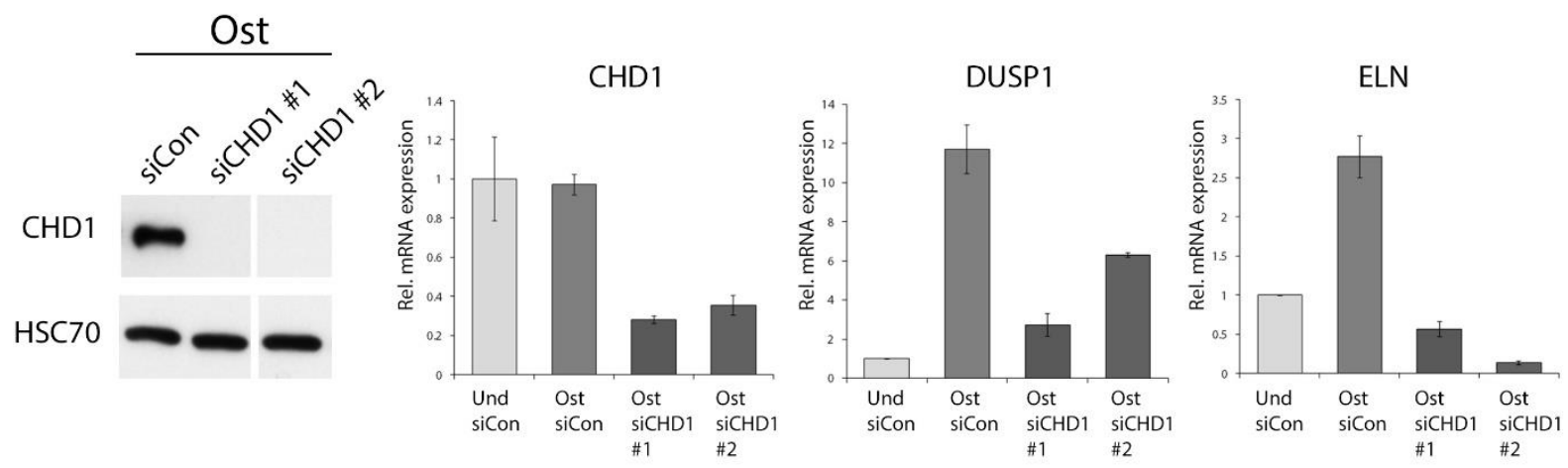

ALPL

POSTN

SIX1

EDN1
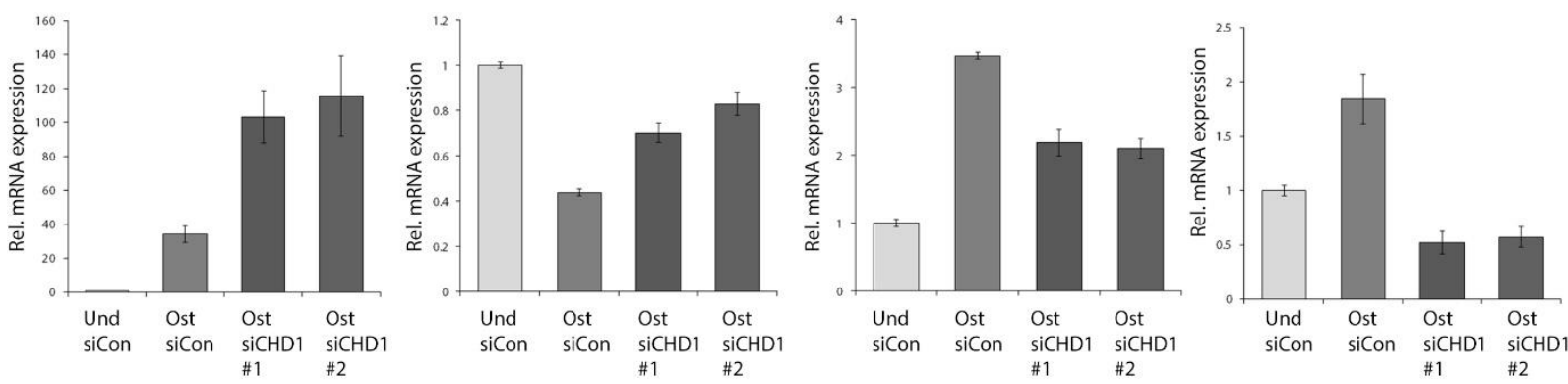

C

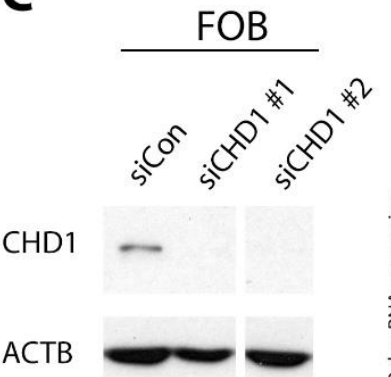

D

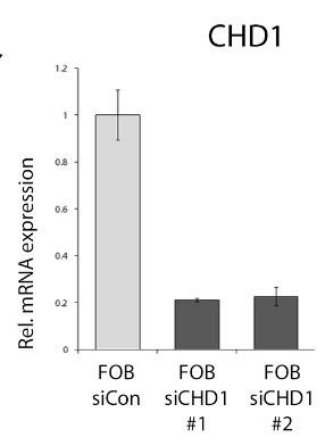

BMP4

ELN
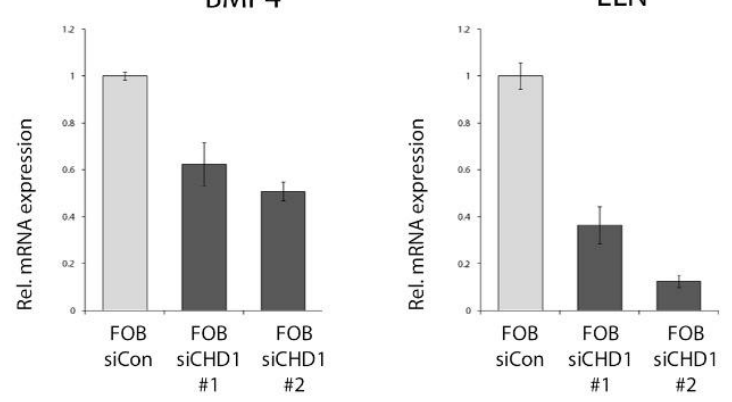

COL11A1

CTGF
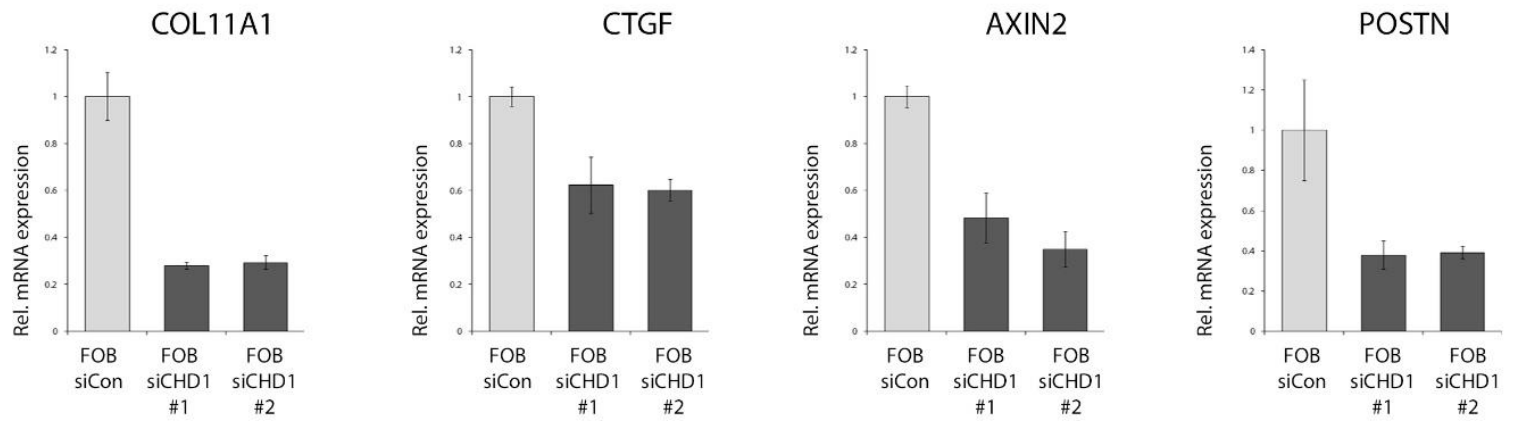

Figure 11: Individual CHD1-siRNAs validate CHD1-specific gene regulation observed by RNAseq. A Immunodetection of CHD1 and HSC70 by Western blot analysis of whole cell lysates of 5 days' osteoblast differentiated MSC, transfected with siRNA directed against luciferase (siCon) or individual siRNA \#1 and \#2 against CHD1. B qPCR analysis of RNA from cells described in A by using gene specific primer against $C H D 1$, alkaline phosphatase, liver/bone/kidney $(A L P L)$, elastin $(E L M)$, dual specificity phosphatase 1 (DUSP1), periostin (POSTM), SIX homeobox 1 (SIX1) and Endothelin 1 (EDN1). Gene expression was normalized to the RNA level of the gene encoding the ribosomal protein, large, PO (RPLPO) and compared relative to that of the osteoblast differentiated siCon condition. Error bars represent the standard deviation of at least 3 biological replicates. C Immunodetection of CHD1 and ACTB by Western blots of whole cell lysates of 5 days' osteoblast 
differentiated FOB, transfected with siRNA directed against luciferase (siCon) or individual siRNA \#1 and \#2 against CHD1. D qPCR analysis of cDNA from cells described in $C$ by using gene specific primer against CHD1, POSTN, bone morphogenetic protein 4 (BMP4), ELN, connective tissue growth factor (CTGF), collagen, type XI, alpha 1 (COL11A1) and AXIN2. Gene expression was normalized to that of the RPLPO gene and compared relative to the osteoblast differentiated siCon condition. Error bars represent the standard deviation of at least 3 biological replicates.

\subsection{Heterozygous CHD1 knockout mice show an abnormal bone phenotype}

As CHD1 depletion caused deregulated differentiation pattern in MSC and FOB which were connected to $\mathrm{GO}$ terms of skeletal development we were curious to see if CHD1 would also impact bone formation in vivo. The international mouse phenotyping consortium (IMPC) describes phenotypes for a constitutive homozygous and heterozygous CHD1 knockout mouse model. The homozygous CHD1 knockout mouse is embryonic lethal before day six, but the heterozygous model is viable with a described phenotype. Among other phenotypes the mice showed significant abnormal skull shape and teeth morphology, which is often associated with defects in bone formation by an interplay between osteoblasts and osteoclasts (Gama et al., 2015). Strikingly however was that the mice showed a significant decrease in body length, bone mineral density and bone mineral content (Figure 12A). This clearly indicates defects in the bone in a CHD1 heterozygous genotype. The phenotype on adipocyte-associated tissues were less clear. CHD1 heterozygous mice had a total decrease in fat mass, but in comparison to the total body weight it did not show a significant difference to wild type mice (Figure 12B).

The IMPC data clearly linked CHD1 to the maintenance of normal bones in mice. However, bone formation is a complex process involving different cell types such as MSC, osteoblasts, osteoclasts or osteocytes. Thus we wanted to further specify if MSC differentiation was particularly involved in the defective bone formation with low levels of CHD1. 
A

Chd 1 - Bone Mineral Density (excluding skull) $\left(\mathrm{g} / \mathrm{cm}^{2}\right)$

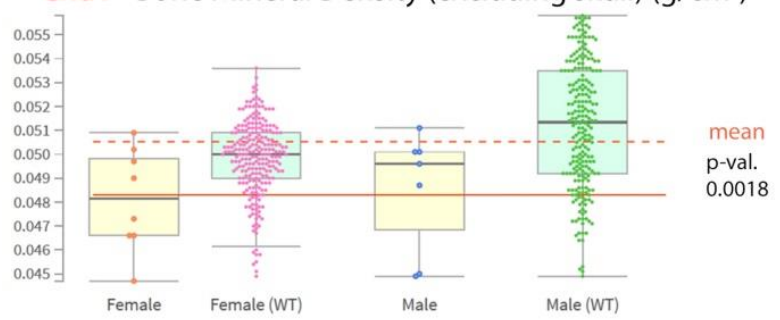

Chd 1 - Body length $(\mathrm{cm})$

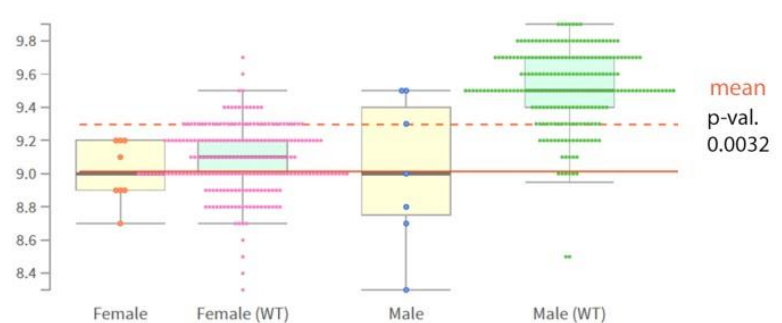

Chd1 - Bone Mineral Content (excluding skull) (g)

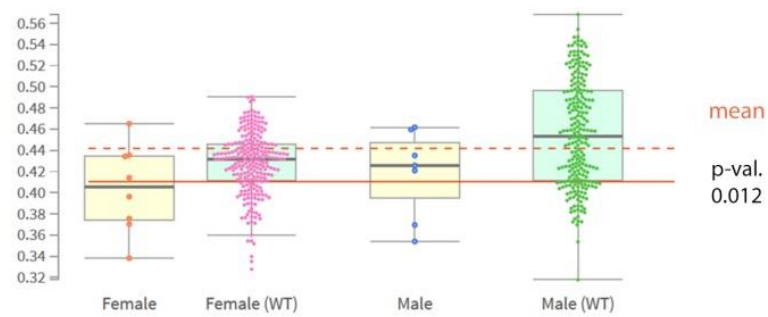

B

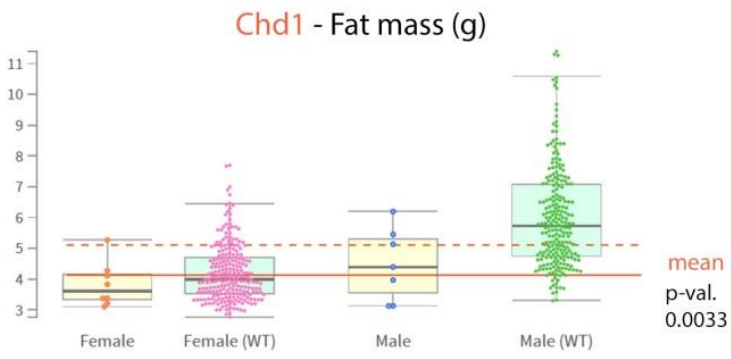

Chd 1 - Fat/ Body weight (ratio)

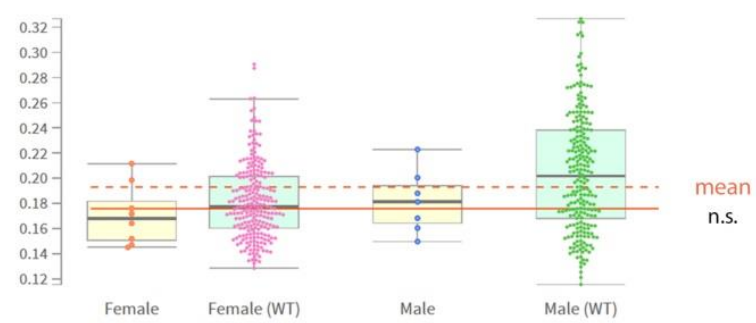

Female $\square$ Female (WT) O Male $\square$ Male (WT)

$\square$ Mutant $\square$ Wild type $\quad---$ Wild type

Figure 12: CHD1 heterozygous knockout mice show decreased bone associated parameters. Box plots represent different phenotypical parameters measured in CHD1 heterozygous C57BL/6NCrl $\mathrm{CHD} 1^{\mathrm{tm} 1 \mathrm{~b}(\mathrm{KOMB}) \mathrm{Wtsi}}$ knockout or wildtype mice described by the international mouse phenotype consortium (IMPC). A Female or male mice were analyzed for bone mineral density, body length, bone mineral content or B fat mass and fat / body weight ratio 14 weeks after birth. Statistic differences between the means of heterozygous CHD1 knockout (female and male; orange line) and wildtype (female and male; dotted orange line) mice for the different parameters were compared by Fisher's exact test. 


\subsection{CHD1 depleted MSC form less ectopic bone in mice}

As described by others MSC formed ectopic bone in vivo after implantation into severe combined immunodeficiency (SCID) mouse (Simonsen et al., 2002). Ectopic bone formation is an easy and meaningful experiment to predict the bone formation capacity of a model system (Abdallah et al., 2008). To test if CHD1 depletion impaired bone formation in vivo we created MSC cell lines which stably express a shRNA-targeting CHD1. In total, three cell lines, two with individual shRNA against CHD1 (shCHD1-\#1, shCHD1-\#2) and one control cell line, expressing a nontargeting control shRNA (shCon), were established and analyzed for CHD1 depletion (Figure 13C). The cells were injected together with a hydroxyapatite/ tri-calcium phosphate (HA/TCP) matrix into SCID mice, which were sacrificed 8 weeks after injection. The ectopically formed tissue sections were stained by hematoxylin and eosin (H\&E) and the ratio of formed bone area (BA) relative to the measured tissue area (TA) was calculated. To control whether that the formed bone tissue was of human MSC origin control sections were immunostained for human vimentin (VIM).

Besides the stable shRNA-expressing MSC untransformed MSC were also injected into mice to exclude effects due to the viral integration into the genome. Untransformed MSC and shCon MSC showed no difference in their mean bone formation rate with $3.2 \%$ and $3.1 \% \mathrm{BA} / \mathrm{TA}$ and were combined into one control group (control). Strikingly, both CHD1 depleted MSC cell lines shCHD1 \#1 and shCHD1 \#2 formed less extracellular collagen matrix compared to control with $0.54 \%$ and $1.12 \%$ BA/ TA, respectively (Figure 13A, B). Statistical analysis by Tukey multiple comparisons with a $p$-value threshold of 0.05 showed a significant difference between the control group and shCHD1 \#1 and shCHD1 \#2, but no difference between shCHD1 \#1 and \#2 (Figure 13B). These findings support the RNA-seq results, highlighting a defect in MSC early osteoblast differentiation with decreased protein levels of CHD1. Furthermore, the low formation of extracellular collagen matrix implies differentiation defects before day five as others showed that deposition of organic compounds began around the fifth day of osteogenesis (Kulterer et al. 2007). 
A
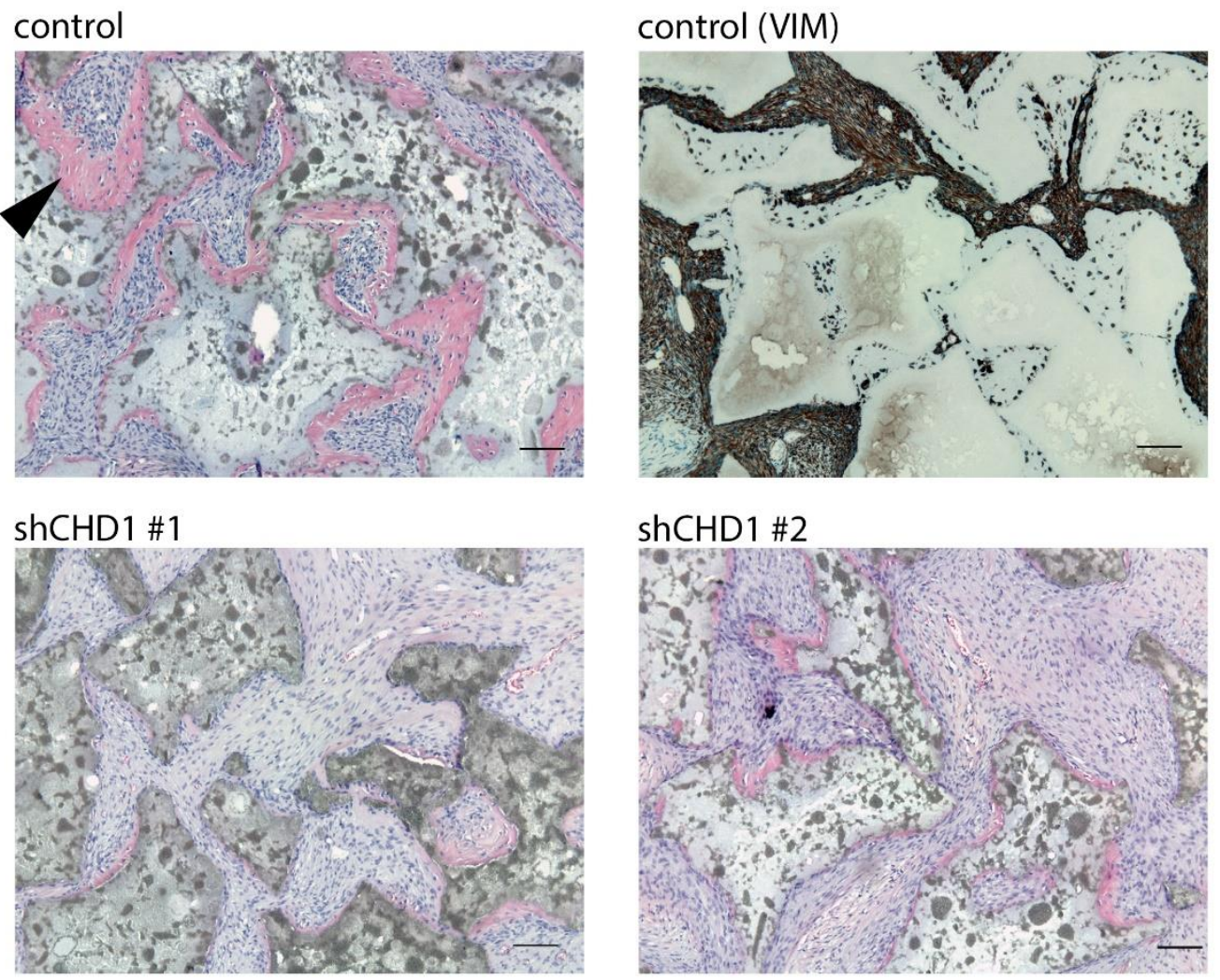

B

C
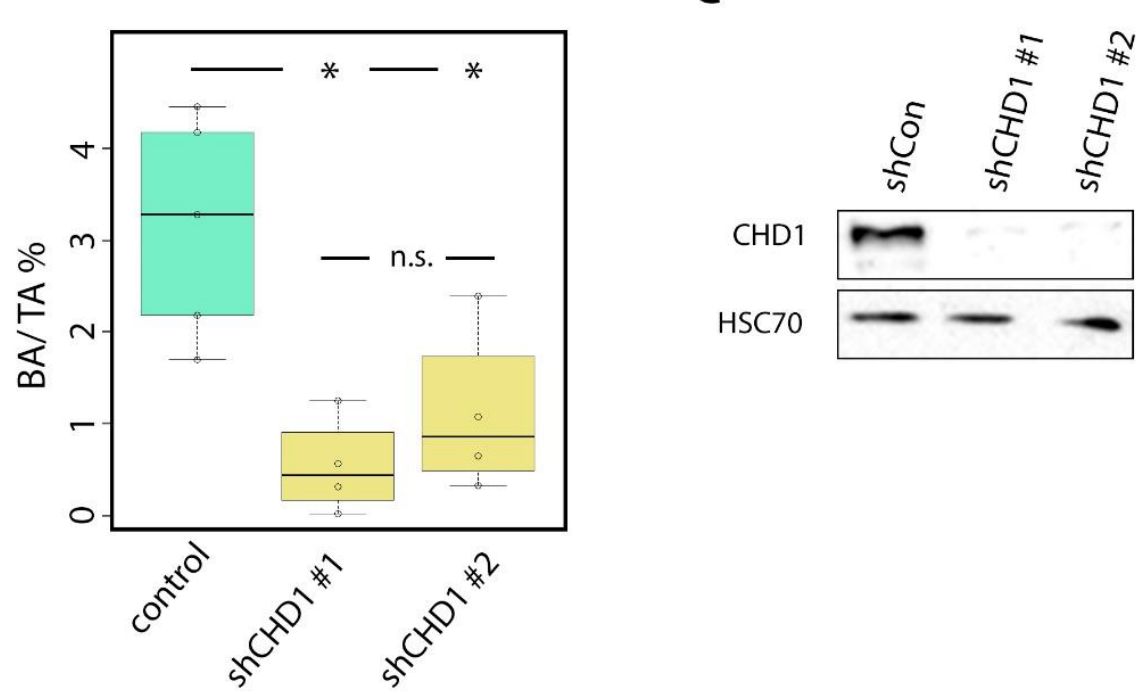

Figure 13: CHD1 depletion reduces ectopic bone formation in mice. A H\&E stained tissue slides show ectopic formed bone area (black arrowhead) of control MSC (non-targeting shRNA) or shRNACHD1 expressing MSC (shCHD1 \#1 or \#2) in severe combined immunodeficiency (SCID) mice. MSC cell lines were subcutaneously injected into mice in a hydroxyapatite/ tri-calcium phosphate matrix. Eight weeks after injection mice were sacrificed and tissue sections were analyzed for BA relative to tissue area (TA) measured. Control slides were immunostained for human vimentin as a control for MSC formed tissue (control (VIM)). The scalebar $=100 \mu \mathrm{m}$. B Boxplot analysis show the ratio of bone area formed relative to tissue area (BA/ TA\%) measured in control MSC $(n=5)$ and CHD1 depleted MSC (shCHD1 \#1, \#2) groups $(n=4)$. Statistical differences between the groups were calculated by Tukey multiple comparison test for a threshold of $0.05\left(^{*}\right)$. Individually measured values are shown by circles. C Immunodetection of CHD1 and HSC70 by Western blot analysis of whole cell protein lysates of stable MSC cell lines used for the ectopic bone formation experiment (see A). 
Taken together CHD1 depletion caused deregulated mRNA expression of differentially expressed genes during adipocyte and osteoblast differentiation in MSC and FOB. The deregulation was characterized by repressed gene induction and attenuated gene silencing (Figure 9). Further, osteoblast with reduced CHD1 levels showed a decreased change of global gene transcription and remained closer to an undifferentiated transcriptomic profile (Figure 8). Moreover, CHD1 depletion increased the correlation to undifferentiated transcription levels in a particular group of genes (Figure 10). A closer analysis on this group of genes showed a significant enrichment in skeletal system and bone development related GO terms (Table 2, Table 3) which was concordant with an abnormal bone phenotype observed in heterozygous CHD1 knockout mouse reported by the IMPC (Figure 12). Finally, CHD1 depleted MSC showed decreased ectopic bone formation in mice (Figure 13). This supported the hypothesis that CHD1 is required for functional osteoblast differentiation by normal regulation of gene transcription and activation of skeletal associated genes. Although this combined was likely caused by effects of CHD1 on the transcriptional processes a direct evidence is missing. Therefore, CHD1 ChIP with subsequent deep sequencing (CHIP-seq) was performed to investigate the mechanism how $\mathrm{CHD} 1$ is regulating these gene transcription changes.

\subsection{CHD1 ChIP-seq reveals high binding near TSS}

Initially the CHD1 ChIP was tested by qPCR (ChIP-qPCR) in undifferentiated conditions in MSC to validate its specificity. The histone modification H3K4me3 ChIP was used as a positive control for TSS-regions and to identify potential CHD1 binding sites. To exclude unspecific signals, a non-specific antibody ( $\lg G$ ) was used as a negative control.

CHD1 ChIP-qPCR signals showed an enrichment downstream of the TSS of the genes ribosomal protein, large, PO (RPLPO), glyceraldehyde-3-phosphate dehydrogenase (GAPDH) and dual specificity phosphatase 1 (DUSP1) (Figure 14A). This specific binding was confirmed by prominent signals of $\mathrm{H} 3 \mathrm{~K} 4 \mathrm{me} 3$, which indicates TSS of either active or bivalent genes. In contrast, CHD1 and H3K4me3 had comparable low signals like IgG around the TSS of the inactive TFF1 gene, which was confirmed by RNA-seq. This together shows specificity of the CHD1 ChIP and made the DNA applicable for genome-wide studies by deep sequencing technique. 
To elucidate global changes of CHD1 binding during differentiation ChIP-seq was performed in undifferentiated, adipocyte and osteoblast differentiated MSC as well as osteoblast differentiated FOB. Additionally, H3K4me3 and H3K27ac ChIP-seq data from MSC of previous studies were used for further analysis. To validate the ChIPseq signal specificity, the obtained sequencing reads of undifferentiated MSC were transformed into genome-wide profiles and compared to the previous ChIP-qPCR enriched regions. Concordantly, both techniques showed an overlapping signal in the tested genome coordinates for CHD1 and H3K4me3 ChIP (Figure 14B). Although the $\mathrm{CHD} 1$ profile had a high overlap with $\mathrm{H} 3 \mathrm{~K} 4 \mathrm{me} 3$, further binding was detected in the gene body and the transcriptional termination site, which suggests independent binding of $\mathrm{H} 3 \mathrm{~K} 4 \mathrm{me} 3$ as well.

A

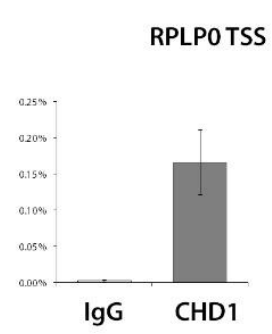

GAPDHTSS

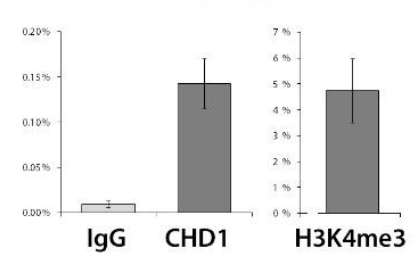

DUSP1 TSS

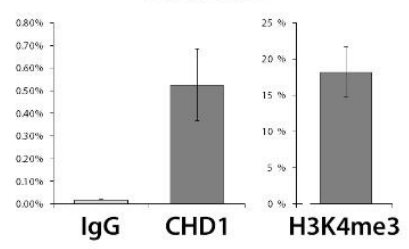

TFF1 TSS

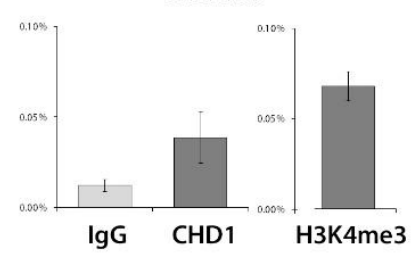

B

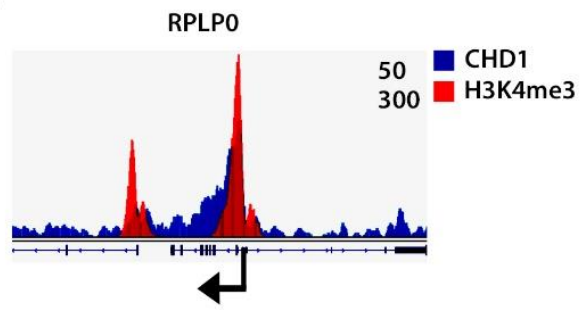

GAPDH

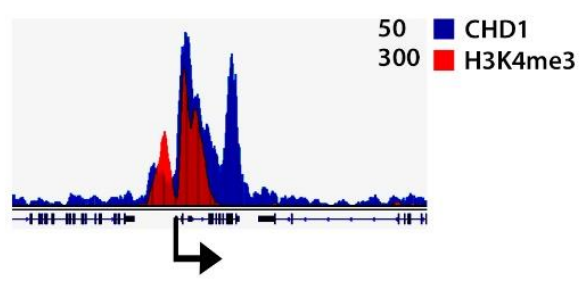

DUSP1

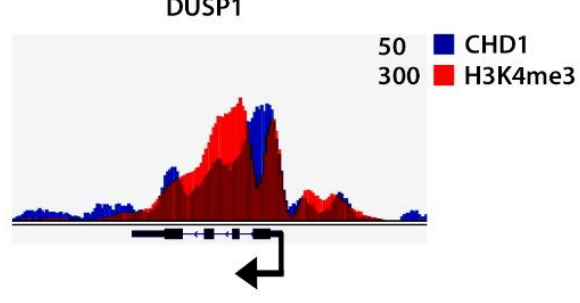

TFF1

$50 \square \mathrm{CHD} 1$

$300=$ H3K4me3

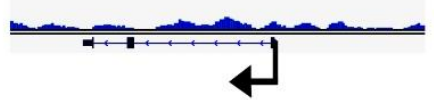

Figure 14: ChIP-qPCR and ChIP-seq profiles of CHD1 and H3K4me3 overlap around TSS. A Bar graphs represent the relative quantification to input of CHD1, H3K4me3 and IgG ChIP signals in MSC at transcriptional start site (TSS) regions measured by qPCR with gene specific primer for ribosomal protein, large, P0 (RPLPO), dual specificity phosphatase 1 (DUSP1), glyceraldehyde-3- 
phosphate dehydrogenase $(G A P D H)$ and trefoil factor 1 (TFF1). Error bars represent standard deviations of biological duplicates. B Genomic profiles show ChIP-seq signals of CHD1 (blue) and H3K4me3 (red) at genes described in A. Signals of CHD1 are shown in a range of $0-50$ of normalized read counts and $\mathrm{H} 3 \mathrm{~K} 4 \mathrm{me} 3$ in a range of $0-300$. The signals were overlaid and visualized by the integrative genomics viewer (IGV). Black arrowheads represent the TSS of respective genes and orientation.

\subsection{Genome-wide CHD1 binding is broadly associated with gene regions}

To elucidate the CHD1 binding pattern and its regulatory role during differentiation, the genome-wide CHD1 binding sites were investigated in undifferentiated, adipogenic and osteogenic conditions in MSC or FOB. All above background and significantly enriched binding sites of CHD1 were overlaid with defined genomic functional segments in the human genome and quantified within them.

The highest proportion of CHD1 binding was observed within the gene regions of introns and exons followed by promoter and $5^{\prime}$ - untranslated regions (5'-UTR) (Figure 15A). However, compared to the high distribution of introns within the genome the relative binding was not strikingly enriched of $\mathrm{CHD1}$. Overall, the binding pattern of CHD1 was quite similar between undifferentiated and differentiated conditions, as well as between MSC and FOB. Interestingly, although CHD1 binding was reported at enhancer regions (Siggens et al., 2015), we could detect only low binding within intergenic distal loci. However, this proportion increased slightly during osteoblast differentiation in MSC. Although mapping of CHD1 to the functional regions revealed its distribution over the genome it did not identify the binding intensities at these sites. Therefore, an average binding profile of $\mathrm{CHD} 1$ to all gene regions was calculated and plotted on a meta gene (Figure 15B). All gene bodies were scaled into a length of 3 kilo base pairs $(\mathrm{kb})$ with an additional region $1 \mathrm{~kb}$ upand downstream of the TSS or the transcriptional termination site (TTS). The highest CHD1 binding was clearly around the TSS and decreased over the gene body. Interestingly, CHD1 signals also increased again at the TTS. This showed that although the most binding regions were associated within the gene body, the highest binding intensities were around the TSS. These observations indicate that the major role of CHD1 is closely around genes with a likely-highlighted function at the TSSregions. 
A

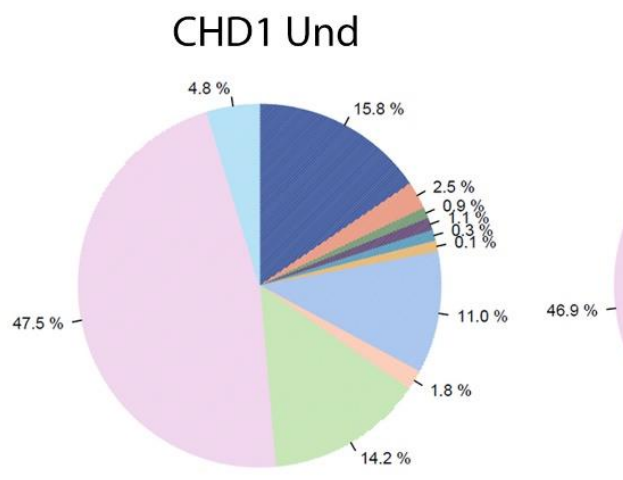

CHD1 Adi

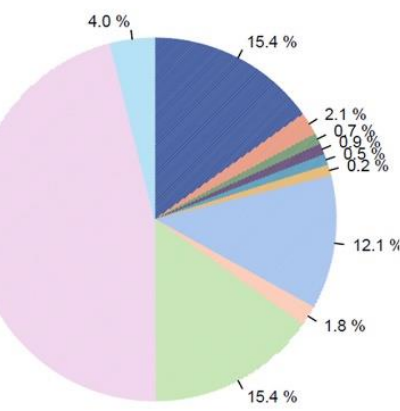

- Promoter (<=1000 bp): $15.4 \%$ Promoter $(1000-2000 \mathrm{bp}): 2.1 \%$ Promoter (2000-3000 bp): $0.7 \%$ Downstream (<=1000 bp): $0.9 \%$

Downstream (1000-2000 bp): $0.5 \%$

Downstream (2000-3000 bp): $0.2 \%$

5'UTR: $12.1 \%$

3'UTR: $1.8 \%$

Coding exon: $15.4 \%$

Intron: $46.9 \%$

Distal intergenic: $4.0 \%$
CHD1 Ost

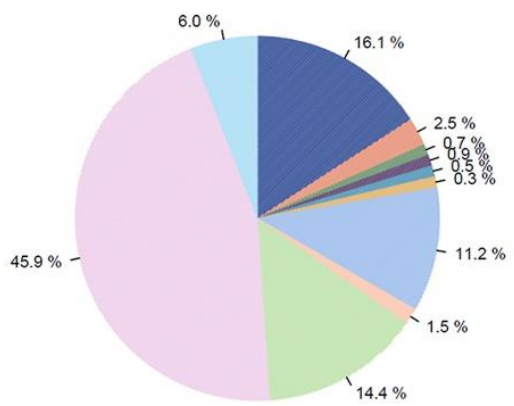

- Promoter (<=1000 bp): $16.1 \%$

- Promoter (1000-2000 bp): $2.5 \%$

- Promoter (2000-3000 bp): $0.7 \%$

- Downstream (<=1000 bp): $0.9 \%$

- Downstream (1000-2000 bp): $0.5 \%$

Downstream (2000-3000 bp): $0.3 \%$

5'UTR: $11.2 \%$

3'UTR: $1.5 \%$

Coding exon: $14.4 \%$

Intron: $45.9 \%$

Distal intergenic: $6.0 \%$
CHD1 FOB

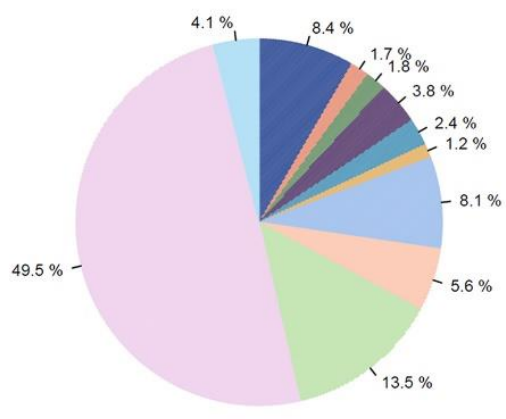

- Promoter (<=1500 bp): $8.4 \%$

- Promoter (1500-3000 bp): $1.7 \%$

- Promoter (3000-5000 bp): $1.8 \%$

Downstream ( $<=1500$ bp): $3.8 \%$

Downstream (1500-3000 bp): $2.4 \%$

Downstream (3000-5000 bp): $1.2 \%$

5'UTR: $8.1 \%$

Coding exon: $13.5 \%$

Intron: $49.5 \%$

Distal intergenic: $4.1 \%$
Genome distribution

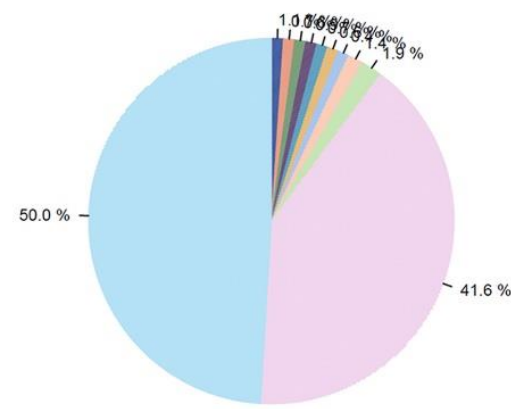

- Promoter (<=1000 bp): $1.1 \%$

= Promoter (1000-2000 bp): $0.7 \%$

- Promoter (2000-3000 bp): $0.6 \%$

- Downstream (<=1000 bp): $0.9 \%$

- Downstream (1000-2000 bp): $0.7 \%$

- Downstream (2000-3000 bp): $0.6 \%$

- 5'UTR: $0.4 \%$

- 5'UTR: $0.4 \%$

Coding exon: $1.9 \%$

Coding exon: 19
Intron: $41.6 \%$

Intron: $41.6 \%$
" Distal intergenic: $50.0 \%$

B

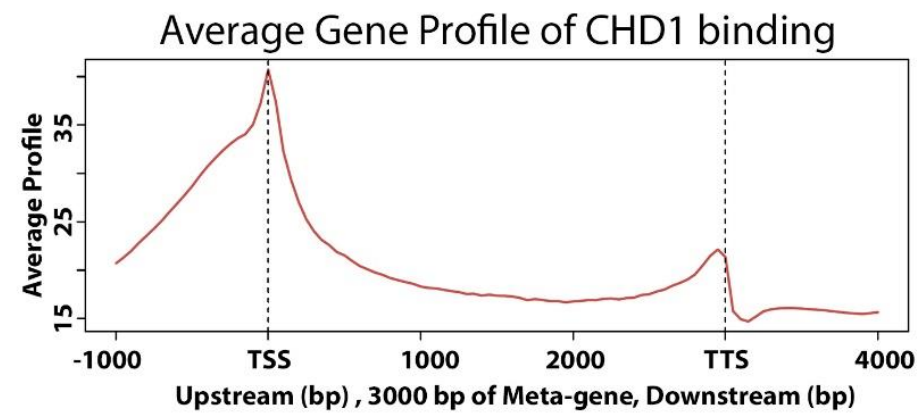

Figure 15: CHD1 distribution on genomic elements. A Pie charts show relative CHD1 binding distribution within functional domains of the human genome in undifferentiated (Und), adipocyte (Adi) and osteoblast (Ost) differentiated MSC or FOB. Significant CHD1 enriched regions were assigned to genomic segments of gene promoter and downstream regions, $3^{\prime}$ - and $5^{\prime}$-untranslated regions (UTR), 
exons and introns and distal intergenic loci. The color table with the respective functional segments and its relative distribution is depicted under the pie charts. The pie chart on the bottom right shows the normal distribution of the functional segments within the human genome (hg19). Calculations and graphics were produced within CEAS software. B Average binding profile represents CHD1 ChIP-seq signals (red line) over all human genes scaled into a meta-gene. The meta-gene includes a region 1,000 base pairs (bp) upstream or downstream of the transcriptional start site (TSS) and transcriptional termination site (TTS) indicated by dotted lines. The averaged CHD1 ChIP-seq signal intensity is represented on the $y$-axis relative to the meta-gene coordinates, shown on the $x$-axis. The representative undifferentiated MSC CHD1 ChIP-seq sample was used to plot the profile by the CEAS software.

\subsection{CHD1 correlates with active histone modifications and gene expression}

As CHD1 is located at and around gene regions and plays a role in gene transcription we sought to further analyze how it was associated with active histone modifications and actively transcribed genes in MSC. H3K4me3 is a hallmark for active and poised TSS whereas H3K27ac mostly decorate actively transcribed TSS. Thus, analysis of binding intensities of both histone marks and CHD1 could clarify which gene states were bound by CHD1 in MSC and in differentiated conditions.

As expected, CHD1 binding correlated well with H3K4me3 binding intensity at TSSregions between 1 and 1,000 bp downstream into the gene body. A Pearson correlation coefficient score between 0.62 and 0.71 was measured in the different conditions (Figure 16A). No obvious differences between undifferentiated and differentiated cell states in the regression lines were observed. Further TSS-regions highly enriched for CHD1 were also decorated with H3K4me3 marks. But surprisingly, high $\mathrm{H} 3 \mathrm{~K} 4 \mathrm{me} 3$ levels were not necessarily highly bound by CHD1. Further, the distribution of $\mathrm{H} 3 \mathrm{~K} 4 \mathrm{me} 3$ intensities was less variable, showing either highly or lowly decorated TSS, but to a lesser extend a fraction in between.

Although H3K4me3 is a descriptive mark for TSS-regions, it does not necessarily indicate active genes. To further investigate the CHD1 binding solely to active transcribed genes the correlation with H3K27ac at TSS-regions was calculated. The observed correlation coefficient in the different conditions was even higher than for H3K4me3 with values between 0.7 and 0.78 (Figure 16A). Additionally, binding levels of CHD1 and H3K27ac showed a tendency for linear correlation, which support the assumption of their co-occupancy at active genes. Moreover, a gradual signal distribution was observed in contrast to H3K4me3 binding patterns around TSS. This could imply a finer readout of gene expression by H3K27ac compared to H3K4me3. Finally, the correlation of CHD1 binding at TSS-regions in MSC and FOB was compared to the respective normalized gene expression of the occupied sites. In all 
conditions the regression line showed a trend between high gene expression and high CHD1 binding intensities (Figure 16B). Notably, also low and medium expressed genes showed medium to high CHD1 binding, especially in MSC osteoblasts. Moreover, the distribution pattern was much broader, supported also by a lower Pearson correlation coefficient between 0.44 in FOB and up to 0.57 in osteoblast differentiated MSC. Summarized, this shows an overall high correlation between CHD1 and active histone marks around TSS. Nevertheless, a lower correlation between CHD1 and gene expression was observed. This was partially caused by low or medium levels of gene expression but relatively high binding of CHD1. 
A
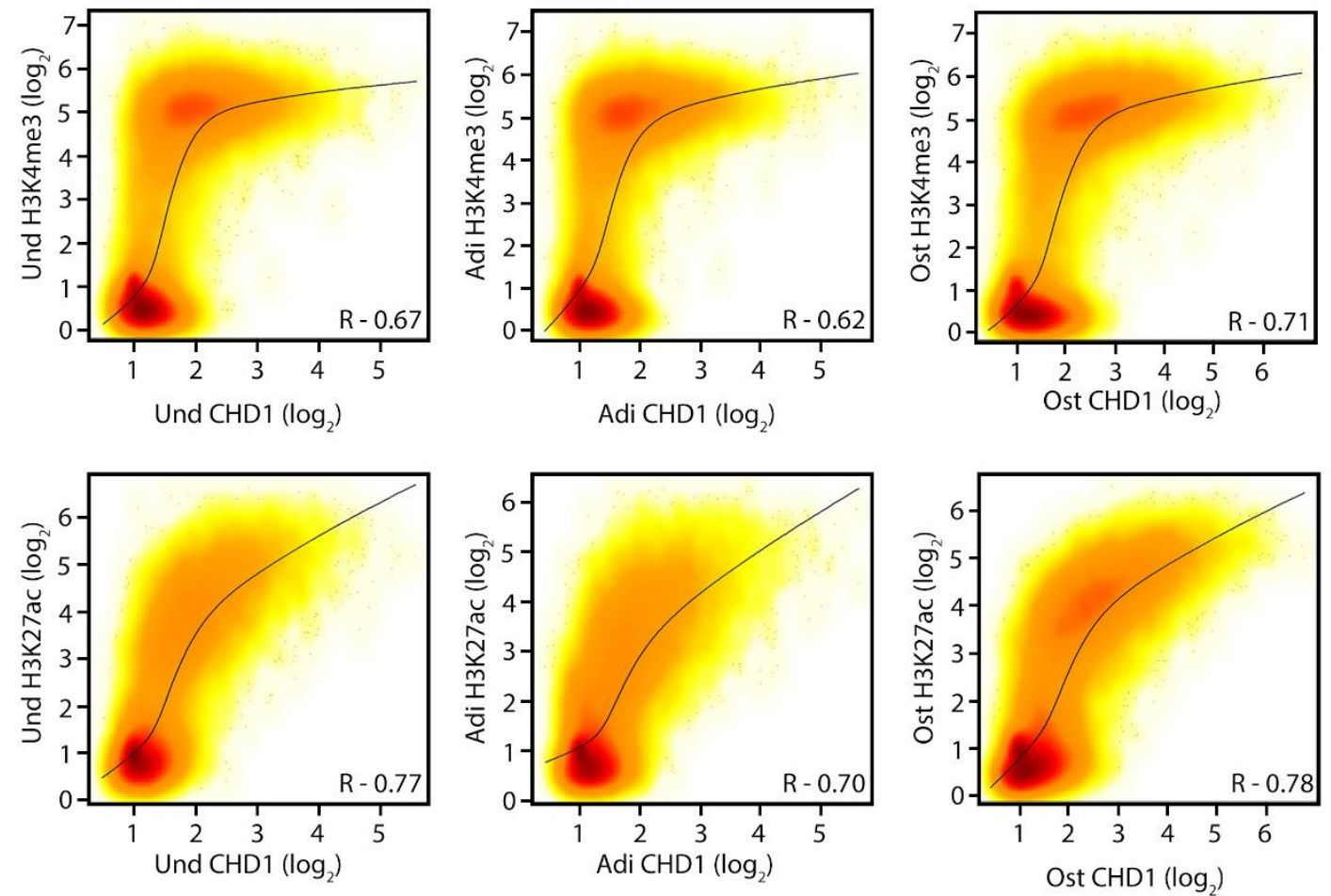

B
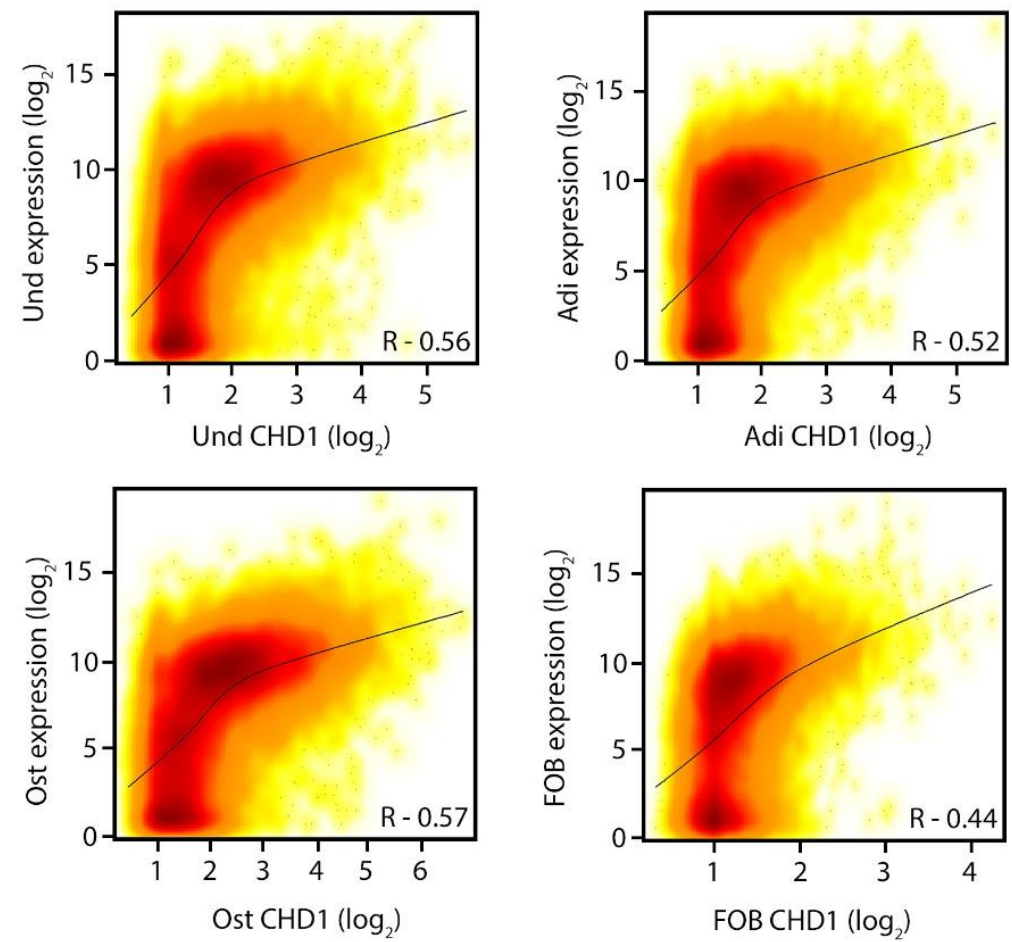

Figure 16: CHD1 positively correlates with active histone marks and gene expression. A Smooth scatter plots show binding intensities of CHD1 (x-axis), H3K4me3 (upper panel) and H3K27ac (lower panel) downstream of the TSS $(0-1 \mathrm{~kb})$. The Pearson correlation coefficient for each dataset is shown at bottom right of the respective plots $(R)$. Black lines represent the regression curves. ChIPseq values of the respective samples in undifferentiated (Und), adipocyte (Adi) and osteoblast (Ost) condition were used for the calculation of binding intensities and were $\log _{2}$ transformed. B Smooth scatter plots depict CHD1 binding intensities (x-axis) against gene expression in undifferentiated 
(Und), adipocyte (Adi) and osteoblast (Ost) condition in MSC or osteoblast differentiated FOB. All human (hg19) genes were used for analysis. For further description see A.

\subsection{Induced genes during differentiation are enriched in CHD1 binding around their TSS}

Since the previous sections showed that CHD1 was variably correlated with gene expression, we especially sought to investigate how differentiation-regulated genes were bound by CHD1. Genes which were induced, unchanged, or repressed during differentiation in MSC were grouped and analyzed for their CHD1 binding at their TSS-region. Subsets were formed with thresholds as described before (Figure $9,+$ $0.5 \log _{2}$ FC, p-adj. < 0.05).

Adipocyte- and osteoblast-differentiated genes which were induced during differentiation had increased CHD1 binding downstream of their TSS, compared to unregulated or repressed genes (Figure 17A, B). Additionally, when comparing CHD1 binding in the undifferentiated state to these genes no or minor differences in their average profile were observed. These findings strongly suggest that genes which were induced during differentiation required CHD1 for their activation, as many of the activated genes were rather inhibited in their induction after CHD1 depletion (Figure 9A, B).

Next, we investigated if the genes induced during the osteogenesis of MSC had also increased CHD1 binding in FOB. This would imply similarities between the cell lines in osteoblast-specific gene activation and requirement of CHD1 for their induction. Indeed, increased CHD1 binding at TSS-sites of genes activated during MSC osteogenesis were also enriched in FOB differentiated osteoblasts (Figure 17B).

Additionally, it was surprising that CHD1 binding signals were higher in osteoblastdifferentiated MSC than in undifferentiated or adipocytes. The reason is unknown, but it can be hypothesized if this was due to higher binding affinity, more recruitment by cofactors or technique procedures. However, binding patterns, gene expression levels and protein levels of CHD1 were not altered in osteoblast or undifferentiated condition (Figure 6B, C, Figure 15A).

A key question was if CHD1 binding around TSS was directly connected to CHD1 mediated gene regulation. To answer this question, all TSS-regions were subset into genes which were higher, lower, or unregulated after CHD1 depletion. Genes were therefore classified by thresholds described before (Figure $9,+/-0.5 \log _{2} \mathrm{FC}, \mathrm{p}$-adj. < $0.05)$ and their mean CHD1 binding was calculated in the region between the TSS 
and $1 \mathrm{~kb}$ downstream. This analysis showed that genes which were less expressed after CHD1 depletion had significant higher CHD1 binding compared to unregulated or higher expressed genes (Figure 17C). Strikingly, this observation was consistent between all differentiation conditions and in MSC as well as FOB. Higher expressed or unregulated genes were not significant differential bound or had low differences in binding. This key finding associated CHD1 protein occupancy around the TSS with direct transcriptional regulation of these respective genes.

Previously, we showed that CHD1 binding was increased at TSS-regions of genes induced during MSC differentiation. Furthermore, these genes were likely to be repressed after CHD1 depletion. Thus, we asked if the genes higher expressed during differentiation, but repressed with CHD1 depletion, were further enhanced in their CHD1 binding around TSS. Indeed, a significant increased CHD1 binding was measured at these TSS-regions (Figure 17D). In FOB however no significant difference was detected (not shown). These findings further support the idea that CHD1 is required for full induction especially of differentiation-activated genes. 
A
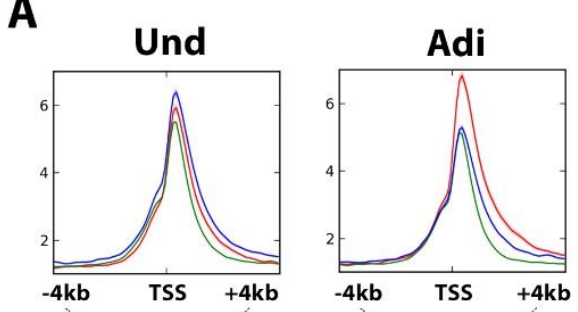

$\Delta$ ร
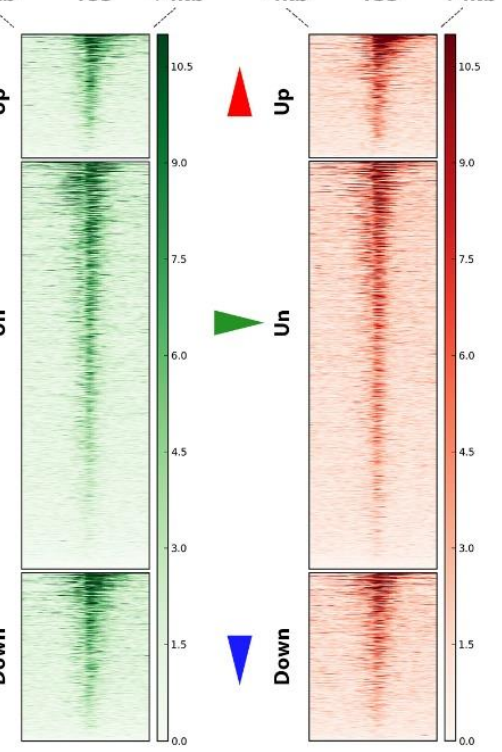

C
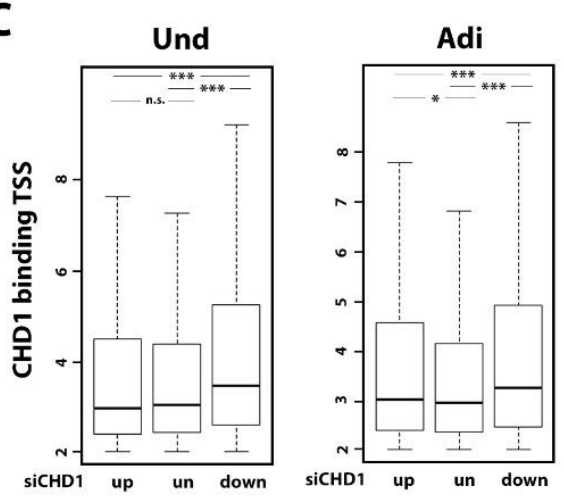

B

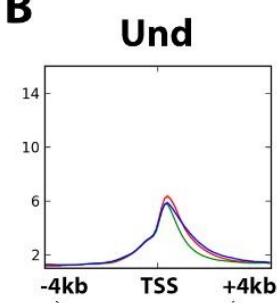

$\Delta$ ร

5

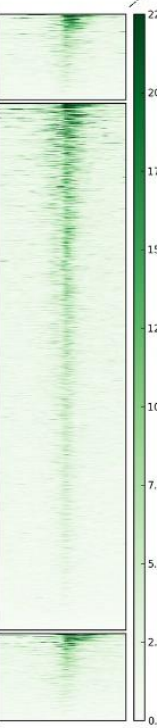

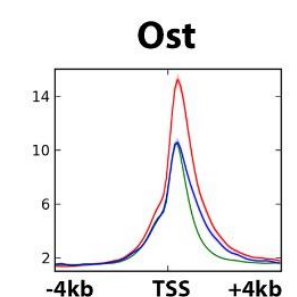

$\Lambda$

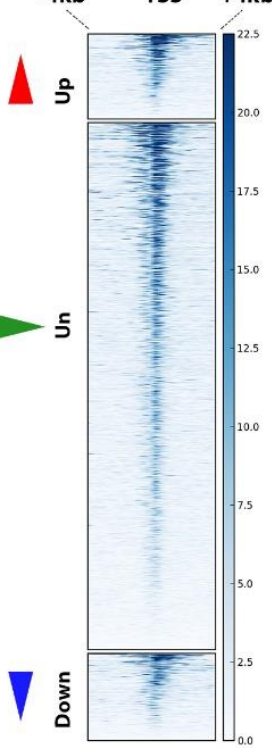

$-5$

$\boldsymbol{\nabla}$

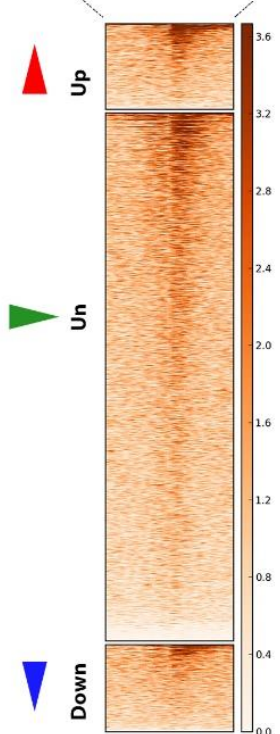

D diff. Adi $\Lambda_{\text {Up }}$
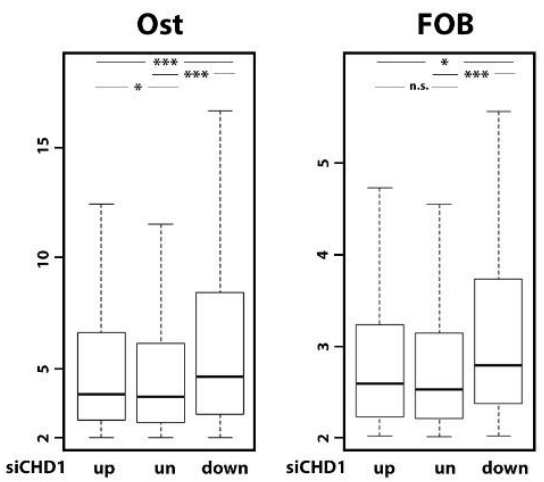

gene expression + siCHD1

$\nabla$ Down

Unchanged

$\triangle$ Up

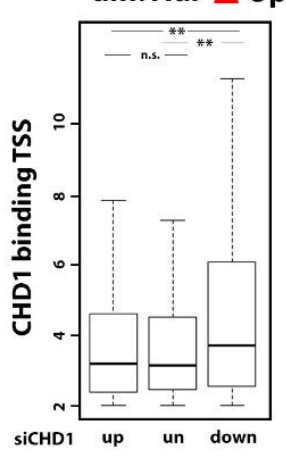

diff. Ost $\Lambda$ Up

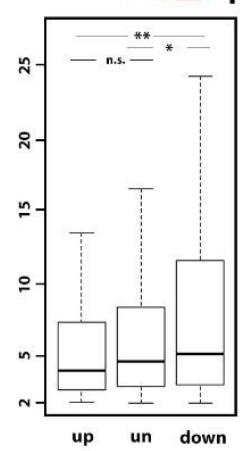

Figure 17: CHD1 binding is enriched near the TSS-regions of induced genes during MSC differentiation. A Heat maps represent CHD1 ChIP-seq binding signals in undifferentiated (Und) or adipocyte differentiated MSC (Adi) relative to a $4 \mathrm{~kb}$ region around the centered TSS of genes which were activated (Up, red arrowhead), unchanged (Un, green arrowhead) or repressed (Down, blue 
arrowhead) after five days of adipocyte differentiation. Genes were selected based on RNA-seq analysis with previous described thresholds (Figure $9 \mathrm{~A},+/-0.5 \log _{2} \mathrm{FC}, \mathrm{p}$-adj. < 0.05 ). The color keys are shown at the right side of the respective heat map. Aggregate plots above the heat maps represent the mean CHD1 binding values with their standard deviation for the respective regulated gene set indicated by the color code described before. B Heat maps show CHD1 ChIP-seq binding in undifferentiated (Und), osteoblast differentiated MSC (Ost) or osteoblast differentiated FOB (FOB) to regions described in $A$ of genes regulated during MSC osteoblast differentiation with thresholds described before (Figure 9B, $+/-0.5 \log _{2} \mathrm{FC}, \mathrm{p}$-adj. <0.05). For further figure explanation see A. C Box plots represent mean CHD1 binding values near TSS of genes regulated by CHD1 depletion in undifferentiated, adipocyte or osteoblast differentiated MSC or osteoblast differentiated FOB. Gene regions between the TSS and $1 \mathrm{~kb}$ downstream of higher (up), lower (down) or unregulated (un) expressed genes after siCHD1 transfection were used to calculate normalized CHD1 ChIP-seq values to. Unbound or below background detected TSS were subtracted from calculation. Statistical analysis was performed by Wilcoxon-rank sum test (n.s. - non significant, ${ }^{*} p$-val. $<0.5,{ }^{* *} p$-val. $<0.01,{ }^{* * *} p$ val. < 0.001). D Box plots depict CHD1 binding values to regions as described in $\mathrm{C}$ of genes activated during adipocyte or osteoblast differentiation in MSC. Further these genes were grouped by CHD1 regulation. For further description see $\mathrm{C}$.

\subsection{Single gene analysis reflects genome-wide observed effects}

Next, we sought to test whether the genome-wide findings of CHD1-dependent regulation on genes by binding to their TSS-regions could also be observed on the single gene level. Therefore, ChIP-seq profiles of CHD1, H3K4me3, H3K27ac, H3K27me3 in osteoblast differentiated and undifferentiated conditions together with RNA-seq signals were compared. Genes, which were transcriptionally induced during osteoblast differentiation and either repressed (SIX1, DUSP1, DUSP5) or enhanced (ALPL, BGLAP) after CHD1 depletion were analyzed. Epithelial membrane protein 3 (EMP3) was selected as an unregulated control gene.

As expected, these selected genes, except for the negative control, showed as expected an increase of the activating marks H3K4me3 and H2K27ac (Figure 18), whereas the repressive mark H3K27me3 was barely detectable except for ALPL. This indicates that these genes were probably not primed for transcription-activation before differentiation. Interestingly, ALPL showed only minor changes in H3K4me3 and H3K27ac, but H3K27me3, a repressive mark, was high in undifferentiated and still weakly present in differentiated state. Most intriguingly, however, was the strong increase of CHD1 binding at the TSS-regions of the activated genes SIX1, DUSP1 and DUSP5 after differentiation. Nevertheless, only a minor increase of CHD1 binding was observed around the TSS of ALPL and BGLAP which were upregulated after CHD1 depletion. In sum, these findings support the role of CHD1 specific regulation at the TSS of differentiation-induced genes in osteoblasts. Additionally, it 
suggests that the marker genes $A L P L$ and $B G L A P$ were probably indirectly regulated after CHD1 depletion.

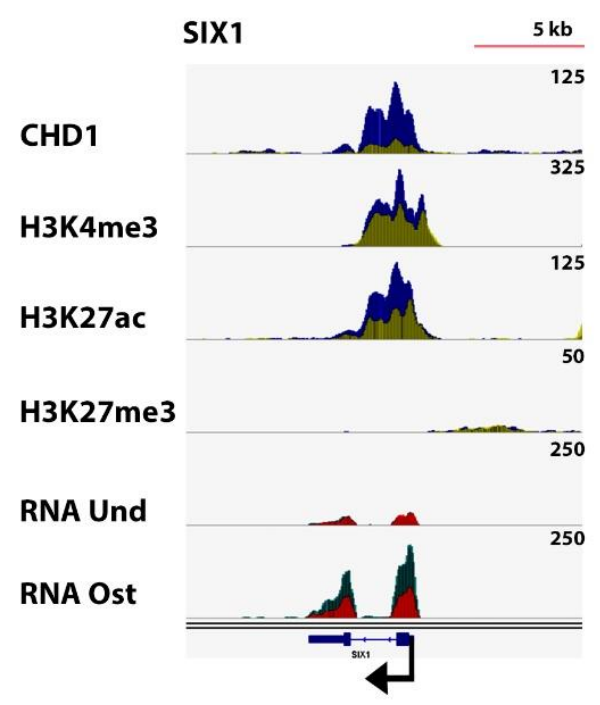

Und

ALPL

CHD1

H3K4me3

H3K27ac

H3K27me3

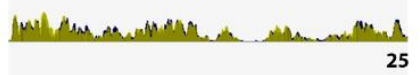

RNA Und

RNA Ost.

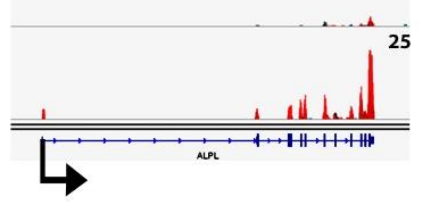

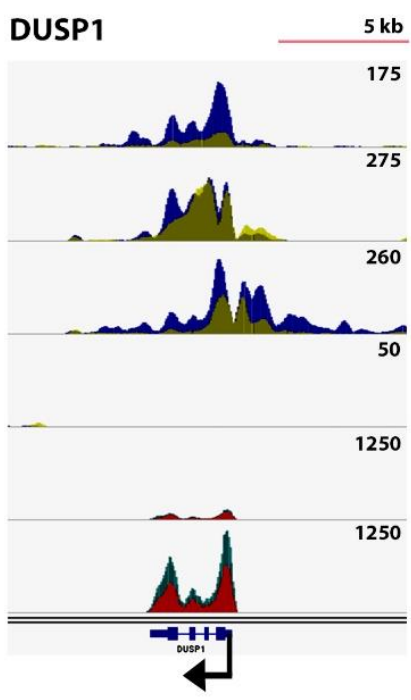

Ost

BGLAP

siCon
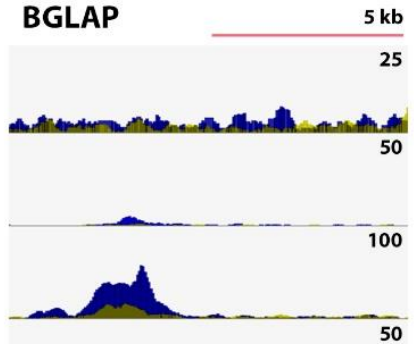

50
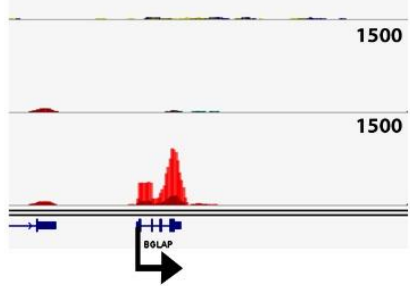

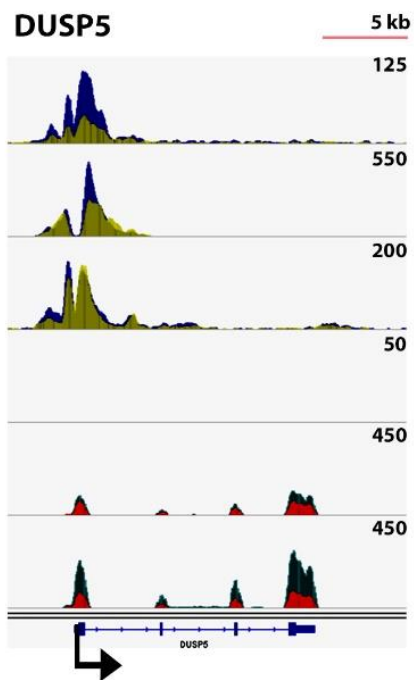

siCHD1

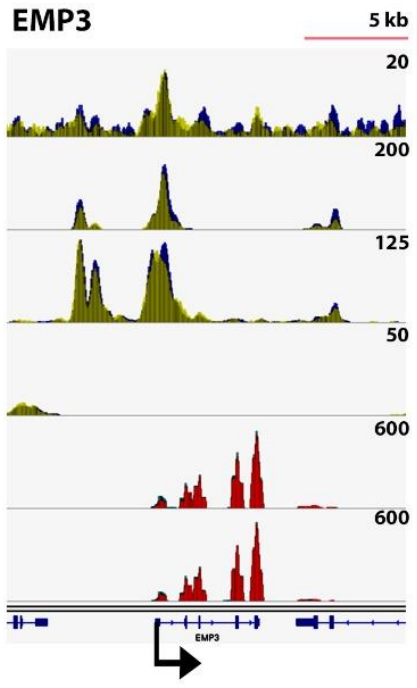

Figure 18: CHD1 binding signals increase around TSS during osteogenesis at CHD1 dependent genes. ChIP-seq profiles of CHD1, H3K4me3, H3K27ac, H3K27me3 in undifferentiated (Und, yellow) and differentiated (Ost, blue) conditions are shown along with RNA-seq signals in siCon (green) or siCHD1 (red) transfected MSC. Genes significantly activated during osteoblast differentiation and repressed by CHD1 depletion (SIX homeobox 1 (SIX1), dual specificity phosphatase 1 (DUSP1), DUSP5, alkaline phosphatase liver/ bone/ kidney ( $A L P L$ ), bone gamma-carboxyglutamate (Gla) protein $(B G L A P)$ ) or unregulated by differentiation and CHD1 depletion (epithelial membrane protein 3 (EMP3)) were selected. Scale bars are shown top right of each panel and represent sizes in kilo base pairs (kb). Normalized read counts are shown top right of each lane and black arrowheads indicate directionality and transcriptional start sites (TSS) of genes. 


\subsection{Global RNA-Pol II occupancy increases around TSS after CHD1 depletion}

In the previous sections we saw that repressed genes after CHD1 depletion had higher binding of CHD1 at the TSS than unregulated or enhanced expressed genes. To address the mechanism of how CHD1 depletion caused less transcription at these genes we decided to analyze the occupancy of RNA-Pol II, H2A.Z and H2Bub1 at these genes in control and CHD1 depleted condition. Previous studies have shown that CHD1 was necessary for RNA-Pol II to overcome the nucleosome barrier downstream of the TSS (Skene et al., 2014). Further, the histone variant H2A.Z, which is also located at the TSS and correlates well with H3K4me3 (Ku et al., 2012) is associated with gene transcription (Zlatanova and Thakar, 2008) and decreased nucleosome stability (Abbott et al., 2001; Henikoff et al., 2009). A connection between CHD1 and H2A.Z had been discussed but was to our knowledge not subjected to an experimental study before (Persson and Ekwall, 2010). As CHD1 is a chromatin remodeler, involved in shifting of nucleosomes and is co-localized with H2A.Z downstream at the TSS we hypothesized that CHD1 depletion could cause alterations in H2A.Z and RNA-Pol II occupancy in FOB. Further, although no global change of H2Bub1 was detected with low CHD1 protein levels gene specific regulation could not be excluded. Therefore, H2Bub1 was analyzed via ChIP-seq to obtain information for specific gene groups and further used as a read-out for gene transcription, as H2Bub1 levels correlate well with transcription elongation levels (Fuchs et al., 2014).

First genome-wide distribution of RNA-Pol II, H2A.Z and H2Bub1 were analyzed in control and CHD1 depleted condition around all TSS. Intriguingly, a genome-wide increase of RNA-Pol II at the TSS was observed when CHD1 was depleted (Figure 19A). Yet, no obvious difference between the total number of bound TSS was observed when comparing the heat maps. As RNA-Pol II binding was recently also described at enhancers we sought to analyze these loci too (Lam et al., 2014). Therefore, FOB specific and active enhancer regions, defined by BRD4, H3K4me1 and H3K27ac occupancy, were analyzed. Interestingly, no difference was observed between the tested conditions (Figure 19B). To exclude that the different binding intensities at TSS were due to higher global RNA-Pol II levels in the CHD1 depleted condition Western blot analysis was performed. We detected no change in the total RNA-Pol II levels by Western blot between control and CHD1-depleted condition (Figure 19C). This in sum supported the idea that higher RNA-Pol II binding at TSS 
sites is independent from total RNA-Pol II levels and restricted around TSS, but not at enhancers. Additionally, CHD1 binding mainly occurred around TSS and less at intergenic regions. This indicates that CHD1 is directly involved in the regulation of these binding occupancies.

The H2A.Z signals showed a biphasic peak around the TSS, flanking the RNA-Pol II peak, as described by others as well (Ku et al., 2012) (Figure 19A). Interestingly, reduced H2A.Z levels downstream and upstream of the TSS were observed when CHD1 was depleted (Figure 19A). Moreover, lower differences in the ratio between the peak height downstream and upstream of the TSS were obvious in CHD1reduced condition. The total number of $\mathrm{H} 2 \mathrm{~A} . \mathrm{Z}$ bound regions however did not change when comparing the signals of the heat maps. Like RNA-Pol II H2A.Z is present at enhancers (Jin et al., 2009). To analyze if the observed effects were also taking place at enhancers, the regions described above were used to map H2A.Z under the different conditions. Surprisingly and contrary to the pattern at the TSS, a slight increase of H2A.Z occupancy at these regions after CHD1 depletion was observed. This suggests that regulation of H2A.Z exchange taking place at the enhancer is different than at the TSS and that latter is likely connected to CHD1. We wondered whether these changed H2A.Z histone distributions at TSS were caused by different H2A.Z protein levels in the cells. Therefore, we performed Western blot to measure the H2A.Z level, but did not detect a change in protein levels of H2A.Z (Figure 19C). Finally, H2Bub1 occupancy to all gene regions between TSS and $5 \mathrm{~kb}$ downstream of TSS were compared in CHD1 depleted and control condition. Concordant with the results of the Western blot (Figure 7A) no global change in H2Bub1 binding was observed at these regions. This also showed that although a global increase of RNAPol II at the TSS was measured, no global increase of the transcription elongation marker H2Bub1 was detected. Further, based on the RNA-seq results less than then percent of all genes were significantly regulated with low levels of CHD1. This indicates that no global effect on gene expression regulation occurred, although increased RNA-Pol II was measured around the TSS. 
A

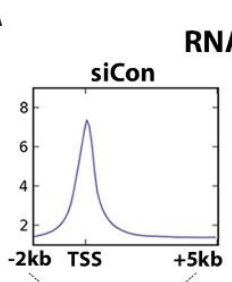

RNA-Pol II
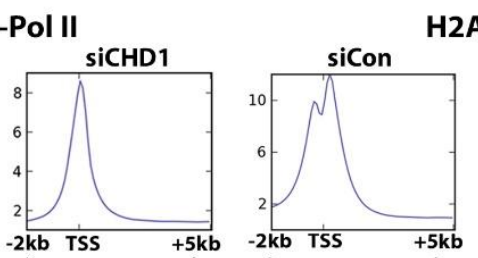

H2A.Z
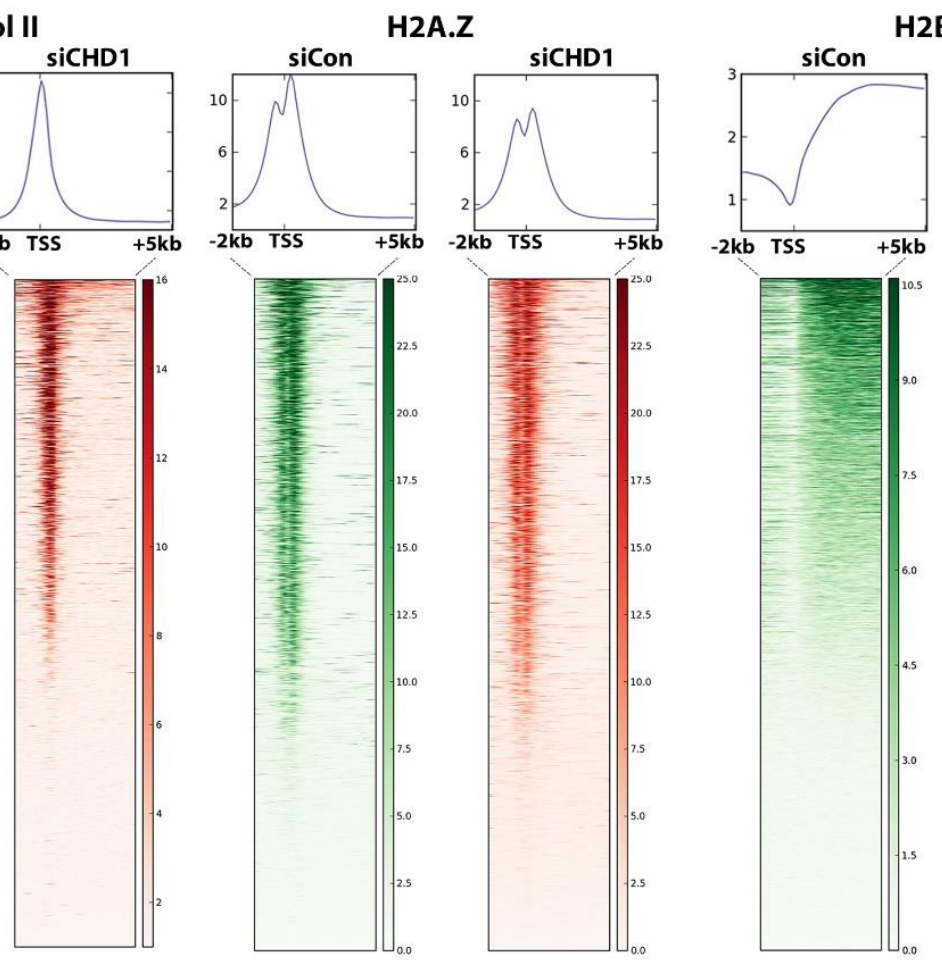

H2Bub1
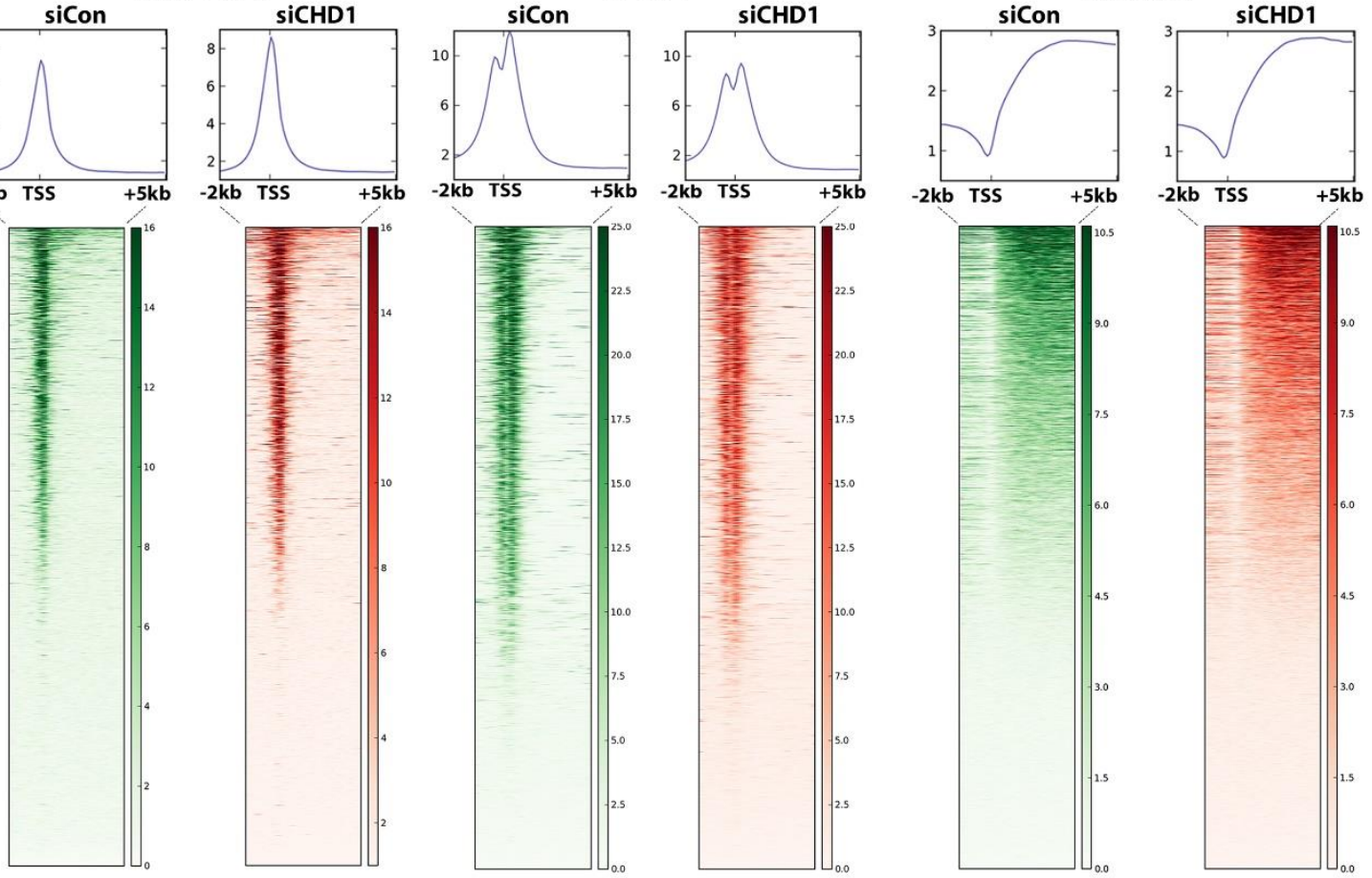

B

Enhancer regions
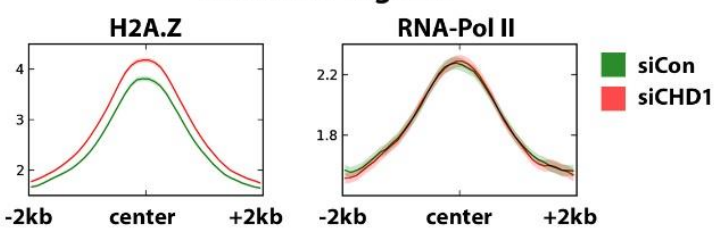

C

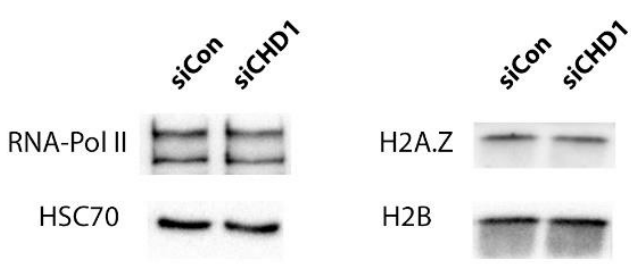

Figure 19: CHD1 depletion increases RNA-Pol II and decreases H2A.Z binding around TSS. A Heat maps represent binding of RNA-Pol II, H2A.Z and H2Bub1 of control (green heat maps) or CHD1-depleted (red heat maps) osteoblast differentiated FOB around TSS. Gene regions were sorted from high to low signal intensity. The average binding profiles above each heat map show the mean binding signal between $2 \mathrm{~kb}$ upstream to $5 \mathrm{~kb}$ downstream of the TSS ( $\mathrm{y}$-axis). The color keys are shown right to the respective heat map. B Average profile plots depict binding of H2A.Z and RNA-Pol II in control (green) or CHD1-depleted (red) condition $2 \mathrm{~kb}$ around active enhancers bound by BRD4. The signal intensities ( $y$-axis) of mean values with standard deviations are plotted relative to the center of enhancer regions. C Immunodetection of RNA-Pol II, HSC70, H2A.Z or H2B with antibodies by Western blot analysis of whole cell protein lysates of differentiated and siCon or siCHD1 transfected FOB. 


\subsection{CHD1 dependent genes show unchanged RNA-Pol II levels around their TSS}

As the global patterns of H2A.Z and RNA-Pol II binding around TSS were impaired after CHD1 depletion, but not all genes were differentially expressed, we sought to further investigate the binding patterns at the CHD1 regulated genes. Surprisingly, RNA-Pol II levels in CHD1-depleted cells were almost unchanged at the TSS-regions compared to control, although the gene expression was reduced after CHD1 depletion (Figure 20A). As expected, enhanced expressed genes showed increased enrichment of RNA-Pol II close to the TSS, but unregulated genes had counter intuitively increased RNA-Pol II signals, as well. However, concordant with reduced gene expression after CHD1 depletion a drop of H2Bub1, a mark for transcriptional elongation, was observed over the gene body (Figure 20A). Moreover, genes with increased transcription after CHD1 depletion indeed showed increased H2Bub1 marks, but, as expected, were transcriptionally unchanged genes not altered in H2Bub1 average levels. This revealed that CHD1-sensitive genes had low transcription levels and indeed reduced H2Bub1 binding over the gene body, but unexpectedly almost unchanged RNA-Pol II occupancy at the TSS. Further, CHD1 unregulated genes also had increased RNA-Pol II levels at the TSS-regions, but neither a change in $\mathrm{H} 2 \mathrm{Bub} 1$ nor in gene expression. This together strongly indicates a stalling of RNA-Pol II when CHD1 protein concentration is reduced.

When comparing H2A.Z levels between the differentially expressed genes, no strong differences were observed in either control or siCHD1 condition, except that enhanced expressed genes had higher levels of H2A.Z in the biphasic peaks (Figure 20A). The almost equal levels of H2A.Z at the TSS in CHD1 reduced condition indicates that the lower H2A.Z occupancy correlated well with genome-wide increased RNA-Pol II at the TSS-regions, however it was not reflecting the change of gene expression caused by CHD1 depletion.

As genes repressed by CHD1 depletion showed lower levels of H2Bub1 and RNAPol II in control condition we were curious to investigate how these genes were expressed before and after differentiation. First, the gene regulation during differentiation of CHD1 regulated genes in FOB was analyzed. Genes which were attenuated in their expression upon reduction of CHD1 protein levels were strong induced during differentiation (Figure 20B). Moreover, genes unregulated or higher 
expressed upon CHD1 depletion showed no or even reduced activation during differentiation, respectively. Interestingly, genes which were sensitive to CHD1 depletion also showed the lowest expression in undifferentiated condition, but were elevated to similar expression levels after differentiation. Most striking however was that the genes in differentiated CHD1-depleted condition were similar in their gene expression levels to undifferentiated status. This is in accordance with previous findings where CHD1 depletion reduced gene expression changes taking place in osteoblast differentiation. Together with the ChIP-seq data, these findings suggest that CHD1 is required for the induction of genes activated during differentiation. It also implicates that the regulation took place downstream of the TSS by affecting early RNA-Pol II-mediated transcription elongation. 
A
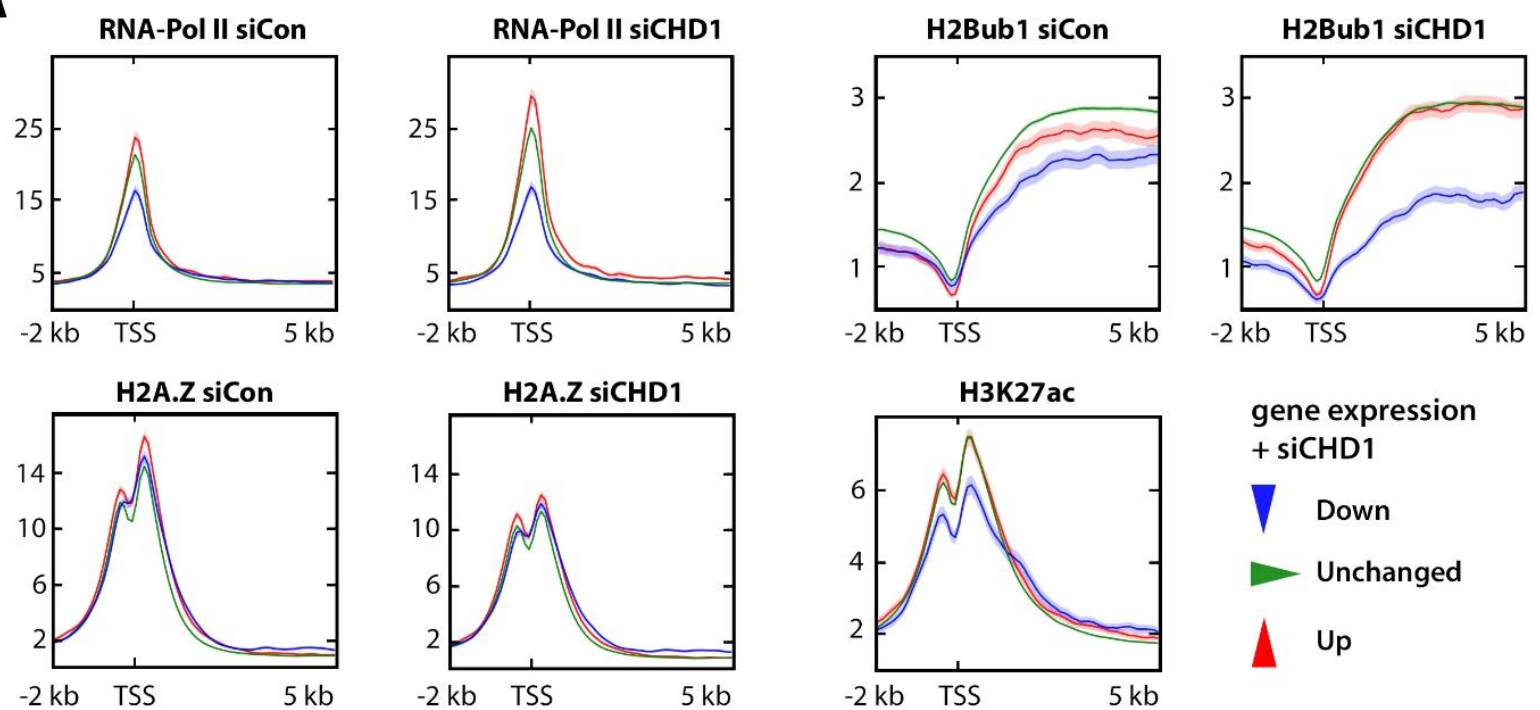

gene expression + siCHD1

$\nabla$ Down

- Unchanged

$\triangle$ Up

\section{B}
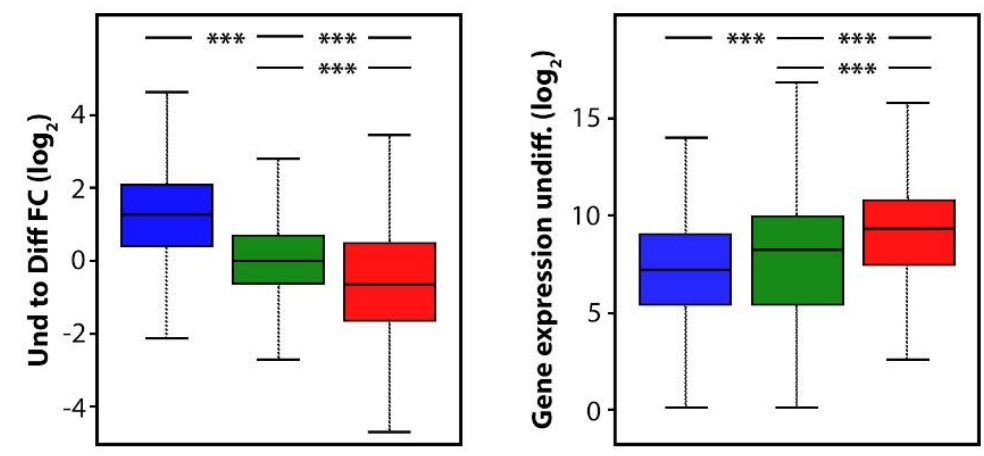

gene expression

+ siCHD1

$\nabla$ Down
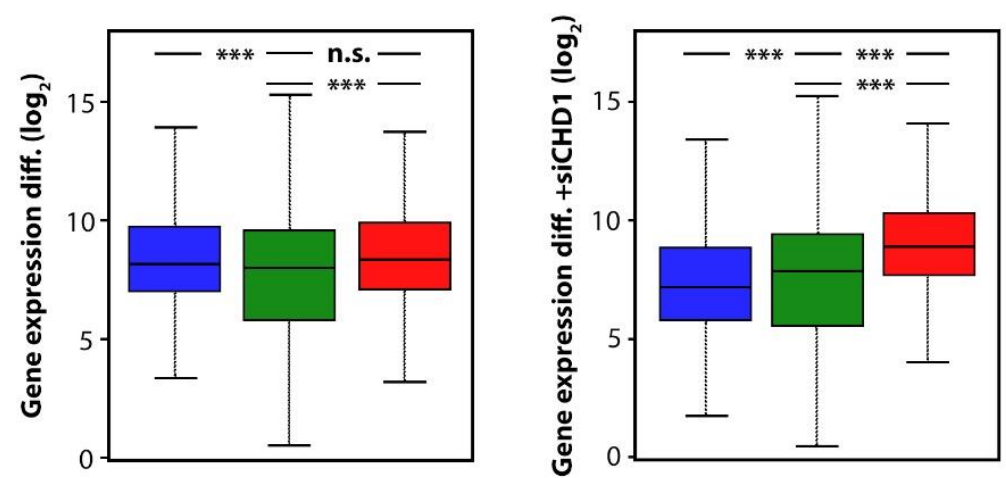

- Unchanged

Up

Figure 20: Unchanged RNA-Pol II occupancy around TSS of repressed genes after CHD1 depletion. A Average profile plots of RNA-Pol II, H2Bub1, H2A.Z binding of control or CHD1 depleted differentiated FOB were plotted relative to the TSS of lower (blue), unchanged (green) or higher (red) expressed genes after CHD1 depletion. Genes were subset by thresholds as described before (+ or $0.5 \log _{2}$ FC, p-adj. $<0.05$, Figure 9A). Mean normalized read counts of respective ChIP-seq samples at $2 \mathrm{~kb}$ upstream and $5 \mathrm{~kb}$ downstream of TSS are shown with their standard deviation. B Box plots depict repressed (blue), unregulated (green) and activated genes (red) after CHD1 depletion for fold changes $(F C)$ in i) gene expression during differentiation (top left box plots), ii) gene expression in undifferentiated FOB (top right box plots), iii) gene expression in differentiated FOB (bottom left box plots) and iv) gene expression in CHD1 depleted differentiated FOB (bottom right box plots). Values 
are shown after $\log _{2}$ transformation. Statistical analysis was performed by Wilcoxon-rank sum test (n.s. - non significant, ${ }^{*}$ p-val. $<0.05,{ }^{* *} p$-val. $<0.01,{ }^{* * *} p$-val. $\left.<0.001\right)$.

\subsection{ChIP-seq profiles reveal RNA-Pol II stalling also on single gene level}

To confirm the observed genome-wide effects on single-gene scale ChIP-seq profiles in FOB of control and CHD1-depleted condition were compared for differently regulated genes. Three representative examples of differentially expressed genes in CHD1-reduced conditions were studied at their genomic region close to the TSS. This analysis of the single-gene profiles also clarified the order of peaks at the TSSregion of CHD1, RNA-Pol II and H2A.Z and their change after CHD1 depletion. First, the CHD1 peak was located downstream of the TSS and the stalled RNA-Pol II (Figure 21). Next, the RNA-Pol II signals were biphasically flanked by H2A.Z peaks, which was well observed for the repressed high mobility group AT-hook 2 (HMGA2), unregulated guanine nucleotide-binding protein $G(I)$ subunit alpha-1 (GNA/1) or higher expressed syndecan 1 (SDC1). Notably, also the H2A.Z profiles at the TSS were decreased when CHD1 was reduced, but peaks upstream of the TSS were less effected, particularly at the higher expressed paired-like homeodomain 1 (PITX1) or the repressed KLF9 gene. This observation of TSS-region independent regulation was concordant with the similar levels of $\mathrm{H} 2 \mathrm{~A} . \mathrm{Z}$ at FOB-specific enhancer (Figure 19B). Further, CHD1 depletion stronger decreased the H2A.Z peak downstream of the TSS, as it was observed at the ELN gene-region.

The ELN gene was of special interest as it is associated with enhanced ectopic bone formation (Larsen et al., 2010; Twine et al., 2014) and repressed by low levels of CHD1 protein (Figure 6C, Figure $7 \mathrm{C}$ ). In this single-gene profile, increased RNA-Pol II levels were detected downstream of the TSS, but decreased levels at the gene body in CHD1-depleted condition. This represents an interesting example of a gene with strong CHD1-dependent regulation. Thus we asked if this pattern of high RNAPol II around the TSS but low-occupancy at the gene body was general for CHD1 
sensitive genes.

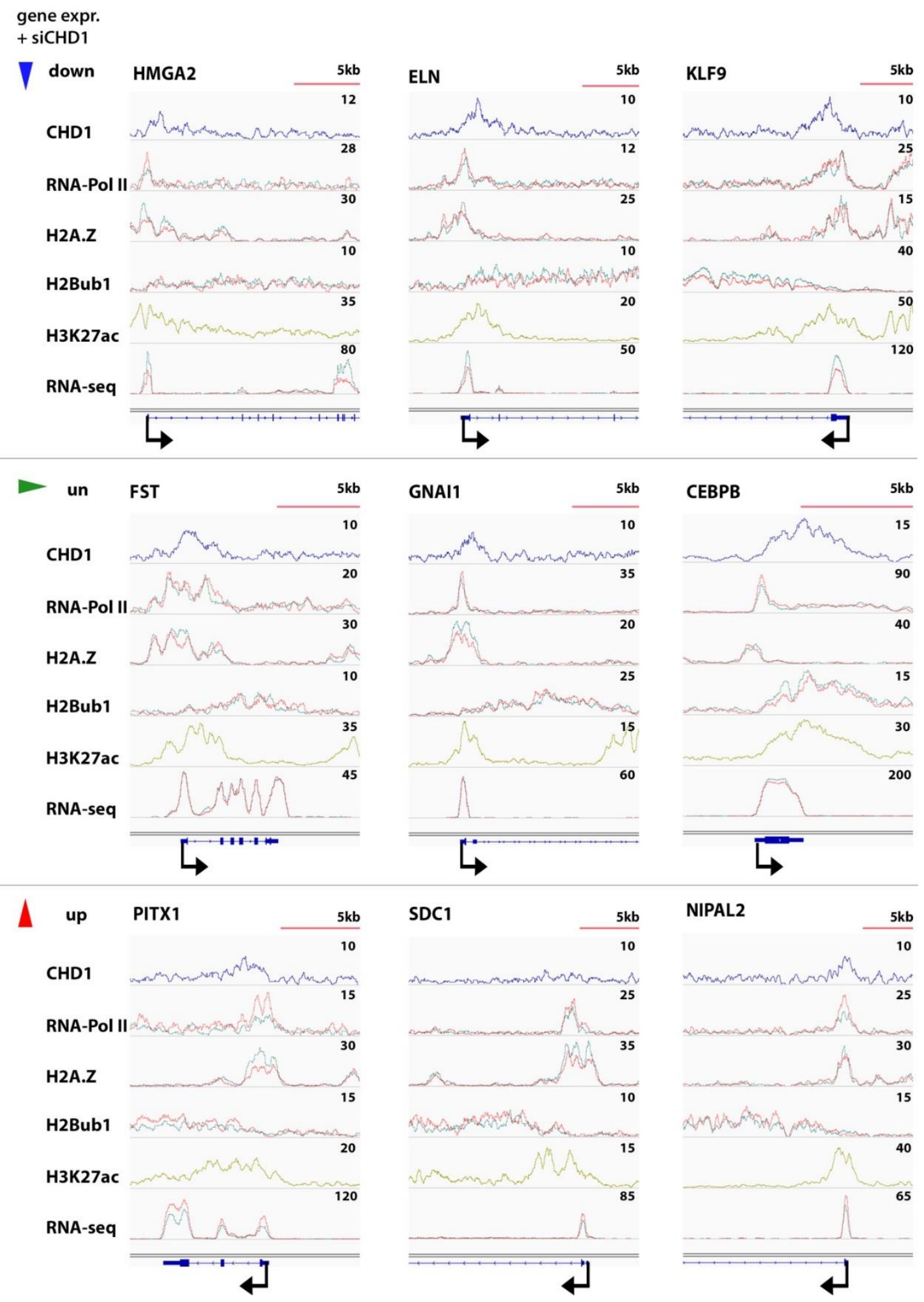

Figure 21: Single-gene profiles describe different pattern of RNA-Pol II profiles after CHD1 depletion. ChIP-seq profiles of CHD1, RNA-Pol II, H2A.Z and H2Bub1 of siCon (green) or siCHD1 (red) transfected FOB are shown along with H3K27ac ChIP-seq and RNA-seq signals. Genes significantly repressed (blue arrow), activated (red arrow) or unchanged (green arrow) after CHD1 depletion were selected as described previously ( + or $-0.5 \log _{2} \mathrm{FC}, \mathrm{p}$-adj. $<0.05$, Figure $9 \mathrm{~A}$ ). Scale bars are shown top right in kilo base pairs $(\mathrm{kb})$ units. Signal intensities are represented in normalized read counts shown top right. Black arrowheads indicate TSS and directionality of genes. 


\subsection{Highest RNA-Pol II stalling ratios at TSS-regions of CHD1 dependent genes}

To further unravel the transcription regulation after CHD1 depletion the genome-wide RNA-Pol II binding was quantified around the TSS and on the gene body (GB) to analyze RNA-Pol II stalling ratios. Therefore, RNA-Pol II intensities were measured in a 300 bp region around the centered TSS and divided by values obtained from the GB (Figure 22A). A similar calculation was described previously by others (Lin et al., 2012). The values were measured for the different groups of genes regulated by CHD1 (+ or - $0.5 \log _{2}$ FC, p-adj. < 0.05, Figure 20A).

First, the binding occupancy of RNA-Pol II around the TSS was compared between the different gene sets in control and CHD1 depleted condition. As observed previously RNA-Pol II values were increased at TSS of unregulated and enhanced transcribed genes, but a decreased median of RNA-Pol II binding at the TSS of repressed genes was measured (Figure 22B). Though, if comparing the mean values, no significant difference was detected (data not shown). Most interesting were the effects observed at the gene bodies. Genes less expressed after CHD1 depletion indeed had significantly less RNA-Pol II binding at the GB. As expected, no difference was observed at unregulated genes. Highly expressed genes had an increased median of RNA-Pol II at the GB, which was, however, not significant. Finally, when comparing the RNA-Pol II ratio between values measured at the TSS and GB, all gene groups showed an increased stalling ratio with low CHD1 protein levels. Intriguingly, we observed the highest difference for repressed genes. This indicates the highest retention of RNA-Pol II is present at genes which are repressed after CHD1 depletion. Although unregulated and highly expressed genes had also an increased stalling of RNA-Pol II around TSS, similar median levels of RNA-Pol II or increased levels of RNA-Pol II at GB were measured in non-regulated or highly expressed genes, respectively. This together indicates that RNA-Pol II is impaired in its early elongation release rate into the gene body in a genome-wide manner and gets stalled at the TSS in CHD1 depleted condition. The fact that still not all genes are reduced in their expression is likely by a compensation of increased RNA-Pol II occupancy at TSS-regions. Genes which are reduced in their mRNA-expression may not have sufficient increase of RNA-Pol II to adapt to the increased stalling ratio. 
A

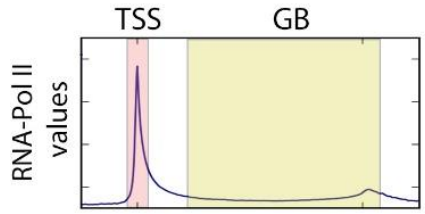

B

RNA-Pol II

RNA-Pol II + siCHD1

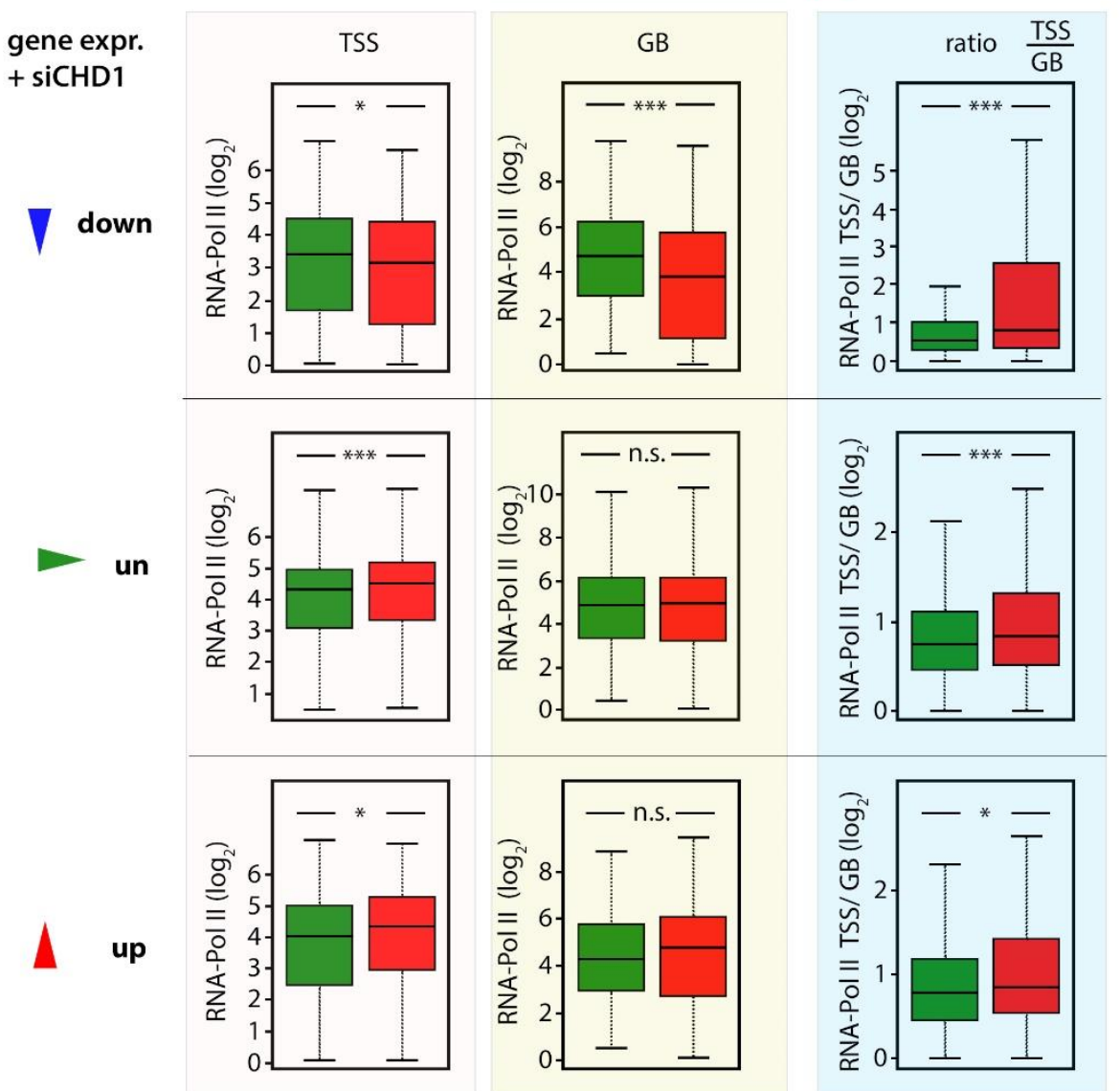

Figure 22: RNA-Pol II stalling ratios are highest in repressed genes after CHD1 depletion. A Schematic average profile plot of RNA-Pol II signals over a meta-gene with the transcriptional start site (TSS) region (red box), defined by 300 bp around the centered TSS and the gene body (GB) region defined as $30 \%$ of gene length downstream of the TSS to $3 \mathrm{~kb}$ downstream of the transcription termination site (TTS) (yellow box). These regions were used to calculate the RNA-Pol II occupancy by the DiffBind software (Lin et al., 2012). B Box plots show values of RNA-Pol II occupancy at the TSS-region, in the GB or the ratio of both values (TSS divided by GB) in control (green) or CHD1 depleted (red) FOB. Measurements of normalized ChIP-seq values were calculated for genes which were less (down), unchanged (un), or higher expressed (up) after CHD1 depletion in FOB (+ or - 0.5 $\log _{2} \mathrm{FC}, \mathrm{p}$-adj. $<0.05$, Figure 20). Statistical analysis was performed by Wilcoxon-rank sum test (n.s. non significant, ${ }^{*} \mathrm{p}$-val. $<0.05,{ }^{* *} \mathrm{p}$-val. $<0.01,{ }^{* * *} \mathrm{p}$-val. $\left.<0.001\right)$. Values depicted were $\log _{2}$ transformed. 


\section{Discussion}

Transcriptional regulation plays an essential role in determining the cell fate during differentiation. Importantly, epigenetic processes are pivotal in coordinating various signals within the cell and respond to the physiological stimuli. Besides histone modifications, the chromatin undergoes extensive remodeling at genes and gene regulatory regions. Though, these regulatory mechanisms are often co-regulatory. The ATP-dependent chromatin remodeler CHD1 is a well described transcriptional co-regulator. However, the mechanistic role of CHD1 during stem cell differentiation is poorly studied. Here, we could show that CHD1 is required for lineage-specific differentiation of MSC and FOB by regulating the expression of differentiation-specific genes. Genome-wide CHD1 occupancy analyses revealed its increased binding at TSS of differentiation-induced genes, thus promoting their transcription activation. Mechanistically we could show that CHD1 depletion caused increased stalling of RNA-Pol II at the TSS region, especially on induced genes during differentiation. Furthermore, global H2A.Z levels around TSS were decreased with CHD1 knockdown, which may cause a higher stability of the +1 nucleosome at the TSS and led to increased stalling. Finally, ectopic bone formation in mice was decreased with constitutive knockdown of CHD1 confirming its role in bone differentiation in vivo. This altogether showed that CHD1 can act as a regulator of stem cell differentiation partly by affecting the stalling of RNA-Pol II and H2A.Z occupancy which regulate differentiation-dependent gene expression. 


\section{CDH1 dependent and induced genes during differentiation}

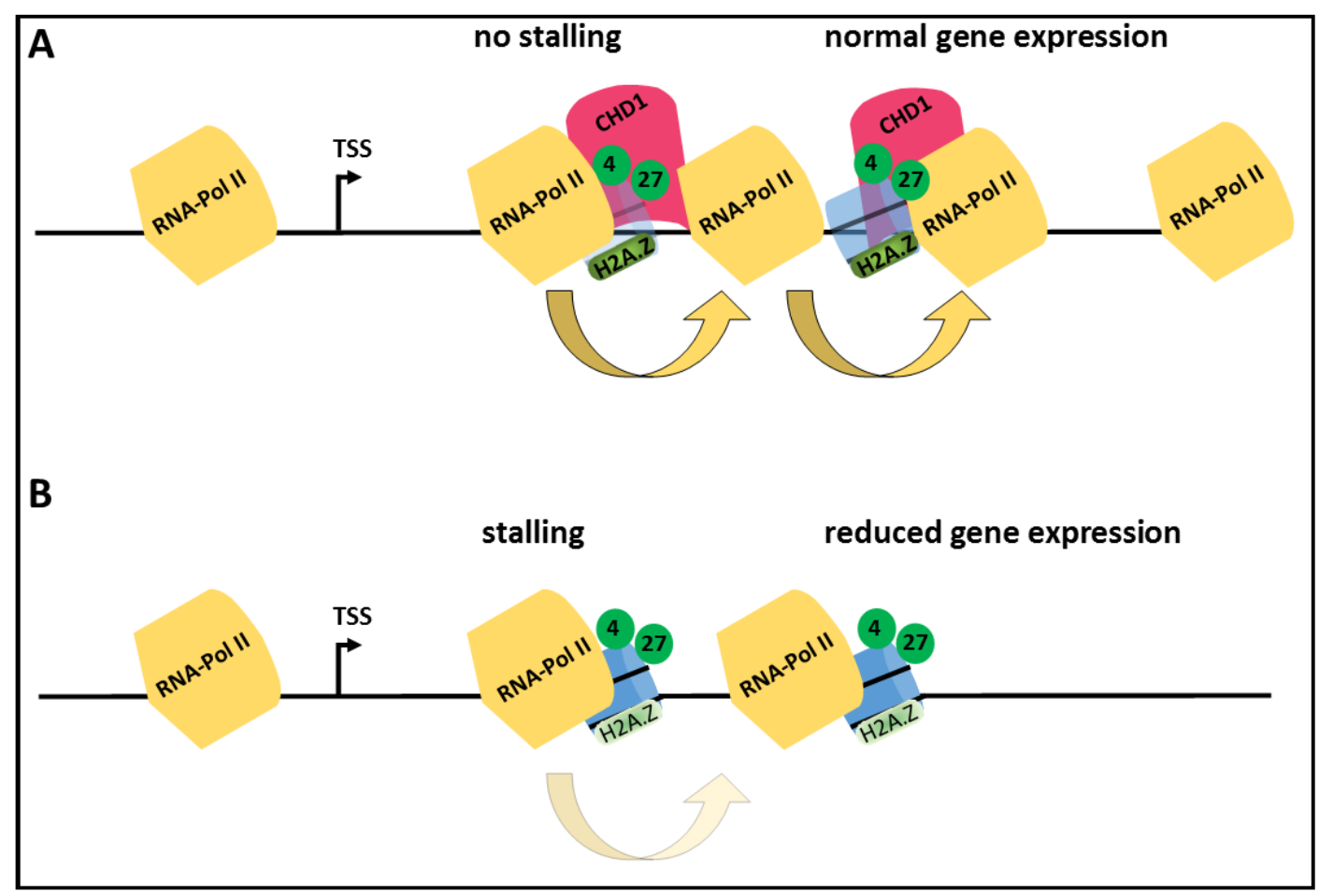

Stable expressed genes

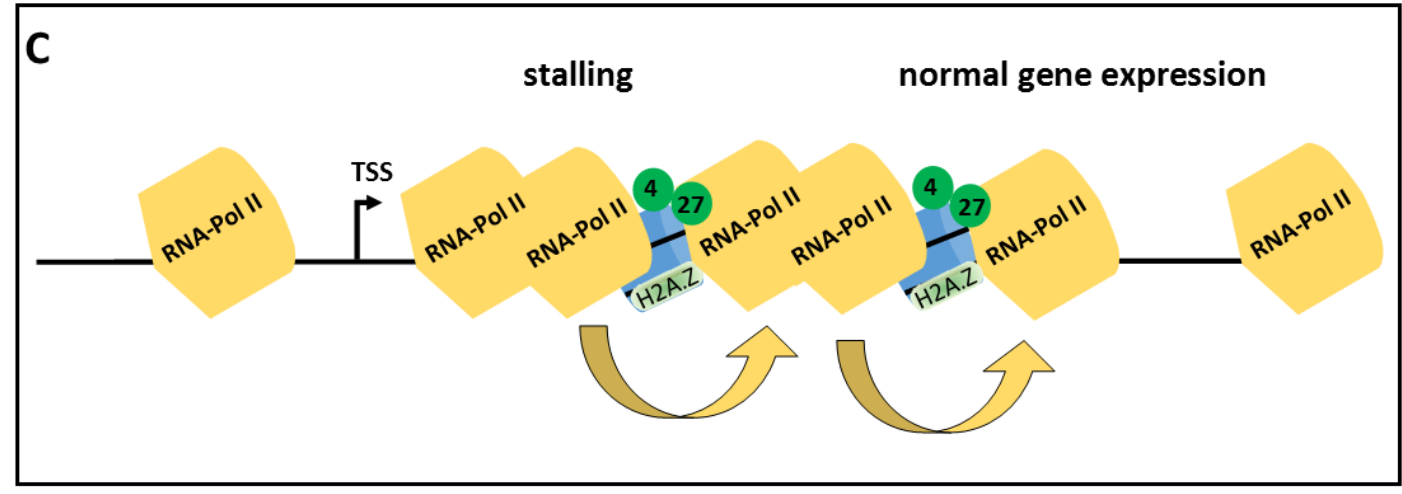

Figure 23 Model of CHD1-regulated gene expression during differentiation of induced and stably transcribed, non-induced genes. A The induced gene is transcriptionally activated and bound by CHD1 (red) which remodels the nucleosome barrier for efficient, early-elongation of the RNA-Polymerase II (RNA-Pol II). CHD1 binding downstream of the transcriptional start site (TSS), close to the nucleosome marked with H3K4me3 (green four) and H3K27ac (green 27), increases the nucleosome turnover and the incorporation of the histone variant H2A.Z. This incorporation further destabilizes the nucleosome for an efficient RNA-Pol II passage. The less intense color of H2A.Z indicates its reduced occupancy. B In the absence of CHD1 the RNA-Pol II is stalled during the earlyelongation process downstream of the TSS. The overcoming of the nucleosome barrier is reduced which causes decreased gene expression for a low expressed and induced gene. Low levels of H2A.Z might further increase the nucleosome stability. C A stable expressed gene which is further induced by differentiation has stalled RNA-Pol II downstream of the TSS as well, when CHD1 is depleted. However increased RNA-Pol II levels around the TSS increase the likelihood of overcoming the nucleosome barrier which leads to unchanged gene expression, even though H2A.Z levels are low. 


\subsection{Genome-wide transcription effects of CHD1 by regulating RNA-Pol II stalling}

CHD1 is described as a global co-activator of gene expression in mice and human (Koh et al., 2015; Sims et al., 2007). However, most of the studies in yeast or mouse observed only mild effects of CHD1 on global gene expression (Gaspar-Maia et al., 2009; Lee et al., 2012a). Nonetheless, only few studies performed genome-wide transcription analyses in a CHD1-impaired system after various stimuli for example by stress or differentiation (Koh et al., 2015; Park et al., 2014). In this study we observed that transcriptional activation was regulated and that CHD1 depletion had differential effects during differentiation. Thus, we hypothesize that CHD1 is required for a cell fate change. Indeed, effects on de-differentiation were observed earlier in mouse when Gaspar-Maia and colleges reprogrammed CHD1-depleted MEFs by introducing the Yamanaka factors, where a significant reduction of iPS colony formation was observed when CHD1 was depleted by RNAi (Gaspar-Maia et al., 2009). A recent in vivo study of the same group showed that CHD1 is necessary for the differentiation of endothelial progenitor cells to HSC by blocking differentiationspecific genes (Koh et al., 2015). However, if genes induced during differentiation or if already stable expressed genes were CHD1-sensitive stayed elusive. With our investigations we were able to show that CHD1 is required for the direct activation of induced genes during osteoblast differentiation. This indicated gene specific regulation. Though, another study reported that CHD1 is necessary for a global increase of transcriptional output in highly proliferating mouse epiblasts, but with decreased RNA-Pol II occupancy levels around TSS in CHD1 knockout embryonic stem cells (Guzman-Ayala et al., 2015). However consistent with our findings, a study in non-embryonic human system observed the RNA-Pol II stalling as the central regulatory mechanism for differential transcription regulation too (Skene et al., 2014).

\subsubsection{How is CHD1 depletion affecting gene expression in particular?}

To elucidate the molecular mechanism of $\mathrm{CHD1}$-specific gene regulation, we studied the occupancy of RNA-Pol II, H2A.Z and H2Bub1 under CHD1-depleted conditions. In consistence with the association of RNA-Pol II with CHD1 in overcoming the +1 nucleosome barrier (Skene et al., 2014), we observed that CHD1 depletion affects global RNA-Pol II stalling. Here we extended the regulatory mechanism by showing that only particular genes were sensitive in differential gene expression to RNA-Pol II stalling after CHD1 depletion. Unaffected and higher expressed genes after CHD1 
depletion gained RNA-Pol II, but repressed genes did not. This led us to hypothesize that the higher nucleosome barrier was overcome by increased RNA-Pol II at the TSS region. Indeed, different in vitro studies have shown that the levels of RNA-Pol II are important to overcome a transcriptional barrier (Epshtein and Nudler, 2003; Saeki and Svejstrup, 2009). Further, it was shown that an increased number of RNA-Pol II closely occupying a DNA region could "collide" and push each other which increased the energy of the first RNA-Pol II to overcome a pause site. Concordantly with this it was observed that a highly expressed DNA template, with more RNA-Pol II bound, were less susceptible for stalling at a pause site than a lower expressed template (Kulish and Struhl, 2001). This indicates why high expressed genes were less affected by CHD1 depletion than the low expressed genes. Summarized these studies show that the increased RNA-Pol II levels at the TSS regions observed by us were sufficient to maintain the normal transcriptional output when CHD1 was depleted. However, repressed genes had similar occupancy levels of RNA-Pol II and were less likely to overcome the nucleosome barrier with low CHD1 protein levels.

Interestingly another study showed in yeast that pausing of RNA-Pol II occurs also over the entire gene body (Churchman and Weissman, 2011). This might extend the role of CHD1 to support the overcome of the nucleosome barrier not only at the TSS, but also at the gene body, as we and others observed CHD1 binding at the entire gene.

Further we saw that particularly genes were highest stalled after CHD1 knockdown which got induced during differentiation. Other studies had observed that genes highly responsive to stimulation or activated during development were stalled before induction and that stalling was reduced after their activation (Muse et al., 2007; Zeitlinger et al., 2007). CHD1 might be important for the activation of these stalled genes. Thus it would be important to test if the genes affected by CHD1 depletion were stalling in undifferentiated condition.

In addition to positive elongation factors, RNA-Pol II release from TSS can be also stimulated by histone modifications like H3K27ac, H3K122Ac or H3K115Ac (Manohar et al., 2009; Stasevich et al., 2014). Surprisingly, in our study, we observed low levels of H3K27ac around TSS of CHD1-sensitive genes irrespective of their expression. Similarly, differentiation-regulated genes which were low in H3K27ac occupancy, but had normal gene expression like stably expressed genes, were described in 
D. melanogaster (Pérez-Lluch et al., 2015). Furthermore, other studies observed the effect of low or no histone modifications in induced and activated genes (Chen et al., 2013; Hödl and Basler, 2012; Zhang et al., 2014). These suggest the dynamic nature of histone code in their regulation of gene expression and the association with CHD1.

\subsubsection{Reduced H2A.Z levels upon CHD1 depletion may link to decreased nucleosome turnover}

Besides higher RNA-Pol II stalling, we observed less occupancy of the histone variant H2A.Z around TSS upon CHD1 knockdown. Interestingly, it was described that H2A.Z incorporation into the +1 nucleosome decreases the nucleosome barrier and that depletion of H2A.Z cause higher RNA-Pol II stalling (Weber et al., 2014). This highly suggests that the lower H2A.Z levels observed after CHD1 depletion caused increased RNA-Pol II stalling. This effect however was not observed at active enhancers and argues for a TSS-specific effect. Yet, if the lower H2A.Z occupancy around the TSS is due a lower steady-state level of the +1 nucleosome or solely due to lower H2A.Z levels can here only be speculated.

What argues for a lower steady-state nucleosome occupancy was the observation that a CHD1 mutant which caused decreased nucleosome turnover at the TSS led to surprisingly lower steady-state levels of the +1 nucleosome (Skene et al., 2014). This would favor a model where the reassembly of the nucleosome after the passage of RNA-Pol II is disrupted by CHD1 depletion which cause a low occupancy of nucleosomes, as it was observed in yeast (Lee et al., 2012a).

Alternatively, CHD1 might aid the incorporation or removal of H2A.Z during the +1 nucleosome remodeling and thereby decrease H2A.Z levels specifically. It already had been shown that CHD1 was required for the incorporation of histone variants H3.3 and CENP-H into nucleosomes (Konev et al., 2007; Okada et al., 2009). Performing ChIP-seq of $\mathrm{H} 3$ or $\mathrm{H} 2 \mathrm{~B}$ is therefore necessary to rule-out the possibility of less nucleosome occupancy upon CHD1 depletion and can emphasize on the effect of H2A.Z occupancy. Interestingly the histone variant H3.3 commonly co-occupies H2A.Z nucleosomes which further destabilizes them (Jin et al., 2009). Thus, it could be hypothesized that $\mathrm{CHD} 1$ may be required for $\mathrm{H} 3.3$ specific incorporations into nucleosomes at TSS regions too. Therefore, performing H3.3 ChIP at TSS-specific regions upon CHD1 depletion would further improve our knowledge on the role of $\mathrm{CHD} 1$ as a chromatin remodeler in incorporating histone variants. 


\subsubsection{H2Bub1 and CHD1}

Previous studies showed that CHD1 was required for the maintenance of global H2Bub1 levels (Lee et al., 2012a). H2Bub1 was shown to be necessary for MSC differentiation to osteoblast and adipocytes (Karpiuk et al., 2012). Thus, we hypothesized that the differentiation defects observed in our study after CHD1 depletion were due to reduced H2Bub1 levels. However, in our system, we did not see any regulation in H2Bub1 levels upon CHD1 depletion, neither in MSC nor in FOB which might be due to the usage of different cell lines. Lee et al. used yeast and human 293 cells for their studies, but not stem cells. Probably, high levels of H2Bub1 need to be established prior to observe CHD1-specific effects on H2Bub1. Karpiuk et al. showed that undifferentiated MSC possess very negligible amount of H2Bub1 and they gain H2Bub1 during differentiation (Karpiuk et al., 2012). Thus, it could be interesting to test if $\mathrm{CHD} 1$ affects $\mathrm{H} 2 \mathrm{Bub} 1$ stronger in already differentiated cells than during differentiation. Moreover, CHD2, which is also an ATPase-dependent chromatin remodeler and structurally resembles CHD1, may have redundant functions as CHD1 in MSC and FOB, but were not expressed in human 293 cells (Siggens et al., 2015).

\subsection{Genome-wide binding pattern of CHD1}

Several studies and the ENCODE consortium performed ChIP-seq on CHD1 in yeast or human (ENCODE Project Consortium, 2012; Lee et al., 2012a; Siggens et al., 2015). However, comparison of dynamic binding patterns of CHD1 before and after stimulation is poorly understood. Here, we provide a comprehensive overview of CHD1 binding in undifferentiated MSC and after adipocyte and osteoblast differentiation. The observed CHD1 binding regions may possess the following possible regulatory mechanisms:

\subsubsection{CHD1 regulation around TSS}

CHD1 has a broad binding pattern over gene bodies and might act in several ways on transcriptional processes, however its main regulatory role in mammals was thought to be at TSS (Gaspar-Maia et al., 2009; Siggens et al., 2015; Skene et al., 2014). Indeed, in our study, we observed the highest CHD1 binding intensity closely downstream to TSS. Furthermore, we showed that CHD1 binding around TSS positively correlated with gene expression levels and active histone modifications H3K4me3 and H3K27ac. Previous studies revealed similar correlations between 
actively transcribed genes and CHD1 binding at their TSS regions, but categorized the gene expression status quite broad by active, inactive or modestly transcribed sets (Siggens et al., 2015). Here we sought to extend this information and showed for each gene the respective CHD1 binding downstream of its TSS. Especially in osteoblast differentiated MSC, we observed that low and medium expressed genes had high CHD1 binding around their TSS. Furthermore, we could measure that genes induced during differentiation had higher binding of CHD1 which was required for their activation. Altogether, our study demonstrates a strong mechanistic insight connecting the occupancy of CHD1 around TSS and the activity of differentiationinduced genes.

\subsubsection{How is CHD1 recruited to differentiation-induced genes?}

Interaction of CHD1 with transcription-associated complexes like FACT, SAGA or mediator were described before and this could suggest different possibilities for recruitment of CHD1 to the chromatin (Lin et al., 2011; Pray-Grant et al., 2005; Simic et al., 2003). CHD1 may possess differential binding affinities to various complexes. Indeed, strong interaction between CHD1 and Mediator Complex Subunit-1 (MED1) was observed (Lin et al., 2011). Furthermore, another strong interaction was observed between CHD1 and the General Transcription Factor IIH (TFIIH) complex, which is opening up the DNA at the TSS (Guzmán and Lis, 1999). Interestingly, TFIIH is involved in nuclear receptor-associated binding and modulating its activity in gene expression (Compe and Egly, 2012). It was shown to be interacting with RAR, PPARG, AR or the vitamin D receptor (VDR) which also play fundamental roles in regulating differentiation-related gene expression (Jeong and Mangelsdorf, 2009). This altogether indicates that CHD1 may be recruited differentially to TSS by their interaction partners TFIIH or MED1 which can modulate its binding affinity to TSS (Esnault et al., 2008; Mizuta et al., 2014). Performing ChIP-seq on MED1, TFIIH subunits or the nuclear receptors during MSC differentiation can help in understanding the correlation in their binding with CHD1 and looking at CHD1 occupancy upon depletion of these factors would aid us in elucidating the mechanism of $\mathrm{CHD} 1$ recruitment to the chromatin.

\subsubsection{When is CHD1 recruited to differentiation-induced genes?}

A large part of primed and inactive genes in human ESC are however often marked with H3K4me3 (Guenther et al., 2007). These genes are related to differentiation 
processes and get activated during differentiation. In our study, we observed that high levels of CHD1 are positively correlated with high levels of H3K4me3 at the TSS. In contrast, TSS highly marked with H3K4me3 are not necessarily correlated with CHD1 binding. One could speculate that these H3K4me3-enriched genes which are exclusive of CHD1 might be primed for differentiation-specific stimulation. Furthermore, these primed genes can be marked bivalent by $\mathrm{H} 3 \mathrm{~K} 4 \mathrm{me} 3$ and H3K27me3 which had been described before to play a role in stem cell differentiation (Bernstein et al., 2006; Voigt et al., 2013). It could be possible that CHD1 gets recruited after primed genes lose H3K27me3 and are activated. The reasons could be: First, CHD1 and H3K27me3 does not co-occupy as observed in mESC (GasparMaia et al., 2009). Second, occupancy of the strong interaction partner of CHD1, MED1 is reduced at bivalent genes which do not possess the binding of Really Interesting New Gene 1b (RING1B), a PRC1 complex member involved in maintenance of bivalency (Lehmann et al., 2012; Lin et al., 2011). Third, CHD1 is more correlated with active mark H3K27ac than H3K4me3, which does not necessarily mark active genes. Nonetheless is a detailed binding analysis between activating transcription factors, histone modifications and CHD1 occupancy necessary to further understand the dynamics in gene activation during differentiation and the part of CHD1 within it.

\subsubsection{Regulatory roles of CHD1 at gene bodies}

Many of the published binding regions of CHD1 in yeast and human are localized within the gene body (ENCODE Project Consortium, 2012; Lee et al., 2012a; Siggens et al., 2015). Here, we performed quantification of genome-wide CHD1-enriched binding regions and could show that the highest proportion were located within the gene and not at the TSS. Although, the signal intensity of CHD1 occupancy is lower compared to TSS, it could have affected the gene expression in various ways:

\subsubsection{CHD1 role in regulation of Cryptic transcription}

It is known that CHD1 interacts with the FACT complex in $S$. cerevisiae, D. melanogaster and human to order the nucleosomes after RNA-Pol II passage into a regularly spaced array which represses cryptic transcription (Hennig et al., 2012; Kelley et al., 1999; Lin et al., 2011; Simic et al., 2003; Smolle et al., 2012). However, this function was described only in yeast. Nonetheless we also observed CHD1 binding over the gene body which argues for a similar function. Supporting a 
conserved role in repression of cryptic transcription, two studies in yeast and human showed that a mutation in the ATPase-domain of CHD1 caused a decreased nucleosome density over the gene body which is characteristic for cryptic transcription (Hennig and Fischer, 2013; Hennig et al., 2012; Skene et al., 2014). In our study, we sequenced poly-adenylated mRNA in a non-directional approach and thus could not capture cryptic transcripts, which are not poly-adenylated. To study cryptic transcription, one could use deep sequencing of nascent RNA transcripts of RNA-Pol II which would help to capture even very unstable cryptic transcripts (Churchman and Weissman, 2011). Even though cryptic transcription had only mild effects on global transcriptional changes in CHD1 depleted system, it might act in fine tuning gene expression (Colin et al., 2011). If CHD1 affects cryptic transcription in a lineage-specific manner would be interesting to test.

\subsubsection{CHD1 and splicing}

Besides repression of cryptic transcription, CHD1 is also linked with splicing of premRNA (Sims et al., 2007). Sims et al. showed that CHD1 in human interacts with different components of the small nuclear Ribonucleoprotein 2 (snRNP2) riboprotein complex, a part of the huge spliceosome, and is required to associate the complex to the chromatin. Concordantly, a recent study in yeast reported that a CHD1-depleted strain decreased the nucleosome turnover at $3^{\prime}$ end of introns and CHD1 was thought to be necessary to release RNA-Pol II from $5^{\prime}$ intron-exon boundary which might affect splice site recognition (Jonkers and Lis, 2015; Park et al., 2014).In in our study, we also observed a high proportion of CHD1 binding regions within exons. These observations indicated that CHD1 may be involved in the release of RNA-Pol II of intron-exon boundaries and pre-mRNA splicing in MSC and FOB. Deep, strandspecific RNA-seq under CHD1-depleted conditions could help to further elucidate this question by detection of alternatively spliced transcripts (Mills et al., 2013).

Furthermore, one could speculate that less nucleosome occupancy over the gene body as observed in CHD1 mutant cells affect RNA-Pol II traveling speed over the gene which was described to regulate alternative splicing events (Naftelberg et al., 2015; Skene et al., 2014). Additionally to RNA-Pol II traveling speed also the nucleosome distribution at intron-exon boundaries regulate the recognition of splice sites which is also important for alternative spliced mRNA (lannone et al., 2015; Schwartz et al., 2009; Tilgner et al., 2009). Interestingly, alternative splicing is 
involved in osteoblast differentiation as osteoblast differentiation factors like FosB or Lef1 are alternatively spliced and cause differential regulation (Jensen et al., 2010). Moreover, alternatively spliced isoforms of RUNX2 regulate BGLAP expression in different ways, which could be one explanation for the observed differentially expression of BGLAP in MSC and FOB (Makita et al., 2008).

\subsubsection{CHD1 around TTS}

Additional to the prominent peak of CHD1 at the TSS, we observed a second smaller peak closely downstream at the transcriptional termination site (TTS). Even though no studies in metazoans are known in this regard, a study in $S$. pombe showed that the CHD1 homologue hrp1 acts as a transcriptional termination factor (Alén et al., 2002). Interestingly, another recent study linked $\mathrm{CHD} 1$ to the $\mathrm{H} 3$ histone dynamics at the 3 '-end of long genes in S. cerevisiae (Radman-Livaja et al., 2012). We also observed that long genes were significantly less expressed in CHD1-depleted conditions in MSC, but not in FOB (data not shown). Regulation of transcriptional termination by CHD1 is poorly understood. Nevertheless, CHD1 is involved in nucleosome positioning and might help to maintain a nucleosome free region at the TTS which could support the disassembly of RNA-Pol II from the DNA for mRNA cleavage (Core et al., 2008; Mavrich et al., 2008). Furthermore, CHD1 may also be involved in nucleosome turnover at TTS other than TSS (Materne et al.; RadmanLivaja et al., 2012).

\subsubsection{CHD1 at enhancer}

Recently, CHD1 binding was described at enhancer regions which were marked by different H3K4me1/2/3 methylation patterns (Siggens et al., 2015). Indeed, CHD1 also binds to H3K4me2, a mark which is present at active enhancers (Sims et al., 2005; Wang et al., 2014). Although we did not focus on enhancer regions in our study, we detected only a small portion of CHD1 binding at intergenic regions, in MSC as well as FOB. However, mapping of H2A.Z and RNA-Pol II on active enhancers in FOB showed no obvious changes upon CHD1 depletion, though occupancy of both proteins were affected by CHD1 depletion around TSS and play pivotal roles in the regulation of enhancer activity (Brunelle et al., 2015). This indicates that the effects of CHD1 may be negligible at enhancers in our system. Furthermore, we observed a small increase of intergenic bound regions in differentiated cells compared to undifferentiated conditions, which could reflect higher 
binding on enhancers during differentiation. Moreover, mapping CHD1 binding to H3K4me1 and H3K27ac sites which are exclusive of H3K4me3 could improve its binding properties on active enhancer regions.

\subsection{Biological implications on osteoblast differentiation upon CHD1 depletion}

Transcription factors, histone modifying enzymes and chromatin remodeler are important for a rapid transcriptional response to intra- and extracellular signals. During the early and late osteogenesis showed differentiation associated genes a high dynamicity in transcription (Kulterer et al., 2007; Twine et al., 2014). For example, fluctuated the expression of RUNX2 or BGLAP during differentiation, however their dynamic regulation is so far only poorly understood. Nonetheless it is important that this coordinated interplay is maintained and that the factors are expressed at the right time, as one factor can enhance or block differentiation, depending on the differentiation status of a cell (Kahler and Westendorf, 2003; Kahler et al., 2006; Regard et al., 2012). CHD1 likely plays a role in stabilizing this coordinated process by supporting the transcription of fast activated genes as discussed above.

\subsubsection{CHD1 regulation of bone development associated gene ontology terms}

During osteoblast differentiation the extracellular matrix (ECM) is reorganized and organic substrates mainly collagen are deposit on the cell surface (Clarke, 2008). The modification of the ECM is not only a consequence of differentiation but can also affect osteoblast differentiation (Mathews et al., 2012; Xiao et al., 2002). In this study we observed that the highest enrichment of deregulated genes was associated with parts of the ECM. Further, the ECM proteins BGLAP and ELN were one of the strongest deregulated genes in CHD1 depleted conditions. Besides our also another study highlighted the importance of the ECM in transcriptome wide regulation during early osteoblast differentiation (Kulterer et al., 2007). Furthermore, also secreted factors are included in the extracellular region part which were dependent on CHD1 for a normal regulation. More recently, secreted factors were shown to support the coordination of osteoblast differentiation and interestingly were also connected to a systemic signaling (Mrak et al., 2007; Oury et al., 2013; Sabek et al., 2015; Zhong et al., 2012). This together shows that the genes regulated by CHD1 during differentiation might impair osteogenesis also over indirect pathways and not by renowned transcription factors. 
Besides the ECM, deregulated genes were also significantly enriched for the biological processes such as vascularization and blood vessel development. Several studies had implicated a role of MSC signaling on HSC differentiation and development of the microvascular environment in the bone (Deckers et al., 2002; Eshkar-Oren et al., 2009; Furumatsu et al., 2003). These reports were supported by a recent finding of the MSC stem cell niche which resides together with HSC and is located perivascular in direct contact with the blood vessels (Méndez-Ferrer et al., 2010). In sum this implies that applications in vivo could consider the effects of CHD1 on vascularization in particular in cell therapeutic approaches (further discussed below).

\subsubsection{Ectopic bone formation and effects in vivo based on CHD1}

Osteoblastic differentiation consist in general of three phases: proliferation, extracellular matrix organization and subsequent mineralization of this extracellular scaffold (Lian and Stein, 1992). Phenotypically matrix reorganization begins around 5 days after differentiation and includes deposition of organic compounds mainly consisting of different types of collagen (Broek et al., 1985; Kulterer et al., 2007; Niyibizi and Eyre, 1989; Pihlajaniemi et al., 1990). In this study the ectopic bone formation experiment showed low deposition of extracellular matrix in CHD1 depleted conditions. This was concordant with the deregulated genes identified in our RNAseq study which were enriched for terms in ECM organization. Further the observation supports the assumption of an osteoblast differentiation defect before day 5 .

Recent studies described a group of genes predicting a strong ectopic bone formation in mice (Larsen et al., 2010; Twine et al., 2014). Surprisingly, the bona fide osteoblast marker genes $A L P L$ and BGLAP were not specified within this group characteristic for enhanced ectopic bone formation (Larsen et al., 2010). Of particular interest for us was that ELN turned out to be one of the best predictors for ectopic bone formation, which was directly regulated by CHD1. The mechanism of how ELN is acting on bone formation is unknown, however it is expressed during craniofacial bone development in mice and can be induced by TGF $\beta$, which plays an essential role in osteoblast differentiation (Diez-Roux et al., 2011; Sethi et al., 2011). In other tissues ELN was shown to serve as a scaffold protein for mineralization of the ECM which could be transferred to osteoblasts too (Seligman et al., 1975; Starcher and 
Urry, 1973). Further studies will be necessary to unravel its importance in bone formation and if the strong regulatory effect of CHD1 on ELN expression also holds true in other systems.

\subsubsection{Expression of ALPL and BGLAP in osteogenesis after CHD1 depletion}

ALPL and BGLAP are well established marker for osteoblast differentiation. In this study ALPL was higher expressed in MSC but only slightly affected in FOB after CHD1 depletion. Though we observed that the TSS region of ALPL was lowly bound by $\mathrm{CHD} 1$ which indicates an indirect regulation by CHD1 depletion. Thus probably the cellular background of MSC and FOB could explain the observed differences in gene regulation. It could further be speculated that the cells are at a different stage of osteoblast differentiation which is supported by absent expression of BGLAP in FOBs.

Interestingly $A L P L$ and $B G L A P$ are both coordinative activated by the retinoblastoma protein 1 (pRB1) and E2F transcription factor 1 (E2F1) (Flowers et al., 2013). Our RNA-seq study revealed that E2F1 is only lowly expressed in FOB osteoblast differentiated cells, but expressed at an average level in MSC (data not shown). Although MSC and FOB can be both differentiated to osteoblast the different cellular background shown by BGLAP or E2F1 expression indicate that osteogenesis might be triggered in various ways.

\subsection{Translation for clinical therapeutic approaches}

Worldwide currently 320 open clinical studies exist which include mesenchymal stem cells (ClinicalTrial.gov). MSC had been used now for over a decade in cell therapies because of their regenerative and immunomodulatory properties as well as their good safety record in humans (Lepperdinger et al., 2008; Zaher et al., 2014). Besides therapeutic studies in non-skeletal tissues and treatment of immune diseases their regenerative properties were already successfully used for the regeneration of bone and cartilage in human (Gangji et al., 2005; Le Blanc et al., 2005; Quarto et al., 2001; Wakitani et al., 2007). However the repair of skeletal or cartilage depends on the ability of the injected cells to differentiate and form new tissue (Undale et al., 2009). This makes it essential to understand their regenerative and differentiation potential before injection. Though, the canonical osteoblast markers do not predict accurately the in vivo bone formation (Kuznetsov et al 1997, Larsen KH et al 2010). Here we 
suggest with CHD1 another marker which could help to better predict the regenerative potential for bone tissue and maybe cartilage repair.

In cell therapy MSC are commonly expanded ex vivo before transplantation to obtain sufficient material for regenerative purposes (Undale et al., 2009). For example, 1 - 2 million cells per kilogram of body weight are required for a systemic cell therapeutic approach (Schallmoser et al., 2008). A challenge during this culturing ex vivo is to maintain the stem cell characteristics and probably their epigenetic profile ( $\mathrm{Ng}$ et al., 2014). In this study we showed that CHD1 depletion decreased the differentiation potential and was required for broad introduction of transcriptional changes. These properties might be used to decrease the differentiation ex vivo during cell expansion. To my knowledge no small inhibitors of CHD1 exist, however transient siRNA mediated depletion of CHD1 could be applied during ex vivo culturing. Nonetheless deeper investigations especially on transcriptional effects in the undifferentiated state and on the epigenome by CHD1 will unravel its use for therapeutic approaches.

In summary this work reveals a novel regulatory function of $\mathrm{CHD} 1$ in gene activation during osteoblast differentiation and shows its necessity for ectopic bone formation. Markers for safe cell therapy are still poorly described, we suggest to consider CHD1 as one. Further we extend the mechanistic role of CHD1 on global RNA-Pol II stalling around TSS in humans and highlight its requirement for differentiation specific gene activation. Moreover, a new regulatory connection of CHD1 associated H2A.Z incorporation into the +1 nucleosome is hypothesized. Further insights will help to understand if inhibition of CHD1 or H2A.Z are potential targets in stem cell therapy to selectively block differentiation. 


\section{Reference List}

Abbott, D.W., Ivanova, V.S., Wang, X., Bonner, W.M., and Ausió, J. (2001). Characterization of the Stability and Folding of H2A.Z Chromatin Particles Implications for transcriptional activation. J. Biol. Chem. 276, 41945-41949.

Abdallah, B.M., and Kassem, M. (2012). New factors controlling the balance between osteoblastogenesis and adipogenesis. Bone 50, 540-545.

Abdallah, B.M., Ditzel, N., and Kassem, M. (2008). Assessment of bone formation capacity using in vivo transplantation assays: procedure and tissue analysis.

Methods Mol. Biol. Clifton NJ 455, 89-100.

Ahmad, K., and Henikoff, S. (2002). The Histone Variant H3.3 Marks Active Chromatin by Replication-Independent Nucleosome Assembly. Mol. Cell 9, 11911200.

Ahmadian, M., Suh, J.M., Hah, N., Liddle, C., Atkins, A.R., Downes, M., and Evans, R.M. (2013). PPARy signaling and metabolism: the good, the bad and the future. Nat. Med. 99, 557-566.

Albert, I., Mavrich, T.N., Tomsho, L.P., Qi, J., Zanton, S.J., Schuster, S.C., and Pugh, B.F. (2007). Translational and rotational settings of H2A.Z nucleosomes across the Saccharomyces cerevisiae genome. Nature 446, 572-576.

Alberts B, Johnson A, Lewis J, et al. (2002). Chromosomal DNA and Its Packaging in the Chromatin Fiber. Mol. Biol. Cell N. Y. Garland Sci. 4th edition.

Alén, C., Kent, N.A., Jones, H.S., O'Sullivan, J., Aranda, A., and Proudfoot, N.J. (2002). A Role for Chromatin Remodeling in Transcriptional Termination by RNA Polymerase II. Mol. Cell 10, 1441-1452.

Allan, J., Mitchell, T., Harborne, N., Bohm, L., and Crane-Robinson, C. (1986). Roles of $\mathrm{H} 1$ domains in determining higher order chromatin structure and $\mathrm{H} 1$ location. $\mathrm{J}$. Mol. Biol. 187, 591-601.

Al-Nbaheen, M., Vishnubalaji, R., Ali, D., Bouslimi, A., Al-Jassir, F., Megges, M., Prigione, A., Adjaye, J., Kassem, M., and Aldahmash, A. (2012). Human Stromal (Mesenchymal) Stem Cells from Bone Marrow, Adipose Tissue and Skin Exhibit Differences in Molecular Phenotype and Differentiation Potential. Stem Cell Rev. Rep. 9, 32-43.

Álvarez-Errico, D., Vento-Tormo, R., Sieweke, M., and Ballestar, E. (2015).

Epigenetic control of myeloid cell differentiation, identity and function. Nat. Rev. Immunol. 15, 7-17.

Amado, L.C., Saliaris, A.P., Schuleri, K.H., St John, M., Xie, J.-S., Cattaneo, S., Durand, D.J., Fitton, T., Kuang, J.Q., Stewart, G., et al. (2005). Cardiac repair with intramyocardial injection of allogeneic mesenchymal stem cells after myocardial infarction. Proc. Natl. Acad. Sci. U. S. A. 102, 11474-11479. 
Anders, S., and Huber, W. (2010). Differential expression analysis for sequence count data. Genome Biol. 11, R106.

Antebi, B., Pelled, G., and Gazit, D. (2014). Stem cell therapy for osteoporosis. Curr. Osteoporos. Rep. 12, 41-47.

Atoui, R., and Chiu, R.C.J. (2012). Concise Review: Immunomodulatory Properties of Mesenchymal Stem Cells in Cellular Transplantation: Update, Controversies, and Unknowns. Stem Cells Transl. Med. 1, 200-205.

Badis, G., Chan, E.T., van Bakel, H., Pena-Castillo, L., Tillo, D., Tsui, K., Carlson, C.D., Gossett, A.J., Hasinoff, M.J., Warren, C.L., et al. (2008). A library of yeast transcription factor motifs reveals a widespread function for Rsc3 in targeting nucleosome exclusion at promoters. Mol. Cell 32, 878-887.

Banaszynski, L.A., Wen, D., Dewell, S., Whitcomb, S.J., Lin, M., Diaz, N., Elsässer, S.J., Chapgier, A., Goldberg, A.D., Canaani, E., et al. (2013). Hira-dependent histone H3.3 deposition facilitates PRC2 recruitment at developmental loci in ES cells. Cell $155,107-120$.

Bannister, A.J., and Kouzarides, T. (2011). Regulation of chromatin by histone modifications. Cell Res. 21, 381-395.

Barth, T.K., and Imhof, A. (2010). Fast signals and slow marks: the dynamics of histone modifications. Trends Biochem. Sci. 35, 618-626.

Bernstein, B.E., Mikkelsen, T.S., Xie, X., Kamal, M., Huebert, D.J., Cuff, J., Fry, B., Meissner, A., Wernig, M., Plath, K., et al. (2006). A bivalent chromatin structure marks key developmental genes in embryonic stem cells. Cell 125, 315-326.

Bernstein, B.E., Stamatoyannopoulos, J.A., Costello, J.F., Ren, B., Milosavljevic, A., Meissner, A., Kellis, M., Marra, M.A., Beaudet, A.L., Ecker, J.R., et al. (2010). The NIH Roadmap Epigenomics Mapping Consortium. Nat. Biotechnol. 28, 1045-1048.

Bianco, P., and Robey, P.G. (2015). Skeletal stem cells. Development 142, $1023-$ 1027.

Billon, P., and Côté, J. (2013). Precise deposition of histone H2A.Z in chromatin for genome expression and maintenance. Biochim. Biophys. Acta 1819, 290-302.

de Boer, J., Siddappa, R., Gaspar, C., van Apeldoorn, A., Fodde, R., and van Blitterswijk, C. (2004). Wnt signaling inhibits osteogenic differentiation of human mesenchymal stem cells. Bone 34, 818-826.

Boland, G.M., Perkins, G., Hall, D.J., and Tuan, R.S. (2004). Wnt 3a promotes proliferation and suppresses osteogenic differentiation of adult human mesenchymal stem cells. J. Cell. Biochem. 93, 1210-1230.

Bönisch, C., and Hake, S.B. (2012). Histone H2A variants in nucleosomes and chromatin: more or less stable? Nucleic Acids Res. 40, 10719-10741.

Broek, D.L., Madri, J., Eikenberry, E.F., and Brodsky, B. (1985). Characterization of the tissue form of type V collagen from chick bone. J. Biol. Chem. 260, 555-562. 
Brunelle, M., Nordell Markovits, A., Rodrigue, S., Lupien, M., Jacques, P.-É., and Gévry, N. (2015). The histone variant H2A.Z is an important regulator of enhancer activity. Nucleic Acids Res. 43, 9742-9756.

Bugga, L., McDaniel, I.E., Engie, L., and Armstrong, J.A. (2013). The Drosophila melanogaster CHD1 Chromatin Remodeling Factor Modulates Global Chromosome Structure and Counteracts HP1a and H3K9me2. PLoS ONE 8, e59496.

Burkhardt, L., Fuchs, S., Krohn, A., Masser, S., Mader, M., Kluth, M., Bachmann, F., Huland, H., Steuber, T., Graefen, M., et al. (2013). CHD1 Is a 5q21 Tumor Suppressor Required for ERG Rearrangement in Prostate Cancer. Cancer Res. 73, 2795-2805.

Cairns, B.R. (2007). Chromatin remodeling: insights and intrigue from singlemolecule studies. Nat. Struct. Mol. Biol. 14, 989-996.

Carvalho, S., Raposo, A.C., Martins, F.B., Grosso, A.R., Sridhara, S.C., Rino, J., Carmo-Fonseca, M., and de Almeida, S.F. (2013). Histone methyltransferase SETD2 coordinates FACT recruitment with nucleosome dynamics during transcription. Nucleic Acids Res. 41, 2881-2893.

Chadwick, B.P., and Willard, H.F. (2001). A Novel Chromatin Protein, Distantly Related to Histone $\mathrm{H} 2 \mathrm{a}$, Is Largely Excluded from the Inactive X Chromosome. J. Cell Biol. 152, 375-384.

Chang, C.H., and Luse, D.S. (1997). The H3/H4 tetramer blocks transcript elongation by RNA polymerase II in vitro. J. Biol. Chem. 272, 23427-23434.

Chapel, A., Bertho, J.M., Bensidhoum, M., Fouillard, L., Young, R.G., Frick, J., Demarquay, C., Cuvelier, F., Mathieu, E., Trompier, F., et al. (2003). Mesenchymal stem cells home to injured tissues when co-infused with hematopoietic cells to treat a radiation-induced multi-organ failure syndrome. J. Gene Med. 5, 1028-1038.

Chen, G., Deng, C., and Li, Y.-P. (2012). TGF- $\beta$ and BMP signaling in osteoblast differentiation and bone formation. Int. J. Biol. Sci. 8, 272-288.

Chen, K., Johnston, J., Shao, W., Meier, S., Staber, C., and Zeitlinger, J. (2013). A global change in RNA polymerase II pausing during the Drosophila midblastula transition. eLife 2, e00861.

Chen, M., Qiao, H., Su, Z., Li, H., Ping, Q., and Zong, L. (2014a). Emerging therapeutic targets for osteoporosis treatment. Expert Opin. Ther. Targets 18, 817831.

Chen, P., Wang, Y., and Li, G. (2014b). Dynamics of histone variant $\mathrm{H} 3.3$ and its coregulation with H2A.Z at enhancers and promoters. Nucleus 5, 21-27.

Chen, L. and Ditzel, N. (2015). In vivo Heterotopic Bone Formation Assay Using Isolated Mouse and Human Mesenchymal Stem Cells. Bio-Protoc.

Chi, T.H., Wan, M., Lee, P.P., Akashi, K., Metzger, D., Chambon, P., Wilson, C.B., and Crabtree, G.R. (2003). Sequential roles of Brg, the ATPase subunit of BAF chromatin remodeling complexes, in thymocyte development. Immunity 19, 169-182. 
Cho, I., Tsai, P.-F., Lake, R.J., Basheer, A., and Fan, H.-Y. (2013). ATP-Dependent Chromatin Remodeling by Cockayne Syndrome Protein B and NAP1-Like Histone Chaperones Is Required for Efficient Transcription-Coupled DNA Repair. PLoS Genet 9, e1003407.

Churchman, L.S., and Weissman, J.S. (2011). Nascent transcript sequencing visualizes transcription at nucleotide resolution. Nature 469.

Clapier, C.R., and Cairns, B.R. (2009). The biology of chromatin remodeling complexes. Annu. Rev. Biochem. 78, 273-304.

Clarke, B. (2008). Normal Bone Anatomy and Physiology. Clin. J. Am. Soc. Nephrol. CJASN 3, S131-S139.

Colin, J., Libri, D., and Porrua, O. (2011). Cryptic Transcription and Early Termination in the Control of Gene Expression. Genet. Res. Int. 2011.

Compe, E., and Egly, J.-M. (2012). TFIIH: when transcription met DNA repair. Nat. Rev. Mol. Cell Biol. 13, 343-354.

Cooper, G.M. (2000). Regulation of Transcription in Eukaryotes.

Core, L.J., Waterfall, J.J., and Lis, J.T. (2008). Nascent RNA sequencing reveals widespread pausing and divergent initiation at human promoters. Science 322, 1845-1848.

Costanzi, C., and Pehrson, J.R. (1998). Histone macroH2A1 is concentrated in the inactive X chromosome of female mammals. Nature 393, 599-601.

Damsky, C.H. (1999). Extracellular matrix-integrin interactions in osteoblast function and tissue remodeling. Bone 25, 95-96.

Das, C., and Tyler, J.K. (2013). Histone exchange and histone modifications during transcription and aging. Biochim. Biophys. Acta 1819, 332-342.

Das, A.V., James, J., Bhattacharya, S., Imbalzano, A.N., Antony, M.L., Hegde, G., Zhao, X., Mallya, K., Ahmad, F., Knudsen, E., et al. (2007). SWI/SNF chromatin remodeling ATPase Brm regulates the differentiation of early retinal stem cells/progenitors by influencing Brn3b expression and Notch signaling. J. Biol. Chem. 282, 35187-35201.

Day, T.F., Guo, X., Garrett-Beal, L., and Yang, Y. (2005). Wnt/beta-catenin signaling in mesenchymal progenitors controls osteoblast and chondrocyte differentiation during vertebrate skeletogenesis. Dev. Cell 8, 739-750.

Deckers, M.M.L., van Bezooijen, R.L., van der Horst, G., Hoogendam, J., van Der Bent, C., Papapoulos, S.E., and Löwik, C.W.G.M. (2002). Bone morphogenetic proteins stimulate angiogenesis through osteoblast-derived vascular endothelial growth factor A. Endocrinology 143, 1545-1553.

Diez-Roux, G., Banfi, S., Sultan, M., Geffers, L., Anand, S., Rozado, D., Magen, A., Canidio, E., Pagani, M., Peluso, I., et al. (2011). A high-resolution anatomical atlas of the transcriptome in the mouse embryo. PLoS Biol. 9, e1000582. 
Dutta, D., Ray, S., Home, P., Saha, B., Wang, S., Sheibani, N., Tawfik, O., Cheng, N., and Paul, S. (2010). Regulation of Angiogenesis by Histone Chaperone HIRAmediated Incorporation of Lysine 56-acetylated Histone H3.3 at Chromatin Domains of Endothelial Genes. J. Biol. Chem. 285, 41567-41577.

Eckfeldt, C.E., Mendenhall, E.M., and Verfaillie, C.M. (2005). The molecular repertoire of the "almighty" stem cell. Nat. Rev. Mol. Cell Biol. 6, 726-737.

Eijken, M., Meijer, I.M.J., Westbroek, I., Koedam, M., Chiba, H., Uitterlinden, A.G., Pols, H. a. P., and van Leeuwen, J.P.T.M. (2008). Wnt signaling acts and is regulated in a human osteoblast differentiation dependent manner. J. Cell. Biochem. 104, 568579.

ENCODE Project Consortium (2012). An integrated encyclopedia of DNA elements in the human genome. Nature 489, 57-74.

Epshtein, V., and Nudler, E. (2003). Cooperation Between RNA Polymerase Molecules in Transcription Elongation. Science 300, 801-805.

Eshkar-Oren, I., Viukov, S.V., Salameh, S., Krief, S., Oh, C., Akiyama, H., Gerber, H.-P., Ferrara, N., and Zelzer, E. (2009). The forming limb skeleton serves as a signaling center for limb vasculature patterning via regulation of Vegf. Dev. Camb. Engl. 136, 1263-1272.

Esnault, C., Ghavi-Helm, Y., Brun, S., Soutourina, J., Van Berkum, N., Boschiero, C., Holstege, F., and Werner, M. (2008). Mediator-dependent recruitment of TFIIH modules in preinitiation complex. Mol. Cell 31, 337-346.

Fletcher, T.M., and Hansen, J.C. (1996). The nucleosomal array: structure/function relationships. Crit. Rev. Eukaryot. Gene Expr. 6, 149-188.

Flowers, S., Xu, F., and Moran, E. (2013). Cooperative Activation of Tissue-Specific Genes by pRB and E2F1. Cancer Res. 73, 2150-2158.

Fritah, A., Saucier, C., Mester, J., Redeuilh, G., and Sabbah, M. (2005). p21WAF1/CIP1 Selectively Controls the Transcriptional Activity of Estrogen Receptor a. Mol. Cell. Biol. 25, 2419-2430.

Fuchs, G., Hollander, D., Voichek, Y., Ast, G., and Oren, M. (2014). Cotranscriptional histone $\mathrm{H} 2 \mathrm{~B}$ monoubiquitylation is tightly coupled with RNA polymerase II elongation rate. Genome Res. 24, 1572-1583.

Furumatsu, T., Shen, Z.N., Kawai, A., Nishida, K., Manabe, H., Oohashi, T., Inoue, H., and Ninomiya, Y. (2003). Vascular endothelial growth factor principally acts as the main angiogenic factor in the early stage of human osteoblastogenesis. J. Biochem. (Tokyo) 133, 633-639.

Gaffney, D.J., McVicker, G., Pai, A.A., Fondufe-Mittendorf, Y.N., Lewellen, N., Michelini, K., Widom, J., Gilad, Y., and Pritchard, J.K. (2012). Controls of Nucleosome Positioning in the Human Genome. PLoS Genet 8, e1003036.

Gama, A., Navet, B., Vargas, J.W., Castaneda, B., and Lézot, F. (2015). Bone resorption: an actor of dental and periodontal development? Craniofacial Biol. 319. 
Gangji, V., Toungouz, M., and Hauzeur, J.-P. (2005). Stem cell therapy for osteonecrosis of the femoral head. Expert Opin. Biol. Ther. 5, 437-442.

Gao, H., Lukin, K., Ramírez, J., Fields, S., Lopez, D., and Hagman, J. (2009). Opposing effects of SWI/SNF and Mi-2/NuRD chromatin remodeling complexes on epigenetic reprogramming by EBF and Pax5. Proc. Natl. Acad. Sci. U. S. A. 106, 11258-11263.

Gaspar-Maia, A., Alajem, A., Polesso, F., Sridharan, R., Mason, M.J., Heidersbach, A., Ramalho-Santos, J., McManus, M.T., Plath, K., Meshorer, E., et al. (2009). Chd1 regulates open chromatin and pluripotency of embryonic stem cells. Nature 460 , 863-868.

Gelman, L., Zhou, G., Fajas, L., Raspé, E., Fruchart, J.C., and Auwerx, J. (1999). p300 interacts with the N- and C-terminal part of PPARgamma2 in a ligandindependent and -dependent manner, respectively. J. Biol. Chem. 274, 7681-7688.

Gkikopoulos, T., Schofield, P., Singh, V., Pinskaya, M., Mellor, J., Smolle, M., Workman, J.L., Barton, G.J., and Owen-Hughes, T. (2011). A role for Snf2-related nucleosome-spacing enzymes in genome-wide nucleosome organization. Science 333, 1758-1760.

Gomes, N.P., Bjerke, G., Llorente, B., Szostek, S.A., Emerson, B.M., and Espinosa, J.M. (2006). Gene-specific requirement for P-TEFb activity and RNA polymerase II phosphorylation within the p53 transcriptional program. Genes Dev. 20, 601-612.

Gómez-Barrena, E., Rosset, P., Lozano, D., Stanovici, J., Ermthaller, C., and Gerbhard, F. (2015). Bone fracture healing: Cell therapy in delayed unions and nonunions. Bone 70, 93-101.

Grandy, R.A., Whitfield, T.W., Wu, H., Fitzgerald, M.P., VanOudenhove, J.J., Zaidi, S.K., Montecino, M.A., Lian, J.B., vanWijnen, A.J., Stein, J.L., et al. (2015). Genomewide Studies Reveal that H3K4me3 Modification in Bivalent Genes is Dynamically Regulated During the Pluripotent Cell Cycle and Stabilized Upon Differentiation. Mol. Cell. Biol.

Grasso, C.S., Wu, Y.-M., Robinson, D.R., Cao, X., Dhanasekaran, S.M., Khan, A.P., Quist, M.J., Jing, X., Lonigro, R.J., Brenner, J.C., et al. (2012). The mutational landscape of lethal castration-resistant prostate cancer. Nature 487, 239-243.

Green, C.M., and Almouzni, G. (2002). When repair meets chromatin. First in series on chromatin dynamics. EMBO Rep. 3, 28-33.

Grewal, S.I.S., and Jia, S. (2007). Heterochromatin revisited. Nat. Rev. Genet. 8, 3546.

Guenther, M.G., Levine, S.S., Boyer, L.A., Jaenisch, R., and Young, R.A. (2007). A Chromatin Landmark and Transcription Initiation at Most Promoters in Human Cells. Cell 130, 77-88. 
Guillemette, B., Bataille, A.R., Gévry, N., Adam, M., Blanchette, M., Robert, F., and Gaudreau, L. (2005). Variant Histone H2A.Z Is Globally Localized to the Promoters of Inactive Yeast Genes and Regulates Nucleosome Positioning. PLoS Biol 3, e384.

Gurard-Levin, Z.A., Quivy, J.-P., and Almouzni, G. (2014). Histone Chaperones: Assisting Histone Traffic and Nucleosome Dynamics. Annu. Rev. Biochem. 83, 487517.

Guzmán, E., and Lis, J.T. (1999). Transcription factor TFIIH is required for promoter melting in vivo. Mol. Cell. Biol. 19, 5652-5658.

Guzman-Ayala, M., Sachs, M., Koh, F.M., Onodera, C., Bulut-Karslioglu, A., Lin, C.J., Wong, P., Nitta, R., Song, J.S., and Ramalho-Santos, M. (2015). Chd1 is essential for the high transcriptional output and rapid growth of the mouse epiblast.

Development 142, 118-127.

Hansen, J.C. (2002). Conformational Dynamics of the Chromatin Fiber in Solution: Determinants, Mechanisms, and Functions. Annu. Rev. Biophys. Biomol. Struct. 31, 361-392.

Harris, S.A., Enger, R.J., Riggs, L.B., and Spelsberg, T.C. (1995). Development and characterization of a conditionally immortalized human fetal osteoblastic cell line. J. Bone Miner. Res. 10, 178-186.

Hauk, G., McKnight, J.N., Nodelman, I.M., and Bowman, G.D. (2010). The Chromodomains of the Chd1 Chromatin Remodeler Regulate DNA Access to the ATPase Motor. Mol. Cell 39, 711-723.

Heard, E., and Disteche, C.M. (2006). Dosage compensation in mammals: finetuning the expression of the X chromosome. Genes Dev. 20, 1848-1867.

Heintzman, N.D., Hon, G.C., Hawkins, R.D., Kheradpour, P., Stark, A., Harp, L.F., Ye, Z., Lee, L.K., Stuart, R.K., Ching, C.W., et al. (2009). Histone Modifications at Human Enhancers Reflect Global Cell Type-Specific Gene Expression. Nature 459, $108-112$.

Heinz, S., Romanoski, C.E., Benner, C., and Glass, C.K. (2015). The selection and function of cell type-specific enhancers. Nat. Rev. Mol. Cell Biol. 16, 144-154.

Henikoff, S., Henikoff, J.G., Sakai, A., Loeb, G.B., and Ahmad, K. (2009). Genomewide profiling of salt fractions maps physical properties of chromatin. Genome Res. 19, 460-469.

Hennig, B.P., and Fischer, T. (2013). The great repression. Transcription 4, 97-101.

Hennig, B.P., Bendrin, K., Zhou, Y., and Fischer, T. (2012). Chd1 chromatin remodelers maintain nucleosome organization and repress cryptic transcription. EMBO Rep. 13, 997-1003.

Hill, T.P., Später, D., Taketo, M.M., Birchmeier, W., and Hartmann, C. (2005). Canonical Wnt/ $\beta$-Catenin Signaling Prevents Osteoblasts from Differentiating into Chondrocytes. Dev. Cell 8, 727-738. 
Hoch, A.I., and Leach, J.K. (2014). Concise Review: Optimizing Expansion of Bone Marrow Mesenchymal Stem/Stromal Cells for Clinical Applications. Stem Cells Transl. Med. 3, 643-652.

Hödl, M., and Basler, K. (2012). Transcription in the absence of histone H3.2 and H3K4 methylation. Curr. Biol. CB 22, 2253-2257.

Hsieh, F.-K., Kulaeva, O.I., Patel, S.S., Dyer, P.N., Luger, K., Reinberg, D., and Studitsky, V.M. (2013). Histone chaperone FACT action during transcription through chromatin by RNA polymerase II. Proc. Natl. Acad. Sci. U. S. A. 110, 7654-7659.

Hu, H., Hilton, M.J., Tu, X., Yu, K., Ornitz, D.M., and Long, F. (2005). Sequential roles of Hedgehog and Wnt signaling in osteoblast development. Dev. Camb. Engl. 132, 49-60.

Huang, D.W., Sherman, B.T., and Lempicki, R.A. (2009). Bioinformatics enrichment tools: paths toward the comprehensive functional analysis of large gene lists. Nucleic Acids Res. 37, 1-13.

Huang, S., Gulzar, Z.G., Salari, K., Lapointe, J., Brooks, J.D., and Pollack, J.R. (2012). Recurrent deletion of CHD1 in prostate cancer with relevance to cell invasiveness. Oncogene 31, 4164-4170.

lannone, C., Pohl, A., Papasaikas, P., Soronellas, D., Vicent, G.P., Beato, M., and ValcáRcel, J. (2015). Relationship between nucleosome positioning and progesterone-induced alternative splicing in breast cancer cells. RNA N. Y. N 21, 360-374.

louzalen, N., Moreau, J., and Méchali, M. (1996). H2A.ZI, a New Variant Histone Expressed during Xenopus Early Development Exhibits Several Distinct Features from the Core Histone H2A. Nucleic Acids Res. 24, 3947-3952.

Jaenisch, R., and Bird, A. (2003). Epigenetic regulation of gene expression: how the genome integrates intrinsic and environmental signals. Nat. Genet. 33, 245-254.

Jensen, E.D., Gopalakrishnan, R., and Westendorf, J.J. (2010). Regulation of Gene Expression in Osteoblasts. BioFactors Oxf. Engl. 36, 25-32.

Jeong, Y., and Mangelsdorf, D.J. (2009). Nuclear receptor regulation of stemness and stem cell differentiation. Exp. Mol. Med. 41, 525-537.

Jimeno-González, S., Gómez-Herreros, F., Alepuz, P.M., and Chávez, S. (2006). A Gene-Specific Requirement for FACT during Transcription Is Related to the Chromatin Organization of the Transcribed Region. Mol. Cell. Biol. 26, 8710-8721.

Jin, C., and Felsenfeld, G. (2007). Nucleosome stability mediated by histone variants H3.3 and H2A.Z. Genes Dev. 21, 1519-1529.

Jin, C., Zang, C., Wei, G., Cui, K., Peng, W., Zhao, K., and Felsenfeld, G. (2009). H3.3/H2A.Z double variant-containing nucleosomes mark "nucleosome-free regions" of active promoters and other regulatory regions. Nat. Genet. 41, 941-945. 
Jonkers, I., and Lis, J.T. (2015). Getting up to speed with transcription elongation by RNA polymerase II. Nat. Rev. Mol. Cell Biol. 16, 167-177.

Kahler, R.A., and Westendorf, J.J. (2003). Lymphoid enhancer factor-1 and betacatenin inhibit Runx2-dependent transcriptional activation of the osteocalcin promoter. J. Biol. Chem. 278, 11937-11944.

Kahler, R.A., Galindo, M., Lian, J., Stein, G.S., van Wijnen, A.J., and Westendorf, J.J. (2006). Lymphocyte enhancer-binding factor 1 (Lef1) inhibits terminal differentiation of osteoblasts. J. Cell. Biochem. 97, 969-983.

Kahler, R.A., Yingst, S.M.C., Hoeppner, L.H., Jensen, E.D., Krawczak, D., Oxford, J.T., and Westendorf, J.J. (2008). Collagen $11 \mathrm{a} 1$ is indirectly activated by lymphocyte enhancer-binding factor 1 (Lef1) and negatively regulates osteoblast maturation. Matrix Biol. J. Int. Soc. Matrix Biol. 27, 330-338.

Kamakaka, R.T., and Biggins, S. (2005). Histone variants: deviants? Genes Dev. 19, 295-316.

Kamakaka, R.T., and Thomas, J.O. (1990). Chromatin structure of transcriptionally competent and repressed genes. EMBO J. 9, 3997-4006.

Kanatani, N., Fujita, T., Fukuyama, R., Liu, W., Yoshida, C.A., Moriishi, T., Yamana, K., Miyazaki, T., Toyosawa, S., and Komori, T. (2006). Cbf beta regulates Runx2 function isoform-dependently in postnatal bone development. Dev. Biol. 296, 48-61.

Karlić, R., Chung, H.-R., Lasserre, J., Vlahoviček, K., and Vingron, M. (2010). Histone modification levels are predictive for gene expression. Proc. Natl. Acad. Sci. U. S. A. 107, 2926-2931.

Karpiuk, O., Najafova, Z., Kramer, F., Hennion, M., Galonska, C., König, A., Snaidero, N., Vogel, T., Shchebet, A., Begus-Nahrmann, Y., et al. (2012a). The Histone H2B Monoubiquitination Regulatory Pathway Is Required for Differentiation of Multipotent Stem Cells. Mol. Cell 46, 705-713.

Kassem, M., and Bianco, P. (2015). Skeletal Stem Cells in Space and Time. Cell 160, 17-19.

Kato, S., Inoue, K., and Youn, M.-Y. (2010). Emergence of the osteo-epigenome in bone biology. IBMS BoneKEy 7, 314-324.

Kelley, D.E., Stokes, D.G., and Perry, R.P. (1999). CHD1 interacts with SSRP1 and depends on both its chromodomain and its ATPase/helicase-like domain for proper association with chromatin. Chromosoma 108, 10-25.

Kim, J.-M., Lee, S.-T., Chu, K., Jung, K.-H., Song, E.-C., Kim, S.-J., Sinn, D.-I., Kim, J.-H., Park, D.-K., Kang, K.-M., et al. (2007). Systemic transplantation of human adipose stem cells attenuated cerebral inflammation and degeneration in a hemorrhagic stroke model. Brain Res. 1183, 43-50.

Kireeva, M.L., Walter, W., Tchernajenko, V., Bondarenko, V., Kashlev, M., and Studitsky, V.M. (2002). Nucleosome Remodeling Induced by RNA Polymerase II: Loss of the H2A/H2B Dimer during Transcription. Mol. Cell 9, 541-552. 
Koch, C.M., Andrews, R.M., Flicek, P., Dillon, S.C., Karaöz, U., Clelland, G.K., Wilcox, S., Beare, D.M., Fowler, J.C., Couttet, P., et al. (2007). The landscape of histone modifications across $1 \%$ of the human genome in five human cell lines. Genome Res. 17, 691-707.

Koh, F.M., Lizama, C.O., Wong, P., Hawkins, J.S., Zovein, A.C., and RamalhoSantos, M. (2015). Emergence of hematopoietic stem and progenitor cells involves a Chd1-dependent increase in total nascent transcription. Proc. Natl. Acad. Sci. 112, E1734-E1743.

Komori, T. (2009). Regulation of Osteoblast Differentiation by Runx2. In Osteoimmunology, Y. Choi, ed. (Springer US), pp. 43-49.

Konev, A.Y., Tribus, M., Park, S.Y., Podhraski, V., Lim, C.Y., Emelyanov, A.V., Vershilova, E., Pirrotta, V., Kadonaga, J.T., Lusser, A., et al. (2007). CHD1 motor protein is required for deposition of histone variant $\mathrm{H} 3.3$ into chromatin in vivo. Science 317, 1087-1090.

Kornberg, R.D. (1974). Chromatin structure: a repeating unit of histones and DNA. Science 184, 868-871.

Krogan, N.J., Kim, M., Ahn, S.H., Zhong, G., Kobor, M.S., Cagney, G., Emili, A., Shilatifard, A., Buratowski, S., and Greenblatt, J.F. (2002). RNA Polymerase II Elongation Factors of Saccharomyces cerevisiae: a Targeted Proteomics Approach. Mol. Cell. Biol. 22, 6979-6992.

Ku, M., Jaffe, J.D., Koche, R.P., Rheinbay, E., Endoh, M., Koseki, H., Carr, S.A., and Bernstein, B.E. (2012). H2A.Z landscapes and dual modifications in pluripotent and multipotent stem cells underlie complex genome regulatory functions. Genome Biol. 13, R85.

Kulish, D., and Struhl, K. (2001). TFIIS Enhances Transcriptional Elongation through an Artificial Arrest Site In Vivo. Mol. Cell. Biol. 21, 4162-4168.

Kulterer, B., Friedl, G., Jandrositz, A., Sanchez-Cabo, F., Prokesch, A., Paar, C., Scheideler, M., Windhager, R., Preisegger, K.-H., and Trajanoski, Z. (2007). Gene expression profiling of human mesenchymal stem cells derived from bone marrow during expansion and osteoblast differentiation. BMC Genomics 8, 70.

Kwon, H., Imbalzano, A.N., Khavari, P.A., Kingston, R.E., and Green, M.R. (1994). Nucleosome disruption and enhancement of activator binding by a human SW1/SNF complex. Nature 370, 477-481.

Lam, M.T., Li, W., Rosenfeld, M.G., and Glass, C.K. (2014). Enhancer RNAs and regulated transcriptional programs. Trends Biochem. Sci. 39, 170-182.

Langmead, B., and Salzberg, S.L. (2012). Fast gapped-read alignment with Bowtie 2. Nat. Methods 9, 357-359.

Langmead, B., Trapnell, C., Pop, M., and Salzberg, S.L. (2009). Ultrafast and memory-efficient alignment of short DNA sequences to the human genome. Genome Biol. 10, R25. 
Larsen, K.H., Frederiksen, C.M., Burns, J.S., Abdallah, B.M., and Kassem, M. (2010). Identifying a molecular phenotype for bone marrow stromal cells with in vivo boneforming capacity. J. Bone Miner. Res. 25, 796-808.

Le Blanc, K., Götherström, C., Ringdén, O., Hassan, M., McMahon, R., Horwitz, E., Anneren, G., Axelsson, O., Nunn, J., Ewald, U., et al. (2005). Fetal mesenchymal stem-cell engraftment in bone after in utero transplantation in a patient with severe osteogenesis imperfecta. Transplantation 79, 1607-1614.

Ledoux, S., Queguiner, I., Msika, S., Calderari, S., Rufat, P., Gasc, J.-M., Corvol, P., and Larger, E. (2008). Angiogenesis Associated With Visceral and Subcutaneous Adipose Tissue in Severe Human Obesity. Diabetes 57, 3247-3257.

Lee, J.-S., Garrett, A.S., Yen, K., Takahashi, Y.-H., Hu, D., Jackson, J., Seidel, C., Pugh, B.F., and Shilatifard, A. (2012a). Codependency of H2B monoubiquitination and nucleosome reassembly on Chd1. Genes Dev. 26, 914-919.

Lee, J.W., Fang, X., Gupta, N., Serikov, V., and Matthay, M.A. (2009). Allogeneic human mesenchymal stem cells for treatment of $E$. coli endotoxin-induced acute lung injury in the ex vivo perfused human lung. Proc. Natl. Acad. Sci. U. S. A. 106, 1635716362.

Lee, K.S., Kim, H.J., Li, Q.L., Chi, X.Z., Ueta, C., Komori, T., Wozney, J.M., Kim, E.G., Choi, J.Y., Ryoo, H.M., et al. (2000). Runx2 is a common target of transforming growth factor beta1 and bone morphogenetic protein 2, and cooperation between Runx2 and Smad5 induces osteoblast-specific gene expression in the pluripotent mesenchymal precursor cell line C2C12. Mol. Cell. Biol. 20, 8783-8792.

Lee, M.-H., Kim, Y.-J., Kim, H.-J., Park, H.-D., Kang, A.-R., Kyung, H.-M., Sung, J.H., Wozney, J.M., Kim, H.-J., and Ryoo, H.-M. (2003). BMP-2-induced Runx2 Expression Is Mediated by DIx5, and TGF- $\beta 1$ Opposes the BMP-2-induced Osteoblast Differentiation by Suppression of DIx5 Expression. J. Biol. Chem. 278, 34387-34394.

Lehmann, L., Ferrari, R., Vashisht, A.A., Wohlschlegel, J.A., Kurdistani, S.K., and Carey, M. (2012). Polycomb Repressive Complex 1 (PRC1) Disassembles RNA Polymerase II Preinitiation Complexes». J. Biol. Chem. 287, 35784-35794.

Lepperdinger, G., Brunauer, R., Jamnig, A., Laschober, G., and Kassem, M. (2008). Controversial issue: is it safe to employ mesenchymal stem cells in cell-based therapies? Exp. Gerontol. 43, 1018-1023.

Li, G., and Reinberg, D. (2011). Chromatin higher-order structures and gene regulation. Curr. Opin. Genet. Dev. 21, 175-186.

Li, J., and Gilmour, D.S. (2013). Distinct mechanisms of transcriptional pausing orchestrated by GAGA factor and M1BP, a novel transcription factor. EMBO J. 32, 1829-1841.

Li, B., Carey, M., and Workman, J.L. (2007). The Role of Chromatin during Transcription. Cell 128, 707-719. 
Li, G., Margueron, R., Hu, G., Stokes, D., Wang, Y.-H., and Reinberg, D. (2010). Highly Compacted Chromatin Formed In Vitro Reflects the Dynamics of Transcription Activation In Vivo. Mol. Cell 38, 41-53.

Li, H., Handsaker, B., Wysoker, A., Fennell, T., Ruan, J., Homer, N., Marth, G., Abecasis, G., and Durbin, R. (2009). The Sequence Alignment/Map format and SAMtools. Bioinformatics 25, 2078-2079.

Lian, J.B., and Stein, G.S. (1992). Concepts of Osteoblast Growth and Differentiation: Basis for Modulation of Bone Cell Development and Tissue Formation. Crit. Rev. Oral Biol. Med. 3, 269-305.

Lin, C.Y., Lovén, J., Rahl, P.B., Paranal, R.M., Burge, C.B., Bradner, J.E., Lee, T.I., and Young, R.A. (2012). Transcriptional amplification in tumor cells with elevated cMyc. Cell 151, 56-67.

Lin, J.J., Lehmann, L.W., Bonora, G., Sridharan, R., Vashisht, A.A., Tran, N., Plath, K., Wohlschlegel, J.A., and Carey, M. (2011). Mediator coordinates PIC assembly with recruitment of CHD1. Genes Dev. 25, 2198-2209.

Long, F. (2012). Building strong bones: molecular regulation of the osteoblast lineage. Nat. Rev. Mol. Cell Biol. 13, 27-38.

Lorch, Y., LaPointe, J.W., and Kornberg, R.D. (1987). Nucleosomes inhibit the initiation of transcription but allow chain elongation with the displacement of histones. Cell 49, 203-210.

Luger, K., Mäder, A.W., Richmond, R.K., Sargent, D.F., and Richmond, T.J. (1997). Crystal structure of the nucleosome core particle at $2.8 \AA$ resolution. Nature 389 , 251-260.

Lusser, A., Urwin, D.L., and Kadonaga, J.T. (2005). Distinct activities of CHD1 and ACF in ATP-dependent chromatin assembly. Nat. Struct. Mol. Biol. 12, 160-166.

Makita, N., Suzuki, M., Asami, S., Takahata, R., Kohzaki, D., Kobayashi, S., Hakamazuka, T., and Hozumi, N. (2008). Two of four alternatively spliced isoforms of RUNX2 control osteocalcin gene expression in human osteoblast cells. Gene 413, 817.

Manohar, M., Mooney, A.M., North, J.A., Nakkula, R.J., Picking, J.W., Edon, A., Fishel, R., Poirier, M.G., and Ottesen, J.J. (2009). Acetylation of Histone H3 at the Nucleosome Dyad Alters DNA-Histone Binding. J. Biol. Chem. 284, 23312-23321.

Marfella, C.G.A., and Imbalzano, A.N. (2007). The Chd Family of Chromatin Remodelers. Mutat. Res. 618, 30-40.

Mariman, E.C.M., and Wang, P. (2010). Adipocyte extracellular matrix composition, dynamics and role in obesity. Cell. Mol. Life Sci. 67, 1277-1292.

Marques, M., Laflamme, L., Gervais, A.L., and Gaudreau, L. (2010). Reconciling the positive and negative roles of histone H2A.Z in gene transcription. Epigenetics 5, 267-272. 
Materne, P., Anandhakumar, J., Migeot, V., Soriano, I., Yague-Sanz, C., Hidalgo, E., Mignion, C., Quintales, L., Antequera, F., and Hermand, D. Promoter nucleosome dynamics regulated by signalling through the CTD code. eLife 4.

Mathews, S., Bhonde, R., Gupta, P.K., and Totey, S. (2012). Extracellular matrix protein mediated regulation of the osteoblast differentiation of bone marrow derived human mesenchymal stem cells. Differ. Res. Biol. Divers. 84, 185-192.

Mavrich, T.N., loshikhes, I.P., Venters, B.J., Jiang, C., Tomsho, L.P., Qi, J., Schuster, S.C., Albert, I., and Pugh, B.F. (2008). A barrier nucleosome model for statistical positioning of nucleosomes throughout the yeast genome. Genome Res. 18, 10731083.

McDaniel, I.E., Lee, J.M., Berger, M.S., Hanagami, C.K., and Armstrong, J.A. (2008). Investigations of CHD1 Function in Transcription and Development of Drosophila melanogaster. Genetics 178, 583-587.

McKnight, J.N., Jenkins, K.R., Nodelman, I.M., Escobar, T., and Bowman, G.D. (2011). Extranucleosomal DNA Binding Directs Nucleosome Sliding by Chd1. Mol. Cell. Biol. 31, 4746-4759.

Méndez-Ferrer, S., Michurina, T.V., Ferraro, F., Mazloom, A.R., MacArthur, B.D., Lira, S.A., Scadden, D.T., Ma'ayan, A., Enikolopov, G.N., and Frenette, P.S. (2010). Mesenchymal and haematopoietic stem cells form a unique bone marrow niche. Nature 466, 829-834.

Meneghini, M.D., Wu, M., and Madhani, H.D. (2003). Conserved histone variant H2A.Z protects euchromatin from the ectopic spread of silent heterochromatin. Cell $112,725-736$.

Mills, J.D., Kawahara, Y., and Janitz, M. (2013). Strand-Specific RNA-Seq Provides Greater Resolution of Transcriptome Profiling. Curr. Genomics 14, 173-181.

Min, I.M., Waterfall, J.J., Core, L.J., Munroe, R.J., Schimenti, J., and Lis, J.T. (2011). Regulating RNA polymerase pausing and transcription elongation in embryonic stem cells. Genes Dev. 25, 742-754.

Mito, Y., Henikoff, J.G., and Henikoff, S. (2005). Genome-scale profiling of histone H3.3 replacement patterns. Nat. Genet. 37, 1090-1097.

Mizuta, S., Minami, T., Fujita, H., Kaminaga, C., Matsui, K., Ishino, R., Fujita, A., Oda, K., Kawai, A., Hasegawa, N., et al. (2014). CCAR1/CoCoA pair-mediated recruitment of the Mediator defines a novel pathway for GATA1 function. Genes Cells Devoted Mol. Cell. Mech. 19, 28-51.

Morettini, S., Tribus, M., Zeilner, A., Sebald, J., Campo-Fernandez, B., Scheran, G., Wörle, H., Podhraski, V., Fyodorov, D.V., and Lusser, A. (2011). The chromodomains of CHD1 are critical for enzymatic activity but less important for chromatin localization. Nucleic Acids Res. 39, 3103-3115. 
Mori, T., Sakaue, H., Iguchi, H., Gomi, H., Okada, Y., Takashima, Y., Nakamura, K., Nakamura, T., Yamauchi, T., Kubota, N., et al. (2005). Role of Krüppel-like factor 15 (KLF15) in transcriptional regulation of adipogenesis. J. Biol. Chem. 280, 1286712875.

Mrak, E., Villa, I., Lanzi, R., Losa, M., Guidobono, F., and Rubinacci, A. (2007). Growth hormone stimulates osteoprotegerin expression and secretion in human osteoblast-like cells. J. Endocrinol. 192, 639-645.

Mueller-Planitz, F., Klinker, H., and Becker, P.B. (2013). Nucleosome sliding mechanisms: new twists in a looped history. Nat. Struct. Mol. Biol. 20, 1026-1032.

Mukherjee, R., Jow, L., Croston, G.E., and Paterniti, J.R. (1997). Identification, characterization, and tissue distribution of human peroxisome proliferator-activated receptor (PPAR) isoforms PPARgamma2 versus PPARgamma1 and activation with retinoid $X$ receptor agonists and antagonists. J. Biol. Chem. 272, 8071-8076.

Muse, G.W., Gilchrist, D.A., Nechaev, S., Shah, R., Parker, J.S., Grissom, S.F., Zeitlinger, J., and Adelman, K. (2007). RNA polymerase is poised for activation across the genome. Nat. Genet. 39, 1507-1511.

Naftelberg, S., Schor, I.E., Ast, G., and Kornblihtt, A.R. (2015). Regulation of Alternative Splicing Through Coupling with Transcription and Chromatin Structure. Annu. Rev. Biochem. 84, 165-198.

Nagarajan, S., Benito, E., Fischer, A., and Johnsen, S.A. (2015). H4K12ac is regulated by estrogen receptor-alpha and is associated with BRD4 function and inducible transcription. Oncotarget 6, 7305-7317.

Nelson, J.D., Denisenko, O., and Bomsztyk, K. (2006). Protocol for the fast chromatin immunoprecipitation (ChIP) method. Nat. Protoc. 1, 179-185.

Ng, C.P., Mohamed Sharif, A.R., Heath, D.E., Chow, J.W., Zhang, C.B., Chan-Park, M.B., Hammond, P.T., Chan, J.K., and Griffith, L.G. (2014). Enhanced ex vivo expansion of adult mesenchymal stem cells by fetal mesenchymal stem cell ECM. Biomaterials 35, 4046-4057.

Niyibizi, C., and Eyre, D.R. (1989). Identification of the cartilage alpha $1(\mathrm{XI})$ chain in type $\mathrm{V}$ collagen from bovine bone. FEBS Lett. 242, 314-318.

Oishi, Y., Manabe, I., Tobe, K., Tsushima, K., Shindo, T., Fujiu, K., Nishimura, G., Maemura, K., Yamauchi, T., Kubota, N., et al. (2005). Krüppel-like transcription factor KLF5 is a key regulator of adipocyte differentiation. Cell Metab. 1, 27-39.

Okada, M., Okawa, K., Isobe, T., and Fukagawa, T. (2009). CENP-H-containing Complex Facilitates Centromere Deposition of CENP-A in Cooperation with FACT and CHD1. Mol. Biol. Cell 20, 3986-3995.

Oury, F., Ferron, M., Huizhen, W., Confavreux, C., Xu, L., Lacombe, J., Srinivas, P., Chamouni, A., Lugani, F., Lejeune, H., et al. (2013). Osteocalcin regulates murine and human fertility through a pancreas-bone-testis axis. J. Clin. Invest. 123, 24212433. 
Owen, M., and Friedenstein, A.J. (1988). Stromal stem cells: marrow-derived osteogenic precursors. Ciba Found. Symp. 136, 42-60.

Parekkadan, B., van Poll, D., Suganuma, K., Carter, E.A., Berthiaume, F., Tilles, A.W., and Yarmush, M.L. (2007). Mesenchymal stem cell-derived molecules reverse fulminant hepatic failure. Plos One 2, e941.

Park, D., Shivram, H., and lyer, V.R. (2014). Chd1 co-localizes with early transcription elongation factors independently of H3K36 methylation and releases stalled RNA polymerase II at introns. Epigenetics Chromatin 7, 32.

Patel, A., Chakravarthy, S., Morrone, S., Nodelman, I.M., McKnight, J.N., and Bowman, G.D. (2013). Decoupling nucleosome recognition from DNA binding dramatically alters the properties of the Chd1 chromatin remodeler. Nucleic Acids Res. 41, 1637-1648.

Pavri, R., Zhu, B., Li, G., Trojer, P., Mandal, S., Shilatifard, A., and Reinberg, D. (2006). Histone H2B monoubiquitination functions cooperatively with FACT to regulate elongation by RNA polymerase II. Cell 125, 703-717.

Pérez-Lluch, S., Blanco, E., Tilgner, H., Curado, J., Ruiz-Romero, M., Corominas, M., and Guigó, R. (2015). Absence of canonical marks of active chromatin in developmentally regulated genes. Nat. Genet. 47, 1158-1167.

Persson, J., and Ekwall, K. (2010). Chd1 remodelers maintain open chromatin and regulate the epigenetics of differentiation. Exp. Cell Res. 316, 1316-1323.

Petesch, S.J., and Lis, J.T. (2012). Overcoming the Nucleosome Barrier During Transcript Elongation. Trends Genet. TIG 28, 285-294.

Pihlajaniemi, T., Tamminen, M., Sandberg, M., Hirvonen, H., and Vuorio, E. (1990). The a1 Chain of Type XIII Collagen. Ann. N. Y. Acad. Sci. 580, 440-443.

Pointner, J., Persson, J., Prasad, P., Norman-Axelsson, U., Strålfors, A., Khorosjutina, O., Krietenstein, N., Svensson, J.P., Ekwall, K., and Korber, P. (2012). CHD1 remodelers regulate nucleosome spacing in vitro and align nucleosomal arrays over gene coding regions in S. pombe. EMBO J. 31, 4388-4403.

Polo, J.M., Liu, S., Figueroa, M.E., Kulalert, W., Eminli, S., Tan, K.Y., Apostolou, E., Stadtfeld, M., Li, Y., Shioda, T., et al. (2010). Cell type of origin influences the molecular and functional properties of mouse induced pluripotent stem cells. Nat. Biotechnol. 28, 848-855.

Post, S., Abdallah, B.M., Bentzon, J.F., and Kassem, M. (2008). Demonstration of the presence of independent pre-osteoblastic and pre-adipocytic cell populations in bone marrow-derived mesenchymal stem cells. Bone 43, 32-39.

Pray-Grant, M.G., Daniel, J.A., Schieltz, D., Yates, J.R., and Grant, P.A. (2005). Chd1 chromodomain links histone H3 methylation with SAGA- and SLIK-dependent acetylation. Nature 433, 434-438. 
Quarto, R., Mastrogiacomo, M., Cancedda, R., Kutepov, S.M., Mukhachev, V., Lavroukov, A., Kon, E., and Marcacci, M. (2001). Repair of large bone defects with the use of autologous bone marrow stromal cells. N. Engl. J. Med. 344, 385-386.

Rachner, T.D., Khosla, S., and Hofbauer, L.C. (2011). Osteoporosis: now and the future. Lancet Lond. Engl. 377, 1276-1287.

Radman-Livaja, M., Quan, T.K., Valenzuela, L., Armstrong, J.A., van Welsem, T., Kim, T., Lee, L.J., Buratowski, S., van Leeuwen, F., Rando, O.J., et al. (2012). A Key Role for Chd1 in Histone H3 Dynamics at the 3' Ends of Long Genes in Yeast. PLoS Genet 8, e1002811.

Ramírez, F., Dündar, F., Diehl, S., Grüning, B.A., and Manke, T. (2014). deepTools: a flexible platform for exploring deep-sequencing data. Nucleic Acids Res. gku365.

Ravens, S., Yu, C., Ye, T., Stierle, M., and Tora, L. (2015). Tip60 complex binds to active Pol II promoters and a subset of enhancers and co-regulates the c-Myc network in mouse embryonic stem cells. Epigenetics Chromatin 8, 45.

Ray-Gallet, D., Quivy, J.-P., Scamps, C., Martini, E.M.-D., Lipinski, M., and Almouzni, G. (2002). HIRA Is Critical for a Nucleosome Assembly Pathway Independent of DNA Synthesis. Mol. Cell 9, 1091-1100.

R Development Core Team (2008). R: A Language and Environment for Statistical Computing.

Regard, J.B., Zhong, Z., Williams, B.O., and Yang, Y. (2012). Wnt Signaling in Bone Development and Disease: Making Stronger Bone with Wnts. Cold Spring Harb. Perspect. Biol. 4, a007997.

Reynolds, N., Latos, P., Hynes-Allen, A., Loos, R., Leaford, D., O’Shaughnessy, A., Mosaku, O., Signolet, J., Brennecke, P., Kalkan, T., et al. (2012). NuRD suppresses pluripotency gene expression to promote transcriptional heterogeneity and lineage commitment. Cell Stem Cell 10, 583-594.

Rickard, D.J., Kassem, M., Hefferan, T.E., Sarkar, G., Spelsberg, T.C., and Riggs, B.L. (1996). Isolation and characterization of osteoblast precursor cells from human bone marrow. J. Bone Miner. Res. 11, 312-324.

Robinson, J.T., Thorvaldsdóttir, H., Winckler, W., Guttman, M., Lander, E.S., Getz, G., and Mesirov, J.P. (2011). Integrative Genomics Viewer. Nat. Biotechnol. 29, 2426.

Rodda, S.J., and McMahon, A.P. (2006). Distinct roles for Hedgehog and canonical Wnt signaling in specification, differentiation and maintenance of osteoblast progenitors. Dev. Camb. Engl. 133, 3231-3244.

Rodrigues, L.U., Rider, L., Nieto, C., Romero, L., Karimpour-Fard, A., Loda, M., Lucia, M.S., Wu, M., Shi, L., Cimic, A., et al. (2015). Coordinate loss of MAP3K7 and CHD1 promotes aggressive prostate cancer. Cancer Res. 75, 1021-1034.

Rosen, C.J., and Bouxsein, M.L. (2006). Mechanisms of Disease: is osteoporosis the obesity of bone? Nat. Clin. Pract. Rheumatol. 2, 35-43. 
Rosen, E.D., and MacDougald, O.A. (2006). Adipocyte differentiation from the inside out. Nat. Rev. Mol. Cell Biol. 7, 885-896.

Rosset, P., Deschaseaux, F., and Layrolle, P. (2014). Cell therapy for bone repair. Orthop. Traumatol. Surg. Res. OTSR 100, S107-S112.

Ross-Innes, C.S., Stark, R., Teschendorff, A.E., Holmes, K.A., Ali, H.R., Dunning, M.J., Brown, G.D., Gojis, O., Ellis, I.O., Green, A.R., et al. (2012). Differential oestrogen receptor binding is associated with clinical outcome in breast cancer. Nature 481, 389-393.

Roth, D.B., and Roth, S.Y. (2000). Unequal access: regulating V(D)J recombination through chromatin remodeling. Cell 103, 699-702.

Russell, K.C., Phinney, D.G., Lacey, M.R., Barrilleaux, B.L., Meyertholen, K.E., and O'Connor, K.C. (2010). In Vitro High-Capacity Assay to Quantify the Clonal Heterogeneity in Trilineage Potential of Mesenchymal Stem Cells Reveals a Complex Hierarchy of Lineage Commitment. STEM CELLS 28, 788-798.

Ryan, D.P., Sundaramoorthy, R., Martin, D., Singh, V., and Owen-Hughes, T. (2011). The DNA-binding domain of the Chd1 chromatin-remodelling enzyme contains SANT and SLIDE domains. EMBO J. 30, 2596-2609.

Sabek, O.M., Nishimoto, S.K., Fraga, D., Tejpal, N., Ricordi, C., and Gaber, A.O. (2015). Osteocalcin Effect on Human $\beta$-Cells Mass and Function. Endocrinology 156, 3137-3146.

Saeki, H., and Svejstrup, J.Q. (2009). Stability, Flexibility, and Dynamic Interactions of Colliding RNA Polymerase II Elongation Complexes. Mol. Cell 35, 191-205.

Sainsbury, S., Bernecky, C., and Cramer, P. (2015). Structural basis of transcription initiation by RNA polymerase II. Nat. Rev. Mol. Cell Biol. 16, 129-143.

Salma, N., Xiao, H., Mueller, E., and Imbalzano, A.N. (2004). Temporal Recruitment of Transcription Factors and SWI/SNF Chromatin-Remodeling Enzymes during Adipogenic Induction of the Peroxisome Proliferator-Activated Receptor y Nuclear Hormone Receptor. Mol. Cell. Biol. 24, 4651-4663.

Sarai, N., Nimura, K., Tamura, T., Kanno, T., Patel, M.C., Heightman, T.D., Ura, K., and Ozato, K. (2013). WHSC1 links transcription elongation to HIRA-mediated histone H3.3 deposition. EMBO J. 32, 2392-2406.

Schallmoser, K., Rohde, E., Reinisch, A., Bartmann, C., Thaler, D., Drexler, C., Obenauf, A.C., Lanzer, G., Linkesch, W., and Strunk, D. (2008). Rapid large-scale expansion of functional mesenchymal stem cells from unmanipulated bone marrow without animal serum. Tissue Eng. Part C Methods 14, 185-196.

Schenk, R., Jenke, A., Zilbauer, M., Wirth, S., and Postberg, J. (2011). H3.5 is a novel hominid-specific histone $\mathrm{H} 3$ variant that is specifically expressed in the seminiferous tubules of human testes. Chromosoma 120, 275-285.

Schwartz, S., Meshorer, E., and Ast, G. (2009). Chromatin organization marks exonintron structure. Nat. Struct. Mol. Biol. 16, 990-995. 
Schwartzentruber, J., Korshunov, A., Liu, X.-Y., Jones, D.T.W., Pfaff, E., Jacob, K., Sturm, D., Fontebasso, A.M., Quang, D.-A.K., Tönjes, M., et al. (2012). Driver mutations in histone $\mathrm{H} 3.3$ and chromatin remodelling genes in paediatric glioblastoma. Nature 482, 226-231.

Seligman, M., Eilberg, D.R.G., and Fishman, L. (1975). Mineralization of elastin extracted from human aortic tissues. Calcif. Tissue Res. 17, 229-234.

de la Serna, I.L., Ohkawa, Y., and Imbalzano, A.N. (2006). Chromatin remodelling in mammalian differentiation: lessons from ATP-dependent remodellers. Nat. Rev. Genet. 7, 461-473.

Sethi, A., Jain, A., Zode, G.S., Wordinger, R.J., and Clark, A.F. (2011). Role of TGF $\beta /$ Smad Signaling in Gremlin Induction of Human Trabecular Meshwork Extracellular Matrix Proteins. Invest. Ophthalmol. Vis. Sci. 52, 5251-5259.

Shim, Y.S., Choi, Y., Kang, K., Cho, K., Oh, S., Lee, J., Grewal, S.I.S., and Lee, D. (2012). Hrp3 controls nucleosome positioning to suppress non-coding transcription in eu- and heterochromatin. EMBO J. 31, 4375-4387.

Shin, H., Liu, T., Manrai, A.K., and Liu, X.S. (2009). CEAS: cis-regulatory element annotation system. Bioinforma. Oxf. Engl. 25, 2605-2606.

Siggens, L., Cordeddu, L., Rönnerblad, M., Lennartsson, A., and Ekwall, K. (2015a). Transcription-coupled recruitment of human CHD1 and CHD2 influences chromatin accessibility and histone $\mathrm{H} 3$ and $\mathrm{H} 3.3$ occupancy at active chromatin regions. Epigenetics Chromatin 8.

Simic, R., Lindstrom, D.L., Tran, H.G., Roinick, K.L., Costa, P.J., Johnson, A.D., Hartzog, G.A., and Arndt, K.M. (2003). Chromatin remodeling protein Chd1 interacts with transcription elongation factors and localizes to transcribed genes. EMBO J. 22, 1846-1856.

Simonsen, J.L., Rosada, C., Serakinci, N., Justesen, J., Stenderup, K., Rattan, S.I.S., Jensen, T.G., and Kassem, M. (2002a). Telomerase expression extends the proliferative life-span and maintains the osteogenic potential of human bone marrow stromal cells. Nat. Biotechnol. 20, 592-596.

Sims, R.J., Chen, C.-F., Santos-Rosa, H., Kouzarides, T., Patel, S.S., and Reinberg*, D. (2005). HUMAN BUT NOT YEAST CHD1 BINDS DIRECTLY AND SELECTIVELY TO HISTONE H3 METHYLATED AT LYSINE 4 VIA ITS TANDEM

CHROMODOMAINS. J. Biol. Chem. 280, 41789-41792.

Sims, R.J., Millhouse, S., Chen, C.-F., Lewis, B.A., Erdjument-Bromage, H., Tempst, P., Manley, J.L., and Reinberg, D. (2007). Recognition of trimethylated histone H3 lysine 4 facilitates the recruitment of transcription postinitiation factors and pre-mRNA splicing. Mol. Cell 28, 665-676.

Skene, P.J., Hernandez, A.E., Groudine, M., and Henikoff, S. (2014). The nucleosomal barrier to promoter escape by RNA polymerase II is overcome by the chromatin remodeler Chd1. eLife 3, e02042. 
Smolle, M., Venkatesh, S., Gogol, M.M., Li, H., Zhang, Y., Florens, L., Washburn, M.P., and Workman, J.L. (2012). Chromatin remodelers Isw1 and Chd1 maintain chromatin structure during transcription by preventing histone exchange. Nat. Struct. Mol. Biol. 19, 884-892.

Starcher, B.C., and Urry, D.W. (1973). Elastin coacervate as a matrix for calcification. Biochem. Biophys. Res. Commun. 53, 210-216.

Stasevich, T.J., Hayashi-Takanaka, Y., Sato, Y., Maehara, K., Ohkawa, Y., SakataSogawa, K., Tokunaga, M., Nagase, T., Nozaki, N., McNally, J.G., et al. (2014). Regulation of RNA polymerase II activation by histone acetylation in single living cells. Nature 516, 272-275.

Strahl, B.D., and Allis, C.D. (2000). The language of covalent histone modifications. Nature 403, 41-45.

Struhl, K., and Segal, E. (2013). Determinants of nucleosome positioning. Nat. Struct. Mol. Biol. 20, 267-273.

Subramaniam, M., Jalal, S.M., Rickard, D.J., Harris, S.A., Bolander, M.E., and Spelsberg, T.C. (2002). Further characterization of human fetal osteoblastic hFOB 1.19 and $\mathrm{hFOB} / \mathrm{ER}$ alpha cells: bone formation in vivo and karyotype analysis using multicolor fluorescent in situ hybridization. J. Cell. Biochem. 87, 9-15.

Suto, R.K., Clarkson, M.J., Tremethick, D.J., and Luger, K. (2000). Crystal structure of a nucleosome core particle containing the variant histone H2A.Z. Nat. Struct. Biol. 7, 1121-1124.

Svensson, J.P., Shukla, M., Menendez-Benito, V., Norman-Axelsson, U., Audergon, P., Sinha, I., Tanny, J.C., Allshire, R.C., and Ekwall, K. (2015). A nucleosome turnover map reveals that the stability of histone H4 Lys20 methylation depends on histone recycling in transcribed chromatin. Genome Res. gr.188870.114.

Szenker, E., Lacoste, N., and Almouzni, G. (2012). A developmental requirement for HIRA-dependent H3.3 deposition revealed at gastrulation in Xenopus. Cell Rep. 1, 730-740.

Taipaleenmäki, H., Abdallah, B.M., AIDahmash, A., Säämänen, A.-M., and Kassem, M. (2011). Wnt signalling mediates the cross-talk between bone marrow derived preadipocytic and pre-osteoblastic cell populations. Exp. Cell Res. 317, 745-756.

Takahashi, K., and Yamanaka, S. (2006). Induction of pluripotent stem cells from mouse embryonic and adult fibroblast cultures by defined factors. Cell 126, 663-676.

Tang, Q.-Q., Otto, T.C., and Lane, M.D. (2003). CCAAT/enhancer-binding protein beta is required for mitotic clonal expansion during adipogenesis. Proc. Natl. Acad. Sci. U. S. A. 100, 850-855.

Thakar, A., Gupta, P., Ishibashi, T., Finn, R., Silva-Moreno, B., Uchiyama, S., Fukui, K., Tomschik, M., Ausio, J., and Zlatanova, J. (2009). H2A.Z and H3.3 histone variants affect nucleosome structure: biochemical and biophysical studies. Biochemistry (Mosc.) 48, 10852-10857. 
Tilgner, H., Nikolaou, C., Althammer, S., Sammeth, M., Beato, M., Valcárcel, J., and Guigó, R. (2009). Nucleosome positioning as a determinant of exon recognition. Nat. Struct. Mol. Biol. 16, 996-1001.

Tonna, S., El-Osta, A., Cooper, M.E., and Tikellis, C. (2010). Metabolic memory and diabetic nephropathy: potential role for epigenetic mechanisms. Nat. Rev. Nephrol. 6, 332-341.

Tremethick, D.J. (2007). Higher-Order Structures of Chromatin: The Elusive $30 \mathrm{~nm}$ Fiber. Cell 128, 651-654.

Tropberger, P., Pott, S., Keller, C., Kamieniarz-Gdula, K., Caron, M., Richter, F., Li, G., Mittler, G., Liu, E.T., Bühler, M., et al. (2013). Regulation of transcription through acetylation of H3K122 on the lateral surface of the histone octamer. Cell 152, 859872.

Tsukiyama, T., Palmer, J., Landel, C.C., Shiloach, J., and Wu, C. (1999).

Characterization of the imitation switch subfamily of ATP-dependent chromatinremodeling factors in Saccharomyces cerevisiae. Genes Dev. 13, 686-697.

Twine, N.A., Chen, L., Pang, C.N., Wilkins, M.R., and Kassem, M. (2014a). Identification of differentiation-stage specific markers that define the ex vivo osteoblastic phenotype. Bone 67, 23-32.

Tzaribachev, N., Vaegler, M., Schaefer, J., Reize, P., Rudert, M., Handgretinger, R., and Müller, I. (2008). Mesenchymal stromal cells: a novel treatment option for steroid-induced avascular osteonecrosis. Isr. Med. Assoc. J. IMAJ 10, 232-234.

Undale, A.H., Westendorf, J.J., Yaszemski, M.J., and Khosla, S. (2009).

Mesenchymal Stem Cells for Bone Repair and Metabolic Bone Diseases. Mayo Clin. Proc. 84, 893-902.

Van Holde, K.E., Allen, J.R., Tatchell, K., Weischet, W.O., and Lohr, D. (1980). DNAhistone interactions in nucleosomes. Biophys. J. 32, 271-282.

Venkatesh, S., and Workman, J.L. (2015). Histone exchange, chromatin structure and the regulation of transcription. Nat. Rev. Mol. Cell Biol. 16, 178-189.

Voigt, P., Tee, W.-W., and Reinberg, D. (2013). A double take on bivalent promoters. Genes Dev. 27, 1318-1338.

Voss, T.C., and Hager, G.L. (2014). Dynamic regulation of transcriptional states by chromatin and transcription factors. Nat. Rev. Genet. 15, 69-81.

Waddington, C.H. (1957). The strategy of the genes; a discussion of some aspects of theoretical biology (New York: Macmillan).

Wakitani, S., Nawata, M., Tensho, K., Okabe, T., Machida, H., and Ohgushi, H. (2007). Repair of articular cartilage defects in the patello-femoral joint with autologous bone marrow mesenchymal cell transplantation: three case reports involving nine defects in five knees. J. Tissue Eng. Regen. Med. 1, 74-79. 
Wang, Y., Li, X., and Hu, H. (2014). H3K4me2 reliably defines transcription factor binding regions in different cells. Genomics 103, 222-228.

Weber, C.M., Henikoff, J.G., and Henikoff, S. (2010). H2A.Z nucleosomes enriched over active genes are homotypic. Nat. Struct. Mol. Biol. 17, 1500-1507.

Weber, C.M., Ramachandran, S., and Henikoff, S. (2014). Nucleosomes Are Context-Specific, H2A.Z-Modulated Barriers to RNA Polymerase. Mol. Cell 53, 819830.

Wei, X., Yang, X., Han, Z., Qu, F., Shao, L., and Shi, Y. (2013). Mesenchymal stem cells: a new trend for cell therapy. Acta Pharmacol. Sin. 34, 747-754.

Whitehouse, I., and Tsukiyama, T. (2006). Antagonistic forces that position nucleosomes in vivo. Nat. Struct. Mol. Biol. 13, 633-640.

Wirbelauer, C., Bell, O., and Schübeler, D. (2005). Variant histone H3.3 is deposited at sites of nucleosomal displacement throughout transcribed genes while active histone modifications show a promoter-proximal bias. Genes Dev. 19, 1761-1766.

Xiao, G., Gopalakrishnan, R., Jiang, D., Reith, E., Benson, M.D., and Franceschi, R.T. (2002). Bone morphogenetic proteins, extracellular matrix, and mitogenactivated protein kinase signaling pathways are required for osteoblast-specific gene expression and differentiation in MC3T3-E1 cells. J. Bone Miner. Res. Off. J. Am. Soc. Bone Miner. Res. 17, 101-110.

Xue, Y., Wong, J., Moreno, G.T., Young, M.K., Côté, J., and Wang, W. (1998). NURD, a novel complex with both ATP-dependent chromatin-remodeling and histone deacetylase activities. Mol. Cell 2, 851-861.

Zaher, W., Harkness, L., Jafari, A., and Kassem, M. (2014). An update of human mesenchymal stem cell biology and their clinical uses. Arch. Toxicol. 88, 1069-1082.

Zeitlinger, J., Stark, A., Kellis, M., Hong, J.-W., Nechaev, S., Adelman, K., Levine, M., and Young, R.A. (2007). RNA polymerase stalling at developmental control genes in the Drosophila melanogaster embryo. Nat. Genet. 39, 1512-1516.

Zhang, H., Roberts, D.N., and Cairns, B.R. (2005). Genome-Wide Dynamics of Htz1, a Histone H2A Variant that Poises Repressed/Basal Promoters for Activation through Histone Loss. Cell 123, 219-231.

Zhang, H., Gao, L., Anandhakumar, J., and Gross, D.S. (2014). Uncoupling Transcription from Covalent Histone Modification. PLoS Genet 10, e1004202.

Zhang, Y., Liu, T., Meyer, C.A., Eeckhoute, J., Johnson, D.S., Bernstein, B.E., Nusbaum, C., Myers, R.M., Brown, M., Li, W., et al. (2008). Model-based Analysis of ChIP-Seq (MACS). Genome Biol. 9, R137.

Zhong, Z., Zylstra-Diegel, C.R., Schumacher, C.A., Baker, J.J., Carpenter, A.C., Rao, S., Yao, W., Guan, M., Helms, J.A., Lane, N.E., et al. (2012). Wntless functions in mature osteoblasts to regulate bone mass. Proc. Natl. Acad. Sci. U. S. A. 109, E2197-E2204. 
Zhou, B.O., Yue, R., Murphy, M.M., Peyer, J.G., and Morrison, S.J. (2014). LeptinReceptor-Expressing Mesenchymal Stromal Cells Represent the Main Source of Bone Formed by Adult Bone Marrow. Cell Stem Cell 15, 154-168.

Zhu, Q., and Wani, A.A. (2010). Histone Modifications: Crucial Elements for Damage Response and Chromatin Restoration. J. Cell. Physiol. 223, 283-288.

Zlatanova, J., and Thakar, A. (2008). H2A.Z: View from the Top. Structure 16, 166179. 


\section{Acknowledgements}

I deeply thank my scientific mentor and supervisor Prof. Dr. Steven A. Johnsen. You always motivated me, were supportive and available at all times. I know that you gave honest and encouraging advices. Besides that, I think you are pivotal for the friendly and relaxed working atmosphere in our lab. You have a contagious and inspiring devotion for science which helps to grow in a professional and personal way.

I also thank my thesis committee members Prof. Dr. Heidi Hahn and Prof. Dr. Jürgen Wienands for the great discussions and friendly and helpful advices. Thanks to you I knew who to contact when necessary or in need. In particular, I thank you, Prof. Dr. Heidi Hahn, for your time and for providing the second review of my thesis.

I want to show my gratitude to the department directors Prof. Dr. Matthias Dobbelstein and Prof. Dr. Klaus Pantel by thanking them for the warm welcome to Göttingen and Hamburg, respectively. I am very glad and grateful to have met and worked with Prof. Dr. Hans Will who was so supportive and helpful before and during my thesis. A huge thank you goes to all colleagues from the lab for the open, friendly and funny atmosphere. In particular, I would like to thank Zeynab Najafova, Tareq Hossan, Wanhua Xie and the former members Dr. Upasana Bedi and Dr. Sankari Nagarajan, who offered great advice during the writing of my thesis. I really enjoyed having plenty of interesting discussions, coffee breaks and dinner outings with you and the whole great team.

I am sending big thanks to our collaborators in Denmark, Prof. Dr. Moustapha Kassem and Nicholas Ditzel, who performed the ectopic bone formation experiment.

Finally, I thank those important people in my life that are very special to me. My parents, Christine and Rüdiger Baumgart as well as my brother Markus Baumgart who have given the greatest support, trust and love independent of any circumstances. You are the strongest backing I could ever have wished for.

Insa, last but never least, I especially thank you for being my best friend and soul mate. Your patience, support and innovative concepts move my life into a better direction. 